\title{
One-pot Biocatalytic Cascade Reduction of Cyclic Enimines for the Preparation of Diastereomerically Enriched $N$-Heterocycles
}

Thomas W. Thorpe, ${ }^{\dagger}$ Scott P. France, ${ }^{\dagger,}$ Shahed Hussain, ${ }^{\dagger}$ James R. Marshall, ${ }^{\dagger}$ Wojciech Zawodny, ${ }^{\dagger}$ Juan Mangas-Sanchez, ${ }^{\dagger}$ Sarah L. Montgomery, ${ }^{\dagger}$ Roger M. Howard ${ }^{\ddagger}$ David S. B. Daniels ${ }^{\S}$ Rajesh Kumar, ${ }^{\ddagger}$ Fabio Parmeggiani, ${ }^{\dagger}, *$ Nicholas J. Turner ${ }^{\dagger}, *$

\footnotetext{
$\dagger$ School of Chemistry, University of Manchester, Manchester Institute of Biotechnology, 131 Princess Street, Manchester, M1 7DN (UK).

* Pfizer Worldwide Research and Development, 445 Eastern Point Road, Groton, CT 06340 (USA).

${ }^{\S}$ Pfizer Worldwide Research and Development Discovery Park, Sandwich, Kent, CT13 9NJ (UK).
}

\section{SUPPORTING INFORMATION}




\section{Table of contents}

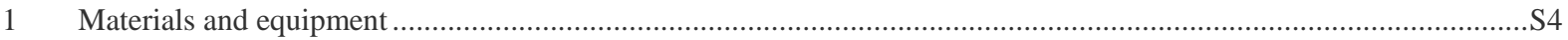

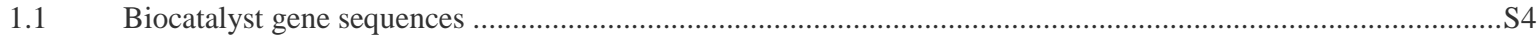

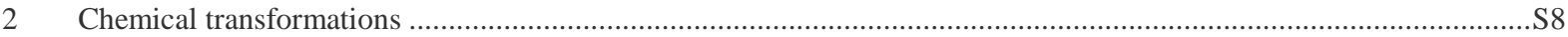

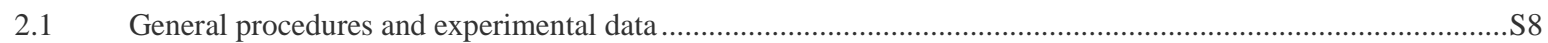

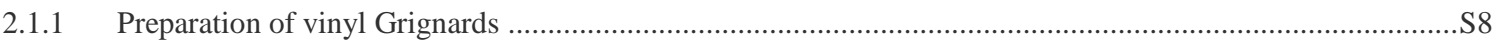

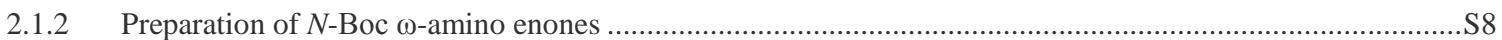

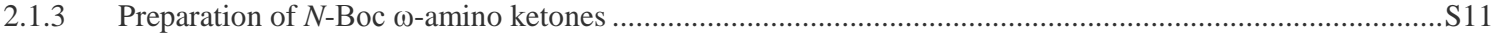

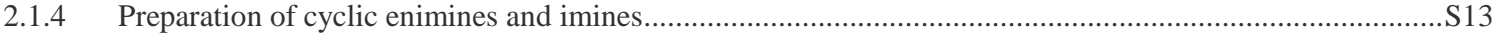

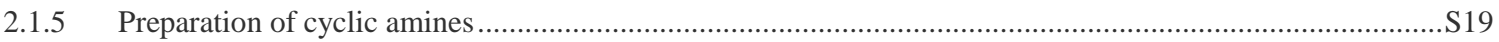

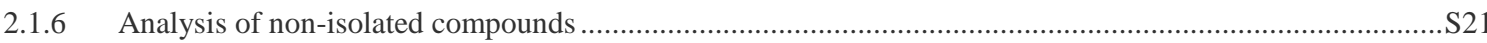

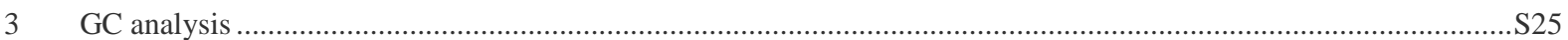

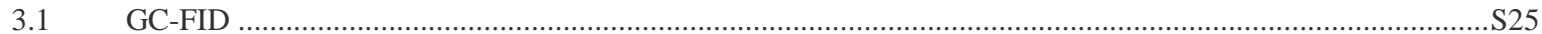

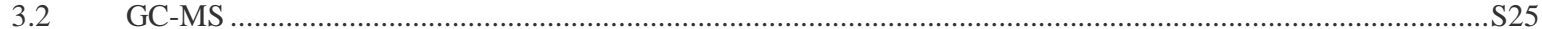

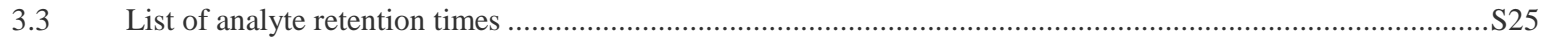

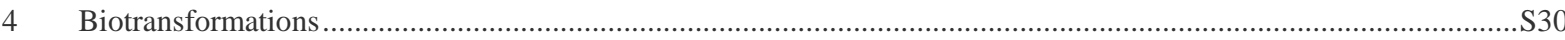

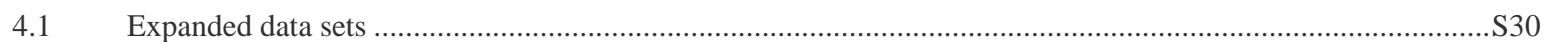

4.1.1 ERED catalysed $\mathrm{C}=\mathrm{C}$ reduction of cyclic enimines (manuscript Table 1) ................................................S30

4.1.2 IRED catalysed reduction of racemic $\alpha$-chiral imine (manuscript Table 2) …..........................................S33

4.1.3 ERED-IRED telescopic synthesis of saturated $N$-heterocycles (manuscript Scheme 3) ............................S34

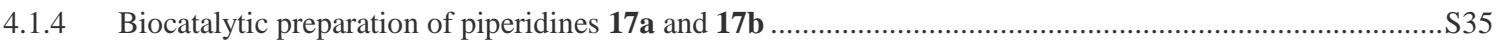

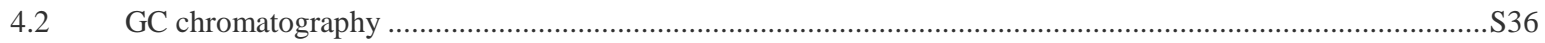

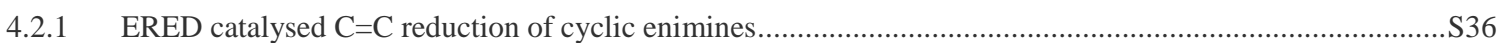

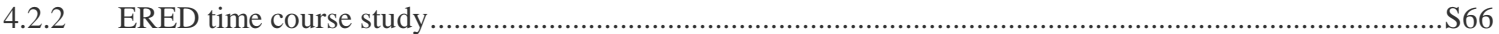

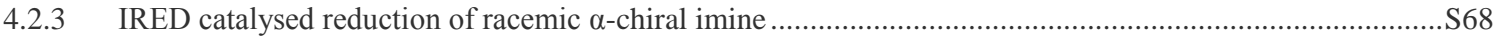

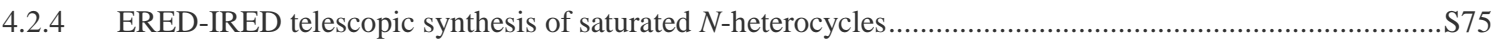




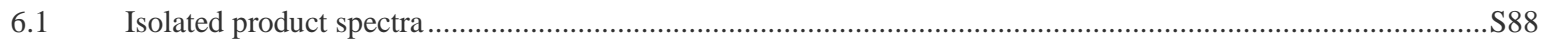

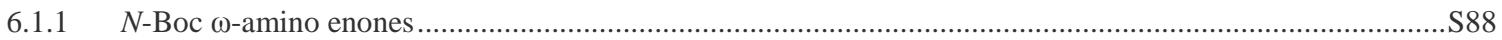

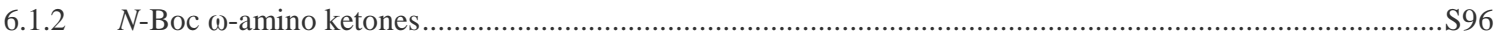

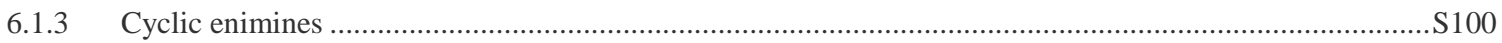

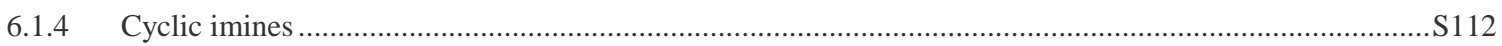

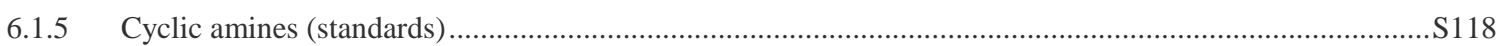

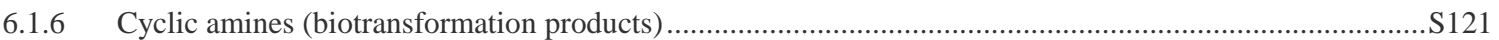

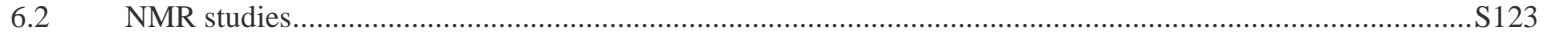

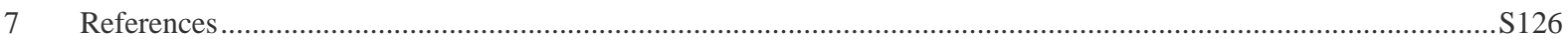




\section{$1 \quad$ Materials and equipment}

Commercially available chemicals were purchased from and Sigma-Aldrich (Poole, Dorset, UK), Alfa Aesar (Karlsruhe, Germany) and Acros Organics (Geel, Belgium) and were used without in-house purification. GC gases were obtained from BOC gases (Guildford, UK).

High-resolution mass spectrometry (HRMS) was recorded using a Waters LCT time-of-flight mass spectrometer, connected to a Waters Alliance LC (Waters, Milford, MA, USA). Waters Masslynx software was used for the data processing.

Small molecule NMR spectroscopy was recorded using a Bruker Advance $\left({ }^{1} \mathrm{H}\right.$ at $400 \mathrm{MHz},{ }^{13} \mathrm{C}$ at $\left.100 \mathrm{MHz}\right)$ and reported chemical shifts $(\delta, \mathrm{ppm})$ are relative to the deuterated solvent residual protic signal.

GC-FID analysis was performed using an Agilent $6850 \mathrm{GC}$ at a constant He flow.

GC-MS analysis was performed using an Agilent 7890B Series GC with 5977B MS-EI detector in positive mode at a constant He flow.

Commercially available biocatalysts were provided as CFEs from Prozomix (PRO-EREDXX), UK and Johnson Mattey (JMENEXXX), UK.

\subsection{Biocatalyst gene sequences}

ERED and IRED were produced according to literature procedures: OYE2, ${ }^{\mathrm{S} 1} \mathrm{OYE} 3,{ }^{\mathrm{S} 1} \mathrm{TOYE},{ }^{\mathrm{S} 2} N t \mathrm{DBR},{ }^{\mathrm{S} 3} \mathrm{PETNR},{ }^{\mathrm{S} 4}(R)-$ IRED,${ }^{\mathrm{S} 5}(S)$-IRED,${ }^{\mathrm{S} 6}$ AdRedAm. ${ }^{\mathrm{S} 7}$ Sequences are provided below.

\begin{tabular}{|l|l|l|l|l|}
\hline Enzyme & Plasmid & Source & Sequence & Ref. \\
\hline OYE2 & pET30a-OYE2 & Saccharomyces cerevisiae & UniProt Q03558 & s1 \\
\hline OYE3 & pET30a-OYE3 & Saccharomyces cerevisiae & UniProt P41816 & s1 \\
\hline TOYE & pET30a-TOYE & Thermoanaerobacter pseudethanolicus & NCBI WP_012268805.1 & s2 \\
\hline NtDBR & pET21b-NtDBR & Nicotiana tabacum & UniProt Q9SLN8 & s3 \\
\hline PETNR & pONR1-H PETNR & Enterobacter cloacae & UniProt P71278 & s4 \\
\hline$(R)$-IRED & pET28a-RIRED & Streptomyces sp. GF3587 & UniProt M4ZRJ3 & s5 \\
\hline$(S)$-IRED & pET28a-SIRED & Streptomyces sp. GF3546 & UniProt M4ZS15 & s6 \\
\hline AdRedAm & pET28a-AdRedAm & Ajellomyces dermatitidis & UniProt C5GTJ9 & s7 \\
\hline
\end{tabular}




\section{OYE2}

ATGCCATTTGTTAAGGACTTTAAGCCACAAGCTTTGGGTGACACCAACTTATTCAAACCA ATCAAAATTGGTAACAATGAACTTCTACACCGTGCTGTCATTCCTCCATTGACTAGAATG AGAGCCCAACATCCAGGTAATATTCCAAACAGAGACTGGGCCGTTGAATACTACGCTCAA CGTGCTCAAAGACCAGGAACCTTGATTATCACTGAAGGTACCTTTCCCTCTCCACAATCT GGGGGTTACGACAATGCTCCAGGTATCTGGTCCGAAGAACAAATTAAAGAATGGACCAAG ATTTTCAAGGCTATTCATGAGAATAAATCGTTCGCATGGGTCCAATTATGGGTTCTAGGT TGGGCTGCTTTCCCAGACACCCTTGCTAGGGATGGTTTGCGTTACGACTCCGCTTCTGAC AACGTGTATATGAATGCAGAACAAGAAGAAAAGGCTAAGAAGGCTAACAACCCACAACAC AGTATAACAAAGGATGAAATTAAGCAATACGTCAAAGAATACGTCCAAGCTGCCAAAAAC TCCATTGCTGCTGGTGCCGATGGTGTTGAAATCCACAGCGCTAACGGTTACTTGTTGAAC CAGTTCTTGGACCCACACTCCAATAACAGAACCGATGAGTATGGTGGATCCATCGAAAAC AGAGCCCGTTTCACCTTGGAAGTGGTTGATGCAGTTGTCGATGCTATTGGCCCTGAAAAA GTCGGTTTGAGATTGTCTCCATATGGTGTCTTCAACAGTATGTCTGGTGGTGCTGAAACC GGTATTGTTGCTCAATATGCTTATGTCTTAGGTGAACTAGAAAGAAGAGCTAAAGCTGGC AAGCGTTTGGCTTTCGTCCATCTAGTTGAACCTCGTGTCACCAACCCATTTTTAACTGAA GGTGAAGGTGAATACAATGGAGGTAGCAACAAATTTGCTTATTCTATCTGGAAGGGCCCA ATTATTAGAGCTGGTAACTTTGCTCTGCACCCAGAAGTTGTCAGAGAAGAGGTGAAGGAT CCTAGAACATTGATCGGTTACGGTAGATTTTTTATCTCTAATCCAGATTTGGTTGATCGT TTGGAAAAAGGGTTACCATTAAACAAATATGACAGAGACACTTTCTACAAAATGTCAGCT GAGGGATACATTGACTACCCTACGTACGAAGAAGCTCTAAAACTCGGTTGGGACAAAAAT TAA

\section{OYE3}

ATGCCATTTGTAAAAGGTTTTGAGCCGATCTCCCTAAGAGACACAAACCTTTTTGAACCA ATTAAGATTGGTAACACTCAGCTTGCACATCGTGCGGTTATGCCCCCATTGACCAGAATG AGGGCCACTCACCCCGGAAATATTCCAAATAAGGAGTGGGCTGCTGTGTATTATGGTCAG CGTGCTCAAAGACCTGGTACCATGATCATCACGGAAGGTACGTTTATTTCCCCTCAAGCC GGCGGCTATGACAACGCCCCTGGGATTTGGTCTGATGAGCAGGTCGCTGAGTGGAAGAAT ATCTTTTTAGCCATCCATGATTGTCAGTCGTTCGCGTGGGTACAACTTTGGTCTTTAGGC TGGGCATCCTTCCCAGACGTATTGGCAAGAGACGGGTTACGCTATGACTGTGCATCTGAC AGAGTGTATATGAATGCTACGTTACAAGAAAAGGCCAAAGATGCGAATAATCTCGAACAT AGTTTGACTAAAGACGACATTAAACAGTATATCAAGGATTACATCCATGCGGCTAAGAAT TCTATCGCGGCTGGCGCCGATGGTGTAGAAATTCATAGCGCCAATGGGTACTTGTTGAAT CAGTTCTTGGATCCACATTCTAATAAGAGGACCGACGAATACGGCGGAACGATCGAAAAC AGGGCCCGCTTTACACTGGAGGTTGTCGATGCTCTTATCGAAACTATCGGTCCTGAACGG GTGGGTTTGAGGTTGTCGCCGTACGGCACTTTTAACAGTATGTCTGGGGGTGCTGAACCA GGTATTATCGCTCAATATTCGTATGTTTTGGGTGAATTAGAGAAGAGGGCAAAGGCTGGT AAGCGTTTGGCCTTTGTGCACCTCGTTGAACCACGTGTCACGGACCCATCGTTGGTGGAG GGCGAAGGAGAATATTCCGAGGGTACTAACGATTTTGCCTACTCTATATGGAAGGGTCCA ATCATCAGAGCTGGTAATTACGCTCTTCATCCAGAAGTGGTTAGAGAACAAGTAAAGGAT CCCAGAACCTTGATAGGCTATGGTAGATTCTTCATCTCTAACCCAGATTTAGTCTACCGT TTAGAAGAGGGCCTGCCATTGAACAAGTATGACAGAAGTACCTTCTACACCATGTCCGCG GAAGGTTATACCGACTACCCAACATATGAAGAGGCAGTAGATTTAGGTTGGAACAAGAAC TGA

\section{TOYE}

ATGAGTATTTTACATATGCCTTTAAAGATAAAGGATATTACAATAAAAAATAGAATCATG ATGTCTCCTATGTGTATGTACTCAGCTTCTACAGATGGGATGCCAAATGACTGGCATATA GTTCATTACGCCACAAGGGCTATTGGTGGAGTAGGACTTATTATGCAAGAAGCCACAGCT GTTGAGAGCAGAGGAAGAATAACTGATCATGACCTTGGCATATGGAATGATGAACAAGTT AAAGAATTAAAAAAAATTGTAGACATTTGTAAAGCAAATGGCGCTGTGATGGGAATACAG CTTGCTCATGCAGGAAGAAAATGTAATATATCCTACGAGGATGTCGTAGGACCTTCCCCT ATCAAAGCAGGAGACCGCTACAAACTTCCAAGAGAATTATCAGTTGAGGAAATAAAATCT ATAGTAAAAGCTTTTGGGGAAGCTGCTAAAAGGGCTAACTTAGCAGGTTATGATGTAGTT GAAATACATGCAGCTCACGGCTATTTAATCCACGAATTTCTTTCTCCTCTTTCAAATAAA CGAAAAGATGAATACGGCAATAGCATTGAAAATAGAGCAAGATTTTTAATTGAAGTGATA GATGAAGTTAGAAAAAATTGGCCTGAAAATAAACCTATTTTCGTGCGGGTATCTGCAGAT GATTACATGGAAGGCGGAATAAACATAGATATGATGGTAGAATATATCAACATGATAAAA GACAAAGTTGATTTAATTGATGTAAGCAGTGGAGGACTTTTAAATGTTGATATAAATCTA TATCCTGGATATCAAGTTAAATACGCTGAAACAATTAAAAAGCGCTGTAATATAAAAACT TCTGCGGTAGGATTAATAACGACACAAGAGCTTGCAGAAGAAATTCTTTCAAATGAAAGG GCAGACTTAGTTGCACTTGGAAGAGAACTTTTAAGAAATCCCTATTGGGTTCTGCATACC TACACTTCAAAGGAAGACTGGCCAAAACAATATGAAAGAGCTTTTAAAAAATAA 


\section{$N t \mathrm{DBR}$}

ATGGCAGAAGAAGTGAGCAACAAACAGGTCATTCTTAAAAACTATGTCACAGGTTACCCT AAGGAATCCGACATGGAAATCAAGAATGTCACCATTAAACTCAAAGTTCCAGAAGGTTCT AATGATGTGGTTGTGAAGAATCTTTACTTGTCTTGTGACCCTTATATGCGTAGCCGCATG AGGAAAATTGAGGGTAGCTATGTTGAATCCTTCGCTCCTGGCTCCCCTATCACGGGATAT GGAGTGGCTAAAGTTTTGGAGTCTGGTGATCCAAAATTCCAAAAAGGTGACTTAGTTTGG GGAATGACTGGATGGGAAGAGTATAGTATTATAACACCTACTCAGACTCTCTTTAAAATT CATGACAAGGATGTGCCTCTTTCCTACTACACAGGAATCCTCGGGATGCCTGGGATGACA GCTTATGCTGGTTTTCATGAGGTTTGCTCCCCCAAGAAGGGGGAAACTGTCTTTGTTTCA GCTGCATCTGGAGCAGTTGGTCAGCTCGTTGGGCAATTTGCAAAGATGCTGGGTTGCTAC GTTGTTGGTAGTGCTGGAAGCAAAGAAAAGGTTGATCTGTTGAAGAGCAAATTTGGGTTT GACGAAGCTTTTAACTATAAAGAGGAGCAGGATTTAAGTGCAGCTTTGAAGAGGTACTTC CCTGATGGAATTGACATCTACTTTGAGAATGTGGGAGGGAAGATGCTTGATGCAGTTCTT GTGAACATGAAACTCTATGGCCGTATTGCTGTGTGTGGGATGATTTCGCAATACAACCTT GAGCAGACTGAAGGAGTGCACAACTTGTTTTGCCTCATCACAAAACGAATCCGCATGGAA GGATTTCTTGTTTTTGATTACTATCATCTTTACCCCAAATATTTGGAAATGGTCATTCCT CAAATAAAGGCAGGCAAGGTTGTTTATGTGGAAGATGTTGCCCATGGCCTTGAAAGTGCT CCCACTGCTCTAGTTGGTCTCTTCTCTGGTCGCAATATTGGAAAGCAAGTCGTGATGGTT TCGCGTGAATGA

\section{PETNR}

ATGTCCGCTGAAAAGCTGTTTACCCCACTGAAAGTGGGTGCCGTTACTGCCCCAAACCGC GTGTTTATGGCCCCACTTACCCGTCTGCGCAGCATCGAGCCGGGCGATATCCCAACGCCA TTGATGGGTGAGTATTACCGCCAGCGCGCCAGCGCGGGCCTGATTATCTCCGAAGCCACG CAGATTTCTGCTCAGGCAAAAGGCTACGCCGGTGCACCGGGTCTGCACAGCCCGGAACAG ATCGCCGCGTGGAAAAAAATCACCGCAGGCGTGCATGCTGAAGATGGCCGTATTGCGGTT CAGCTGTGGCACACCGGTCGTATCTCACACAGCAGCATCCAGCCTGGCGGTCAGGCGCCG GTTTCTGCCTCTGCCCTGAACGCCAATACCCGCACTTCCCTGCGCGATGAAAACGGTAAT GCGATCCGCGTCGACACCACCACGCCACGCGCGCTGGAGCTGGACGAGATCCCGGGTATC GTGAATGATTTCCGTCAGGCCGTCGCCAACGCCCGGGAAGCGGGCTTCGACCTGGTTGAG CTTCACTCTGCGCACGGTTACCTGCTGCATCAGTTCCTGTCCCCGTCTTCCAACCAGCGT ACCGACCAGTACGGCGGCAGCGTTGAAAACCGCGCGCGTCTGGTGCTTGAAGTGGTGGAT GCTGTCTGTAATGAGTGGAGCGCAGACCGCATTGGTATTCGTGTCTCCCCGATCGGTACT TTCCAGAACGTCGACAACGGTCCGAACGAAGAAGCAGACGCGCTGTATCTGATTGAAGAG CTGGCGAAACGCGGTATCGCCTATCTGCACATGTCCGAGACGGACTTGGCAGGCGGCAAG CCTTACAGTGAAGCCTTCCGTCAGAAAGTGCGCGAGCGCTTCCACGGCGTGATTATCGGG GCGGGTGCGTATACGGCAGAAAAAGCCGAGGATTTGATCGGTAAAGGCCTGATCGACGCC GTGGCCTTTGGCCGTGACTACATTGCTAACCCGGATCTGGTTGCCCGTTTGCAGAAAAAA GCCGAACTGAACCCGCAGCGTCCTGAAAGCTTCTATGGCGGCGGCGCGGAAGGTTATACC GACTACCCTTCACTGTAA

\section{$(R)$-IRED}

ATGGGCGACAACCGCACCCCGGTGACGGTCATCGGCCTCGGCCTCATGGGACAGGCGCTC GCCGCCGCGTTCCTCGAGGCCGGCCACACCACGACCGTGTGGAACCGTTCGGCGGGCAAG GCCGAGCAGCTCGTCTCGCAGGGCGCGGTGCAGGCCGCCACGCCCGCCGACGCGGTGGCC GCCAGTGAGCTGGTCGTGGTCTGCCTGTCGACGTACGACAACATGCACGACGTGATCGGT TCGCTCGGTGAGTCCCTCCGGGGCAAGGTCATCGTGAACCTGACGTCCGGCTCGTCGGAC CAGGGCCGCGAGACGGCGGCGTGGGCCGAGAAGCAGGGCGTGGAGTACCTCGACGGCGCG ATCATGATCACGCCGCCCGGCATCGGCACCGAGACCGCCGTCCTCTTCTACGCCGGTACG CAGAGCGTGTTCGAGAAGTACGAGCCCGCGCTCAAGCTGCTCGGCGGCGGCACCACGTAC CTCGGCACCGACCACGGTATGCCCGCGCTGTACGACGTGTCGCTGCTCGGCCTCATGTGG GGCACGCTCAACAGCTTCCTGCACGGTGTCGCCGTGGTGGAGACCGCGGGCGTCGGCGCC CAGCAGTTCCTGCCGTGGGCGCACATGTGGCTGGAGGCGATCAAGATGTTCACCGCCGAC TACGCGGCCCAGATCGACGCCGGTGACGGCAAGTTCCCCGCCAACGACGCCACGCTGGAG ACGCACCTGGCGGCGCTCAAGCACCTGGTGCACGAGAGCGAGGCGCTGGGTATCGACGCC GAACTGCCGAAGTACTCCGAGGCGTTGATGGAGCGCGTCATCAGCCAGGGTCACGCCAAG AACAGCTACGCGGCCGTGCTCAAGGCGTTCCGCAAGCCGTCGGAGTGA 


\section{(S)-IRED}

ATGAGCAAACAGTCAGTTACGGTGATTGGTCTGGGTCCGATGGGTCAAGCGATGGTCAAT ACCTTTCTGGATAATGGTCACGAAGTGACCGTGTGGAACCGTACGGCGTCAAAAGCAGAA GCTCTGGTGGCGCGCGGCGCAGTTCTGGCACCGACCGTCGAAGATGCTCTGAGCGCGAAT GAACTGATTGTTCTGTCTCTGACCGATTATGACGCCGTGTACGCAATCCTGGAACCGGTT ACGGGCTCACTGTCGGGTAAAGTGATTGCAAACCTGAGCTCTGATACCCCGGACAAAGCG CGTGAAGCGGCCAAATGGGCAGCTAAACATGGTGCGAAACATCTGACCGGCGGTGTGCAG GTTCCGCCGCCGCTGATCGGCAAACCGGAAAGTTCCACCTATTACTCCGGTCCGAAAGAT GTTTTTGACGCCCATGAAGATACCCTGAAAGTCCTGACGAACGCCGATTATCGTGGTGAA GATGCAGGTCTGGCCGCAATGTATTACCAGGCGCAAATGACCATTTTCTGGACCACGATG CTGAGCTATTACCAGACGCTGGCTCTGGGCCAAGCGAATGGTGTTAGTGCTAAAGAACTG CTGCCGTATGCCACCATGATGACGTCCATGATGCCGCATTTTCTGGAACTGTATGCTCAG CACGTCGATTCTGCGGACTATCCGGGTGATGTGGACCGTCTGGCGATGGGCGCAGCTTCA GTCGATCACGTGCTGCATACCCACCAAGATGCGGGTGTTAGCACCGTCCTGCCGGCCGCA GTGGCCGAAATCTTCAAAGCCGGTATGGAAAAAGGCTTTGCTGAAAATTCGTTCTCCTCT CTGATTGAAGTCCTGAAAAAACCGGCAGTGTAA

\section{$A d \operatorname{RedAm}$}

ATGGCAAACTCACCGGTATCCGTCTTCGGCCTTGGAGCCATGGGCACCGCCCTGGCCACC CAATTCCTTCGAAAAGGCCACAAAACCACAGTCTGGAACAGGACCCCGGCCAAAGCACAA CCCCTTATCGCTATCGGCGCATCCCATGCTCCCACAATCGACTCTGCCGCCGCGGCTAGC TCTTTACTCATAATCTGCCAACTGGATAAAGCCTCCGTCATGCAAACCCTCCAACAAGCC CCAACGGCATGGGCCGCAAAGACAATTGTTGACCTTACAAACGGCACACCAGCCCACGCC AGAGAGACGGCGGACTGGGCTCTCGCGCACGGGGCTCGATACATCCACGGCGGAATCATG GCCGTACCATTCATGATCGGCCAGCCTGATGCCATGATCCTCTACAGCGGACCAGCAGAG GTGTTCGAGGGGGTAAAGGATACCCTCTCCGTGCTGGGCACCAACACATACGTCGGCGAG GACGTGGGGCTTGCCTCGCTGCACGACCTGGCTCTTCTGAGTGGCATGTACGGCCTGTTC AGCGGCTTCACGCACGCCGTGGCGTTGGTGCAGTCCGCCAACATTCCTGCGGCAGGATTT GTTGCGACGCAGCTCATCCCCTGGCTGACTGCGATGACGCAGCATTTGAATCTGTTGGCG ACGCAGGTGGATGAGAAGGATTACGGGGATGGCGGGTCGAGTTTGGACATGCAGGCGAAG GCTGCGCCAAATATATTGGAGGCGAGTCAAGCCCAGGGTGTTTCGGTGGAACTTATTCAG CCTATTTTCAAGTTGATTGAGAGGAGGGTTGAGGAAGGCAAGGGTAGTGAGGGGCTTGCT GCGCTTGTTGGGATGATCATGAAAGGGGGCACAAAGGACAGTGTGTGA 


\subsection{General procedures and experimental data}

Scheme S1. Synthesis of cyclic enimine substrates 4-6a-d and of analytical reference standards 13-18a-d.

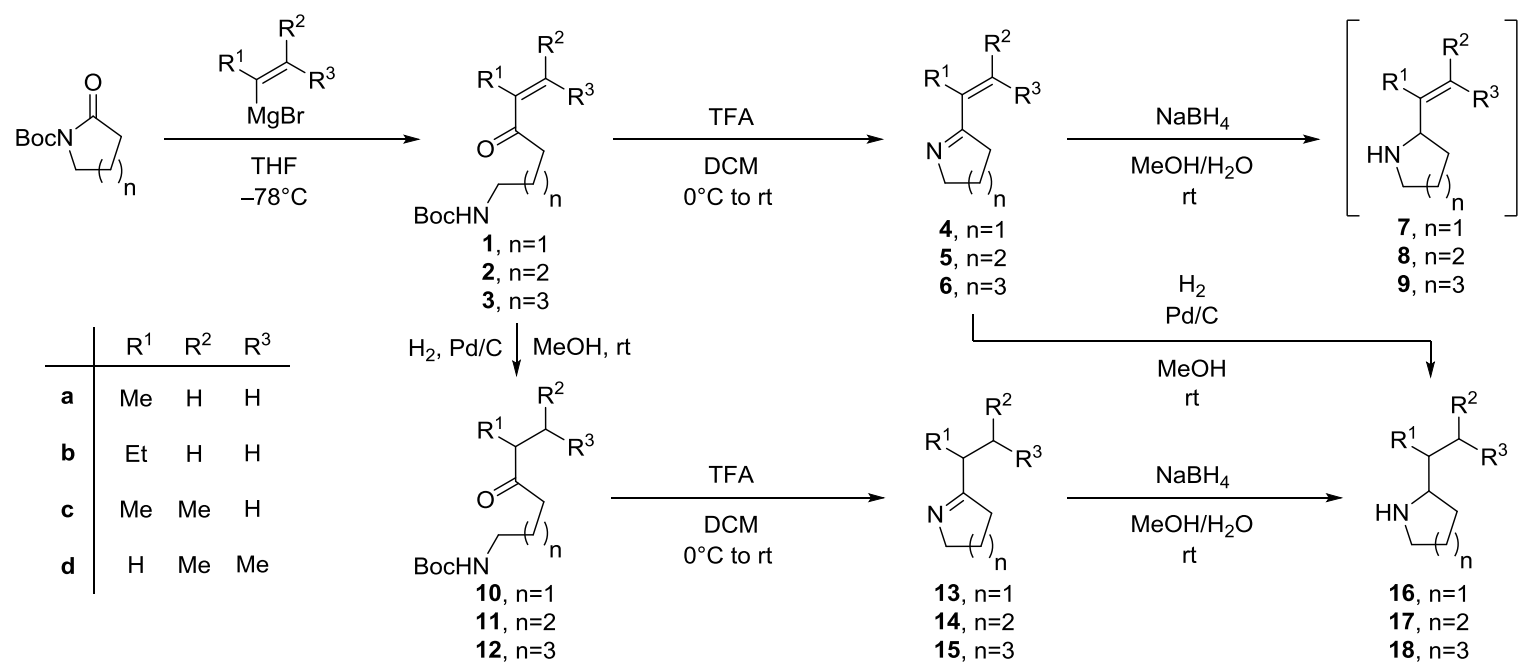

Cyclic enimines were prepared through the vinyl Grignard ring opening of $N$-Boc lactams using the previously published method. ${ }^{\mathrm{S} 8}$ In some instances the $N$-Boc protected amino enones could not be isolated and a mix of di- $N$-Boc protected amino enones was taken directly into the deprotection step. Pd catalysed reduction of $N$-Boc protected amino enones followed by TFA deprotection allowed for the preparation of cyclic imines which could then be further reduced to the final $N$ heterocycles.

\subsubsection{Preparation of vinyl Grignards}

Mg turnings (1.5 eq) were stirred for 15 mins in a two-necked round bottomed flask fitted with a condenser, under a blanket of nitrogen before the addition of dry THF $\left(1.1 \mathrm{~mL} \cdot \mathrm{mmol}^{-1}\right)$ and catalytic $\mathrm{I}_{2}$. Subsequently vinyl bromides $(1 \mathrm{eq})$ were added dropwise to the suspension which initiated a self-propagating reaction which was left for 15 mins. The vinyl Grignards were allowed to cool before being taken through into the next step without characterisation or further purification.

\subsubsection{Preparation of $N$-Boc $\omega$-amino enones}

The corresponding vinyl Grignard $(1.1-2 \mathrm{eq})$ was added dropwise to a cooled $\left(-78^{\circ} \mathrm{C}\right)$ solution of $N$-Boc lactams $(1 \mathrm{eq}, 0.3$ $\mathrm{M})$ in dry THF causing the solution to become cloudy. The reaction was stirred at $-78{ }^{\circ} \mathrm{C}$ overnight, then quenched with $\mathrm{NH}_{4} \mathrm{Cl}$ (sat. aq), before being diluted with $\mathrm{H}_{2} \mathrm{O}$ and allowed to warm to room temperature. The suspension was extracted into DCM (3 x $20 \mathrm{~mL})$, dried with magnesium sulfate and concentrated in vacuo to give a crude oil which was then purified over silica gel to afford the desired product or a mix of mono- and di- $N$-Boc amino enones which were then taken directly into the next step.

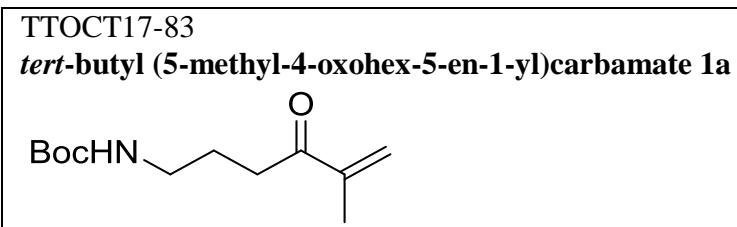

$\mathrm{N}$-Boc-butyrolactam (950 mg, $5 \mathrm{mmol}$ ) was reacted with isopropenylmagnesium bromide (1 M solution in THF, $5 \mathrm{~mL}, 5$ mmol). Purification over silica gel (20\% v/v ethyl acetate-cyclohexane) gave the product ( $400 \mathrm{mg}, 36 \%)$ as a light yellow solid.

${ }^{1} \mathbf{H}$ NMR $\left(400 \mathrm{MHz}, \mathrm{CDCl}_{3}\right) \delta 5.96(\mathrm{~s}, 1 \mathrm{H}), 5.77(\mathrm{~s}, 1 \mathrm{H}), 4.59(\mathrm{~s}, 1 \mathrm{H}), 3.15(\mathrm{q}, J=6.6 \mathrm{~Hz}, 2 \mathrm{H}), 2.73(\mathrm{t}, J=7.2 \mathrm{~Hz}, 2 \mathrm{H}), 1.87$ $(\mathrm{t}, J=1.1 \mathrm{~Hz}, 3 \mathrm{H}), 1.81(\mathrm{p}, J=7.0 \mathrm{~Hz}, 2 \mathrm{H}), 1.43(\mathrm{~s}, 9 \mathrm{H})$.

${ }^{13} \mathrm{C}$ NMR $\left(100 \mathrm{MHz}, \mathrm{CDCl}_{3}\right) \delta 201.7,156.2,144.6,124.8,79.3,77.5,77.2,76.8,40.3,34.7,28.5,24.9,17.8$.

MS (ESI) $\mathrm{m} / \mathrm{z}=250.1\left[\mathrm{M}+\mathrm{Na}^{+}\right]$ 


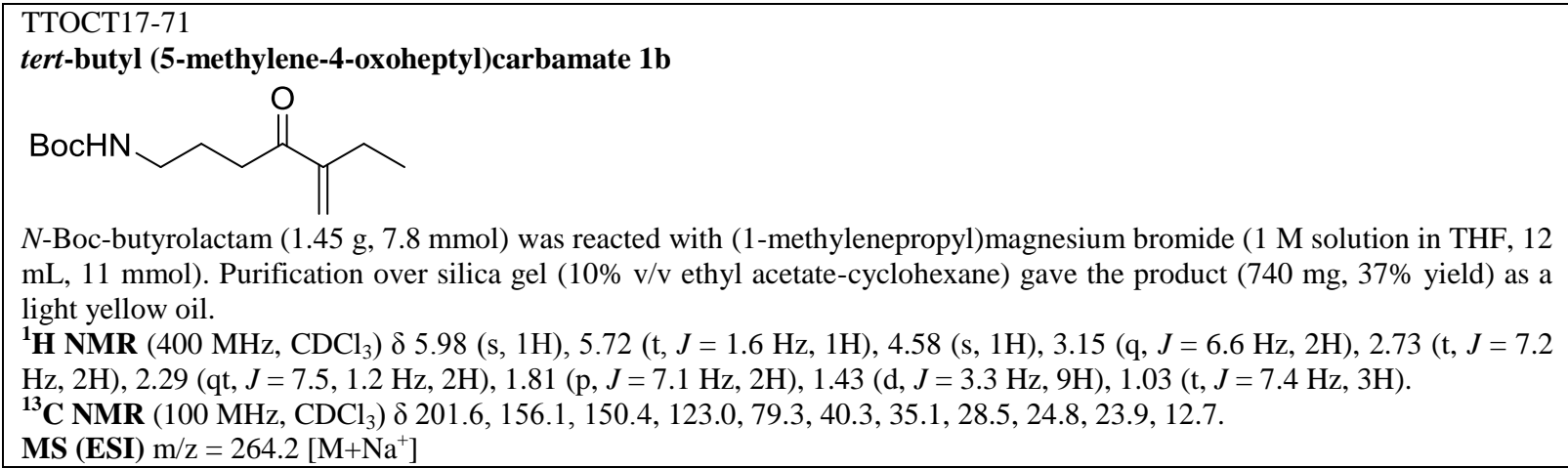

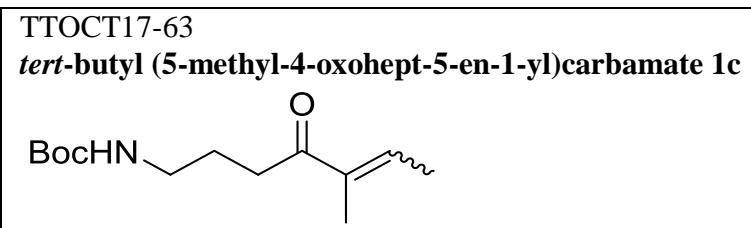

$N$-Boc-butyrolactam $(1 \mathrm{~g}, 5.2 \mathrm{mmol})$ was reacted with (2-methyl-1-propenyl)magnesium bromide $(0.5 \mathrm{M}$ solution in THF, $13 \mathrm{~mL}, 6 \mathrm{mmol})$. Purification over silica gel $(10 \% \mathrm{v} / \mathrm{v}$ ethyl acetate-cyclohexane) gave the yellow oil product as an inseparable mixture of the corresponding geometric isomers (200 mg, 74:26 E:Z, 17\%).

$\mathrm{CCHCH}_{3}, E$-isomer

${ }^{1} \mathbf{H}$ NMR $\left(400 \mathrm{MHz}, \mathrm{CDCl}_{3}\right) \delta 6.83-6.64\left(\mathrm{~m}, 1 \mathrm{H}, \mathrm{CCHCH}_{3}, E\right.$-isomer), 5.81 (qd, J = 6.5, 5.8, 3.0 Hz, 1H, $\mathrm{CCHCH}_{3}, \mathrm{Z}-$ isomer), $4.60(\mathrm{~s}, 1 \mathrm{H}), 3.15(\mathrm{q}, \mathrm{J}=6.5 \mathrm{~Hz}, 2 \mathrm{H}), 2.69(\mathrm{t}, \mathrm{J}=7.2 \mathrm{~Hz}, 1 \mathrm{H}), 2.57(\mathrm{t}, \mathrm{J}=7.1 \mathrm{~Hz}, 1 \mathrm{H}), 1.91(\mathrm{p}, \mathrm{J}=1.6 \mathrm{~Hz}, 2 \mathrm{H})$, $1.87-1.75(\mathrm{~m}, 6 \mathrm{H}), 1.56(\mathrm{~s}, 1 \mathrm{H}), 1.43(\mathrm{~s}, 9 \mathrm{H})$.

MS (ESI) $\mathrm{m} / \mathrm{z}=264.2\left[\mathrm{M}+\mathrm{Na}^{+}\right]$

\section{TTOCT17-12}

tert-butyl (6-methyl-4-oxohept-5-en-1-yl)carbamate 1d

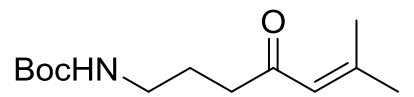

$N$-Boc-butyrolactam (1 g, $5 \mathrm{mmol}$ ) was reacted with (2-methyl-1-propenyl)magnesium bromide $(0.5 \mathrm{M}$ solution in THF, 12 $\mathrm{mL}, 5.9 \mathrm{mmol})$. Purification over silica gel $(10 \% \mathrm{v} / \mathrm{v}$ ethyl acetate-cyclohexane) gave the yellow oil product as an inseparable mixture of the corresponding di- and mono-N-Boc amino enones $(550 \mathrm{mg})$.

MS (ESI) $\mathrm{m} / \mathrm{z}=264.2\left[\mathrm{M}+\mathrm{Na}^{+}\right]$

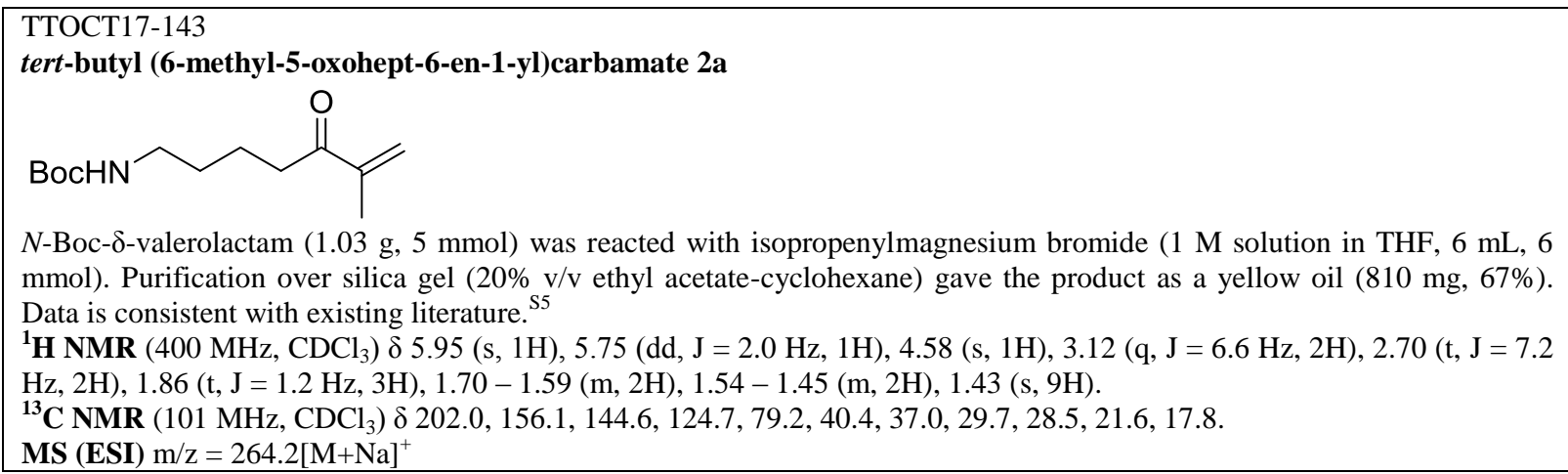




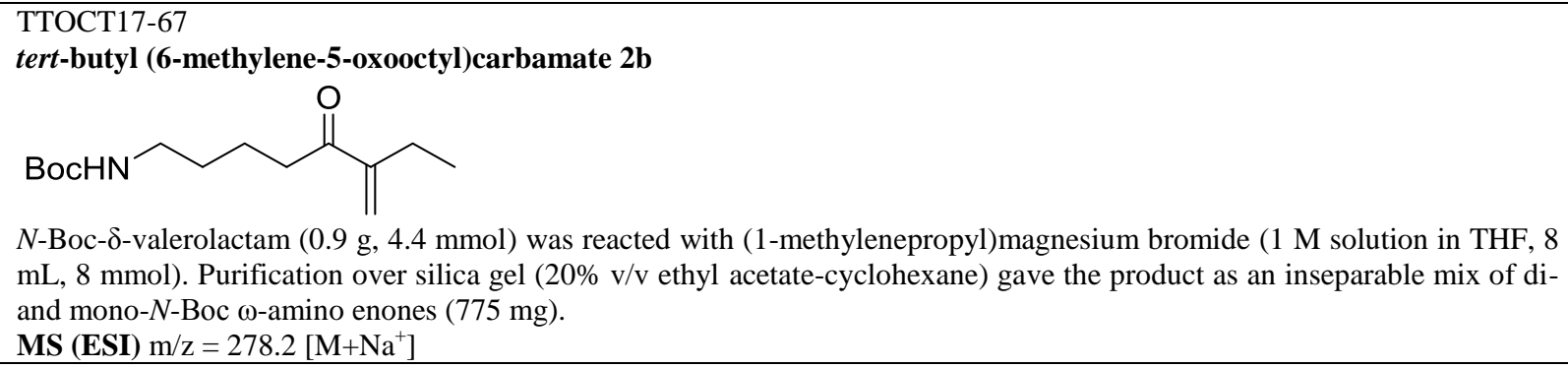

$N$-Boc- $\delta$-valerolactam $(0.9 \mathrm{~g}, 4.4 \mathrm{mmol})$ was reacted with (1-methylenepropyl)magnesium bromide (1 M solution in THF, 8 $\mathrm{mL}, 8 \mathrm{mmol})$. Purification over silica gel ( $20 \% \mathrm{v} / \mathrm{v}$ ethyl acetate-cyclohexane) gave the product as an inseparable mix of diand mono- $N$-Boc $\omega$-amino enones $(775 \mathrm{mg}$ ).

\section{TTOCT17-58}

tert-butyl (6-methyl-5-oxooct-6-en-1-yl)carbamate 2c<smiles>CC=C(C)C(=O)CCCCNC(=O)O</smiles>

$N$-Boc- $\delta$-valerolactam $(1 \mathrm{~g}, 4.9 \mathrm{mmol})$ was reacted with (but-2-en-2-yl)magnesium bromide $(0.5 \mathrm{M}$ solution in THF, $13 \mathrm{~mL}$, $6.4 \mathrm{mmol})$. Purification over silica gel $(20 \% \mathrm{v} / \mathrm{v}$ ethyl acetate-cyclohexane) gave the product and an inseparable mixture of the corresponding isomers $(200 \mathrm{mg}, 43: 57 \mathrm{E}: \mathrm{Z})$.

${ }^{1} \mathbf{H}$ NMR $\left(400 \mathrm{MHz}, \mathrm{CDCl}_{3}\right) \delta 6.74\left(\mathrm{qd}, \mathrm{J}=6.9,1.5 \mathrm{~Hz}, 1 \mathrm{H}, \mathrm{CCHCH}_{3}, E\right.$-isomer), 5.78 (dddd, $\mathrm{J}=8.8,7.2,5.7,1.5 \mathrm{~Hz}, 1 \mathrm{H}$, $\mathrm{CCHCH}_{3} \mathrm{Z}$-isomer), $4.57(\mathrm{~s}, 1 \mathrm{H}), 3.12(\mathrm{~d}, \mathrm{~J}=6.7 \mathrm{~Hz}, 2 \mathrm{H}), 2.66(\mathrm{t}, \mathrm{J}=7.3 \mathrm{~Hz}, 1 \mathrm{H}), 2.54(\mathrm{t}, \mathrm{J}=7.1 \mathrm{~Hz}, 1 \mathrm{H} \mathrm{COCH} E-$ isomer), $1.90\left(\mathrm{p}, \mathrm{J}=1.6 \mathrm{~Hz}, 1 \mathrm{H}, \mathrm{m}, 3 \mathrm{H}, \mathrm{CCH}_{3}, Z\right.$-isomer), $1.86(\mathrm{~d}, \mathrm{~J}=1.2 \mathrm{~Hz}, 1 \mathrm{H}), 1.84(\mathrm{~d}, \mathrm{~J}=1.4 \mathrm{~Hz}, 1 \mathrm{H}), 1.82(\mathrm{q}, \mathrm{J}=1.6$ $\mathrm{Hz}, 1 \mathrm{H}), 1.77(\mathrm{t}, \mathrm{J}=1.3 \mathrm{~Hz}, 2 \mathrm{H}), 1.70-1.46(\mathrm{~m}, 5 \mathrm{H}), 1.44(\mathrm{~s}, 8 \mathrm{H})$.

MS (ESI) $\mathrm{m} / \mathrm{z}=278.2\left[\mathrm{M}+\mathrm{Na}^{+}\right]$

\section{TTOCT17-27}

tert-butyl (7-methyl-5-oxooct-6-en-1-yl)carbamate 2d<smiles>CC(C)=CC(=O)CCCCNC(=O)O</smiles>

$N$-Boc- $\delta$-valerolactam $(1 \mathrm{~g}, 5 \mathrm{mmol})$ was reacted with (2-methyl-1-propenyl)magnesium bromide $(0.5 \mathrm{M}$ solution in THF, $13 \mathrm{~mL}, 6 \mathrm{mmol})$. Purification over silica gel $(20 \% \mathrm{v} / \mathrm{v}$ ethyl acetate-cyclohexane) gave the yellow oil product as an inseparable mixture of the corresponding di- and mono-N-Boc $\omega$-amino enones $(600 \mathrm{mg})$.

\section{TTOCT17-145}

tert-butyl (7-methyl-6-oxooct-7-en-1-yl)carbamate 3a<smiles>C=C(C)C(=O)CCCCCNC(=O)O</smiles>

$N$-Boc-caprolactam $1100 \mathrm{mg}, 5 \mathrm{mmol})$ was reacted with isopropenylmagnesium bromide $(1 \mathrm{M}$ solution in THF, $6 \mathrm{~mL}, 6$ $\mathrm{mmol})$. Purification over silica gel (20\% v/v ethyl acetate-cyclohexane) gave the product $(510 \mathrm{mg}, 40 \%$ yield) as a light yellow solid.

${ }^{1} \mathbf{H}$ NMR $\left(400 \mathrm{MHz}, \mathrm{CDCl}_{3}\right) \delta 5.94(\mathrm{~s}, 1 \mathrm{H}), 5.80-5.69(\mathrm{~m}, 1 \mathrm{H}), 4.52(\mathrm{~s}, 1 \mathrm{H}), 3.11(\mathrm{q}, \mathrm{J}=6.7 \mathrm{~Hz}, 2 \mathrm{H}), 2.68(\mathrm{t}, \mathrm{J}=7.3 \mathrm{~Hz}$, $2 \mathrm{H}), 1.86(\mathrm{t}, \mathrm{J}=1.2 \mathrm{~Hz}, 3 \mathrm{H}), 1.67-1.57(\mathrm{~m}, 3 \mathrm{H}), 1.54-1.45(\mathrm{~m}, 3 \mathrm{H}), 1.43(\mathrm{~s}, 9 \mathrm{H})$.

${ }^{13}$ C NMR $\left(100 \mathrm{MHz}, \mathrm{CDCl}_{3}\right) \delta 202.2,156.1,144.7,124.6,79.2,77.5,77.2,76.8,40.6,37.4,30.1,28.6,26.6,24.3,17.8$. MS (ESI) $\mathrm{m} / \mathrm{z}=278.2\left[\mathrm{M}+\mathrm{Na}^{+}\right]$ 
TTAUG18-109-3
tert-butyl (7-methylene-6-oxononyl)carbamate 3b

tert-butyl (7-methylene-6-oxononyl)carbamate 3b<smiles>C=C(CC)C(=O)CCCCCNC(=O)O</smiles>

$\mathrm{N}$-Boc-caprolactam $500 \mathrm{mg}, 2.3 \mathrm{mmol}$ ) was reacted with (1-methylenepropyl)magnesium bromide (1 M solution in THF, 4 $\mathrm{mL}, 4 \mathrm{mmol})$. Purification over silica gel (20\% v/v ethyl acetate-cyclohexane) gave the product (310 $\mathrm{mg}, 51 \%$ yield) as a light yellow solid.

${ }^{1} \mathbf{H}$ NMR $\left(400 \mathrm{MHz}, \mathrm{CDCl}_{3}\right) \delta 5.96(\mathrm{~s}, 1 \mathrm{H}), 5.70(\mathrm{t}, \mathrm{J}=1.6 \mathrm{~Hz}, 1 \mathrm{H}), 4.53(\mathrm{~s}, 1 \mathrm{H}), 3.11(\mathrm{q}, \mathrm{J}=6.8 \mathrm{~Hz}, 3 \mathrm{H}), 2.68(\mathrm{t}, \mathrm{J}=7.4$ $\mathrm{Hz}, 2 \mathrm{H}), 2.28$ (qt, J = 7.4, $1.2 \mathrm{~Hz}, 2 \mathrm{H}), 1.62(\mathrm{p}, \mathrm{J}=7.4 \mathrm{~Hz}, 2 \mathrm{H}), 1.53-1.45(\mathrm{~m}, 3 \mathrm{H}), 1.44(\mathrm{~s}, 9 \mathrm{H}), 1.36-1.28(\mathrm{~m}, 2 \mathrm{H}), 1.02$ $(\mathrm{t}, \mathrm{J}=7.4 \mathrm{~Hz}, 3 \mathrm{H})$.

\section{TTAUG18-112-3 \\ tert-butyl (7-methyl-6-oxonon-7-en-1-yl)carbamate 3c}<smiles>CC=C(C)C(=O)CCCCCNC(=O)O</smiles>

$N$-Boc-caprolactam $500 \mathrm{mg}, 2.3 \mathrm{mmol}$ ) was reacted with (but-2-en-2-yl)magnesium bromide (1 M solution in THF, $4 \mathrm{~mL}, 4$ $\mathrm{mmol})$. Purification over silica gel $(20 \% \mathrm{v} / \mathrm{v}$ ethyl acetate-cyclohexane) gave the product $(250 \mathrm{mg}, 41 \%$ yield $)$ as a light yellow solid.

${ }^{1} \mathbf{H}$ NMR $\left(400 \mathrm{MHz}, \mathrm{CDCl}_{3}\right) \delta 6.77-6.65\left(\mathrm{~m}, 1 \mathrm{H}, \mathrm{CCHCH}_{3}, E\right.$-isomer), 5.77 (qt, J = 7.3, $1.5 \mathrm{~Hz}, 1 \mathrm{H}, \mathrm{CCHCH}_{3} Z$-isomer), $4.53(\mathrm{~s}, 1 \mathrm{H}), 3.18-3.05(\mathrm{~m}, 2 \mathrm{H}), 2.63(\mathrm{t}, \mathrm{J}=7.4 \mathrm{~Hz}, 0 \mathrm{H}), 2.52(\mathrm{t}, 1 \mathrm{H}), 1.92-1.88(\mathrm{~m}, 2 \mathrm{H}), 1.86-1.80(\mathrm{~m}, 3 \mathrm{H}), 1.78-1.74$ $(\mathrm{m}, 1 \mathrm{H}), 1.68-1.57(\mathrm{~m}, 2 \mathrm{H}), 1.54-1.46(\mathrm{~m}, 2 \mathrm{H}), 1.43(\mathrm{~s}, 9 \mathrm{H}), 1.37-1.28(\mathrm{~m}, 2 \mathrm{H})$.

\section{Wz36b}

tert-butyl (8-methyl-6-oxonon-7-en-1-yl)carbamate 3d<smiles>CC(C)=CC(=O)CCCCCNC(=O)O</smiles>

$N$-Boc-caprolactam $1100 \mathrm{mg}, 5 \mathrm{mmol}$ ) was reacted with isopropenylmagnesium bromide (1 M solution in THF, $6 \mathrm{~mL}, 6$ $\mathrm{mmol})$. Purification over silica gel (20\% v/v ethyl acetate-cyclohexane) gave the yellow oil product as an inseparable mixture of the corresponding di- and mono-N-Boc $\omega$-amino enones $(550 \mathrm{mg})$

\subsubsection{Preparation of $N$-Boc $\omega$-amino ketones}

A Schlenk tube containing a solution of the corresponding $N$-Boc amino enone $(1 \mathrm{eq})$ and $5 \mathrm{~mol} \% \mathrm{Pd} / \mathrm{C}$ in $\mathrm{MeOH}(1$ $\mathrm{mL} \cdot \mathrm{mmol}^{-1}$ ) was bubbled with $\mathrm{N}_{2}$ for 30 mins to deoxygenate the reaction medium. The reaction vessel was then flushed with a balloon of $\mathrm{H}_{2}$ (g) before an additional balloon was added to maintain the $\mathrm{H}_{2}$ atmosphere. The reaction was stirred overnight subsequent to filtration through Celite which was then washed with a portion of EtOAc $(10 \mathrm{~mL})$. The filtered solution was then concentrated in vacuo to yield the corresponding $N$-Boc amino ketone product.

\section{TTOCT17-106 \\ tert-butyl (5-methyl-4-oxohexyl)carbamate 10a<smiles>CC(C)C(=O)CCCNC(=O)O</smiles>

$150 \mathrm{mg}$ of the corresponding mono and di $\mathrm{N}$-Boc amino enone was reduced with $\mathrm{H}_{2}$ over $\mathrm{Pd} / \mathrm{C}$ to yield the product as a clear oil $(65 \mathrm{mg})$.

${ }^{1} \mathbf{H}$ NMR $\left(400 \mathrm{MHz}, \mathrm{CDCl}_{3}\right) \delta 4.63(\mathrm{~s}, 1 \mathrm{H}), 3.09(\mathrm{q}, J=6.7 \mathrm{~Hz}, 2 \mathrm{H}), 2.57$ (hept, $\left.J=6.9 \mathrm{~Hz}, 1 \mathrm{H}\right), 2.47(\mathrm{t}, J=7.1 \mathrm{~Hz}, 2 \mathrm{H})$, $1.73(\mathrm{p}, J=7.0 \mathrm{~Hz}, 2 \mathrm{H}), 1.41(\mathrm{~s}, 9 \mathrm{H}), 1.06(\mathrm{~d}, J=6.9 \mathrm{~Hz}, 6 \mathrm{H})$.

${ }^{13} \mathrm{C}$ NMR $\left(100 \mathrm{MHz}, \mathrm{CDCl}_{3}\right) \delta 214.6,156.1,79.2,77.5,77.2,76.8,41.0,40.2,37.5,28.5,24.1,18.4$.

MS (ESI) $\mathrm{m} / \mathrm{z}=252.2\left[\mathrm{M}+\mathrm{Na}^{+}\right]$ 


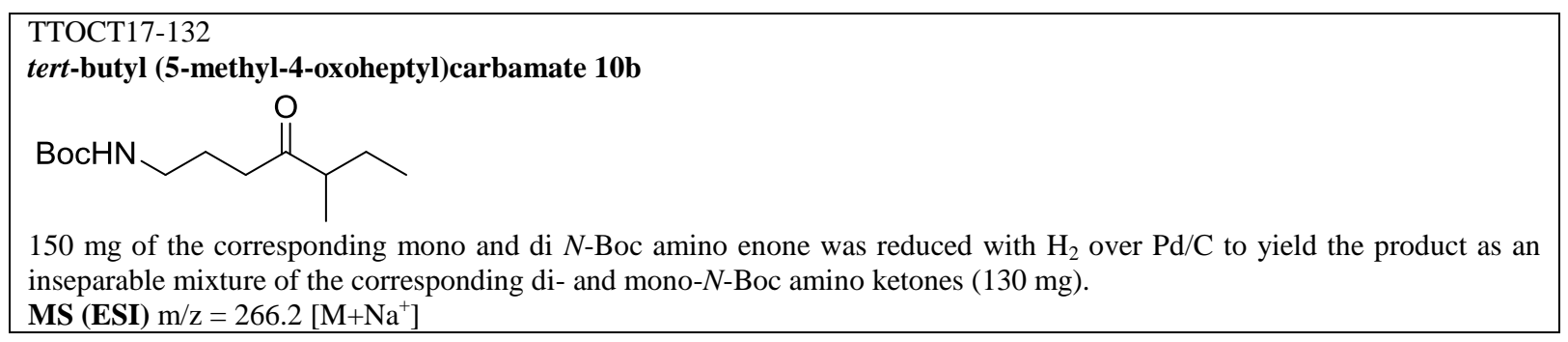

\section{TTAUG18-3}

tert-butyl (6-methyl-4-oxoheptyl)carbamate 10d

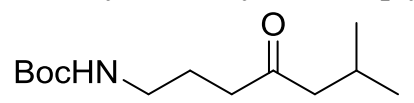

$30 \mathrm{mg}$ of the corresponding mono and di $\mathrm{N}$-Boc amino enone was reduced with $\mathrm{H}_{2}$ over $\mathrm{Pd} / \mathrm{C}$ to yield the product as an inseparable mixture of the corresponding di- and mono- $N$-Boc amino ketones $(25 \mathrm{mg})$.

MS (ESI) $\mathrm{m} / \mathrm{z}=266.2\left[\mathrm{M}+\mathrm{Na}^{+}\right]$

\section{TTOCT17-89}

tert-butyl (6-methyl-5-oxoheptyl)carbamate 11a<smiles>CC(C)C(=O)CCCCNC(=O)O</smiles>

$55 \mathrm{mg}$ of the corresponding $\mathrm{N}$-Boc amino enone was reduced with $\mathrm{H}_{2}$ over Pd/C to yield the product as a clear oil (49 $\mathrm{mg}$, $88 \%)$. Data is consistent with existing literature. ${ }^{\mathrm{S} 5}$

${ }^{1}$ H NMR $\left(400 \mathrm{MHz}, \mathrm{CDCl}_{3}\right) \delta 4.58(\mathrm{~s}, 1 \mathrm{H}), 3.10(\mathrm{q}, J=6.6 \mathrm{~Hz}, 2 \mathrm{H}), 2.57$ (hept, $\left.J=6.9 \mathrm{~Hz}, 1 \mathrm{H}\right), 2.46(\mathrm{t}, J=7.1 \mathrm{~Hz}, 2 \mathrm{H})$, $1.65-1.51(\mathrm{~m}, 2 \mathrm{H}), 1.50-1.44(\mathrm{~m}, 2 \mathrm{H}), 1.42(\mathrm{~s}, 9 \mathrm{H}), 1.07(\mathrm{~d}, J=6.9 \mathrm{~Hz}, 6 \mathrm{H})$.

${ }^{13}$ C NMR (100 MHz, $\left.\mathrm{CDCl}_{3}\right) \delta 214.7,156.1,79.2,77.5,77.2,76.8,41.0,40.3,39.9,29.7,28.5,20.8,18.4$.

MS (ESI) $\mathrm{m} / \mathrm{z}=266.2\left[\mathrm{M}+\mathrm{Na}^{+}\right]$

\section{TTOCT17-132 \\ tert-butyl (6-methyl-5-oxooctyl)carbamate 11b}<smiles>CCC(C)C(=O)CCCCNC(=O)O</smiles>

$150 \mathrm{mg}$ of the corresponding $\mathrm{N}$-Boc amino enone was reduced with $\mathrm{H}_{2}$ over $\mathrm{Pd} / \mathrm{C}$ to the product as a clear oil (130 mg, $87 \%)$.

${ }^{1} \mathbf{H}$ NMR $\left(400 \mathrm{MHz}, \mathrm{CDCl}_{3}\right) \delta 4.59(\mathrm{~s}, 1 \mathrm{H}), 3.10(\mathrm{q}, J=6.6 \mathrm{~Hz}, 2 \mathrm{H}), 2.73-2.21(\mathrm{~m}, 3 \mathrm{H}), 1.80-1.52(\mathrm{~m}, 3 \mathrm{H}), 1.49-1.30$ $(\mathrm{m}, 12 \mathrm{H}), 1.04(\mathrm{~d}, J=6.9 \mathrm{~Hz}, 3 \mathrm{H}), 0.85(\mathrm{t}, J=7.4 \mathrm{~Hz}, 3 \mathrm{H})$.

${ }^{13}$ C NMR $\left(100 \mathrm{MHz}, \mathrm{CDCl}_{3}\right) \delta 214.8,156.1,79.2,48.0,40.6,40.3,29.7,28.5,26.1,20.7,16.1,11.8$.

MS (ESI) $\mathrm{m} / \mathrm{z}=280.2\left[\mathrm{M}+\mathrm{Na}^{+}\right]$

\section{TTOCT17-157}

tert-butyl-(6-methyl-5-oxooctyl)carbamate 11b<smiles>CCC(C)C(=O)CCCCCNC(=O)O</smiles>

$50 \mathrm{mg}$ of the corresponding $N$-Boc amino ketone was reacted with TFA to yield the product as a light yellow oil (47 mg, $93 \%)$.

${ }^{1} \mathbf{H}$ NMR $\left(400 \mathrm{MHz}, \mathrm{CDCl}_{3}\right) \delta 4.52(\mathrm{~s}, 1 \mathrm{H}), 3.10(\mathrm{q}, \mathrm{J}=6.9 \mathrm{~Hz}, 2 \mathrm{H}), 2.51-2.37(\mathrm{~m}, 3 \mathrm{H}), 1.72-1.53(\mathrm{~m}, 4 \mathrm{H}), 1.52-1.45$ $(\mathrm{m}, 2 \mathrm{H}), 1.44(\mathrm{~s}, 9 \mathrm{H}), 1.41-1.24(\mathrm{~m}, 3 \mathrm{H}), 1.05(\mathrm{~d}, \mathrm{~J}=6.9 \mathrm{~Hz}, 3 \mathrm{H}), 0.86(\mathrm{t}, \mathrm{J}=7.4 \mathrm{~Hz}, 2 \mathrm{H})$.

${ }^{13}$ C NMR $\left(100 \mathrm{MHz}, \mathrm{CDCl}_{3}\right) \delta 214.9,156.1,79.3,48.0,41.1,30.1,28.6,28.2,26.6,26.1,23.4,16.1,11.9$. 


\subsubsection{Preparation of cyclic enimines and imines}

TFA (5 eq) was added neat to the corresponding $N$-Boc amino enones or $N$-Boc amino ketones ( 1 eq) at $0{ }^{\circ} \mathrm{C}$ and was allowed to warm to room temperature and stirred for a further $2 \mathrm{~h}$. Excess TFA was removed at reduced pressure without quenching to reveal the TFA salt which was characterised with no further purification.* Alternatively the reaction was quenched at room temperature with $\mathrm{NaCHO}_{3}$ (aq, sat., $\left.10 \mathrm{~mL} \cdot \mathrm{mmol}^{-1}\right)$, before being extracted into DCM $(3 \times 10 \mathrm{~mL})$. The combined fractions were dried with magnesium sulphate, filtered and then acidified with $\mathrm{HCl}\left(2 \mathrm{M}\right.$ solution in $\left.\mathrm{Et}_{2} \mathrm{O}, 2 \mathrm{eq}\right)$ subsequent to concentration under reduced pressure to reveal the corresponding hydrochloride salt.

*NMR spectra of TFA salts $1 \mathrm{~h}$ after sample preparation contained duplicate peaks for all predicted protons. Presented spectra are taken after $12 \mathrm{~h}$ and contain a single peak for each predicted proton.

\subsection{Enimines}

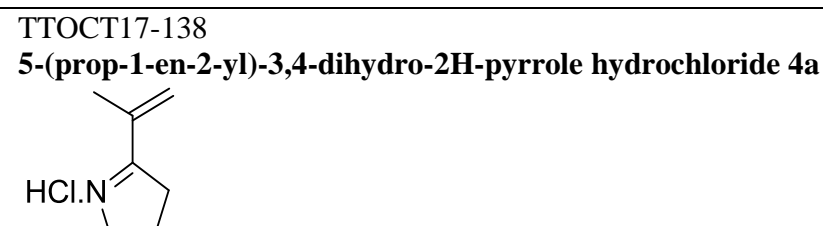

$100 \mathrm{mg}$ of the corresponding $\mathrm{N}$-Boc amino enone was reacted with TFA to yield the product as a light yellow solid (65 mg, quantitative).

${ }^{1}$ H NMR $\left(400 \mathrm{MHz}, \mathrm{CD}_{3} \mathrm{OD}\right) \delta 6.37(\mathrm{~s}, 1 \mathrm{H}), 6.29(\mathrm{~s}, 1 \mathrm{H}), 4.18(\mathrm{t}, J=7.4 \mathrm{~Hz}, 2 \mathrm{H}), 3.41(\mathrm{t}, J=7.8 \mathrm{~Hz}, 2 \mathrm{H}), 2.35(\mathrm{~h}, J=5.6$, $4.3 \mathrm{~Hz}, 2 \mathrm{H}), 2.15$ (s, 3H).

${ }^{13}$ C NMR (100 MHz, CD $\left.\mathrm{CD}_{3} \mathrm{OD}\right) \delta 187.8,138.1,135.3,55.1,35.3,21.1,18.3$.

GC-MS (EI) $\mathrm{m} / \mathrm{z}=109$
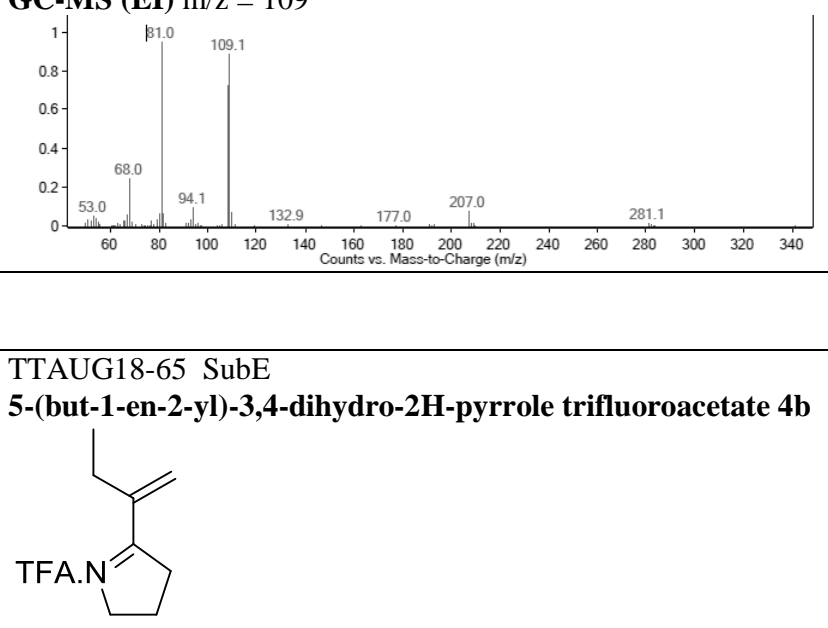

$90 \mathrm{mg}$ of the corresponding $N$-Boc amino enone was reacted with TFA to yield the product as a dark brown oil $(82 \mathrm{mg}$, quantitative).

${ }^{1}$ H NMR $\left(400 \mathrm{MHz}, \mathrm{CDCl}_{3}\right) \delta 6.29(\mathrm{~s}, 1 \mathrm{H}), 6.21(\mathrm{~s}, 1 \mathrm{H}), 4.30-4.13(\mathrm{~m}, 2 \mathrm{H}), 3.32(\mathrm{tt}, \mathrm{J}=8.3,2.0 \mathrm{~Hz}, 2 \mathrm{H}), 2.57-2.26(\mathrm{~m}$, $4 \mathrm{H}), 1.17(\mathrm{t}, \mathrm{J}=7.3 \mathrm{~Hz}, 3 \mathrm{H})$.

${ }^{13}$ C NMR $\left(100 \mathrm{MHz}, \mathrm{CDCl}_{3}\right) \delta 185.2,139.8,135.2,54.1,34.6,23.7,19.8,11.4$.

GC-MS (EI) $\mathrm{m} / \mathrm{z}=123$

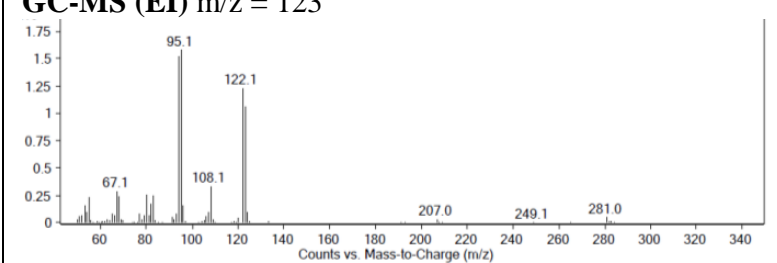




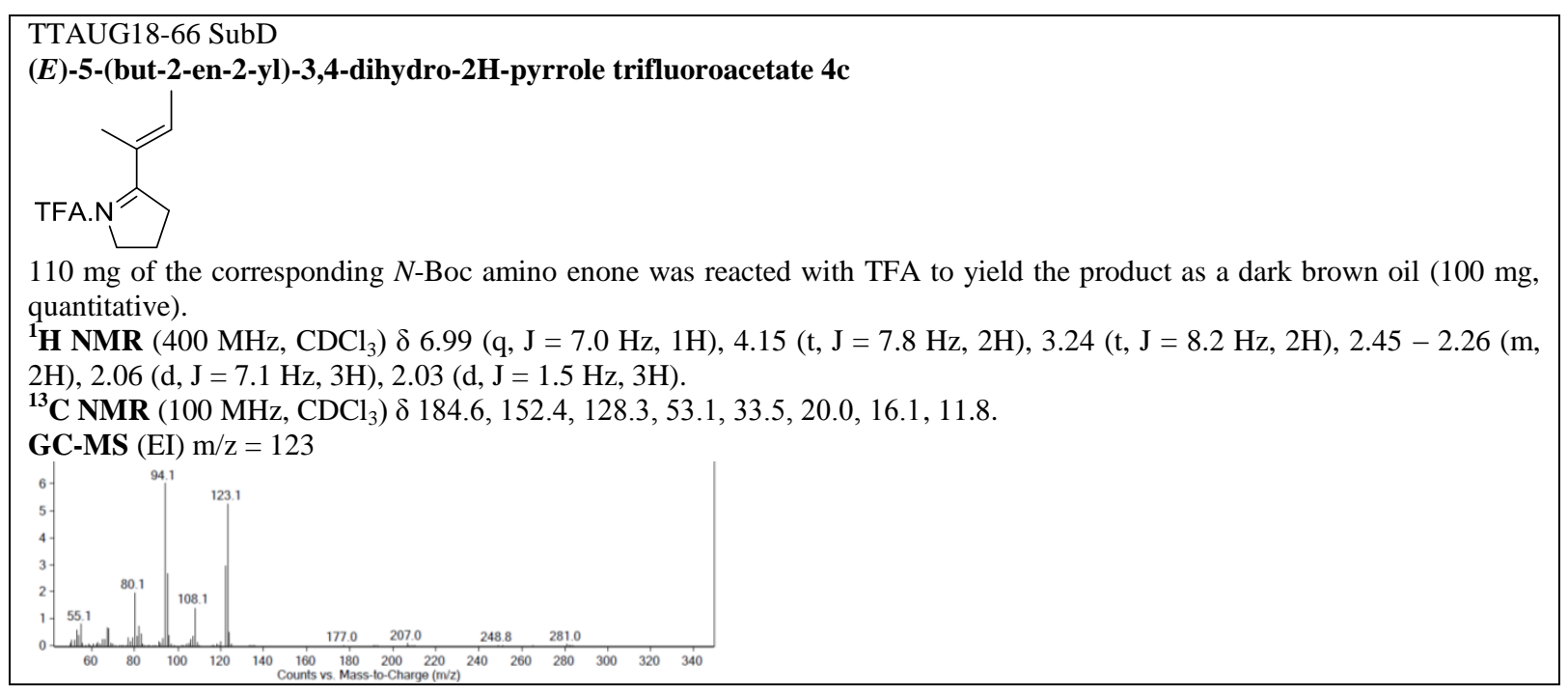

\section{TTOCT17-111}

5-(2-methylprop-1-en-1-yl)-3,4-dihydro-2H-pyrrole hydrochloride 4d<smiles>CC(C)=CC1=NCCC1</smiles>

$100 \mathrm{mg}$ of the corresponding $\mathrm{N}$-Boc amino enone was reacted with TFA to yield the product as a light yellow solid (50 mg, $77 \%)$.

${ }^{1} \mathbf{H}$ NMR $\left(400 \mathrm{MHz}, \mathrm{CD}_{3} \mathrm{OD}\right) \delta 6.47(\mathrm{~s}, 1 \mathrm{H}), 3.99(\mathrm{t}, J=7.3 \mathrm{~Hz}, 2 \mathrm{H}), 3.41(\mathrm{t}, J=7.5 \mathrm{~Hz}, 2 \mathrm{H}), 2.36(\mathrm{p}, J=8.4,8.0 \mathrm{~Hz}, 2 \mathrm{H})$, $2.25(\mathrm{~s}, 3 \mathrm{H}), 2.18(\mathrm{~s}, 3 \mathrm{H})$.

${ }^{13}$ C NMR (100 MHz, $\left.\mathrm{CD}_{3} \mathrm{OD}\right) \delta 184.9,169.6,116.5,52.1,37.4,29.8,22.3,21.9$.

GC-MS (EI) $\mathrm{m} / \mathrm{z}=123$

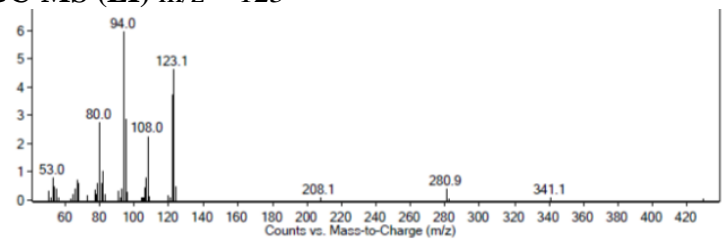

\section{TTAUG18-38}

6-(prop-1-en-2-yl)-2,3,4,5-tetrahydropyridine hydrochloride 5a<smiles>C=C(C)C1=NCCCC1</smiles>

$280 \mathrm{mg}$ of the corresponding $N$-Boc amino enone was reacted with TFA to yield the product as a dark orange oil (325 mg, quant).

${ }^{1} \mathbf{H}$ NMR $\left(400 \mathrm{MHz}, \mathrm{CDCl}_{3}\right) \delta 6.16(\mathrm{~s}, 1 \mathrm{H}), 5.99(\mathrm{~d}, \mathrm{~J}=1.6 \mathrm{~Hz}, 1 \mathrm{H}), 3.83(\mathrm{dt}, \mathrm{J}=6.6,3.5 \mathrm{~Hz}, 2 \mathrm{H}), 3.01-2.87(\mathrm{~m}, 2 \mathrm{H}), 2.13$ $(\mathrm{d}, \mathrm{J}=1.4 \mathrm{~Hz}, 3 \mathrm{H}), 1.95(\mathrm{p}, \mathrm{J}=3.0 \mathrm{~Hz}, 4 \mathrm{H})$.

${ }^{13}$ C NMR $\left(100 \mathrm{MHz}, \mathrm{CDCl}_{3}\right) \delta 180.4,137.9,131.4,45.5,26.6,19.4,18.0,17.3$.

GC-MS (EI) $\mathrm{m} / \mathrm{z}=123$

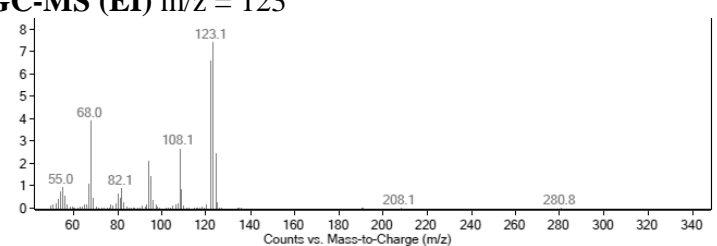

HRMS $\mathrm{C}_{8} \mathrm{H}_{15} \mathrm{~N}^{+}$calcd. $=124.1121$, found $=124.1117$ 

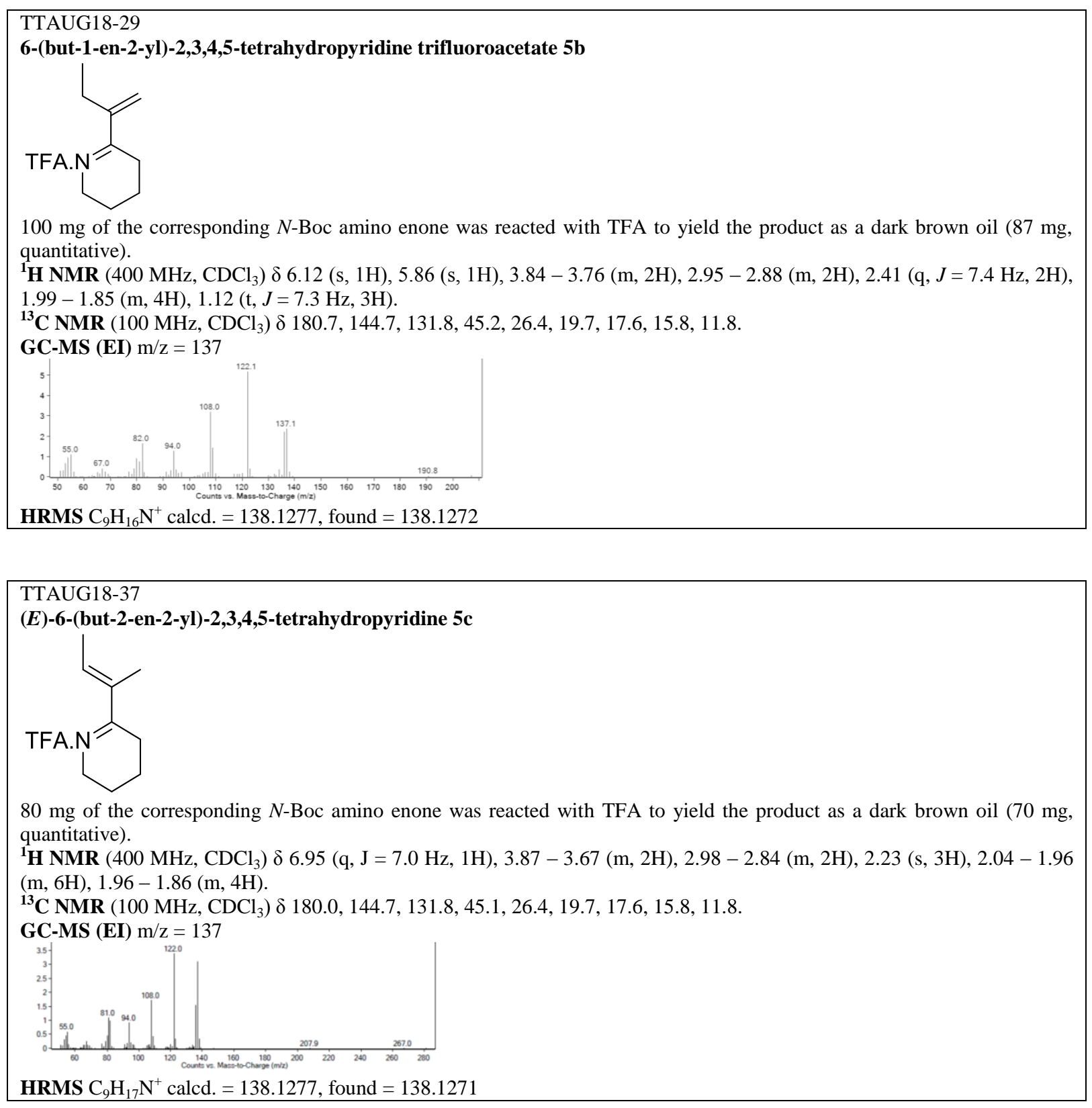

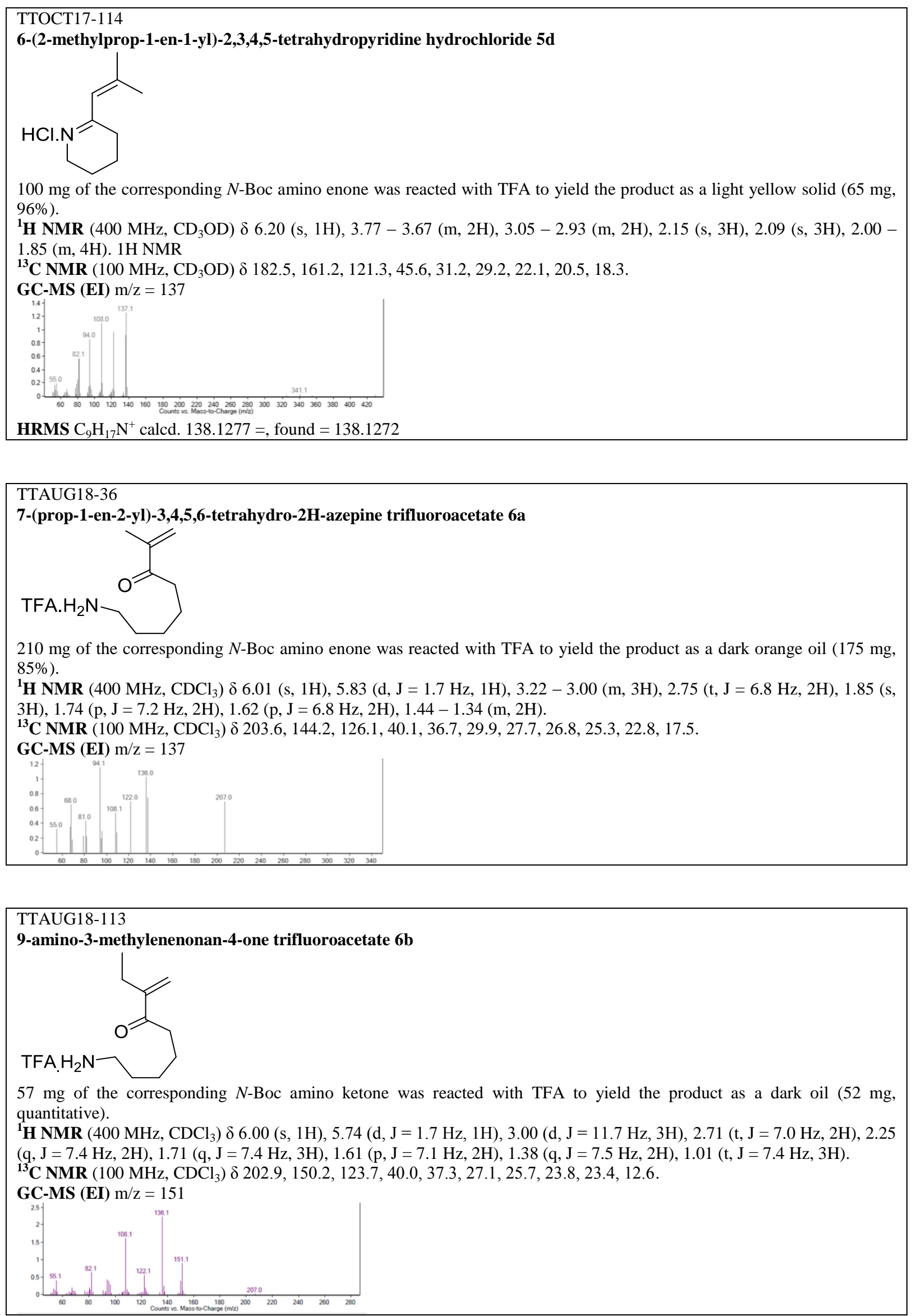


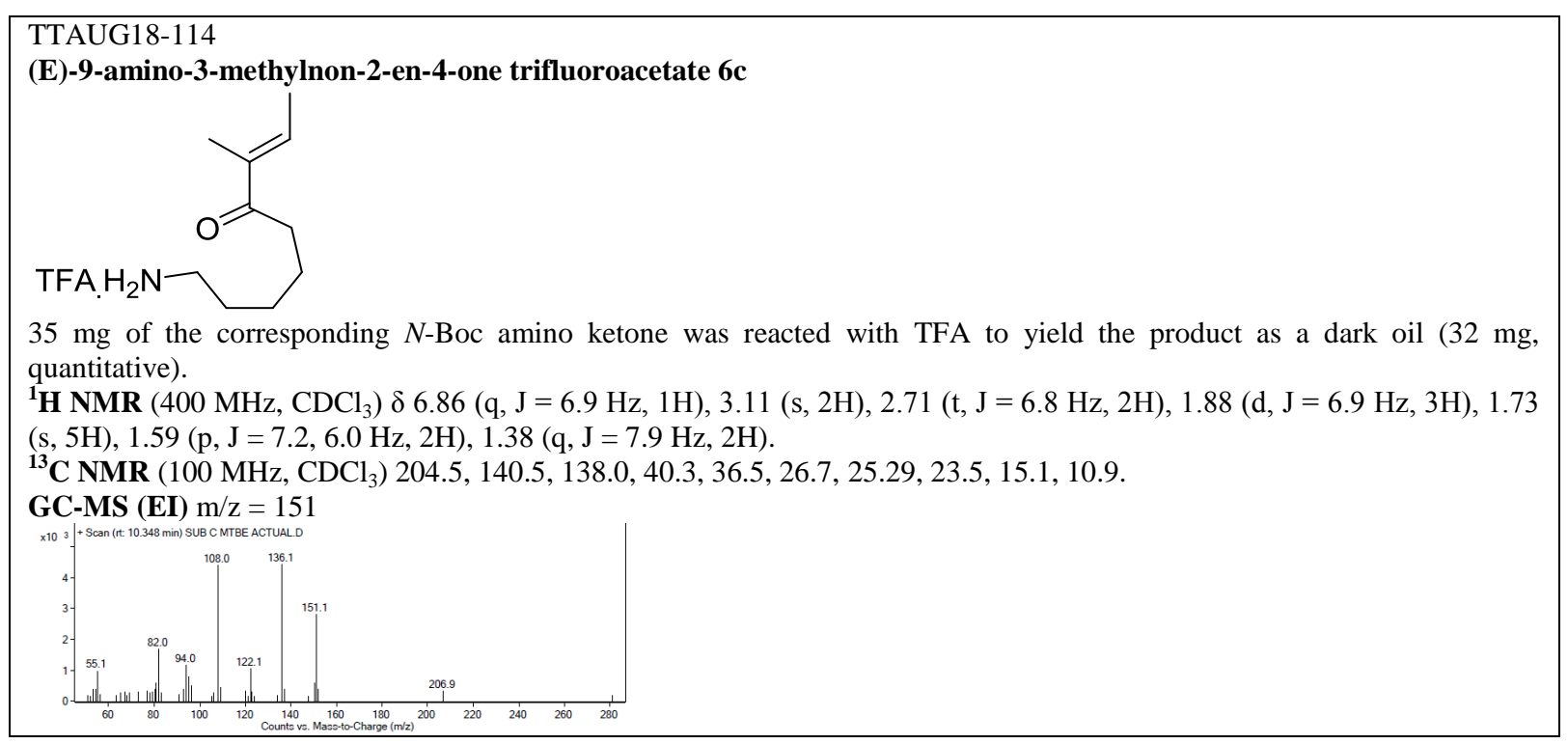

\section{TTAUG18-96 \\ 9-amino-2-methylnon-2-en-4-one trifluoroacetate 6d}<smiles>CC(C)=CC(=O)CCCCCN</smiles>

$120 \mathrm{mg}$ of the corresponding $N$-Boc amino ketone was reacted with TFA to yield the product as a a dark oil (110 mg, quantitative).

${ }^{1}$ H NMR $\left(400 \mathrm{MHz}, \mathrm{CD}_{3} \mathrm{OD}\right) \delta 6.11(\mathrm{~s}, 1 \mathrm{H}), 3.07(\mathrm{~d}, \mathrm{~J}=39.2 \mathrm{~Hz}, 2 \mathrm{H}), 2.47(\mathrm{~d}, \mathrm{~J}=6.2 \mathrm{~Hz}, 2 \mathrm{H}), 2.12(\mathrm{~s}, 3 \mathrm{H}), 1.92(\mathrm{~s}, 3 \mathrm{H})$, $1.72(\mathrm{~s}, 2 \mathrm{H}), 1.60(\mathrm{~d}, \mathrm{~J}=15.8 \mathrm{~Hz}, 2 \mathrm{H}), 1.38(\mathrm{~s}, 2 \mathrm{H})$

${ }^{13}$ C NMR (100 MHz, CD 3 OD) $\delta 203.5,159.1,123.4,43.4,40.2,28.0,26.7,25.2,22.8,21.1$.

GC-MS (EI) $\mathrm{m} / \mathrm{z}=151$

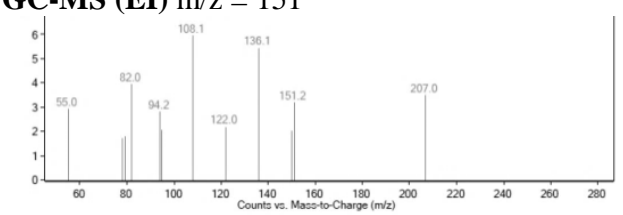

\subsection{Imines}

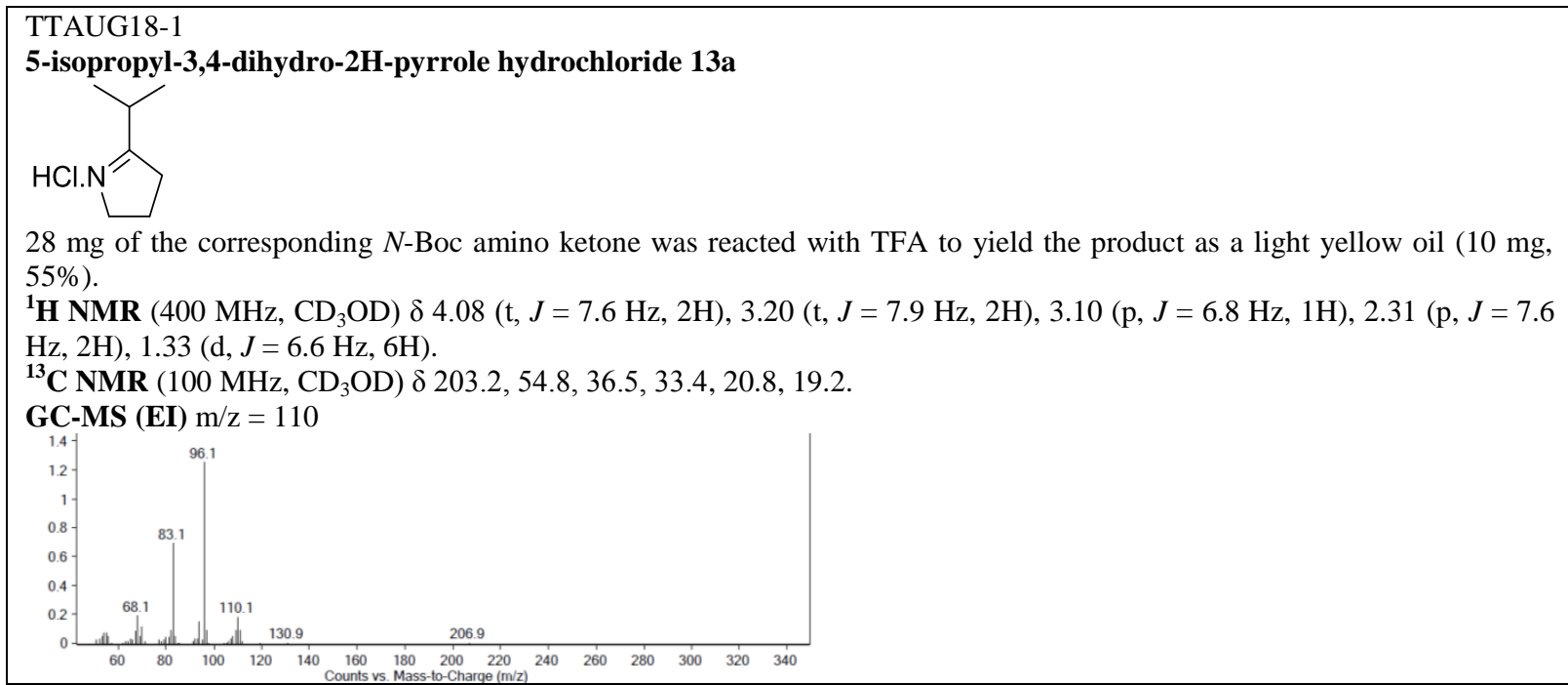




\section{TTAUG18-68 \\ 5-(sec-butyl)-3,4-dihydro-2H-pyrrole hydrochloride 13b}<smiles>CCC(C)C1=NCCC1</smiles>

$54 \mathrm{mg}$ of the corresponding $\mathrm{N}$-Boc amino ketone was reacted with TFA to yield the product as a light yellow oil (34 mg, $90 \%)$.

${ }^{1} \mathbf{H}$ NMR $\left(400 \mathrm{MHz}, \mathrm{CDCl}_{3}\right) \delta 4.16(\mathrm{~s}, 2 \mathrm{H}), 3.41(\mathrm{~s}, 1 \mathrm{H}), 3.03(\mathrm{~s}, 2 \mathrm{H}), 2.32(\mathrm{~s}, 2 \mathrm{H}), 1.79-1.62(\mathrm{~m}, 2 \mathrm{H}), 1.32(\mathrm{~d}, \mathrm{~J}=5.1 \mathrm{~Hz}$, $3 \mathrm{H}), 0.99(\mathrm{t}, \mathrm{J}=6.7 \mathrm{~Hz}, 3 \mathrm{H})$.

${ }^{13}$ C NMR $\left(100 \mathrm{MHz}, \mathrm{CDCl}_{3}\right) \delta 198.66,53.81,38.15,34.37,27.75,20.02,17.64,11.93$.

GC-MS (EI) $\mathrm{m} / \mathrm{z}=124$

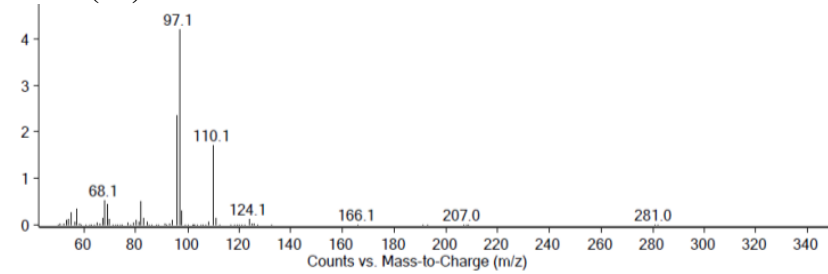

\section{TTAUG2018-7}

5-isobutyl-3,4-dihydro-2H-pyrrole hydrochloride 13d<smiles>CC(C)CC1=NCCC1</smiles>

$44 \mathrm{mg}$ of the corresponding $\mathrm{N}$-Boc amino ketone was reacted with TFA to yield the product as a light yellow oil (27 mg, 93\%).

${ }^{1} \mathbf{H}$ NMR $\left(400 \mathrm{MHz}, \mathrm{CD}_{3} \mathrm{OD}\right) \delta 4.10(\mathrm{t}, J=7.7 \mathrm{~Hz}, 2 \mathrm{H}), 3.18(\mathrm{t}, J=8.1 \mathrm{~Hz}, 2 \mathrm{H}), 2.71(\mathrm{~d}, J=7.1 \mathrm{~Hz}, 2 \mathrm{H}), 2.32(\mathrm{p}, J=7.6$ $\mathrm{Hz}, 2 \mathrm{H}), 2.17(\mathrm{dt}, J=13.4,6.7 \mathrm{~Hz}, 1 \mathrm{H}), 1.03(\mathrm{~d}, J=6.6 \mathrm{~Hz}, 6 \mathrm{H})$.

${ }^{13}$ C NMR $\left(100 \mathrm{MHz}, \mathrm{CD}_{3} \mathrm{OD}\right) \delta 198.8,54.6,41.7,38.3,27.7,22.7,21.0$.

GC-MS (EI) $\mathrm{m} / \mathrm{z}=124$

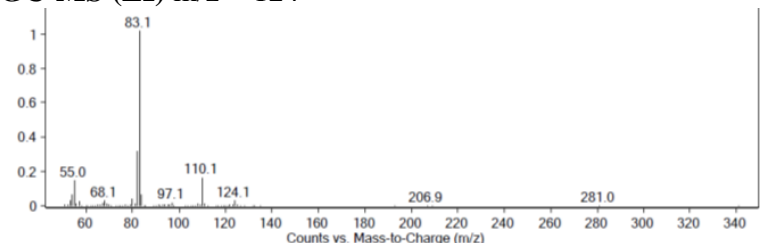

\section{6-isopropyl-2,3,4,5-tetrahydropyridine hydrochloride 14a}

TTOCT17-96<smiles>CC(C)C1=NCCCC1</smiles>

$49 \mathrm{mg}$ of the corresponding $\mathrm{N}$-Boc amino ketone was reacted with TFA to yield the product as a light yellow oil (16 mg, 49\%). Data is consistent with existing literature. ${ }^{\mathrm{S} .}$

${ }^{1} \mathbf{H}$ NMR $\left(400 \mathrm{MHz}, \mathrm{CD}_{3} \mathrm{OD}\right) \delta 3.49(\mathrm{t}, \mathrm{J}=5.9 \mathrm{~Hz}, 2 \mathrm{H}), 2.83-2.71(\mathrm{~m}, 1 \mathrm{H}), 2.71-2.64(\mathrm{~m}, 2 \mathrm{H}), 1.79-1.61(\mathrm{~m}, 4 \mathrm{H}), 1.10$ $(\mathrm{d}, \mathrm{J}=6.9 \mathrm{~Hz}, 6 \mathrm{H})$.

${ }^{13}$ C NMR (100 MHz, CD $\left.{ }_{3} \mathrm{OD}\right) \delta 198.4,46.0,38.6,28.2,20.4,19.3,17.8$.

GC-MS (EI) $\mathrm{m} / \mathrm{z}=125$

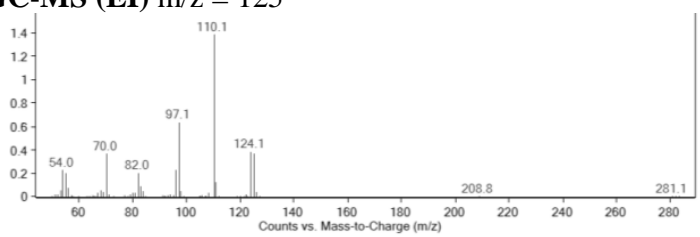



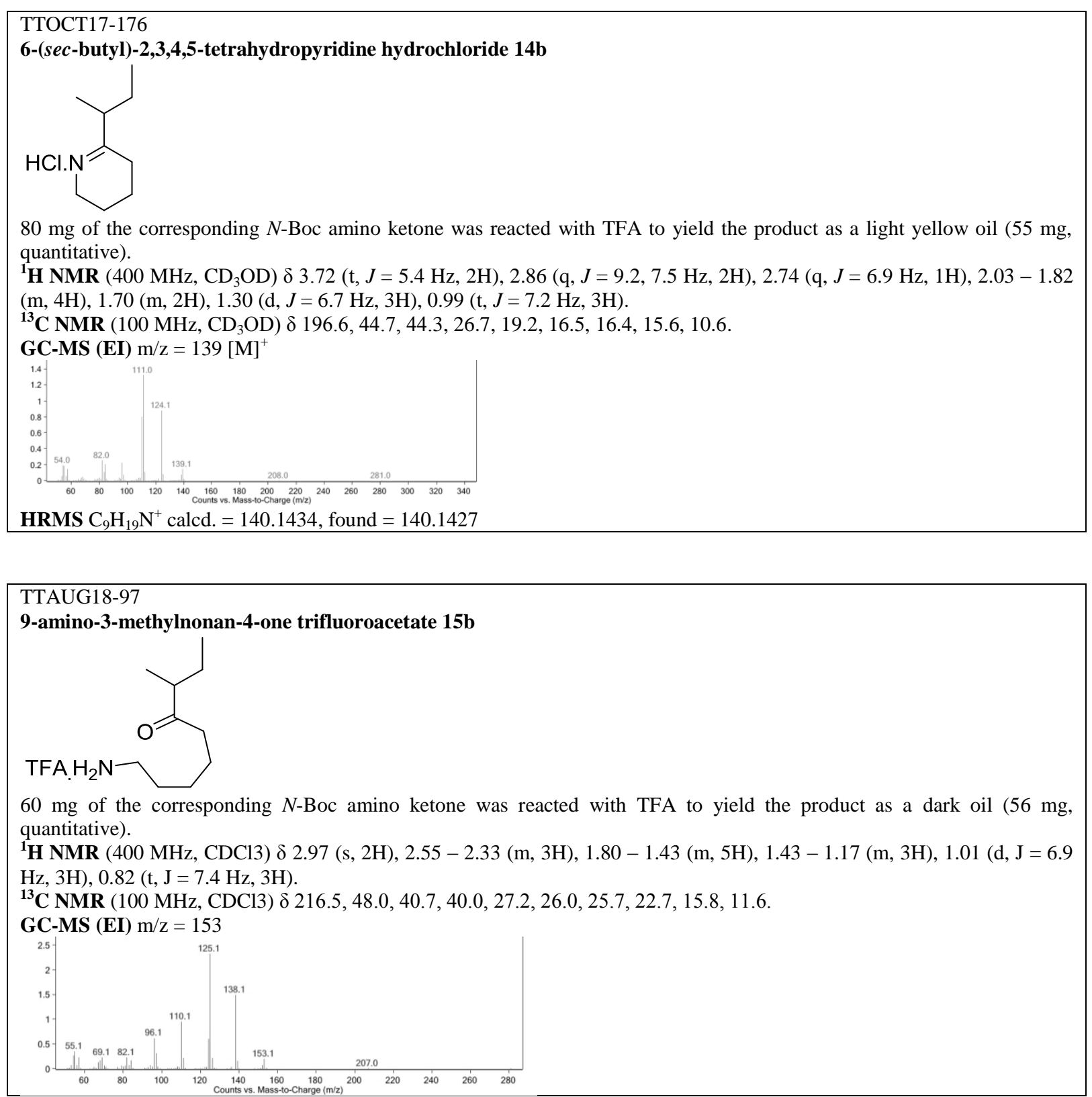

\subsubsection{Preparation of cyclic amines}

Procedure A. Sodium borohydride (2 eq) was added to the corresponding cyclic imine or enimine (1 eq) solution in $20 \% \mathrm{v} / \mathrm{v}$ $\mathrm{dH}_{2} \mathrm{O}$ in $\mathrm{MeOH}\left(30 \mathrm{~mL} \cdot \mathrm{mol}^{-1}\right)$ and left overnight at r.t. before being quenched with aqueous $\mathrm{HCl}(1 \mathrm{M})$ until pH 1 and left to stir for 15 mins. The mixture was then adjusted to $\mathrm{pH}>12$ using aqueous $\mathrm{NaOH}(5 \mathrm{M})$ before being extracted into DCM (3 $\mathrm{x} 10 \mathrm{~mL}$ ). The combined fractions were then dried with magnesium sulphate, filtered and resulting solution was acidified with $\mathrm{HCl}\left(2 \mathrm{M}\right.$ solution in $\left.\mathrm{Et}_{2} \mathrm{O}, 2 \mathrm{eq}\right)$ followed by concentration under reduced pressure to reveal the racemic amine hydrochloride salt.

Procedure B. A Schlenk tube containing a solution of the corresponding cyclic enimine ( $1 \mathrm{eq}$ ) and $5 \mathrm{~mol} \% \mathrm{Pd} / \mathrm{C}$ in $\mathrm{MeOH}$ $\left(1 \mathrm{~mL} \cdot \mathrm{mmol}^{-1}\right)$ was bubbled with $\mathrm{N}_{2}$ for 30 mins to deoxygenate the reaction medium. The reaction vessel was then flushed with a balloon of $\mathrm{H}_{2}(\mathrm{~g})$ before another was attached to maintain the $\mathrm{H}_{2}$ atmosphere. The reaction was stirred overnight subsequent to filtration through a Celite pad which was then washed with a portion of EtOAc $(10 \mathrm{~mL})$. The filtered solution was then concentrated under vacuo to yield the corresponding cyclic amine. 

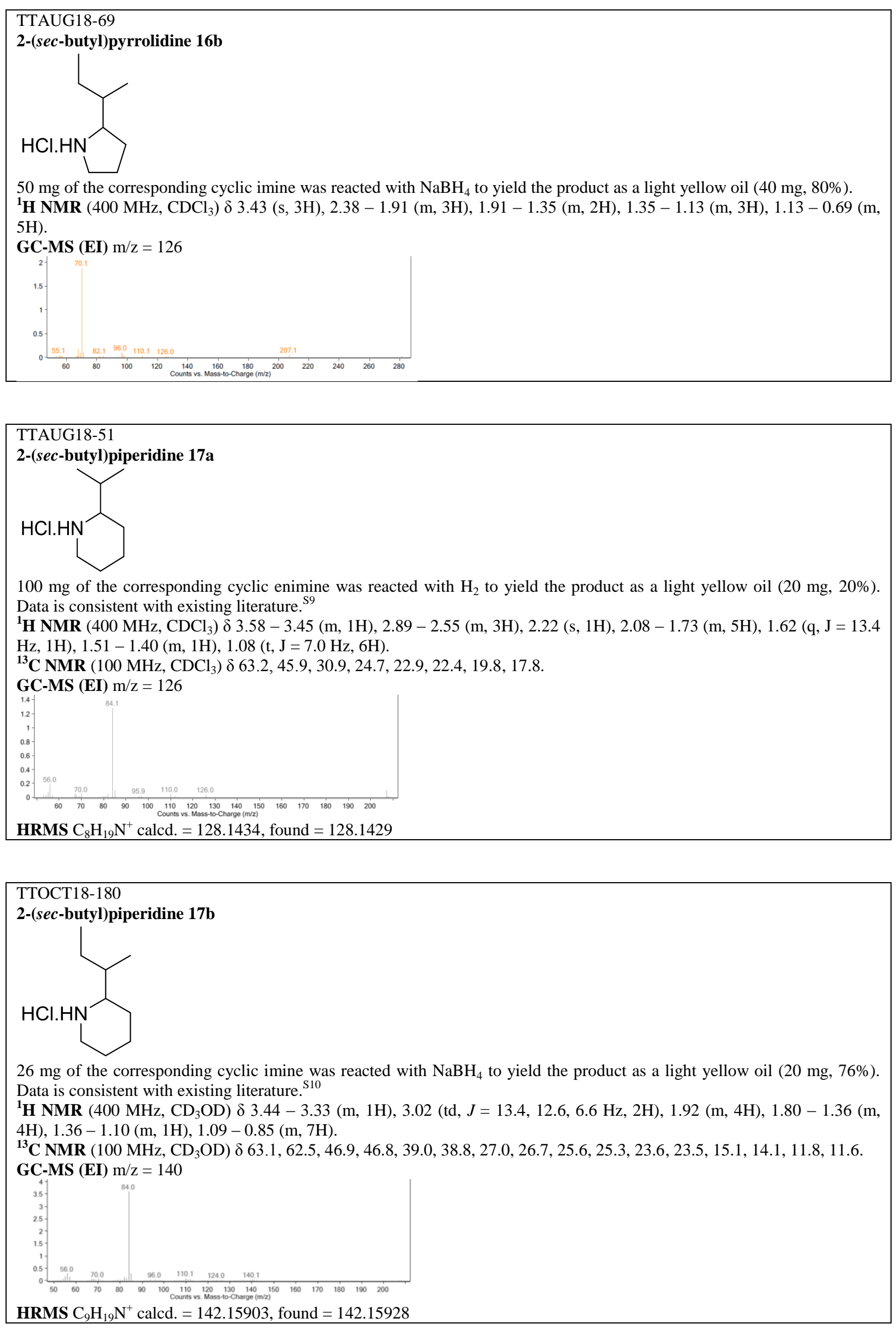


\section{6-isobutyl-2,3,4,5-tetrahydropyridine 14d}<smiles>CC(C)CC1=NCCCC1</smiles>

GC-MS (EI) $\mathrm{m} / \mathrm{z}=139$

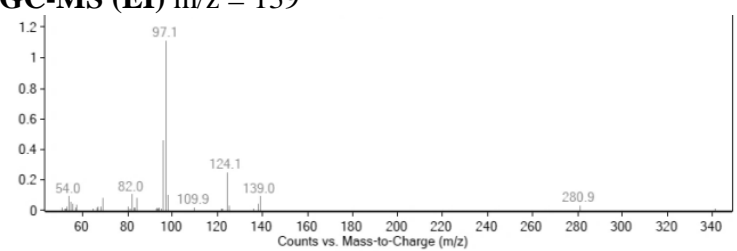

\section{7-isopropyl-3,4,5,6-tetrahydro-2H-azepine 15a}<smiles>CC(C)C1=NCCCCC1</smiles>

GC-MS (EI) $\mathrm{m} / \mathrm{z}=139$

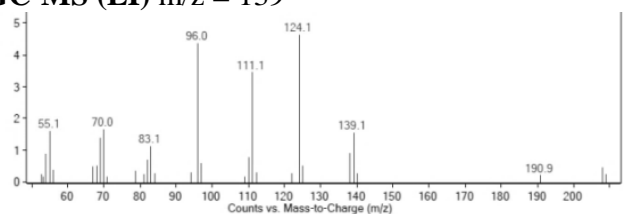

\section{7-isobutyl-3,4,5,6-tetrahydro-2H-azepine 15d}<smiles>CC(C)CC1=NCCCCC1</smiles>

GC-MS (EI) $\mathrm{m} / \mathrm{z}=153$

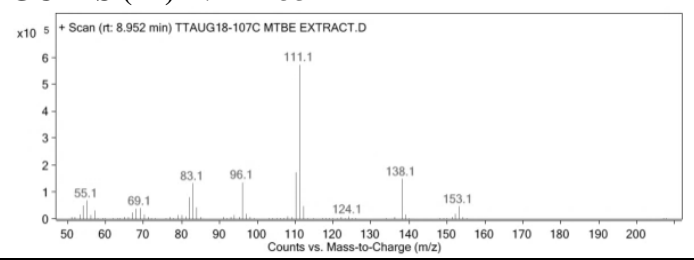



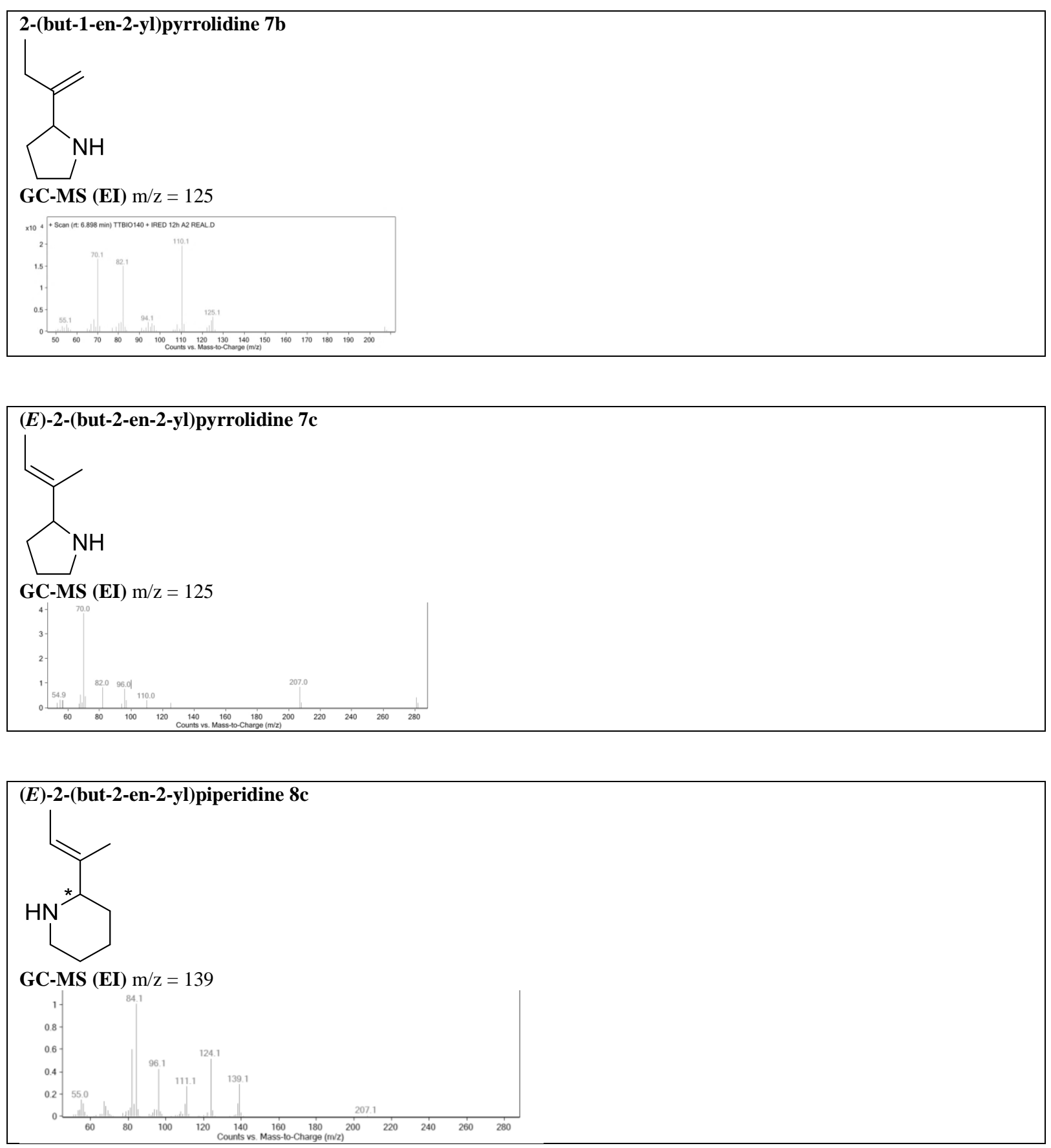

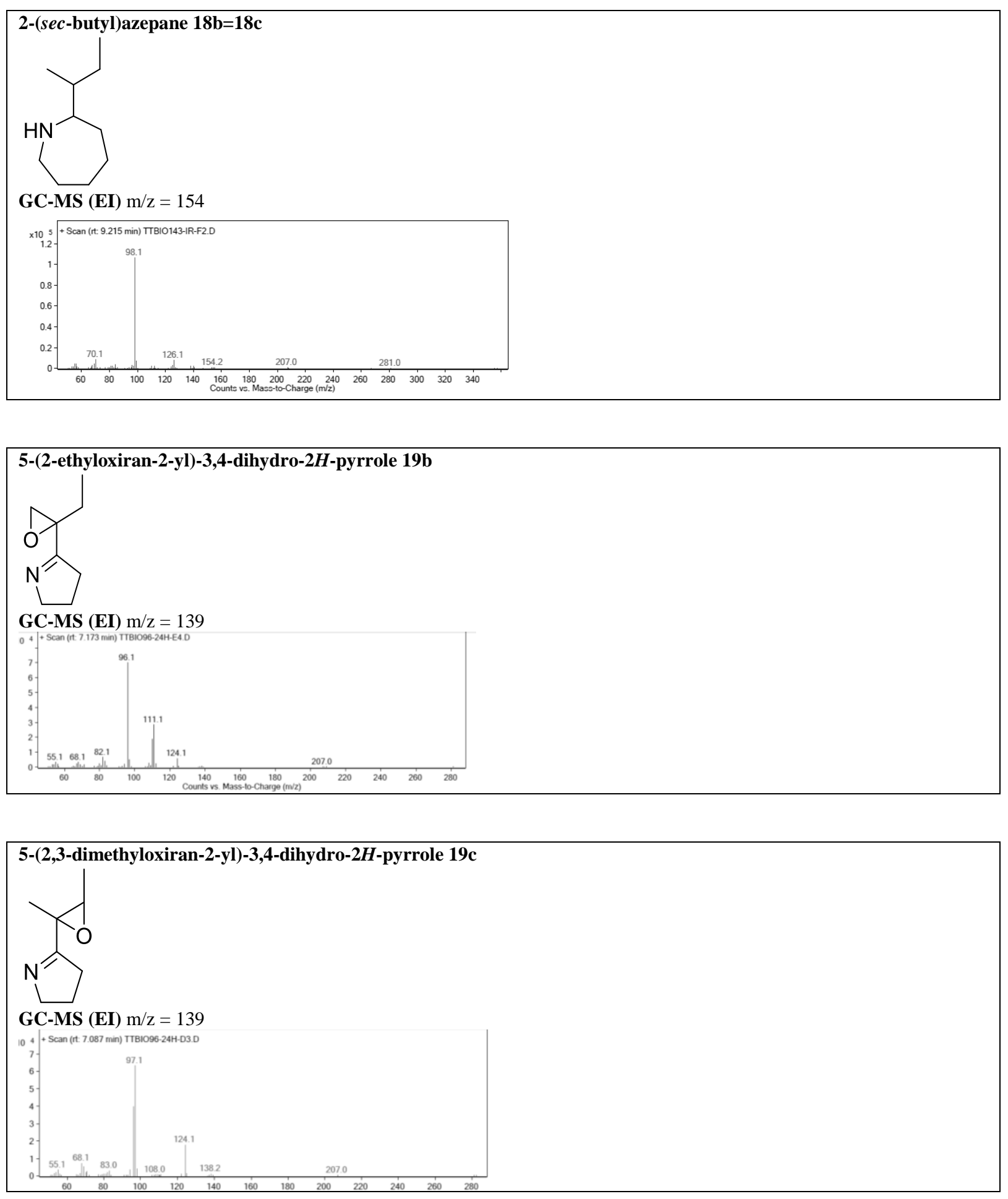


\section{6-(2,3-dimethyloxiran-2-yl)-2,3,4,5-tetrahydropyridine 20c}<smiles>CC1CC1(C)C1=NCCCC1</smiles>

GC-MS (EI) $\mathrm{m} / \mathrm{z}=153$

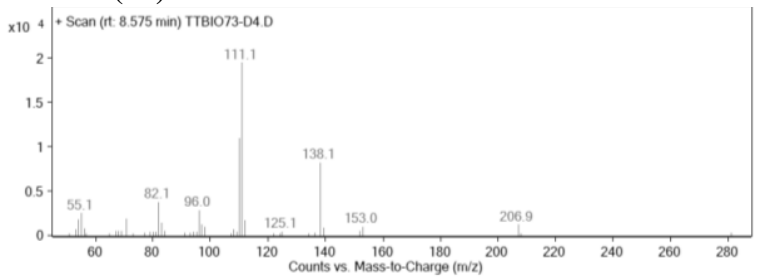

7-(2-ethyloxiran-2-yl)-3,4,5,6-tetrahydro-2H-azepine 21b<smiles>CCC1(C2=NCCCCC2)CO1</smiles>

GC-MS (EI) $\mathrm{m} / \mathrm{z}=167$

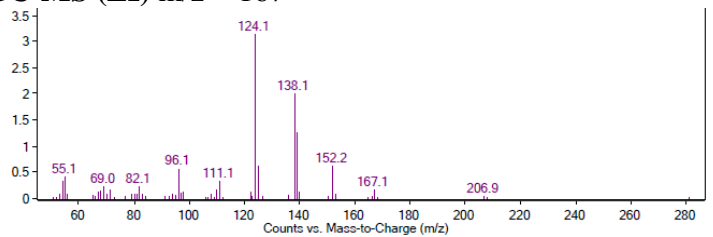

7-(2,3-dimethyloxiran-2-yl)-3,4,5,6-tetrahydro- $2 \mathrm{H}$-azepine 21c

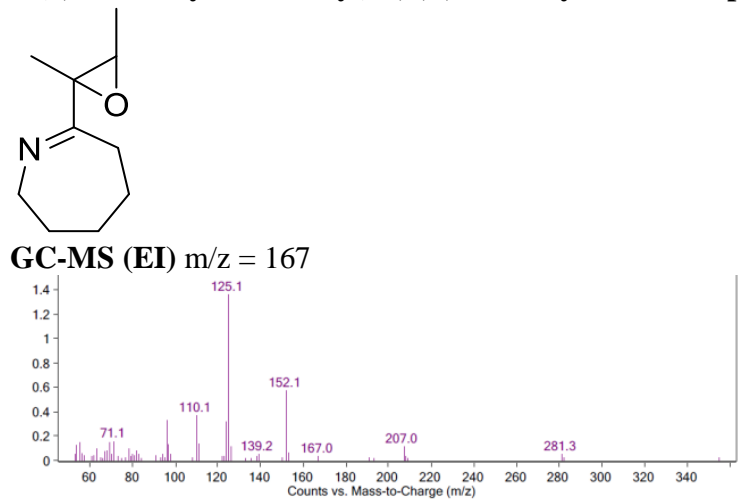




\subsection{GC-FID}

Method A: Agilent CP-ChiraSil-DEX CB column $\left(25 \mathrm{~m} \times 0.25 \mathrm{~mm} \times 0.25 \mu \mathrm{m}\right.$, Method: $1.2 \mathrm{~mL} \cdot \mathrm{min}^{-1}, 50{ }^{\circ} \mathrm{C}$ to $250{ }^{\circ} \mathrm{C}$ at 5 ${ }^{\circ} \mathrm{C} \cdot \mathrm{min}^{-1}$, injector $200{ }^{\circ} \mathrm{C}$, detector $250{ }^{\circ} \mathrm{C}$ )

Method B: HP-1 column $\left(30 \mathrm{~m} \times 0.32 \mathrm{~mm} \times 0.25 \mu \mathrm{m}\right.$ Method: $1.0 \mathrm{~mL} \cdot \mathrm{min}^{-1}, 40^{\circ} \mathrm{C}$ to $325^{\circ} \mathrm{C}$ at $10{ }^{\circ} \mathrm{C} \cdot \min ^{-1}$, injector 250 ${ }^{\circ} \mathrm{C}$, detector $250{ }^{\circ} \mathrm{C}$ )

Method C: BetaDex325 column $\left(30 \mathrm{~m} \times 0.32 \mathrm{~mm} \times 0.25 \mu \mathrm{m}\right.$ Method: $1.0 \mathrm{~mL} \cdot \mathrm{min}^{-1}, 90^{\circ} \mathrm{C}$ to $115^{\circ} \mathrm{C}$ at $1{ }^{\circ} \mathrm{C} \cdot \mathrm{min}^{-1}$, injector $250{ }^{\circ} \mathrm{C}$, detector $250{ }^{\circ} \mathrm{C}$ ).

Method D: BetaDex120 column $\left(30 \mathrm{~m} \times 0.32 \mathrm{~mm} \times 0.25 \mu \mathrm{m}\right.$ Method: $1.0 \mathrm{~mL} \cdot \mathrm{min}^{-1}, 70^{\circ} \mathrm{C}$ hold $2 \mathrm{~min}$ then 70 to $150^{\circ} \mathrm{C}$ at 1 ${ }^{\circ} \mathrm{C} \cdot \mathrm{min}^{-1}$, injector $250{ }^{\circ} \mathrm{C}$, detector $250{ }^{\circ} \mathrm{C}$ ).

\subsection{GC-MS}

Method E: Agilent HP-1ms column $\left(30 \mathrm{~m} \times 0.32 \mathrm{~mm} \times 0.25 \mu \mathrm{m}\right.$, Method: $2 \mathrm{~mL} \cdot \mathrm{min}^{-1}, 40{ }^{\circ} \mathrm{C}$ to $300{ }^{\circ} \mathrm{C}$ at $10^{\circ} \mathrm{C} \cdot \mathrm{min}^{-1}$, injector $270{ }^{\circ} \mathrm{C}$, transfer line $320^{\circ} \mathrm{C}$, quadrupole $150{ }^{\circ} \mathrm{C}$, ion source $230{ }^{\circ} \mathrm{C}$ ).

\subsection{List of analyte retention times}

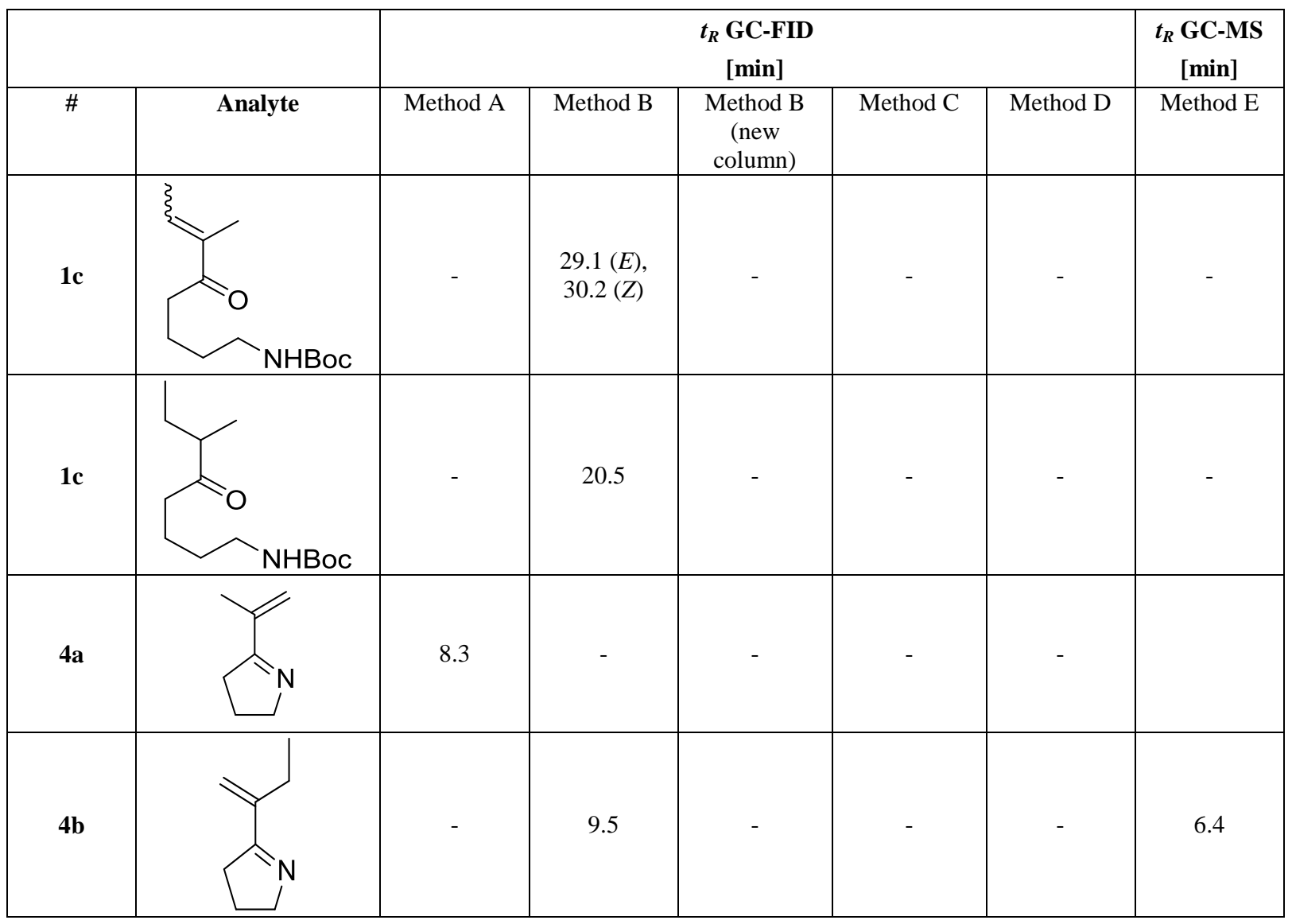




\begin{tabular}{|c|c|c|c|c|c|c|}
\hline $4 c$ & - & - & - & - & - & 7.5 \\
\hline $4 d$ & 9.6 & - & - & - & - & 7.2 \\
\hline $5 a$ & 9.5 & - & - & - & - & 7.0 \\
\hline $5 b$ & - & 13.4 & - & - & - & 8.2 \\
\hline $5 c$ & - & 15.2 & - & - & - & 9.3 \\
\hline $5 d$ & 10.4 & - & - & - & - & 8.7 \\
\hline $6 a$ & - & - & - & - & - & 8.2 \\
\hline $6 \mathrm{~b}$ & - & - & - & - & - & 9.3 \\
\hline $6 c$ & - & - & - & - & - & 10.3 \\
\hline 6d & - & - & - & - & - & 9.9 \\
\hline
\end{tabular}




\begin{tabular}{|c|c|c|c|c|c|c|}
\hline $13 a$ & 7.8 & - & - & - & - & - \\
\hline $13 b=13 c$ & - & 9.1 & - & - & - & 5.9 \\
\hline 13d & 8.8 & - & - & - & - & 6.0 \\
\hline $14 a$ & 9.0 & - & - & - & - & 6.3 \\
\hline $14 \mathrm{~b}=14 \mathrm{c}$ & - & 12.1 & 13.7 & - & - & 7.5 \\
\hline $14 d$ & $9.8^{\mathrm{a}}$ & - & - & - & - & $7.8^{\mathrm{a}}$ \\
\hline $15 \mathrm{a}$ & - & - & - & - & - & $8.2^{\mathrm{a}}$ \\
\hline $15 b=15 c$ & - & - & - & - & - & 9.0 \\
\hline 15d & - & - & - & - & - & $9.0^{\mathrm{a}}$ \\
\hline $16 b=16 c$ & - & - & - & - & - & 6.3 \\
\hline
\end{tabular}




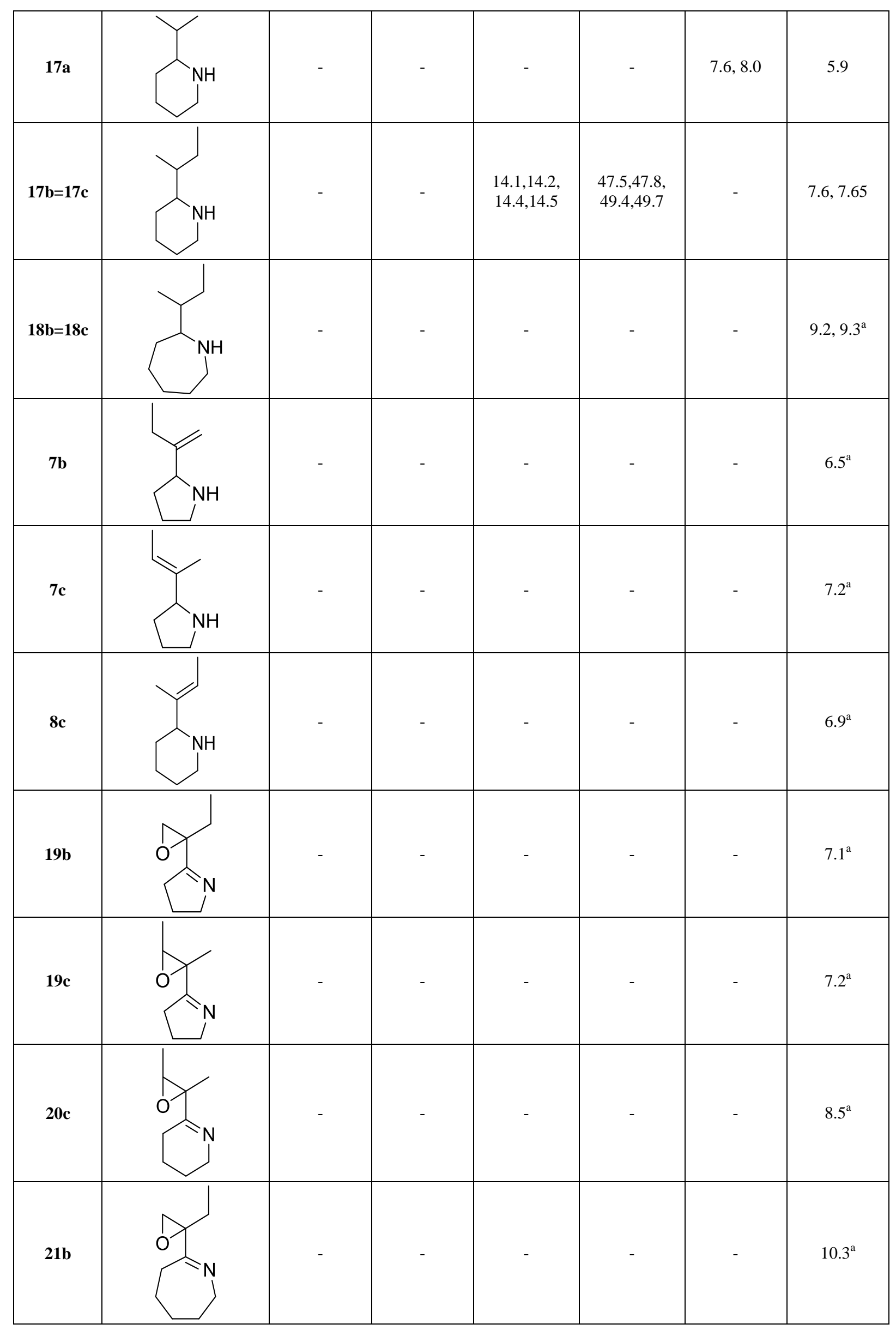




\begin{tabular}{|l|l|l|l|l|l|l|l|}
\hline $21 c$ & - & - & - & - & - & $10.1^{\mathrm{a}}$ \\
\hline
\end{tabular}

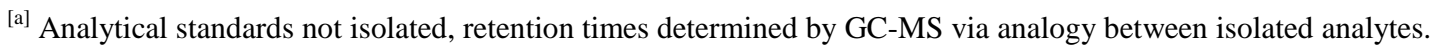




\section{Biotransformations}

Analytical biotransformations were performed using IRED (CFE), ERED (purified or CFE) and CDX-GDH-901 for cofactor recycling. Unless stated otherwise all reactions were at a total volume of $200 \mu \mathrm{L}$ in 96 -well assay plates and shaken at 900 rpm and $25^{\circ} \mathrm{C}$ before being quenched and basified with $5 \mathrm{M} \mathrm{NaOH}$ (aq) $\left(200 \mu \mathrm{L} \cdot \mathrm{mL}^{-1}\right.$ of reaction) and extracted into cyclohexane ( $2 \mathrm{x}$ reaction volume). The organic fractions were combined and analysed by GC-FID or GC-MS

\subsection{Expanded data sets}

\subsubsection{ERED catalysed $\mathrm{C}=\mathrm{C}$ reduction of cyclic enimines (manuscript Table 1$)$}

Table S1. ERED-catalysed $\mathrm{C}=\mathrm{C}$ reduction of cyclic enimines.

\begin{tabular}{|c|c|c|c|c|c|c|c|c|c|c|c|c|}
\hline & & & & $\sum_{-6 a-d}^{R^{2}}$ & RED & $>_{3-15 a}$ & & & & & & \\
\hline \multicolumn{13}{|c|}{ Conversion [\%] } \\
\hline ERED & $4 a$ & $4 b$ & $4 c$ & $4 d$ & $5 \mathbf{a}$ & $5 b$ & $5 c$ & $5 d^{[c]}$ & $6 a^{[c]}$ & $6 b$ & $6 c$ & $\mathbf{6 d}^{[\mathrm{cc}]}$ \\
\hline OYE2 ${ }^{[\mathrm{a}]}$ & 0 & 0 & $0^{[\mathrm{d}]}$ & 0 & 27 & 0 & 0 & 0 & $>99$ & $0^{[\mathrm{d}]}$ & 87 & 0 \\
\hline OYE3 ${ }^{[\mathrm{a}]}$ & 0 & 0 & $0^{[\mathrm{d}]}$ & 0 & 14 & 0 & 0 & 0 & $>99$ & $<1$ & n.t & 0 \\
\hline TOYE $^{[\mathrm{a}]}$ & 89 & 0 & $0^{[\mathrm{d}]}$ & 0 & $>99$ & 17 & 0 & 0 & $>99$ & $>99$ & $80^{[\mathrm{d}]}$ & 0 \\
\hline PETNR $^{[a]}$ & 35 & 0 & $35(21)^{[\mathrm{d}]}$ & 0 & 77 & 24 & 2 & 0 & $>99$ & $>99$ & $>99$ & 52 \\
\hline $\begin{array}{l}\text { PRO- }^{[\mathrm{b}]} \\
\text { ERED01 }^{2}\end{array}$ & n.t. & n.t. & n.t. & n.t. & n.t. & 19 & n.t. & n.t. & n.t. & n.t. & n.t. & n.t. \\
\hline $\begin{array}{c}\text { PRO- }^{[\mathrm{b}]} \\
\text { ERED02 }\end{array}$ & n.t. & n.t. & n.t. & n.t. & n.t. & 0 & n.t. & n.t. & n.t. & n.t. & n.t. & n.t. \\
\hline $\begin{array}{c}\text { PRO- }^{[\mathrm{bb]}} \\
\text { ERED03 }^{2}\end{array}$ & n.t. & n.t. & n.t. & n.t. & n.t. & 3 & n.t. & n.t. & n.t. & n.t. & n.t. & n.t. \\
\hline $\begin{array}{c}\text { PRO- }^{[\mathrm{b}]} \\
\text { ERED04 }\end{array}$ & n.t. & n.t. & n.t. & n.t. & n.t. & 0 & n.t. & n.t. & n.t. & n.t. & n.t. & n.t. \\
\hline $\begin{array}{c}\text { PRO- }^{[\mathrm{bb]}} \\
\text { ERED05 }^{-{ }^{2}}\end{array}$ & n.t. & n.t. & n.t. & n.t. & n.t. & 6 & n.t. & n.t. & n.t. & n.t. & n.t. & n.t. \\
\hline $\begin{array}{c}\text { PRO- }^{[\mathrm{b}]} \\
\text { ERED06 }^{2}\end{array}$ & n.t. & n.t. & n.t. & n.t. & n.t. & 8 & n.t. & n.t. & n.t. & n.t. & n.t. & n.t. \\
\hline $\begin{array}{c}\text { PRO- }^{[\mathrm{b}]} \\
\text { ERED07 }^{2}\end{array}$ & n.t. & n.t. & n.t. & n.t. & n.t. & 46 & n.t. & n.t. & n.t. & n.t. & n.t. & n.t. \\
\hline $\begin{array}{c}\text { PRO- } \\
\text { ERED08 }\end{array}$ & n.t. & $45(21)^{[\mathrm{d}]}$ & $3^{[\mathrm{d}]}$ & 0 & $>99$ & $>99$ & 33 & 0 & $>99$ & $>99$ & $>99$ & 0 \\
\hline $\begin{array}{c}\text { PRO- }^{[\mathrm{b}]} \\
\text { ERED09 }^{2}\end{array}$ & n.t. & n.t. & n.t. & n.t. & n.t. & 8 & n.t. & n.t. & n.t. & n.t. & n.t. & n.t. \\
\hline $\begin{array}{l}\text { PRO- }^{[\mathrm{b}]} \\
\text { ERED10 }\end{array}$ & n.t. & n.t. & n.t. & n.t. & n.t. & 5 & n.t. & n.t. & n.t. & n.t. & n.t. & n.t. \\
\hline $\begin{array}{c}\text { PRO- } \\
\text { ERED11 }{ }^{[b]}\end{array}$ & n.t. & n.t. & n.t. & n.t. & n.t. & 5 & n.t. & n.t. & n.t. & n.t. & n.t. & n.t. \\
\hline
\end{tabular}




\begin{tabular}{|c|c|c|c|c|c|c|c|c|c|c|c|c|}
\hline $\begin{array}{c}\text { PRO- } \\
\text { ERED12 }\end{array}$ & n.t. & n.t. & n.t. & n.t. & n.t. & 0 & n.t. & n.t. & n.t. & n.t. & n.t. & n.t. \\
\hline $\begin{array}{c}\text { PRO- }^{[\mathrm{b}]} \\
\text { ERED13 }^{-{ }^{2}}\end{array}$ & n.t. & n.t. & n.t. & n.t. & n.t. & 4 & n.t. & n.t. & n.t. & n.t. & n.t. & n.t. \\
\hline $\begin{array}{c}\text { PRO- }^{[\mathrm{b}]} \\
\text { ERED14 }^{-{ }^{2}}\end{array}$ & n.t. & n.t. & n.t. & n.t. & n.t. & 4 & n.t. & n.t. & n.t. & n.t. & n.t. & n.t. \\
\hline $\begin{array}{l}\text { PRO- } \\
\text { ERED15 }\end{array}$ & n.t. & n.t. & n.t. & n.t. & n.t. & 21 & n.t. & n.t. & n.t. & n.t. & n.t. & n.t. \\
\hline $\begin{array}{l}\text { PRO- }^{[\mathrm{b}]} \\
\text { ERED16 }^{-{ }^{2}}\end{array}$ & n.t. & n.t. & n.t. & n.t. & n.t. & 5 & n.t. & n.t. & n.t. & n.t. & n.t. & n.t. \\
\hline $\begin{array}{c}\text { PRO- }^{[\mathrm{b}]} \\
\text { ERED17 }^{-{ }^{2}}\end{array}$ & n.t. & n.t. & n.t. & n.t. & n.t. & 5 & n.t. & n.t. & n.t. & n.t. & n.t. & n.t. \\
\hline $\begin{array}{l}\text { PRO- }^{[\mathrm{b}]} \\
\text { ERED18 }^{-{ }^{2}}\end{array}$ & n.t. & n.t. & n.t. & n.t. & n.t. & 8 & n.t. & n.t. & n.t. & n.t. & n.t. & n.t. \\
\hline $\begin{array}{l}\text { PRO- }^{[\mathrm{b}]} \\
\text { ERED19 }^{-{ }^{2}}\end{array}$ & n.t. & n.t. & n.t. & n.t. & n.t. & 5 & n.t. & n.t. & n.t. & n.t. & n.t. & n.t. \\
\hline $\begin{array}{c}\text { PRO- } \\
\text { ERED20 }\end{array}$ & n.t. & $40^{[\mathrm{d}]}$ & $0^{[\mathrm{d}]}$ & 0 & $>99$ & $>99$ & 0 & 0 & $>99$ & $>99$ & $>99$ & 0 \\
\hline $\begin{array}{l}\text { PRO- }^{[\mathrm{b}]} \\
\text { ERED21 }\end{array}$ & n.t. & n.t. & n.t. & n.t. & n.t. & 6 & n.t. & n.t. & n.t. & n.t. & n.t. & n.t. \\
\hline $\begin{array}{c}\text { PRO- } \\
\text { ERED22 }\end{array}$ & n.t. & n.t. & n.t. & n.t. & n.t. & 6 & n.t. & n.t. & n.t. & n.t. & n.t. & n.t. \\
\hline $\begin{array}{c}\text { PRO- }^{[\mathrm{b}]} \\
\text { ERED23 }^{-{ }^{2}}\end{array}$ & n.t. & n.t. & n.t. & n.t. & n.t. & 18 & n.t. & n.t. & n.t. & n.t. & n.t. & n.t. \\
\hline $\begin{array}{l}\text { PRO- }^{\left[{ }^{b}\right]} \\
\text { ERED24 }\end{array}$ & n.t. & n.t. & n.t. & n.t. & n.t. & 3 & n.t. & n.t. & n.t. & n.t. & n.t. & n.t. \\
\hline $\begin{array}{c}\text { PRO- } \\
\text { ERED25 }\end{array}$ & n.t. & n.t. & n.t. & n.t. & n.t. & 30 & n.t. & n.t. & n.t. & n.t. & n.t. & n.t. \\
\hline $\begin{array}{c}\text { PRO- } \\
\text { ERED26 }\end{array}$ & n.t. & n.t. & n.t. & n.t. & n.t. & 5 & n.t. & n.t. & n.t. & n.t. & n.t. & n.t. \\
\hline $\begin{array}{c}\text { PRO- }^{[\mathrm{b}]} \\
\text { ERED27 }^{-{ }^{2}}\end{array}$ & n.t. & n.t. & n.t. & n.t. & n.t. & 0 & n.t. & n.t. & n.t. & n.t. & n.t. & n.t. \\
\hline $\begin{array}{c}\text { PRO- } \\
\text { ERED28 }^{[\mathrm{b}]}\end{array}$ & n.t. & n.t. & n.t. & n.t. & n.t. & 9 & n.t. & n.t. & n.t. & n.t. & n.t. & n.t. \\
\hline $\begin{array}{c}\text { PRO- }^{[\mathrm{b}]} \\
\text { ERED29 }^{-{ }^{2}}\end{array}$ & n.t. & n.t. & n.t. & n.t. & n.t. & 12 & n.t. & n.t. & n.t. & n.t. & n.t. & n.t. \\
\hline $\begin{array}{l}\text { PRO- } \\
\text { ERED30 }\end{array}$ & n.t. & n.t. & n.t. & n.t. & n.t. & 3 & n.t. & n.t. & n.t. & n.t. & n.t. & n.t. \\
\hline $\begin{array}{l}\text { PRO- }^{[\mathrm{b}]} \\
\text { ERED31 }^{-{ }^{2}}\end{array}$ & n.t. & n.t. & n.t. & n.t. & n.t. & 4 & n.t. & n.t. & n.t. & n.t. & n.t. & n.t. \\
\hline $\begin{array}{c}\text { PRO- }^{[\mathrm{b}]} \\
\text { ERED32 }^{-}\end{array}$ & n.t. & n.t. & n.t. & n.t. & n.t. & 4 & n.t. & n.t. & n.t. & n.t. & n.t. & n.t. \\
\hline $\begin{array}{c}\text { PRO- }^{[\mathrm{b}]} \\
\text { ERED33 }^{-{ }^{2}}\end{array}$ & n.t. & n.t. & n.t. & n.t. & n.t. & 7 & n.t. & n.t. & n.t. & n.t. & n.t. & n.t. \\
\hline $\begin{array}{c}\text { PRO- } \\
\text { ERED34 }^{[\mathrm{b}]}\end{array}$ & n.t. & n.t. & n.t. & n.t. & n.t. & 4 & n.t. & n.t. & n.t. & n.t. & n.t. & n.t. \\
\hline
\end{tabular}




\begin{tabular}{|c|c|c|c|c|c|c|c|c|c|c|c|c|}
\hline $\begin{array}{c}\text { PRO- } \\
\text { ERED35 }\end{array}$ & n.t. & n.t. & n.t. & n.t. & n.t. & 4 & n.t. & n.t. & n.t. & n.t. & n.t. & n.t. \\
\hline $\begin{array}{c}\text { PRO- } \\
\text { ERED36 }\end{array}$ & n.t. & n.t. & n.t. & n.t. & n.t. & 16 & n.t. & n.t. & n.t. & n.t. & n.t. & n.t. \\
\hline $\begin{array}{c}\text { PRO- } \\
\text { ERED37 }^{[b]}\end{array}$ & n.t. & n.t. & n.t. & n.t. & n.t. & 19 & n.t. & n.t. & n.t. & n.t. & n.t. & n.t. \\
\hline $\begin{array}{c}\text { PRO- } \\
\text { ERED38 }\end{array}$ & n.t. & n.t. & n.t. & n.t. & n.t. & 9 & n.t. & n.t. & n.t. & n.t. & n.t. & n.t. \\
\hline $\begin{array}{c}\text { PRO- } \\
\text { ERED39 }^{[b]}\end{array}$ & n.t. & n.t. & n.t. & n.t. & n.t. & 0 & n.t. & n.t. & n.t. & n.t. & n.t. & n.t. \\
\hline $\begin{array}{l}\text { PRO- }^{[\mathrm{b}]} \\
\text { ERED40 }\end{array}$ & n.t. & n.t. & n.t. & n.t. & n.t. & 50 & n.t. & n.t. & n.t. & n.t. & n.t. & n.t. \\
\hline $\begin{array}{c}\text { PRO- } \\
\text { ERED41 }^{[b]}\end{array}$ & n.t. & n.t. & n.t. & n.t. & n.t. & 4 & n.t. & n.t. & n.t. & n.t. & n.t. & n.t. \\
\hline $\begin{array}{c}\text { JM- }^{[\mathrm{b}]} \\
\text { ENE101 }^{{ }^{2}}\end{array}$ & n.t. & $5^{[\mathrm{d}]}$ & $6^{[\mathrm{d}]}$ & 0 & $>99$ & 49 & $\begin{array}{c}89 \\
(>99)^{[\mathrm{d}]}\end{array}$ & 0 & $>99$ & $>99$ & $>99$ & 0 \\
\hline $\begin{array}{c}\text { JM- } \\
\text { ENE102 }\end{array}$ & n.t. & $7^{[\mathrm{d}]}$ & $10^{[\mathrm{d}]}$ & 0 & $>99$ & 44 & $\begin{array}{c}97 \\
(94)^{[\mathrm{d}]}\end{array}$ & 0 & $>99$ & $>99$ & $>99$ & 59 \\
\hline $\begin{array}{c}\text { JM- } \\
\text { ENE103 }^{[b]}\end{array}$ & n.t. & $10^{[\mathrm{d}]}$ & $0^{[\mathrm{d}]}$ & 0 & $>99$ & 64 & 11 & 0 & $>99$ & $>99$ & $95^{[\mathrm{d}]}$ & 0 \\
\hline $\begin{array}{c}\text { JM- }^{[\mathrm{b}]} \\
\text { ENE104 }\end{array}$ & n.t. & $7^{[\mathrm{d}]}$ & $0^{[\mathrm{d}]}$ & 0 & $>99$ & 0 & 1 & 0 & $>99$ & 2 & 82 & 0 \\
\hline$N t \mathrm{DBR}^{[\mathrm{a}]}$ & $>99$ & 84 & 11 & 24 & $>99$ & $>99$ & $>99$ & 18 & $>99$ & $>99$ & $>99$ & $>99$ \\
\hline
\end{tabular}

Conversion was determined by GC analysis after 24 h. n.t. $=$ not tested. Reaction conditions: $5 \mathrm{mM}$ enimine, ${ }^{\text {[a] }} 2 \mathrm{mg} \cdot \mathrm{mL}^{-1}$ purified ERED or ${ }^{[\mathrm{b}]} 5 \mathrm{mg} \cdot \mathrm{mL}^{-1}$ ERED CFE, $0.1 \mathrm{mg} \cdot \mathrm{mL}^{-1} \mathrm{GDH}, 0.1 \mathrm{mM} \mathrm{NADP}{ }^{+}, 50 \mathrm{mM}$ glucose, $5 \% v / v$ DMSO, $100 \mathrm{mM}$ potassium phosphate buffer $\mathrm{pH}$ 7.0. ${ }^{[\mathrm{c}]}$ Product determined by analogy based on the GC-MS spectrum. [d] $\alpha, \beta$-epoxy imine observed, reported conversion in brackets refer to the addition of $0.1 \mathrm{mg} \cdot \mathrm{mL}^{-1}$ catalase. 


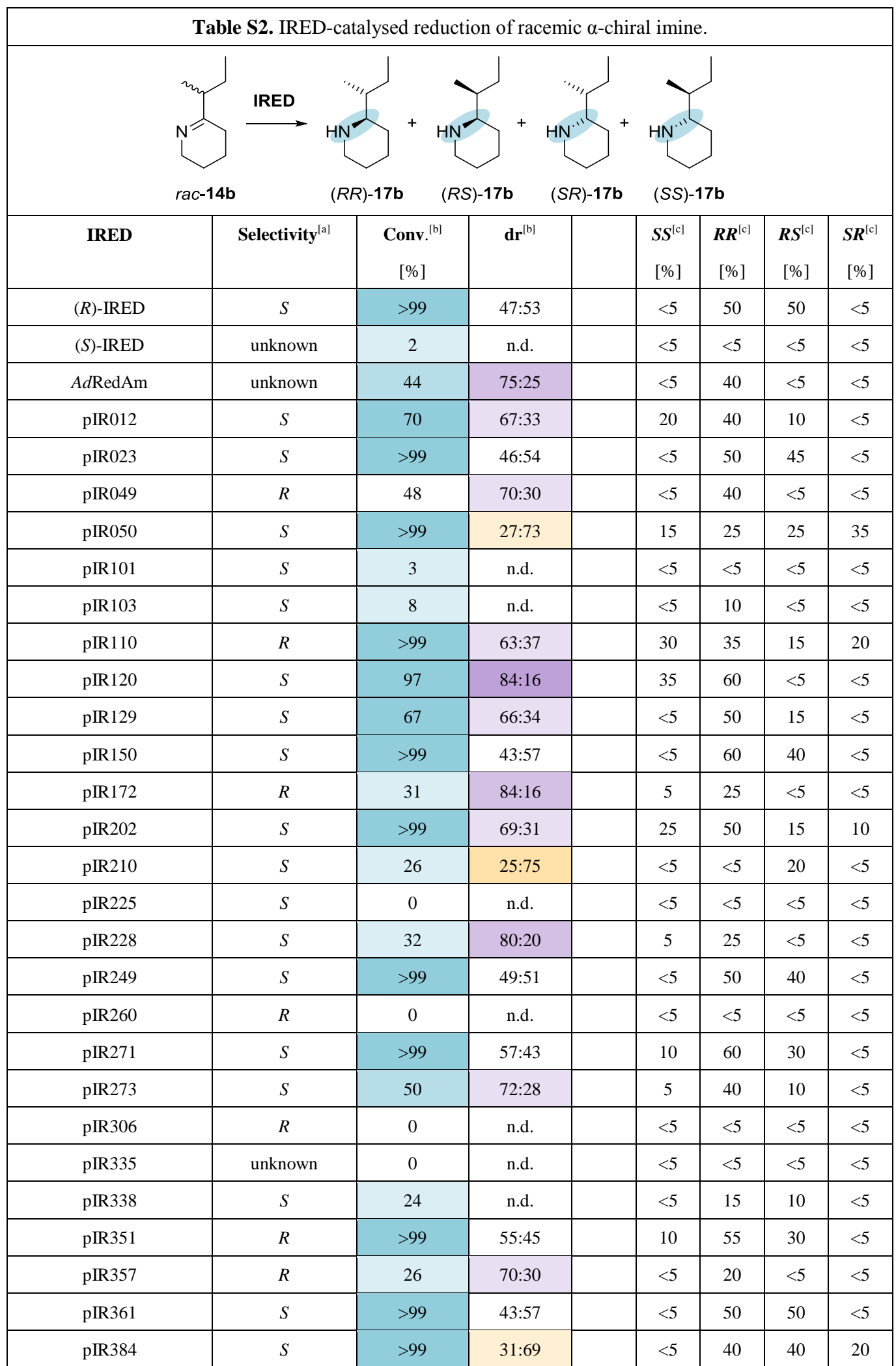

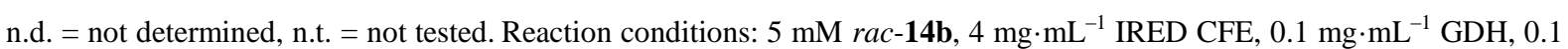
$\mathrm{mM} \mathrm{NADP}{ }^{+}, 10 \mathrm{mM}$ glucose, $5 \% \mathrm{v} / \mathrm{v}$ DMSO, $100 \mathrm{mM}$ potassium phosphate buffer $\mathrm{pH}$ 7.0. ${ }^{[\mathrm{a}]}$ Selectivity toward 6-phenyl2,3,4,5-tetrahydropyridine as provided by the supplier, unless stated. ${ }^{[\mathrm{b}]}$ Conversion and $\mathrm{dr}$ were determined after $24 \mathrm{~h}$ by GC-MS. ${ }^{[c]}$ Assignment through analogy to cascade analysis, extracts were spiked with racemic product to ensure assignment, percentages are rounded to the nearest $5 \%$ to account for analytical accuracy. 


\subsubsection{ERED-IRED telescopic synthesis of saturated $N$-heterocycles (manuscript Scheme 3)}

Scheme S2: ERED-IRED telescopic synthesis of saturated $N$-heterocycles
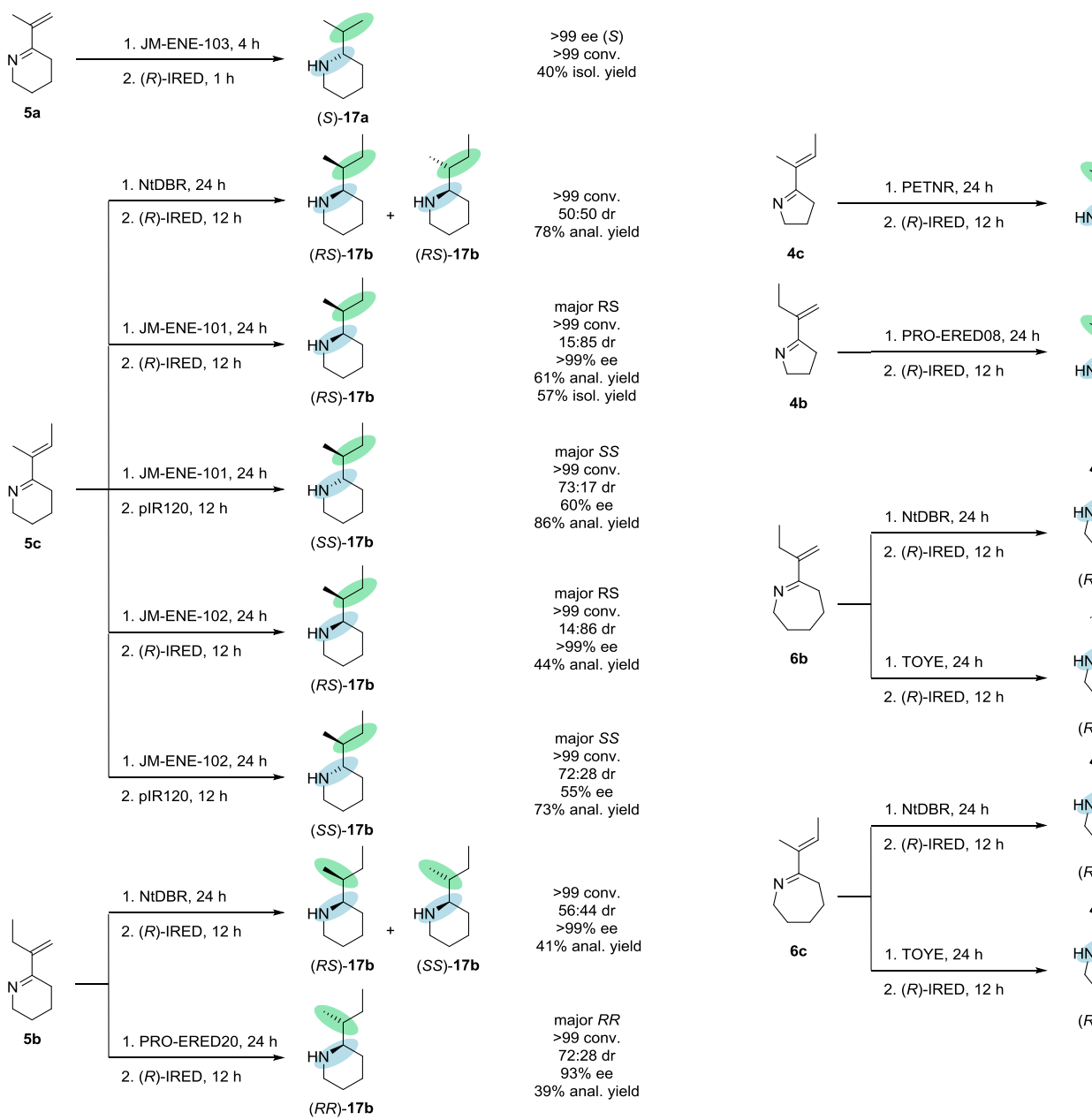

$24 \%$ conv.

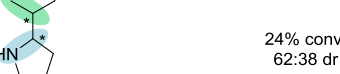

$78 \%$ conv
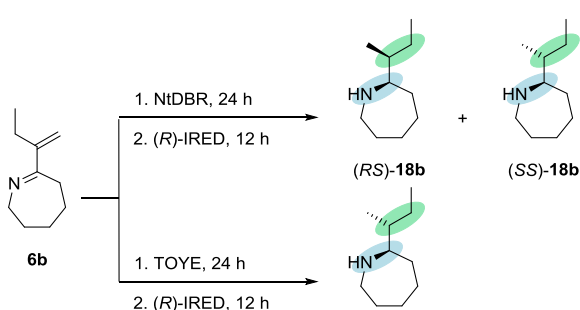

$60 \%$ conv
$47.53 \mathrm{dr}$

$(R S)-18 \mathrm{~b} \quad(S S)-18 \mathrm{~b}$

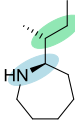

major RR

$55 \%$ con

(RS)-18b
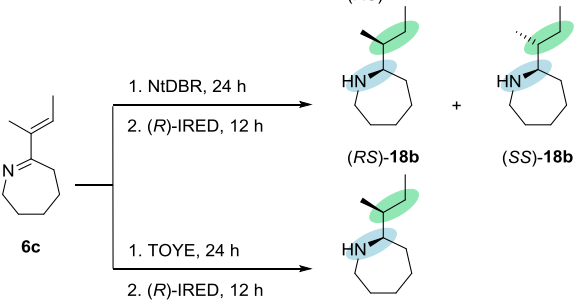

$60 \%$ conv
$48: 52 \mathrm{dr}$

$(S S)-18 \mathrm{~b}$

$(R S)-18 \mathbf{b}$

Reaction conditions: $20 \mathrm{mM}$ enimine, $16 \mathrm{mg} \cdot \mathrm{mL}^{-1}$ ERED and IRED CFE, $0.1 \mathrm{mg} \cdot \mathrm{mL}^{-1}$ catalase, $1 \mathrm{mg} \cdot \mathrm{mL}^{-1} \mathrm{GDH}, 0.1 \mathrm{mM}$ $\mathrm{NADP}^{+}, 100 \mathrm{mM}$ glucose, $5 \% v / v$ DMSO, $100 \mathrm{mM}$ potassium phosphate buffer $\mathrm{pH}$ 7.0. Conversion was determined by GCMS analysis. Reactions were shaken at $900 \mathrm{rpm}$ and $25{ }^{\circ} \mathrm{C}$ for $24 \mathrm{~h}$ before the addition of IRED. Biotransformations were shaken for an additional $12 \mathrm{~h}$ under the same conditions before being quenched with $40 \mu \mathrm{L}$ of $5 \mathrm{M} \mathrm{NaOH}(\mathrm{aq})$ and extracted into cyclohexane $(2 \times 100 \mu \mathrm{L})$. The organic fractions were combined and analysed by GC-MS to determine conversion and diastereoselectivity. Analytical yields were determined via comparison to external calibration. Stereoselectivity determined by chiral GC following derivatisation. The same samples were then derivatised with trifluoroacetic anhydride and triethylamine and compared to literature in order to determine the enantioselectivity of the reaction ${ }^{\mathrm{S} 10}$ (absolute configuration of $\mathbf{1 8 b}$ was assigned by analogy with $\mathbf{1 7 b})$. 

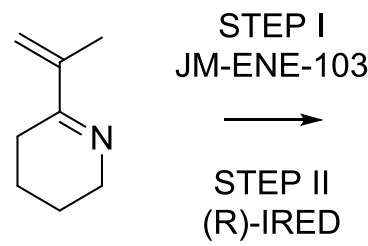<smiles>CC(C)[C@H]1CCCCN1</smiles>

(R)-2-(iso-propyl)piperidine $(R)-17$ a

$52 \mathrm{mg}(220 \mu$ moles $)$ of enimine trifluoroacetate was solubilised in $11 \mathrm{~mL}$ of a solution of $5 \%$ DMSO in phosphate buffer $(\mathrm{pH} 7,100 \mathrm{mM})$ containing $176 \mathrm{mg}$ of JM-ENE-103, $11 \mathrm{mg}$ CDX-GDH, $1 \mathrm{mg}$ catalase, $396 \mathrm{mg}$ glucose, $2 \mathrm{mg}$ NADP $^{+}$. The reaction was shaken in an incubator at $200 \mathrm{rpm}$ and $25{ }^{\circ} \mathrm{C}$ for $4 \mathrm{~h}$ before the addition of $176 \mathrm{mg}(R)$-IRED CFE. Biotransformations were shaken for an additional $1 \mathrm{~h}$ under the same conditions before being basified with $5 \mathrm{M} \mathrm{NaOH} \mathrm{(aq)}$ and extracted into MTBE ( $3 \times 10 \mathrm{~mL})$. Centrifugation was employed to separate the organic fractions which were combined dried filtered and then the filtrate was acidified to yield the amine hydrochloride as a brown solid (14 mg, 40\%). Data is consistent with existing literature. ${ }^{\mathrm{S} 9}$<smiles>CC=C(C)C1=NCCCC1</smiles><smiles>CC[C@H](C)[C@H]1CCCCN1</smiles>

(R)-2-((S)-sec-butyl)piperidine (RS)-17b

$33 \mathrm{mg}(131 \mu$ moles $)$ of enimine trifluoroacetate was solubilised in $6.5 \mathrm{~mL}$ of a solution of $5 \%$ DMSO in phosphate buffer $(\mathrm{pH} 7,100 \mathrm{mM})$ containing $100 \mathrm{mg}$ of JM-ENE-102, $1 \mathrm{mg}$ CDX-GDH, $1 \mathrm{mg}$ catalase, $120 \mathrm{mg}$ glucose. The reaction was shaken in an incubator at $200 \mathrm{rpm}$ and $25^{\circ} \mathrm{C}$ for $16 \mathrm{~h}$ before the addition of $100 \mathrm{mg}(R)$-IRED CFE. Biotransformations were shaken for an additional $2 \mathrm{~h}$ under the same conditions before being basified with $5 \mathrm{M} \mathrm{NaOH}(\mathrm{aq})$ and extracted into MTBE $(3 \times 10 \mathrm{~mL})$. Centrifugation was employed to separate the organic fractions which were combined dried filtered and then the filtrate was acidified to yield the amine hydrochloride as a light yellow solid (13 $\mathrm{mg}, 57 \%)$. 
4.2 GC chromatography

4.2.1 ERED catalysed $\mathrm{C}=\mathrm{C}$ reduction of cyclic enimines

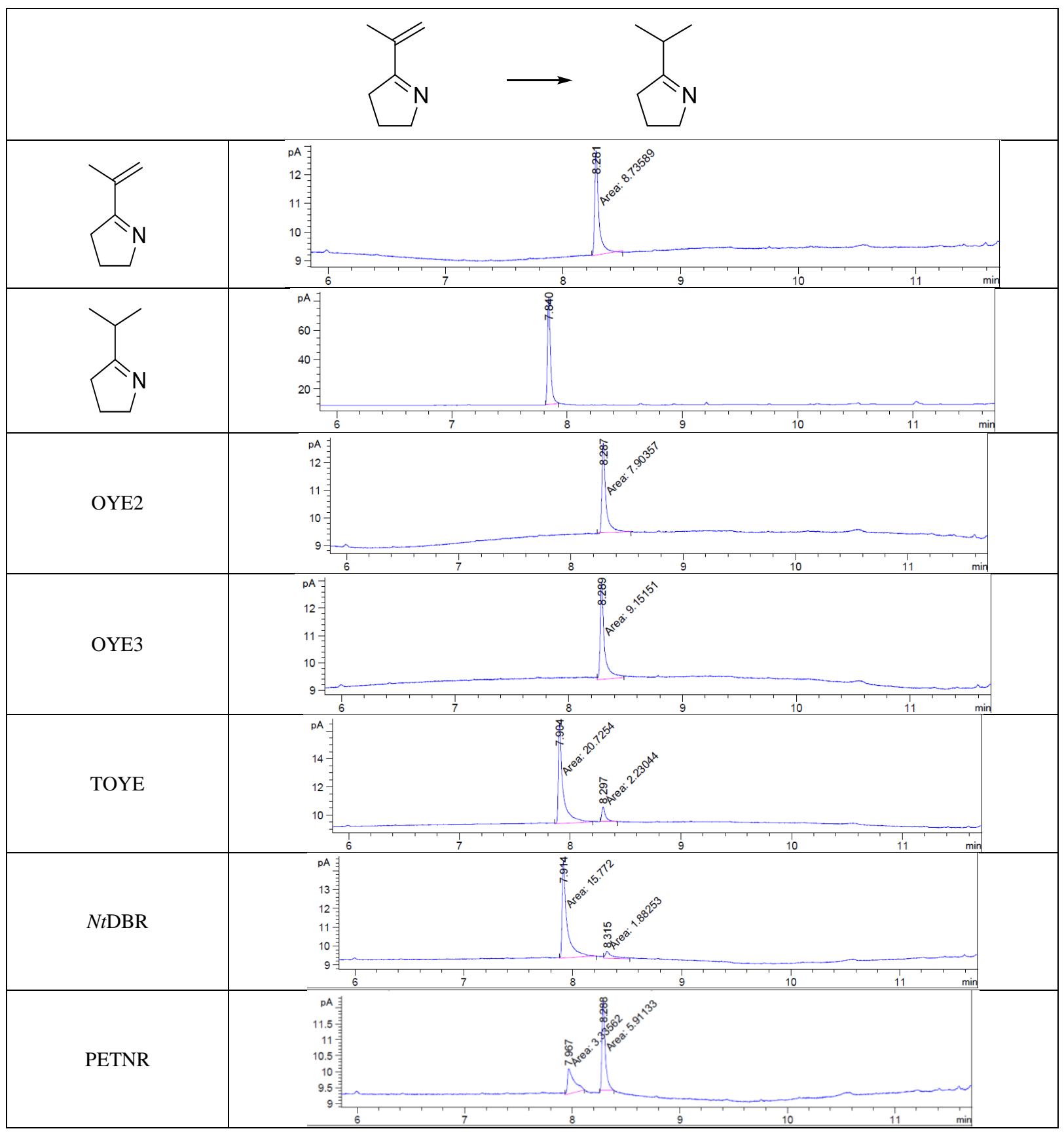




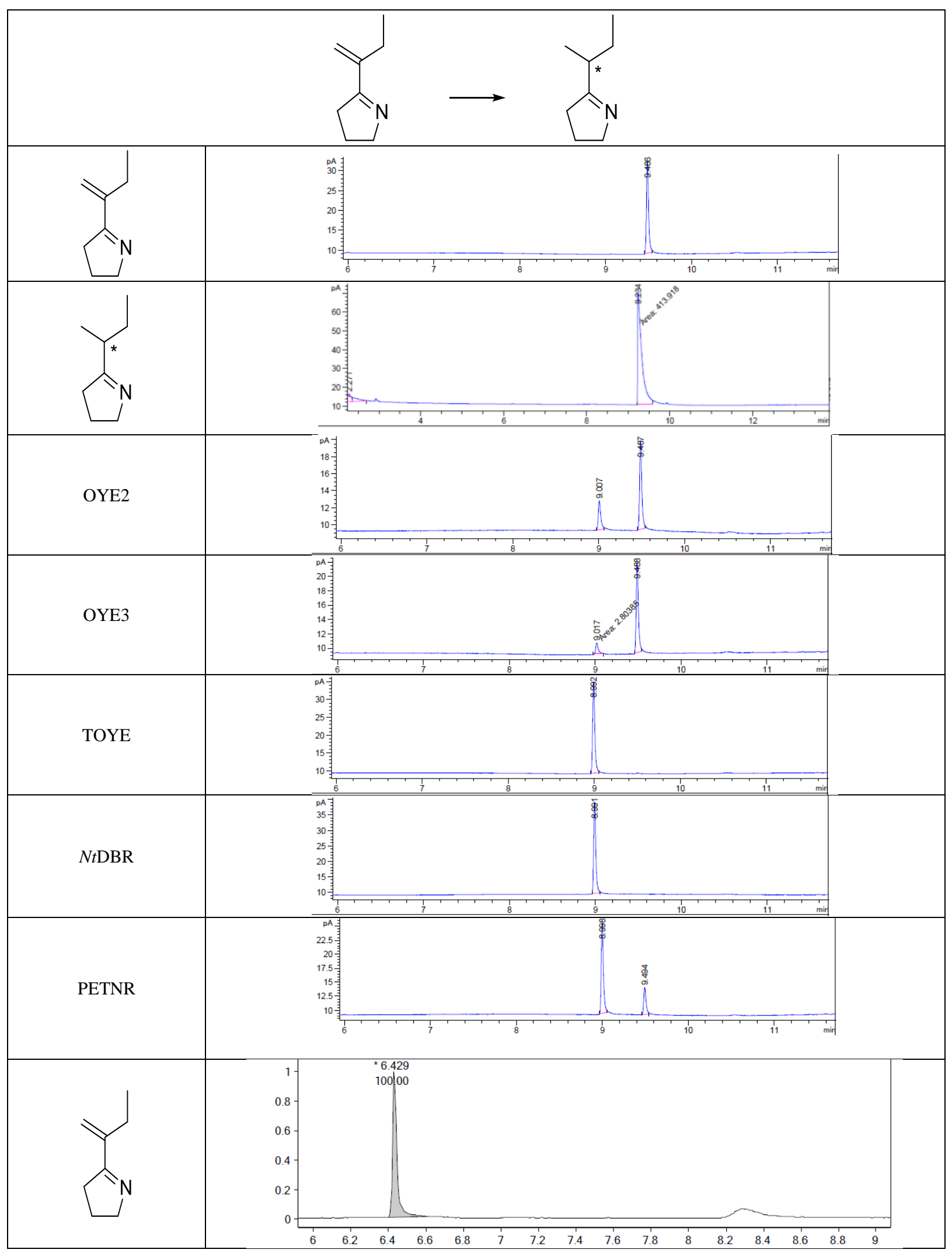




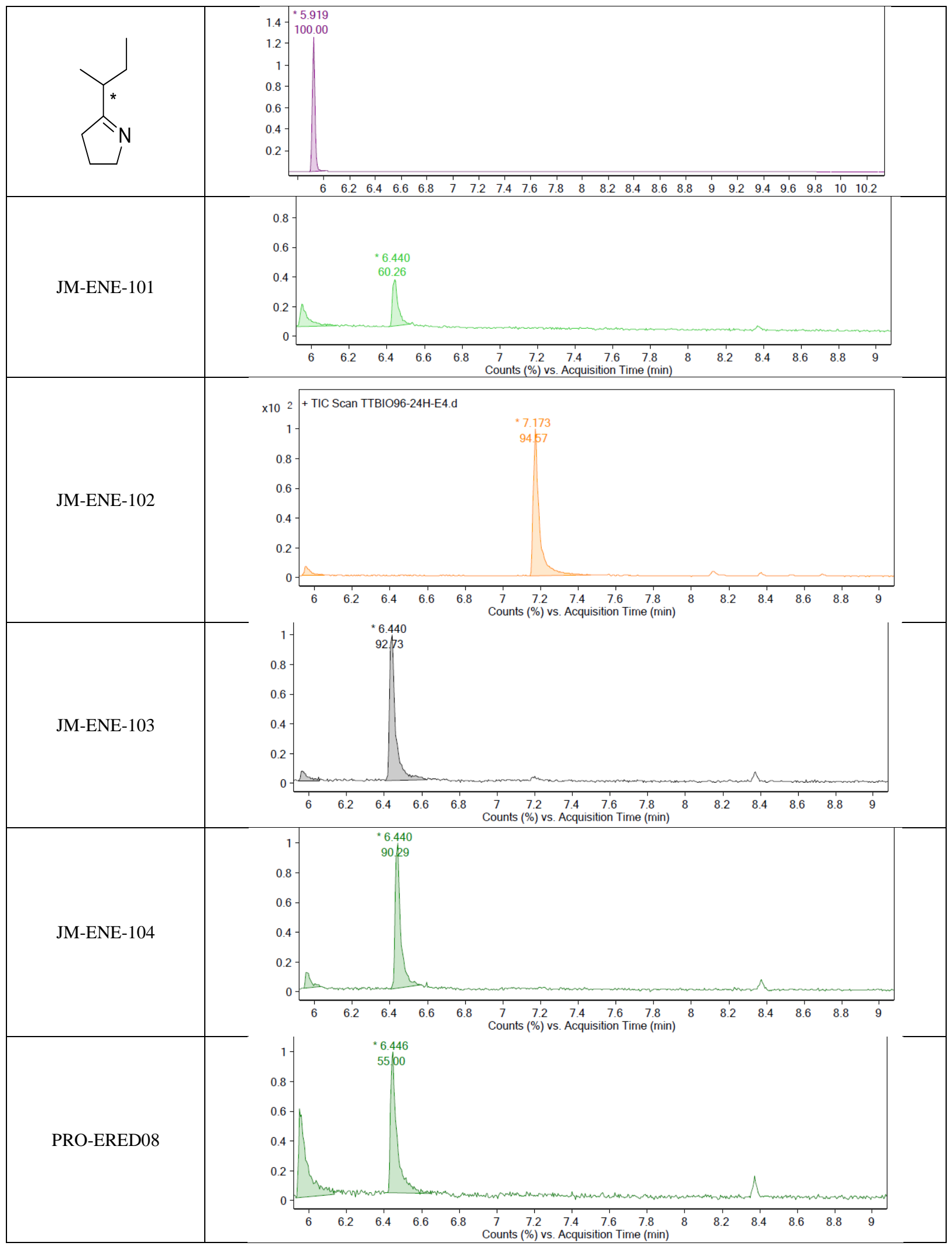



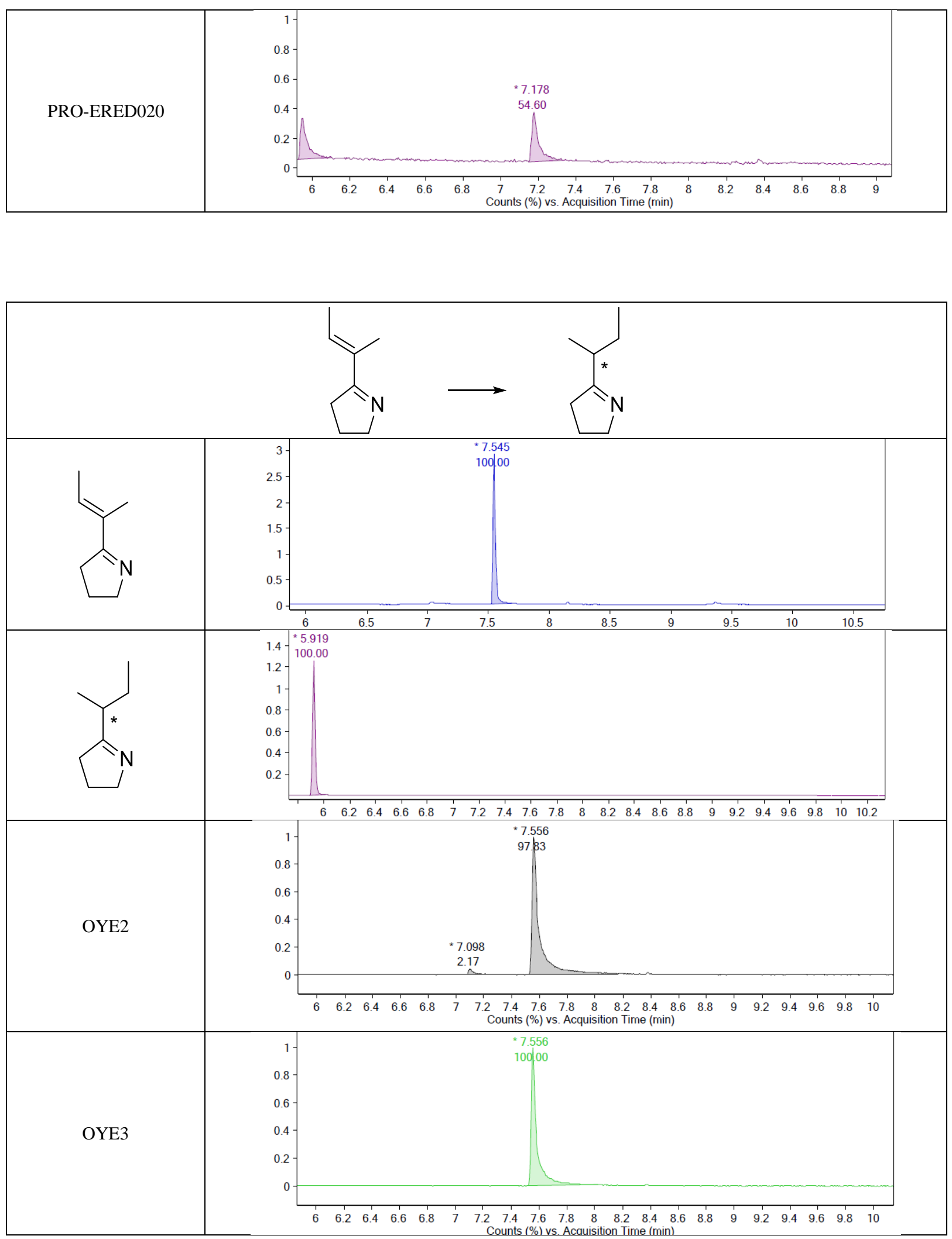


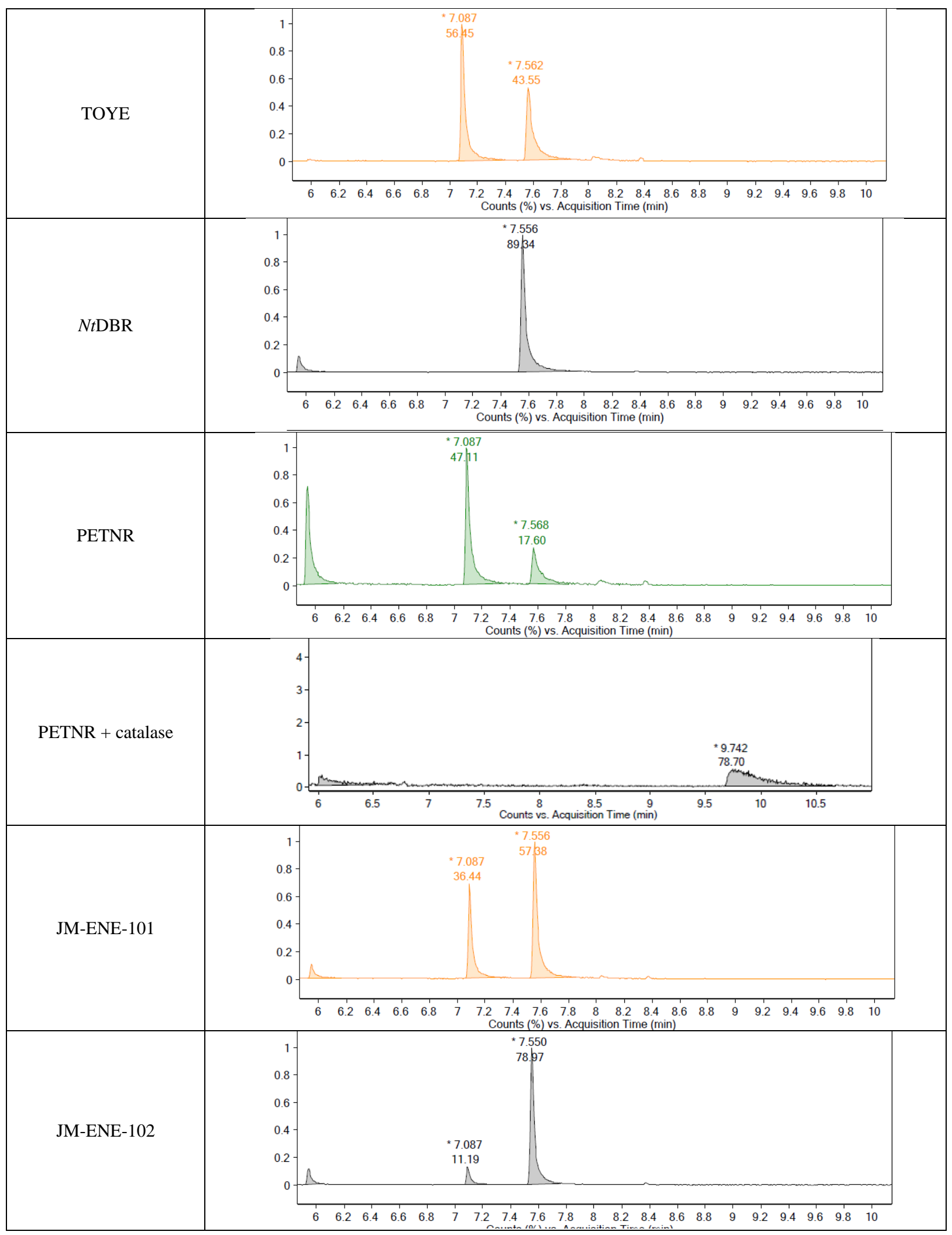



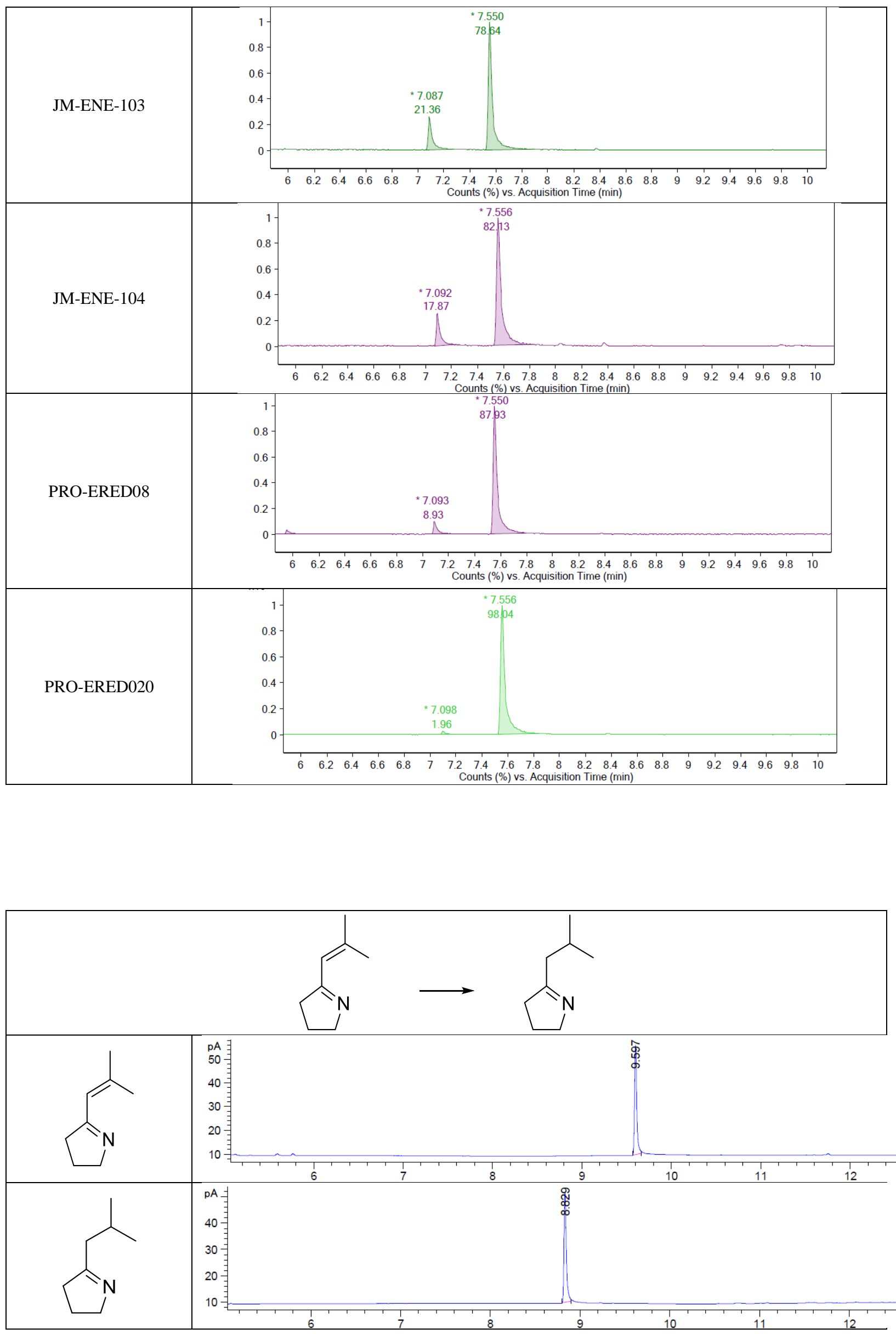


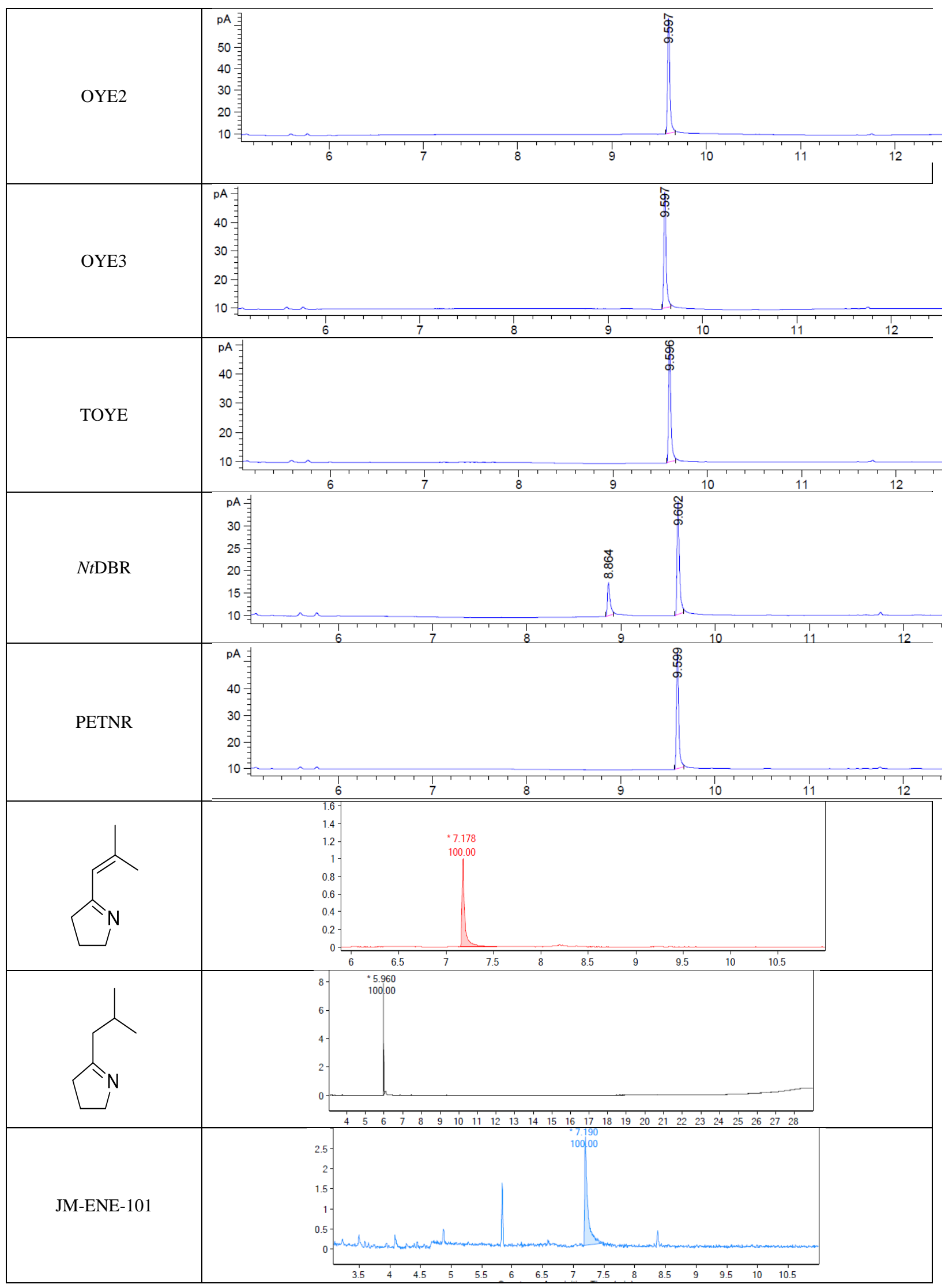



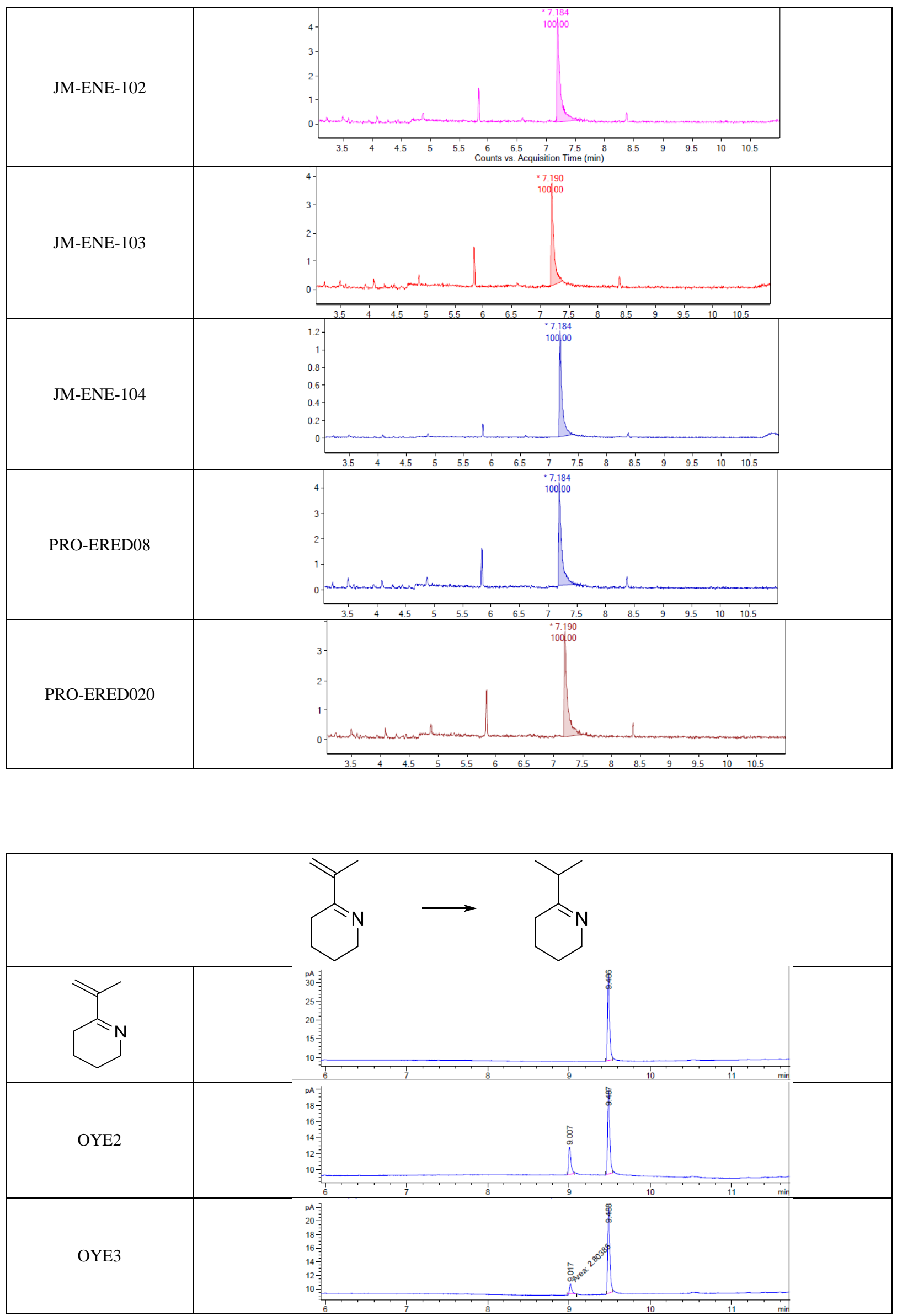


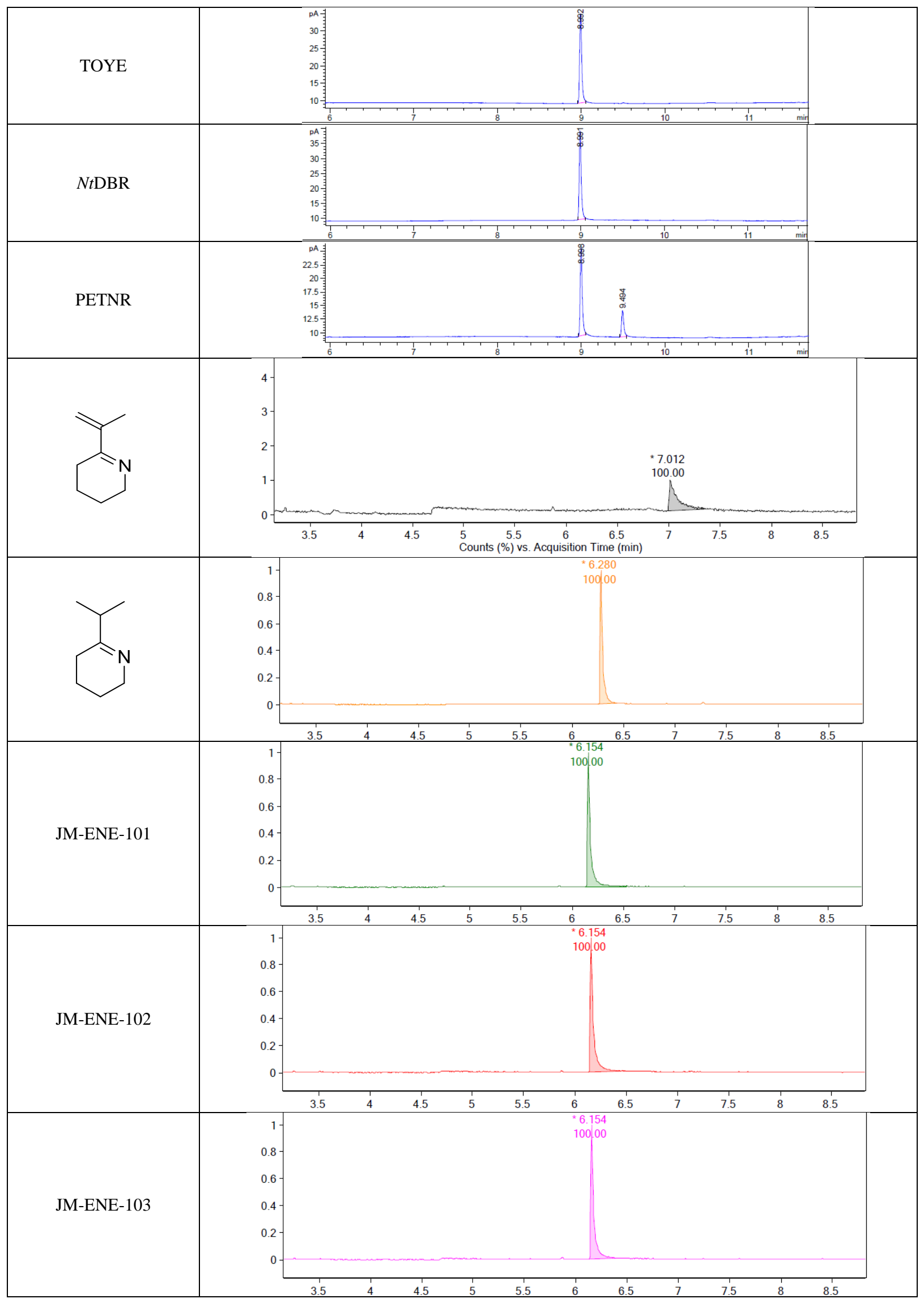



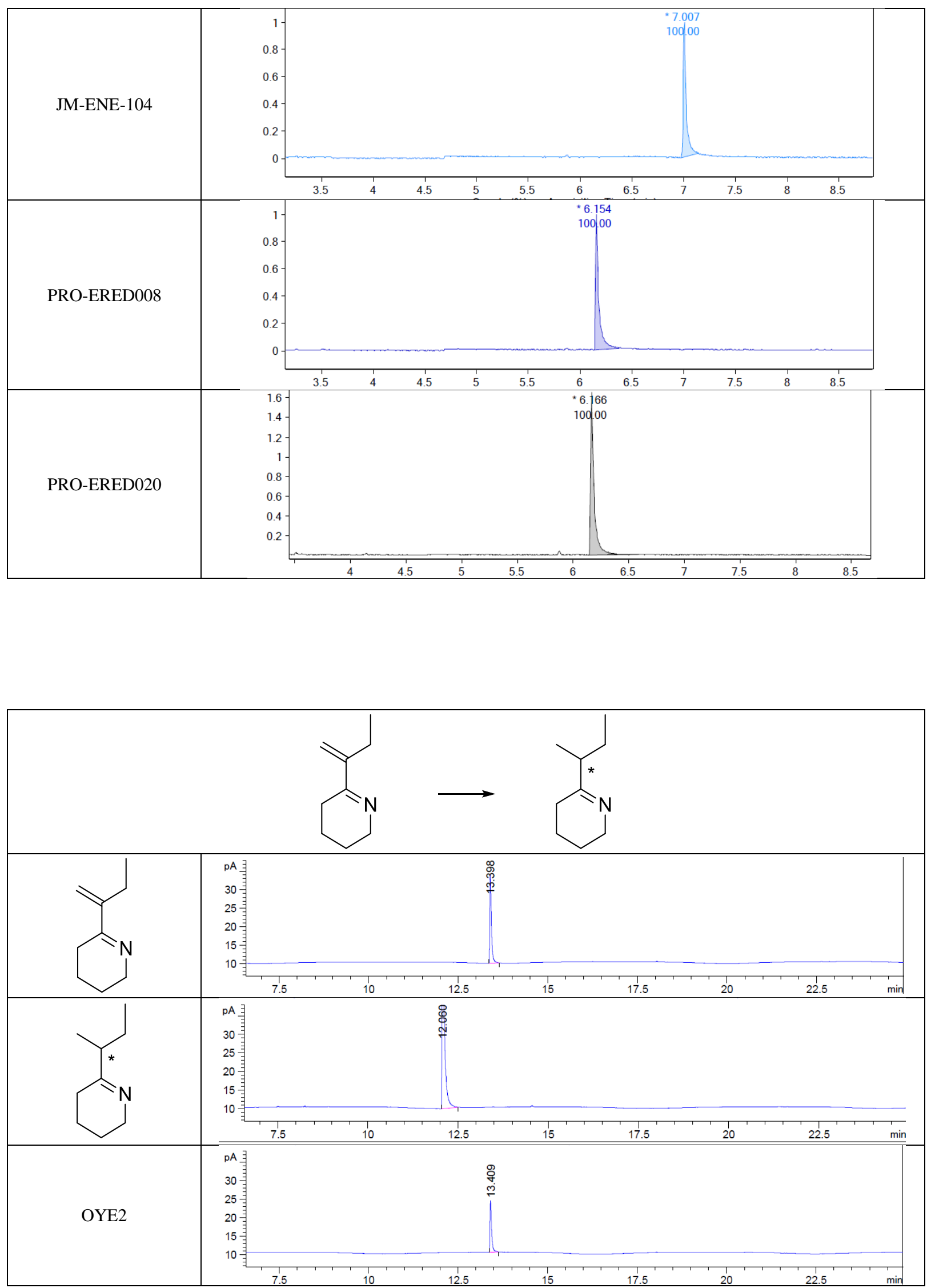


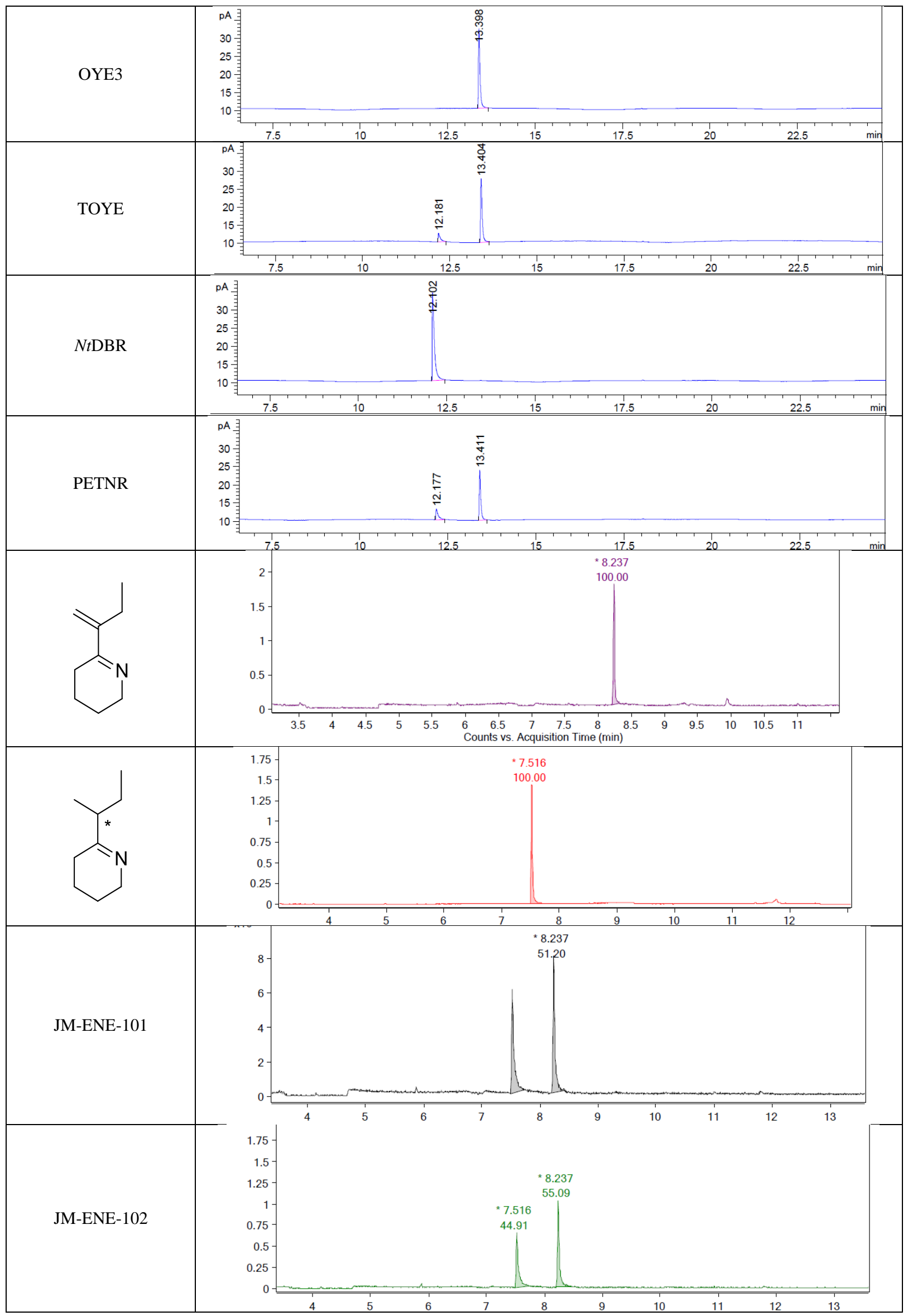




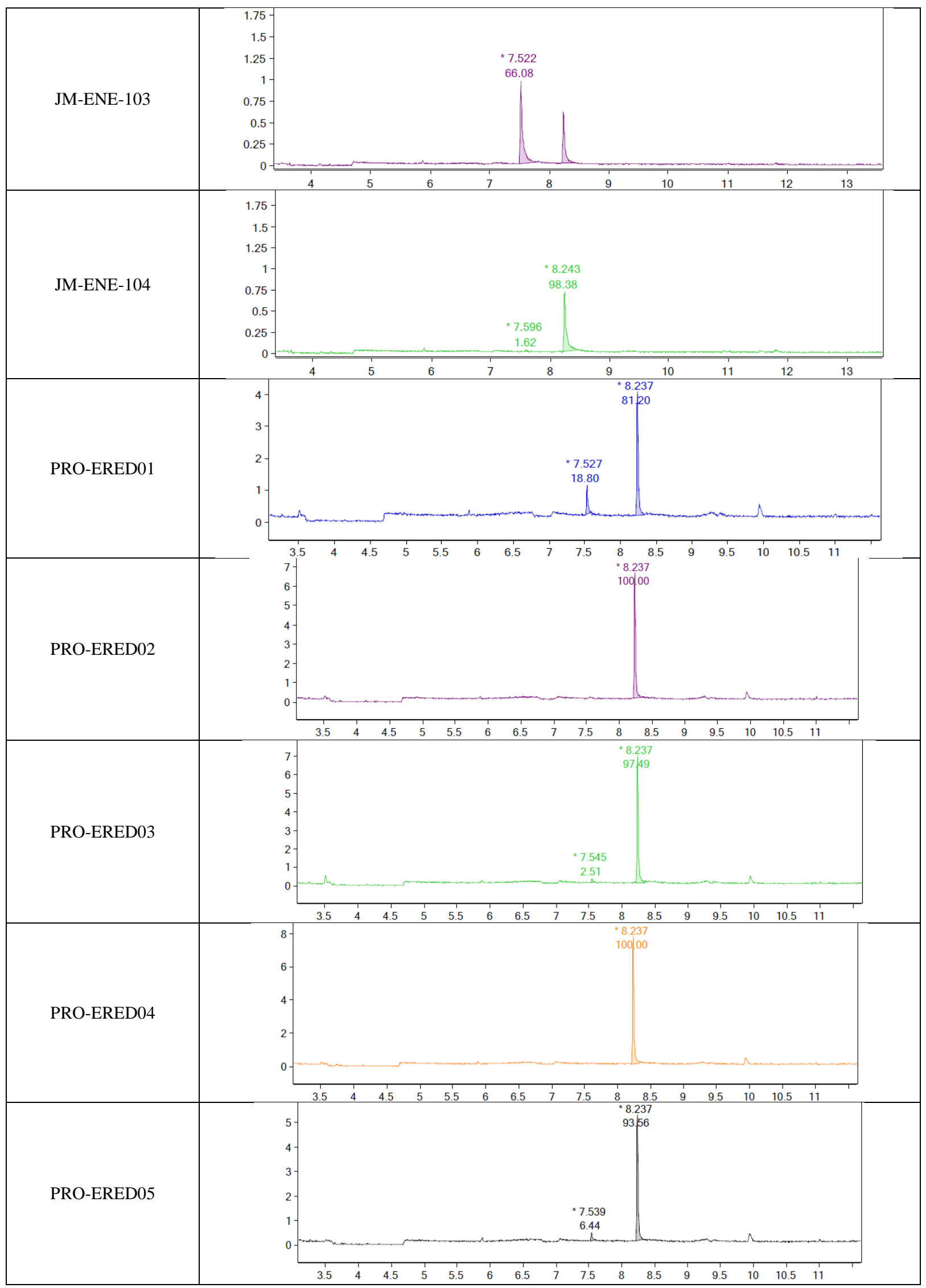




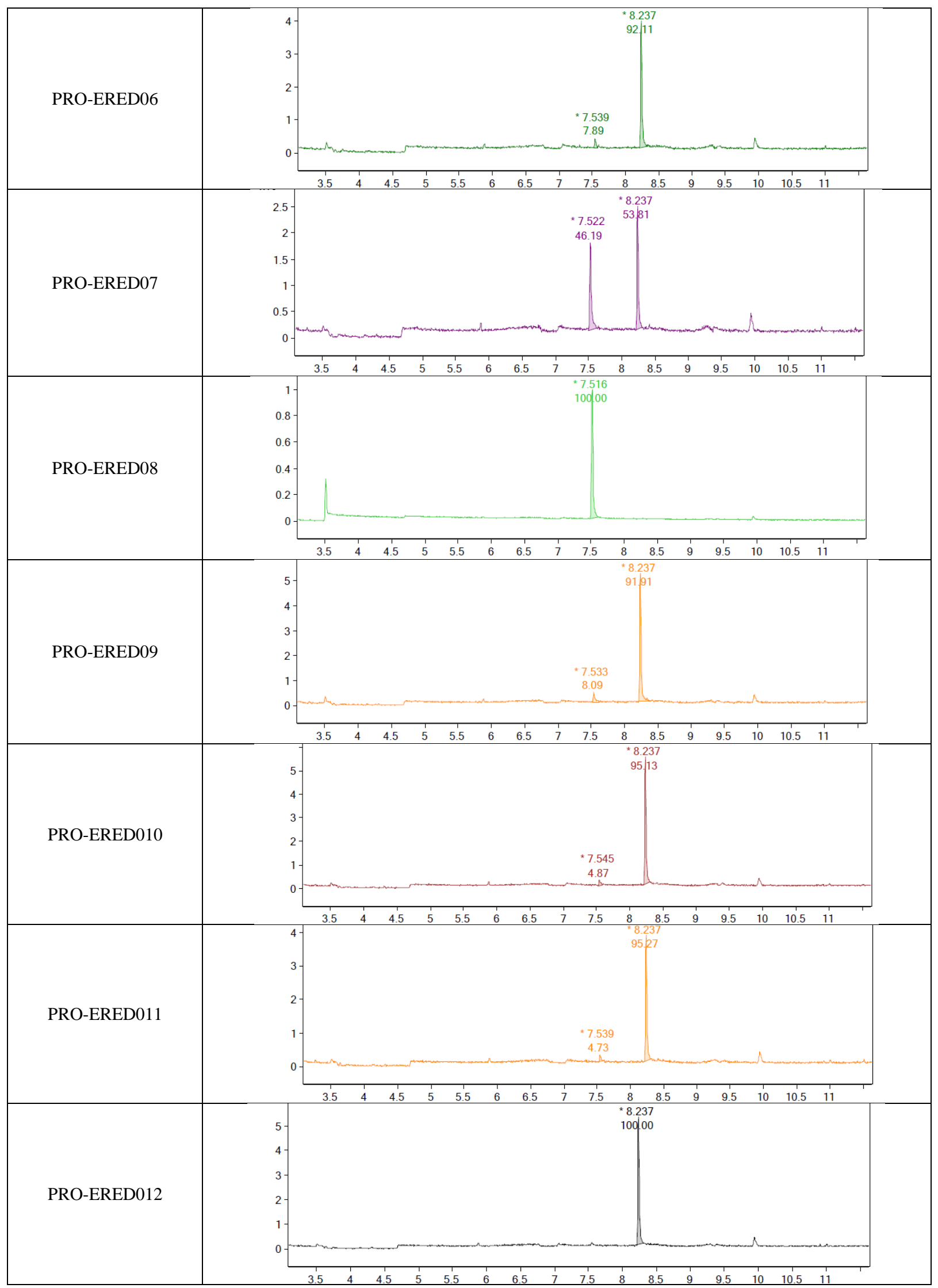




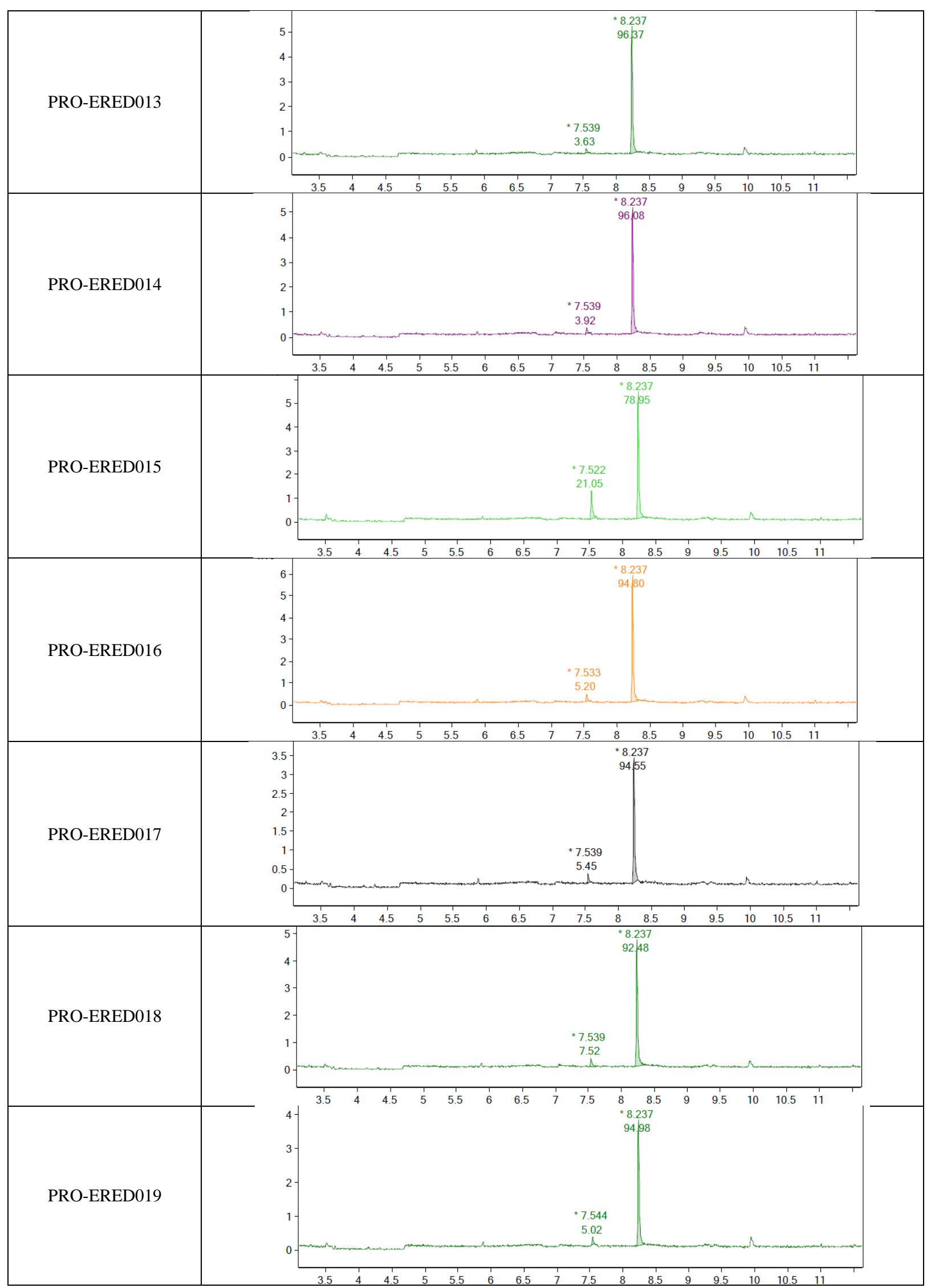




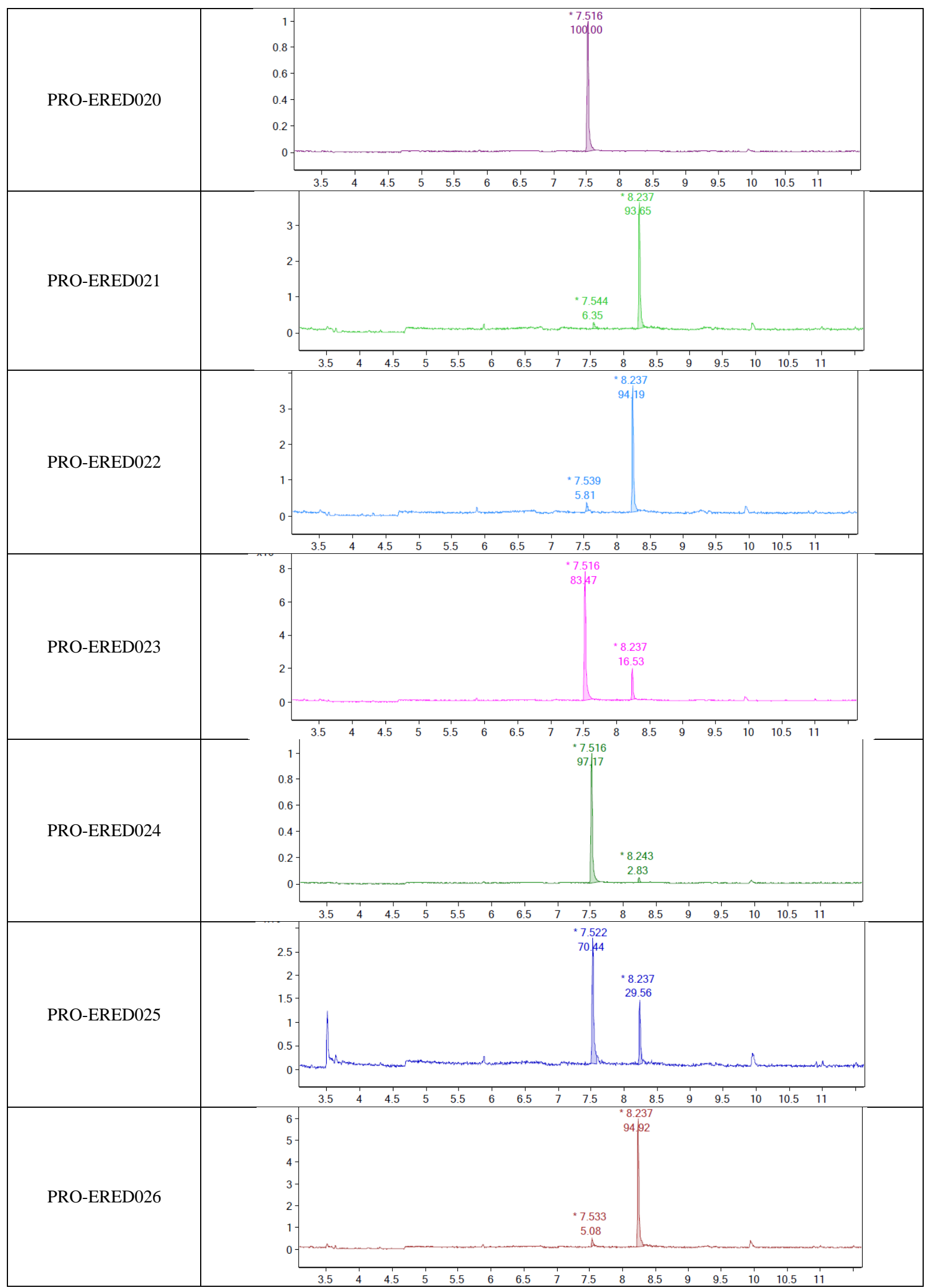




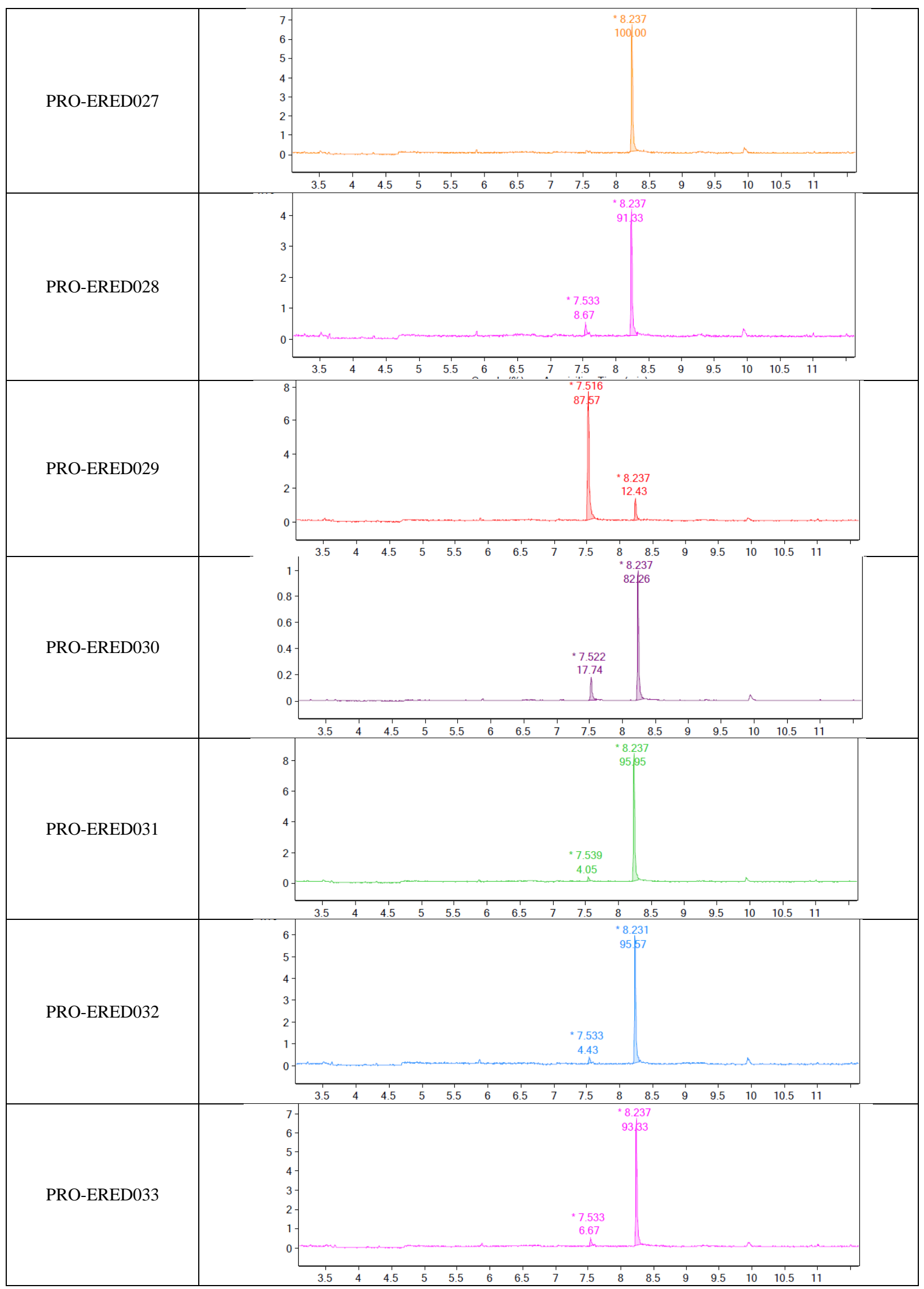




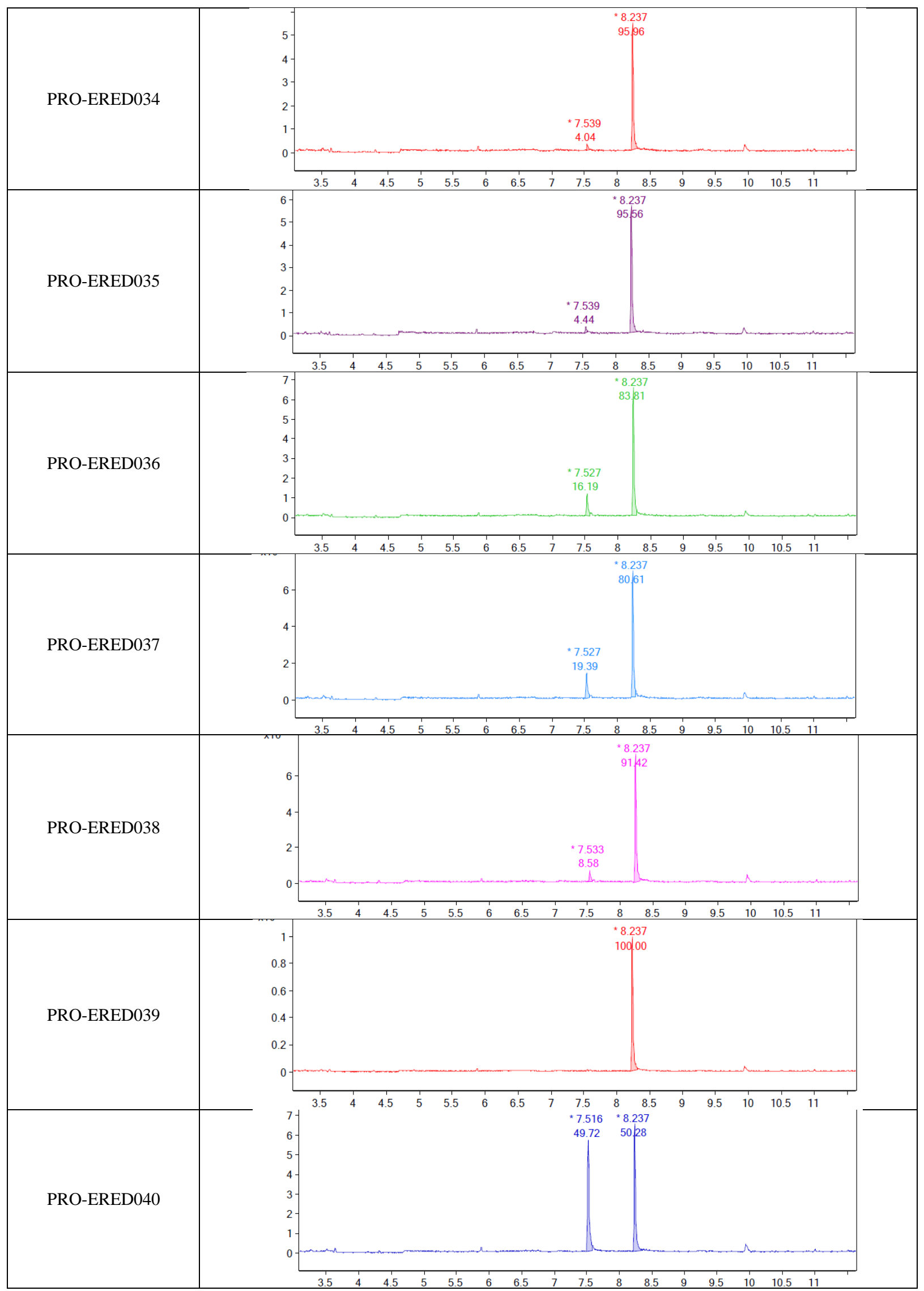



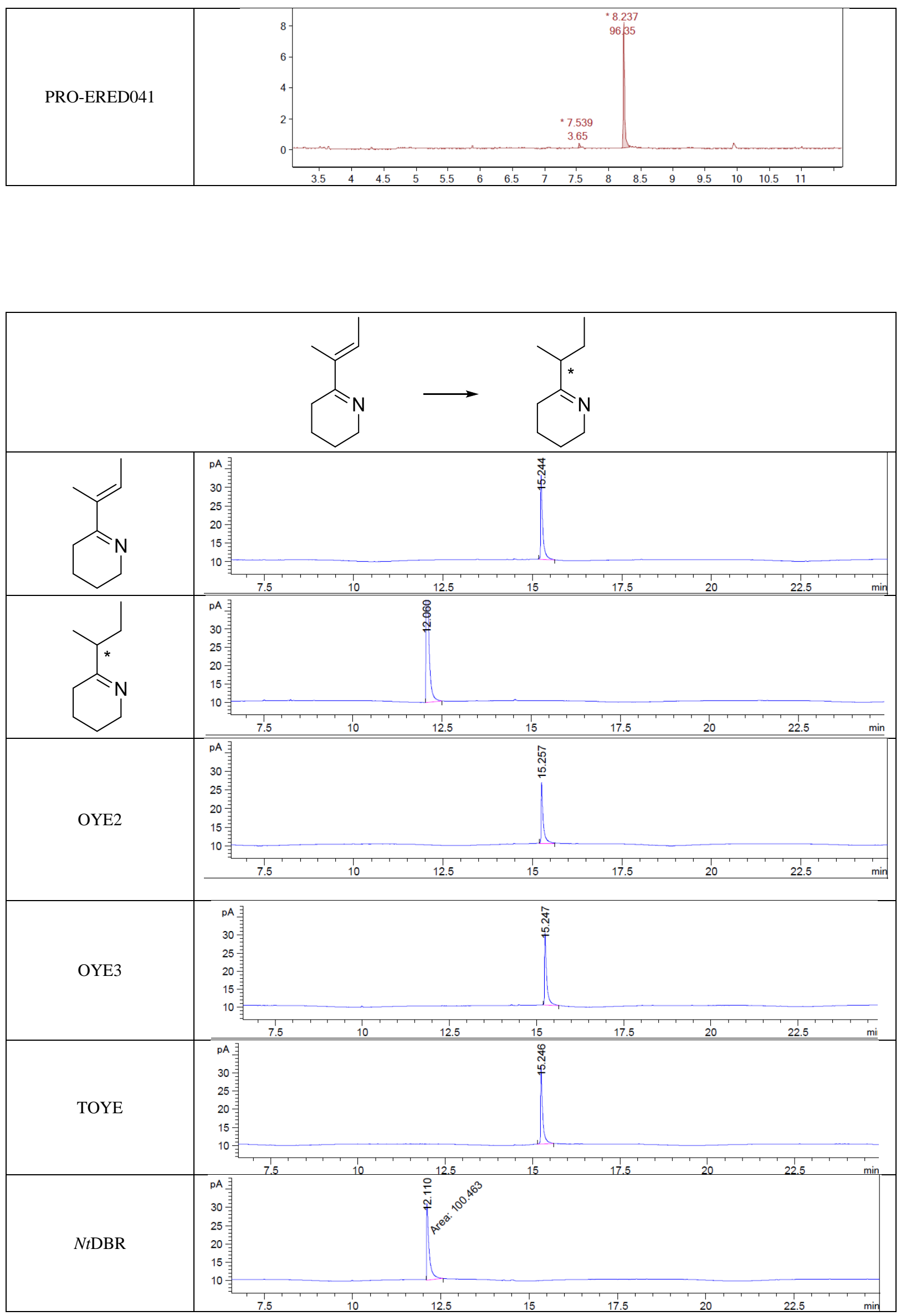


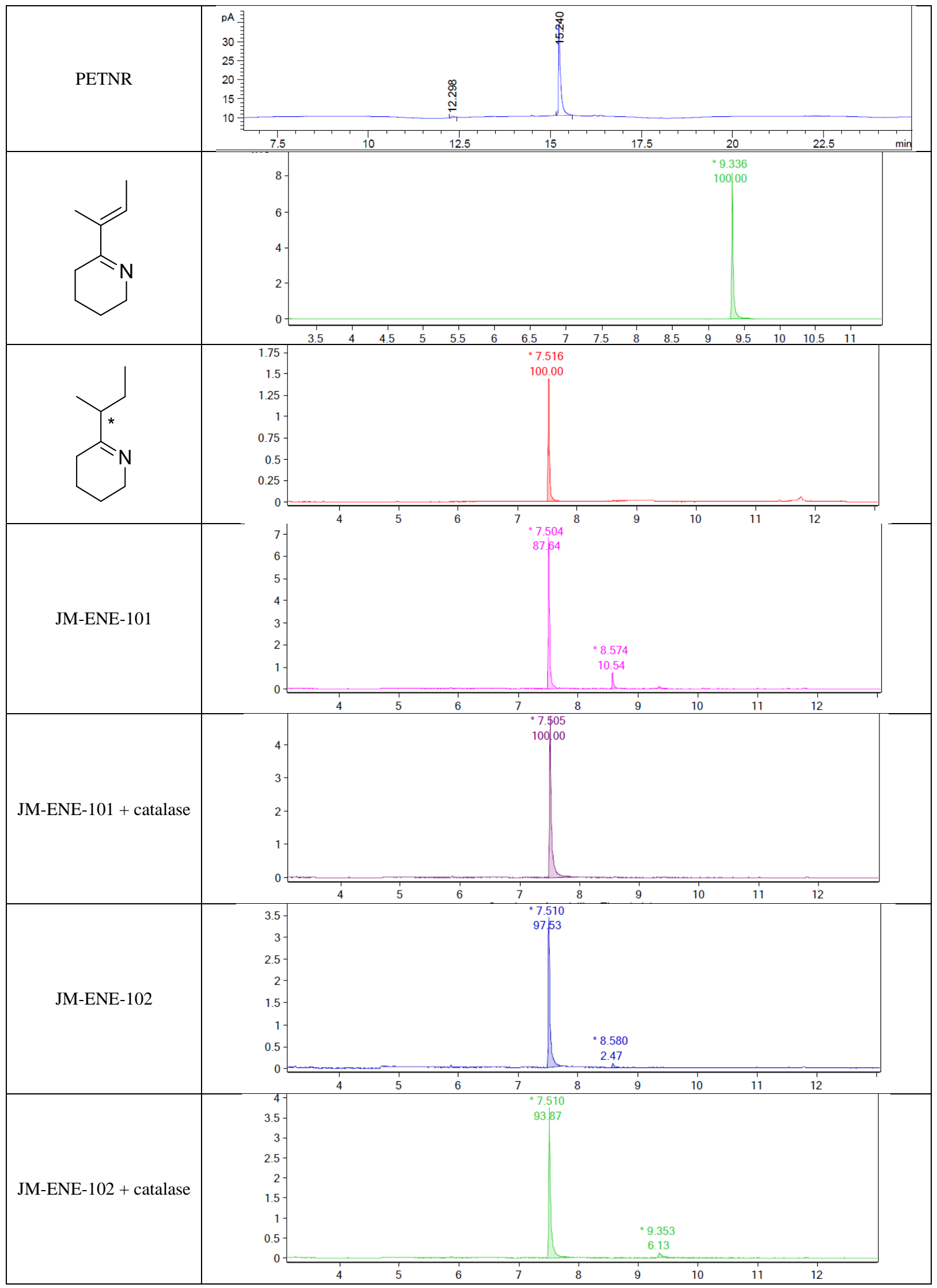



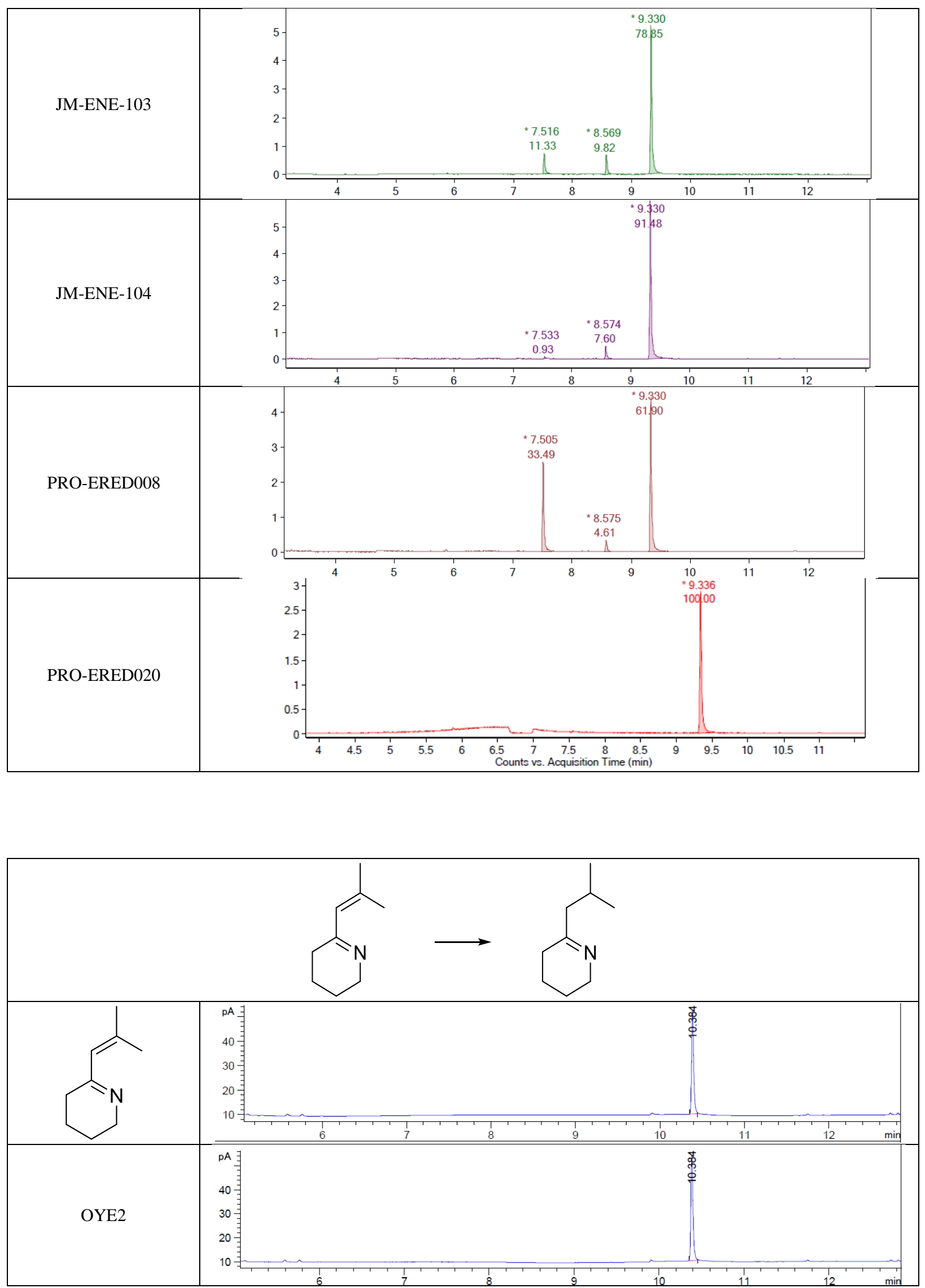


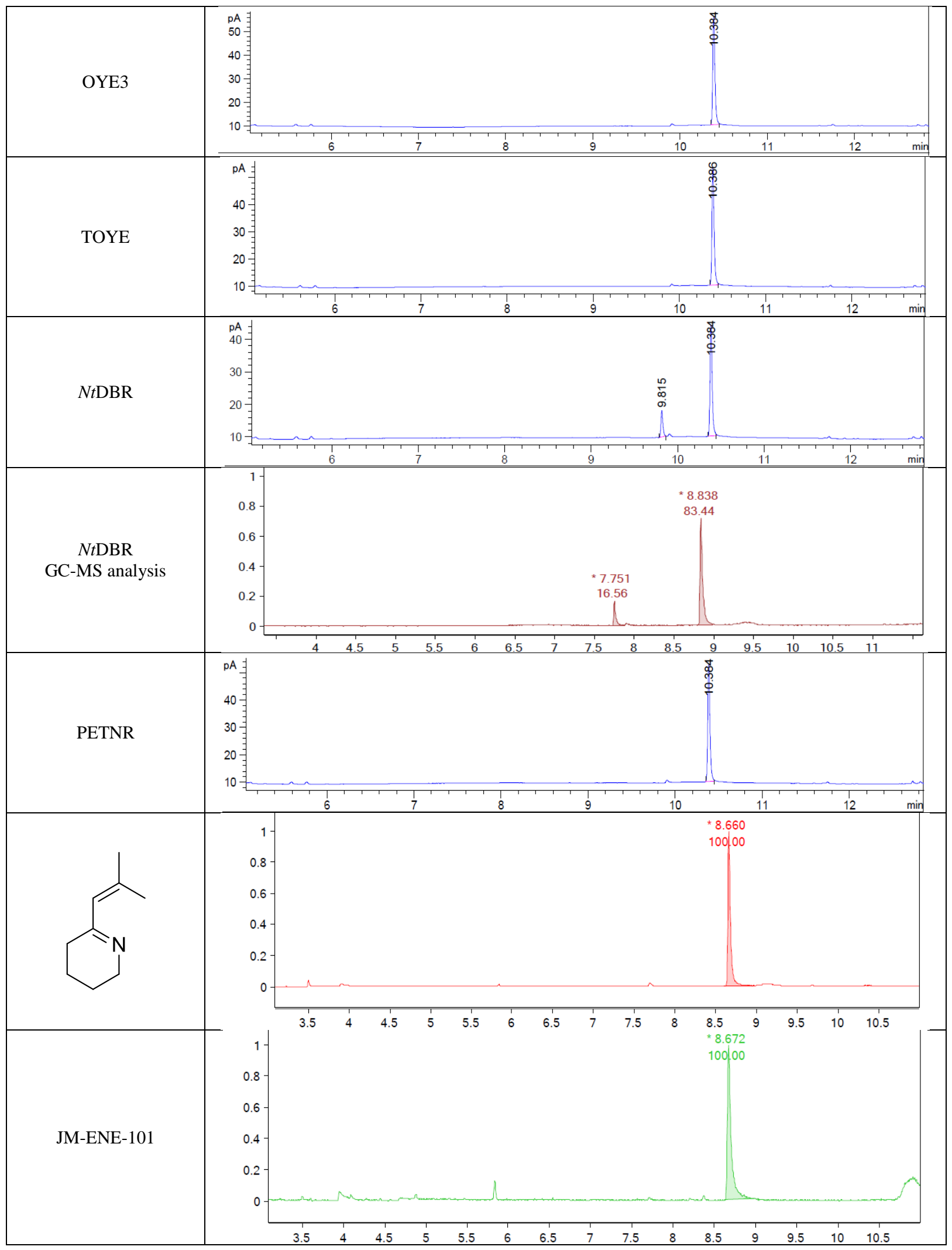




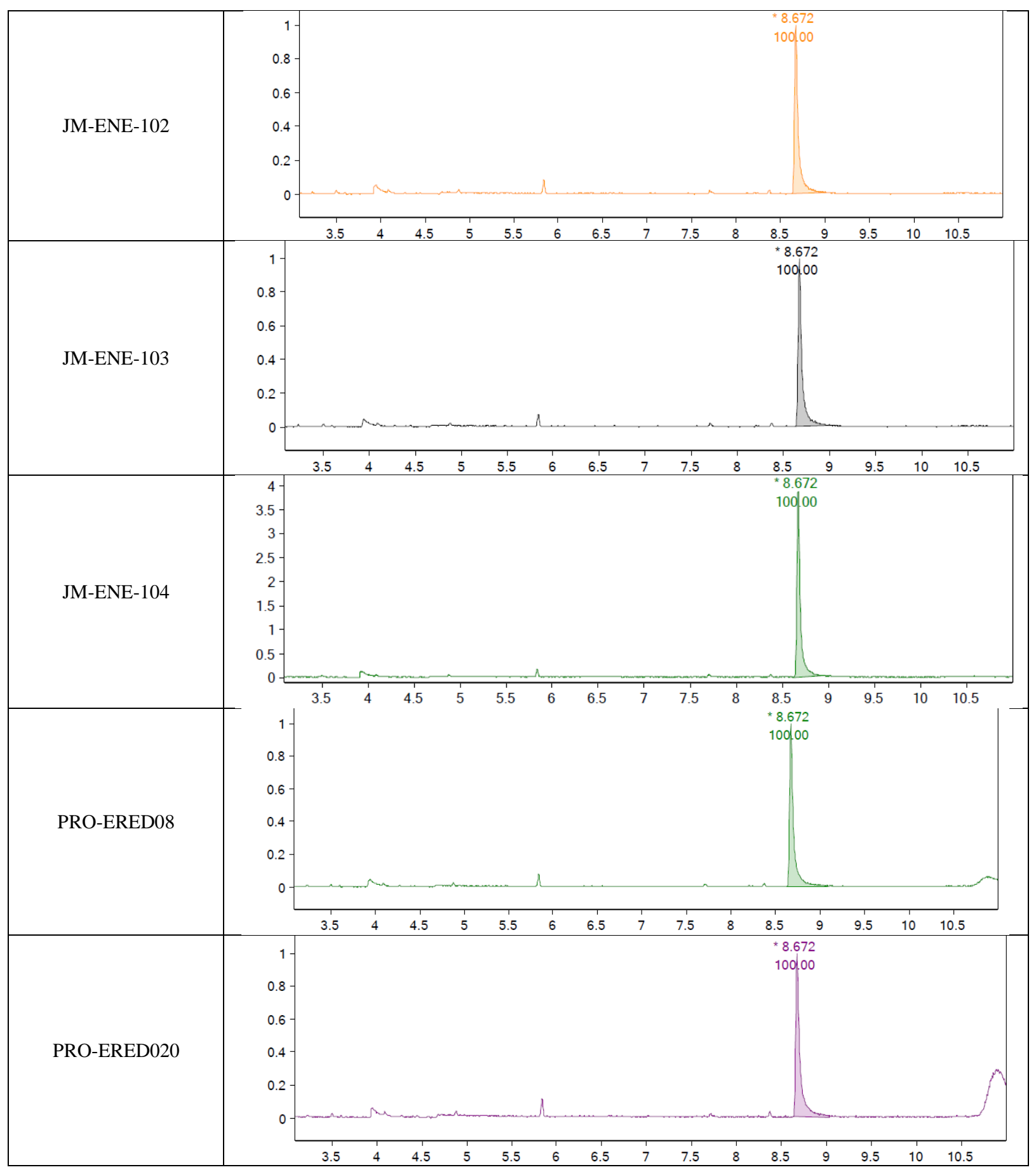




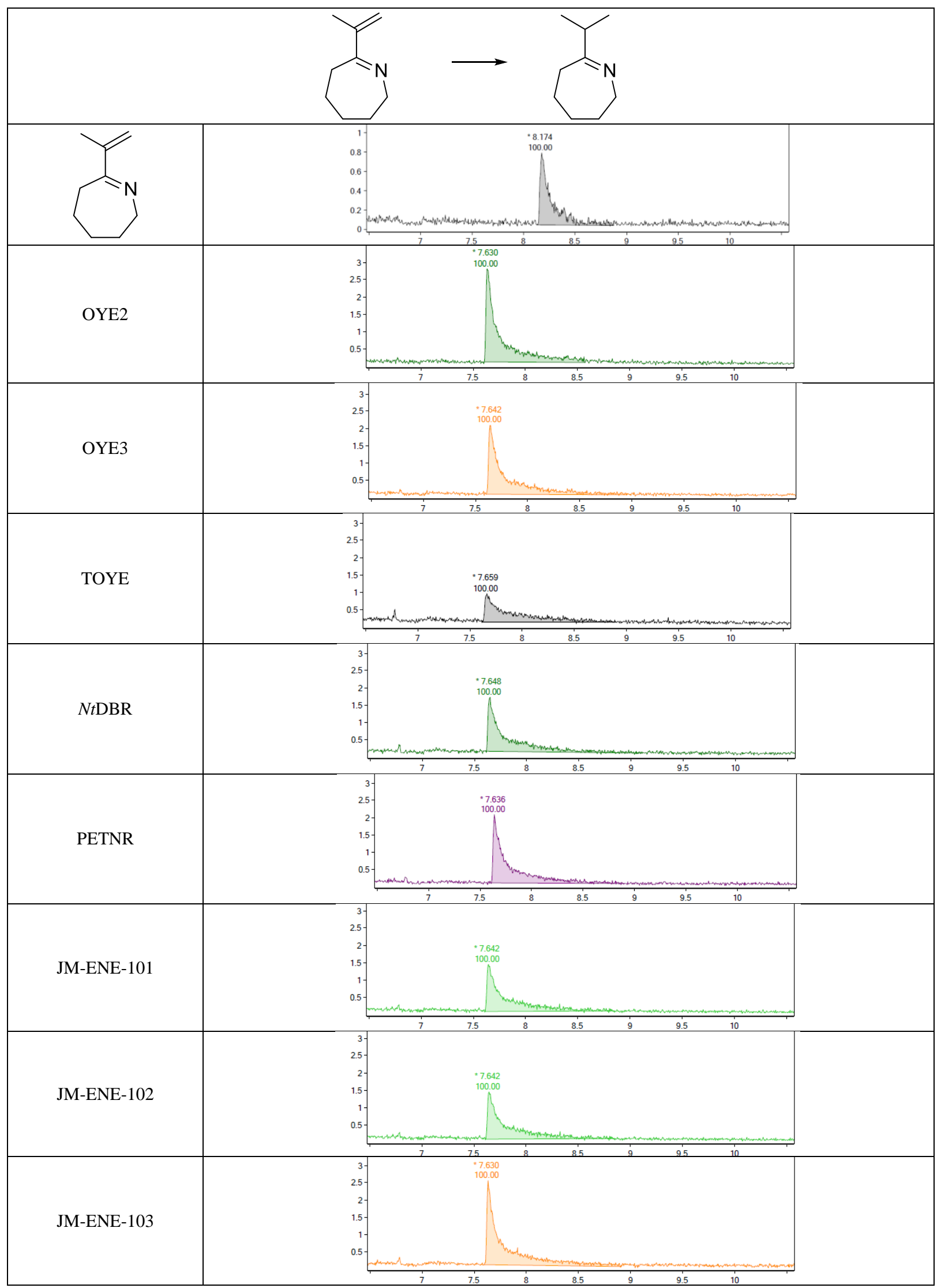



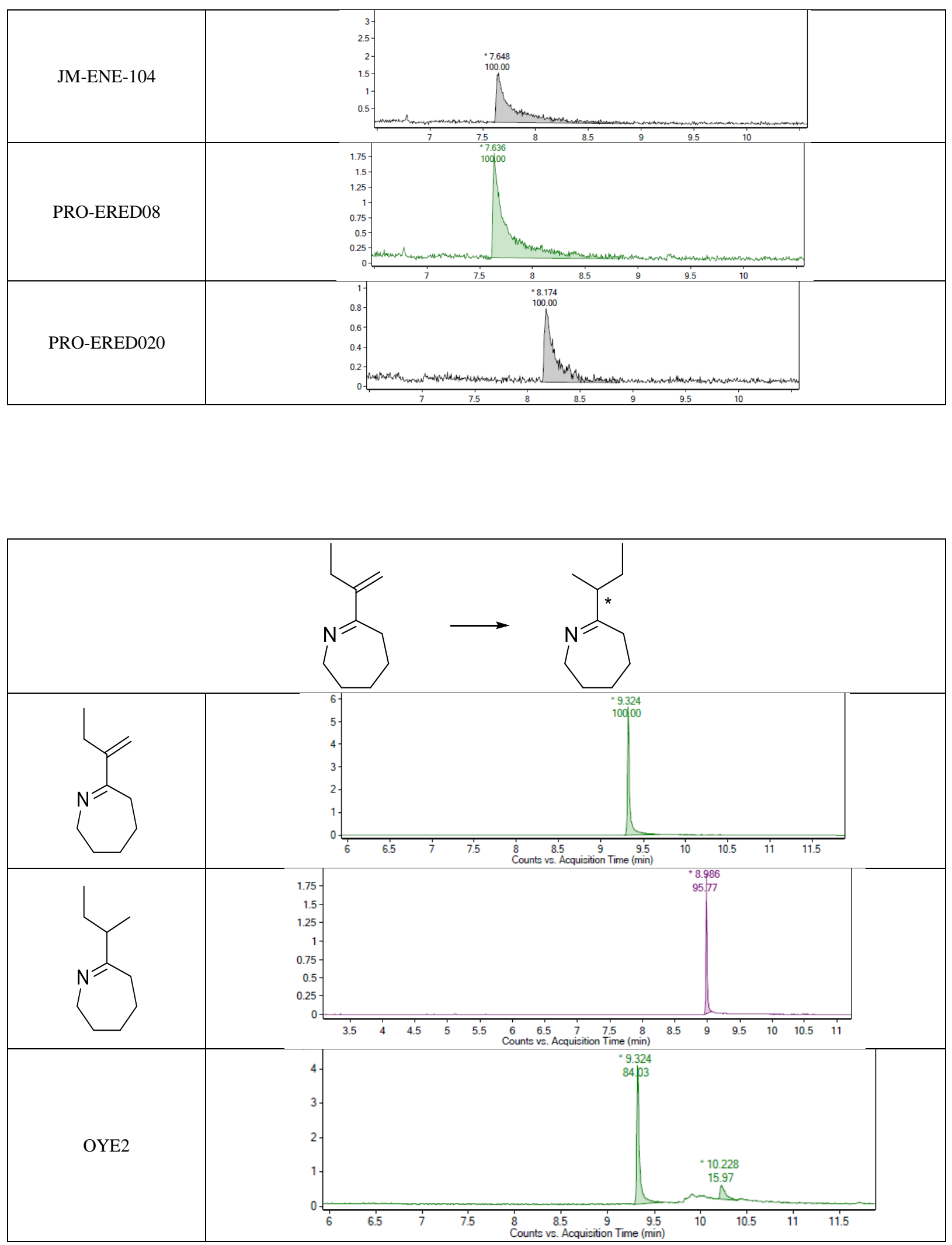


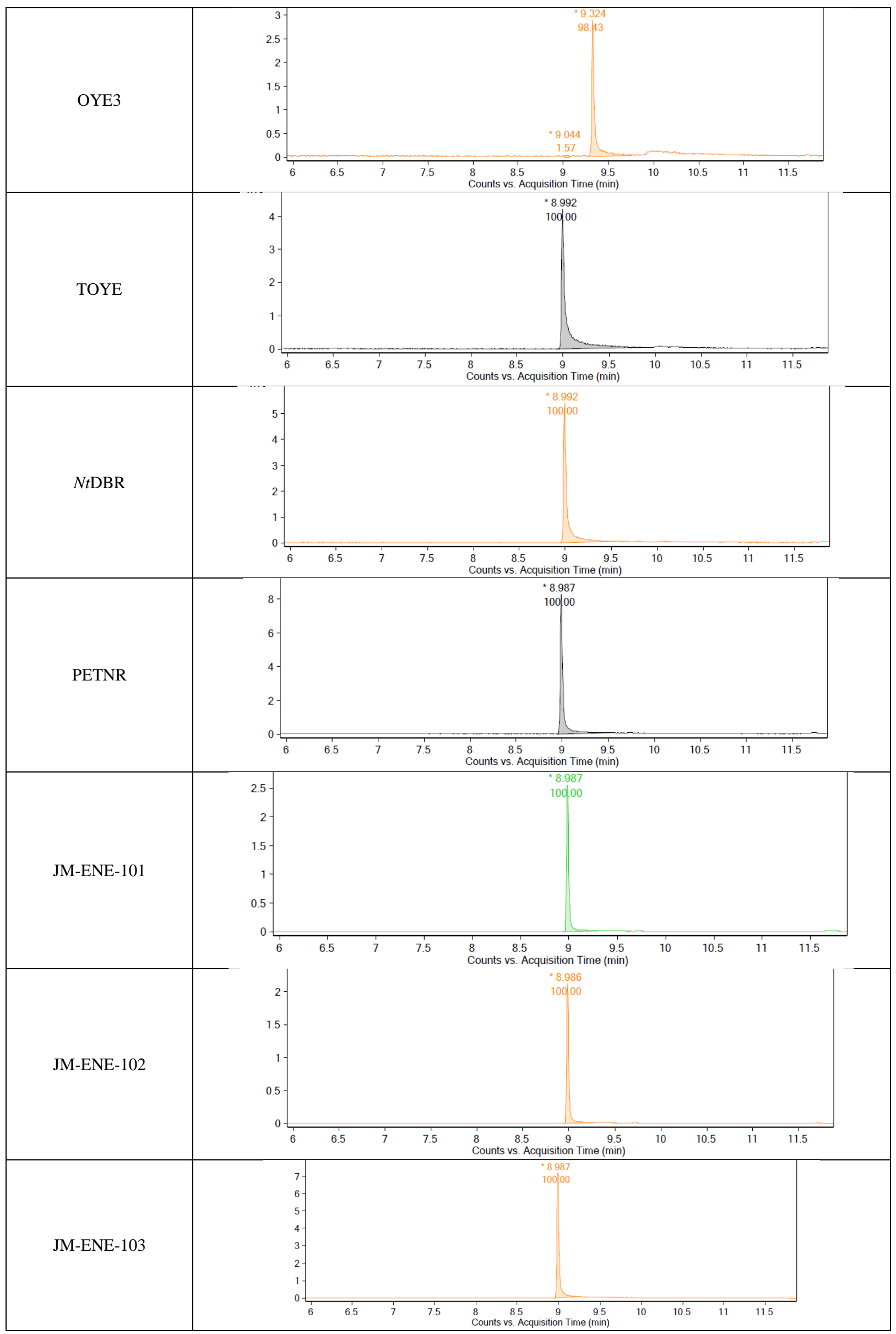



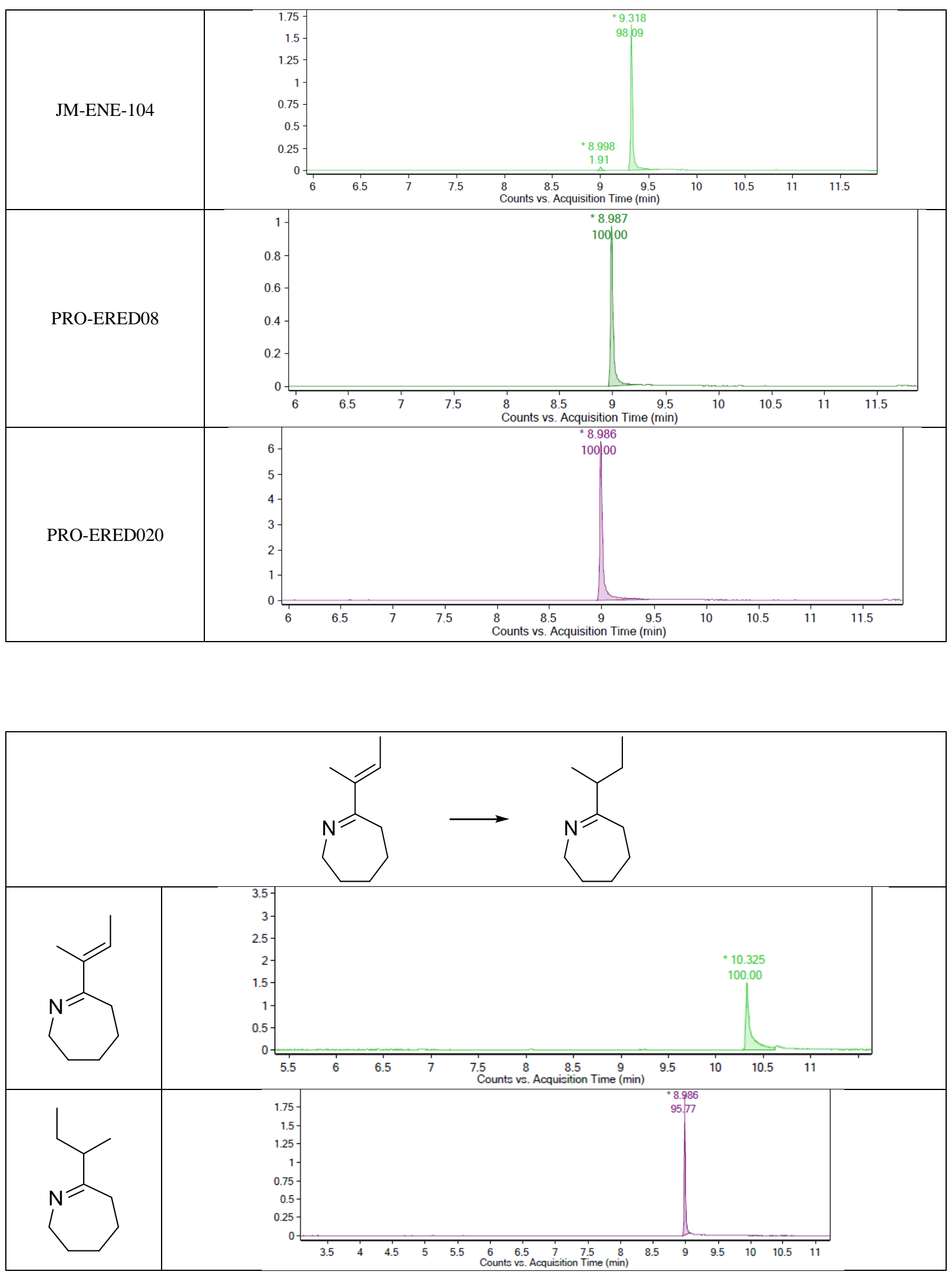


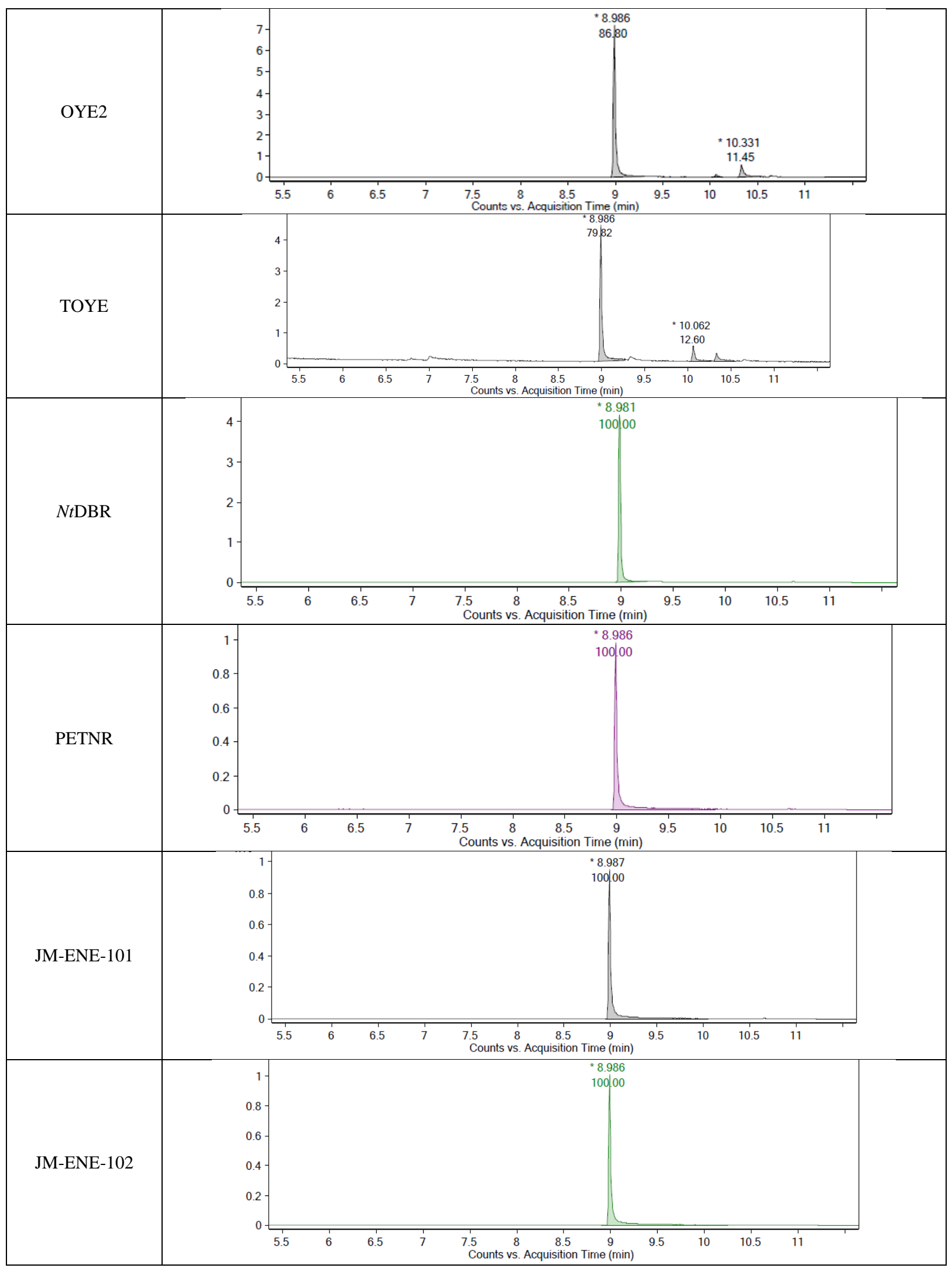



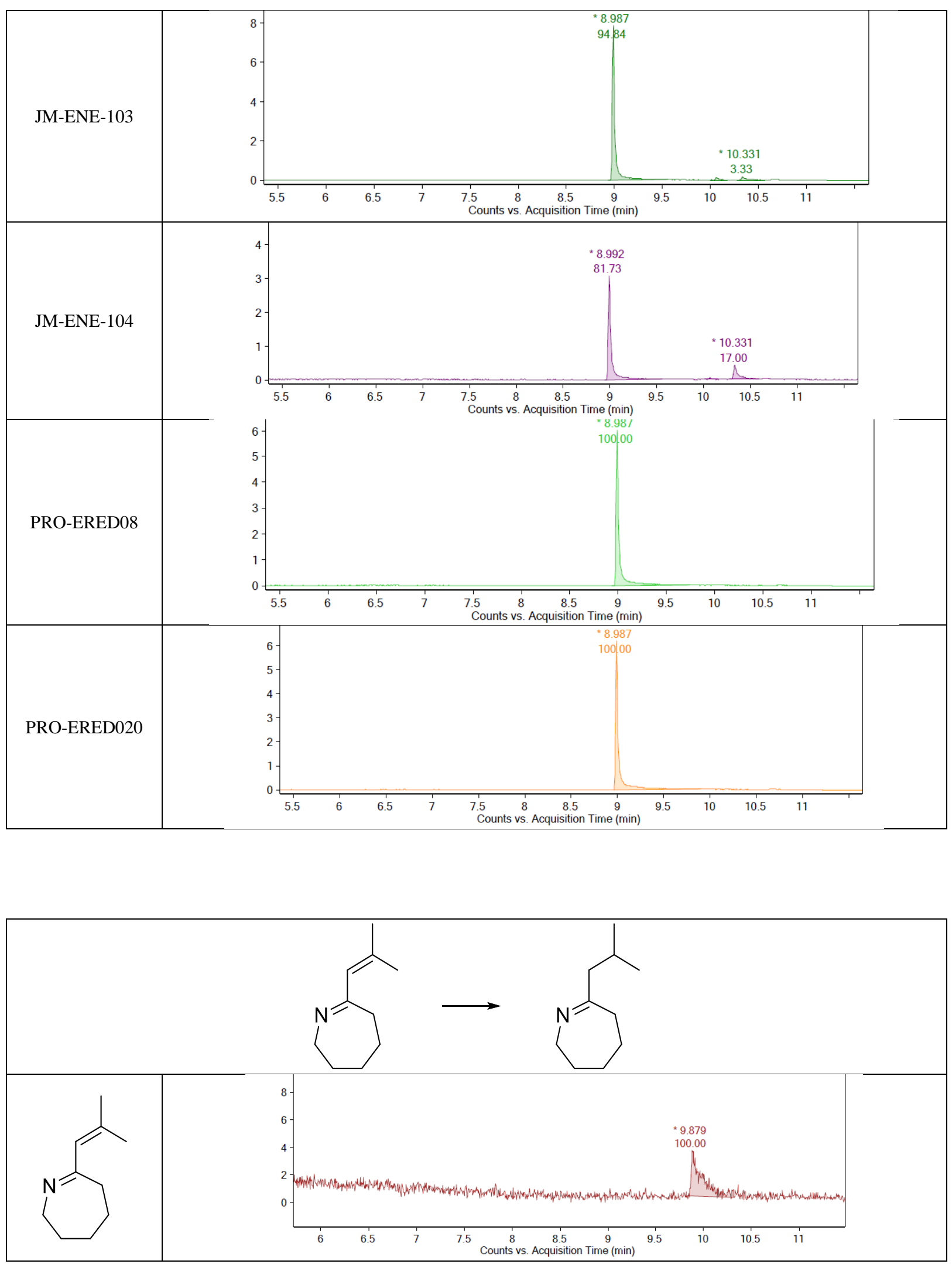


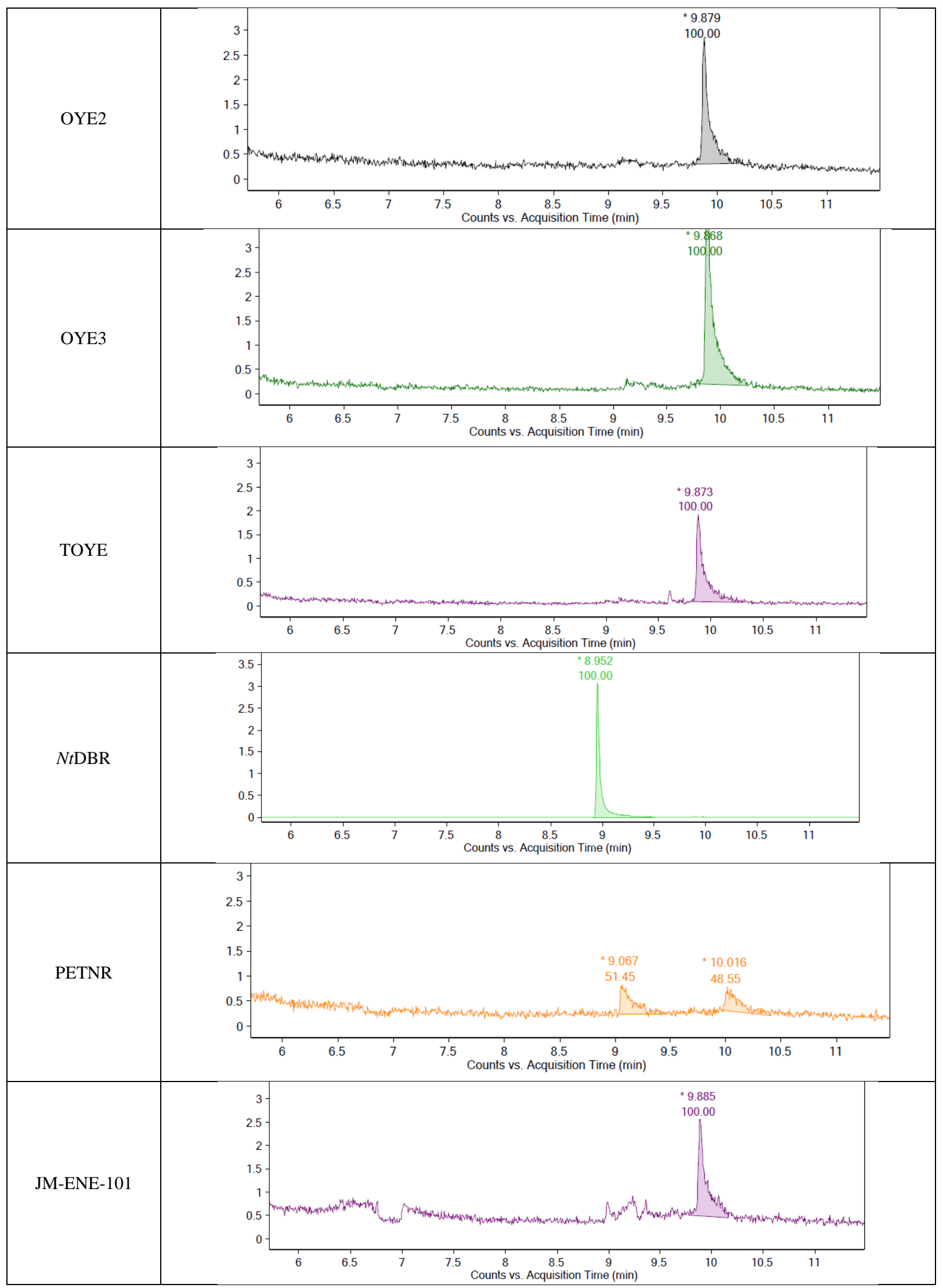




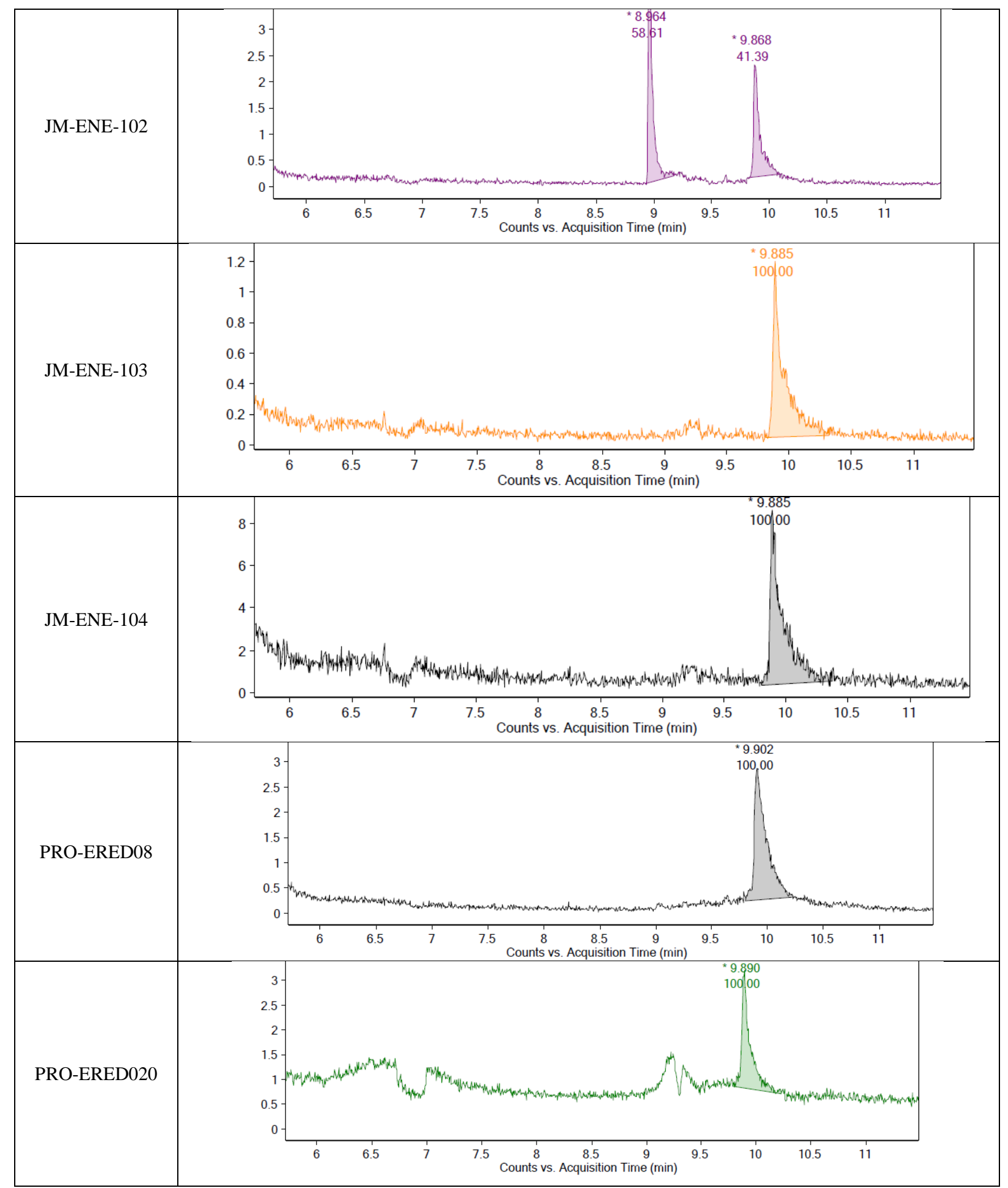




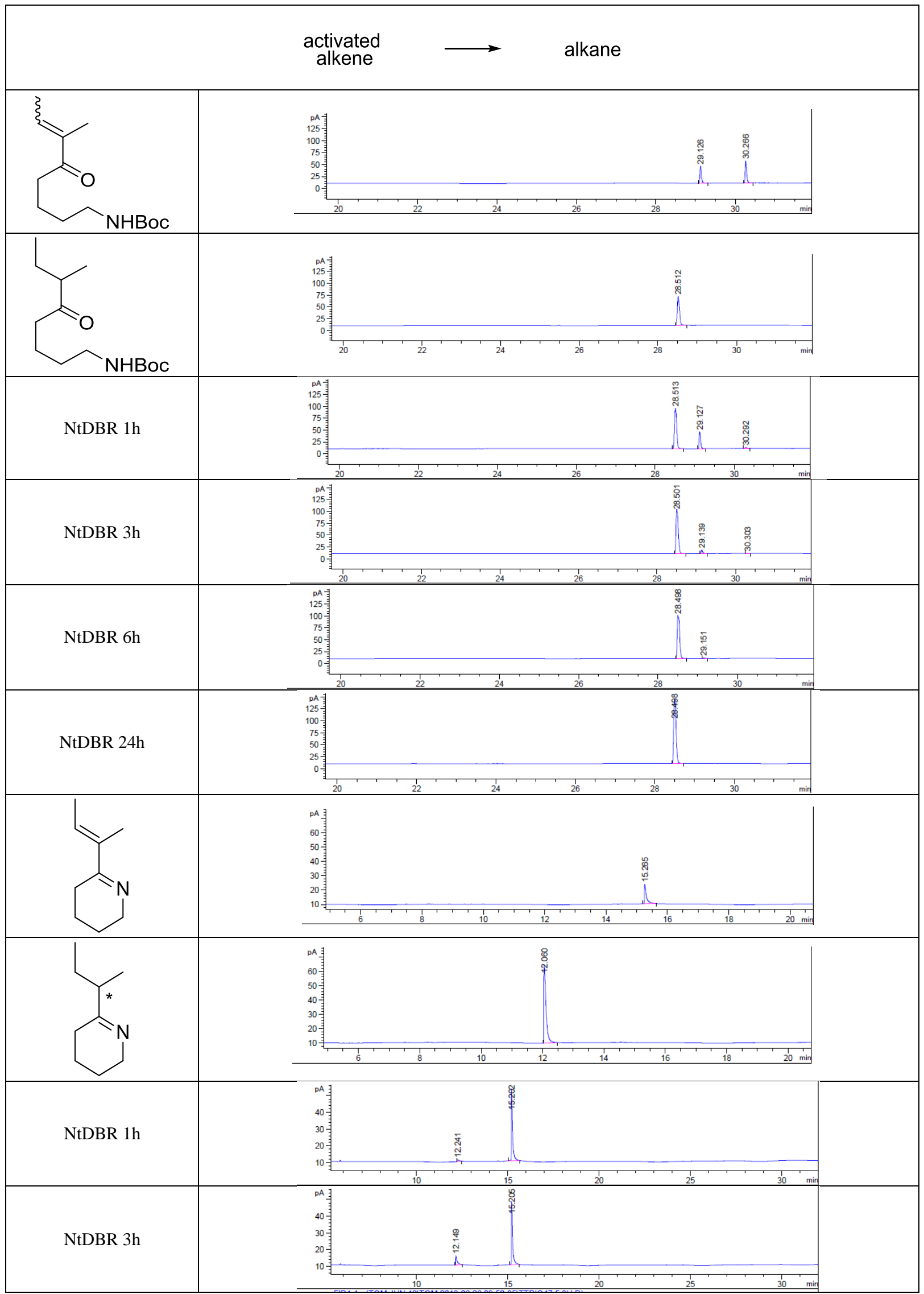




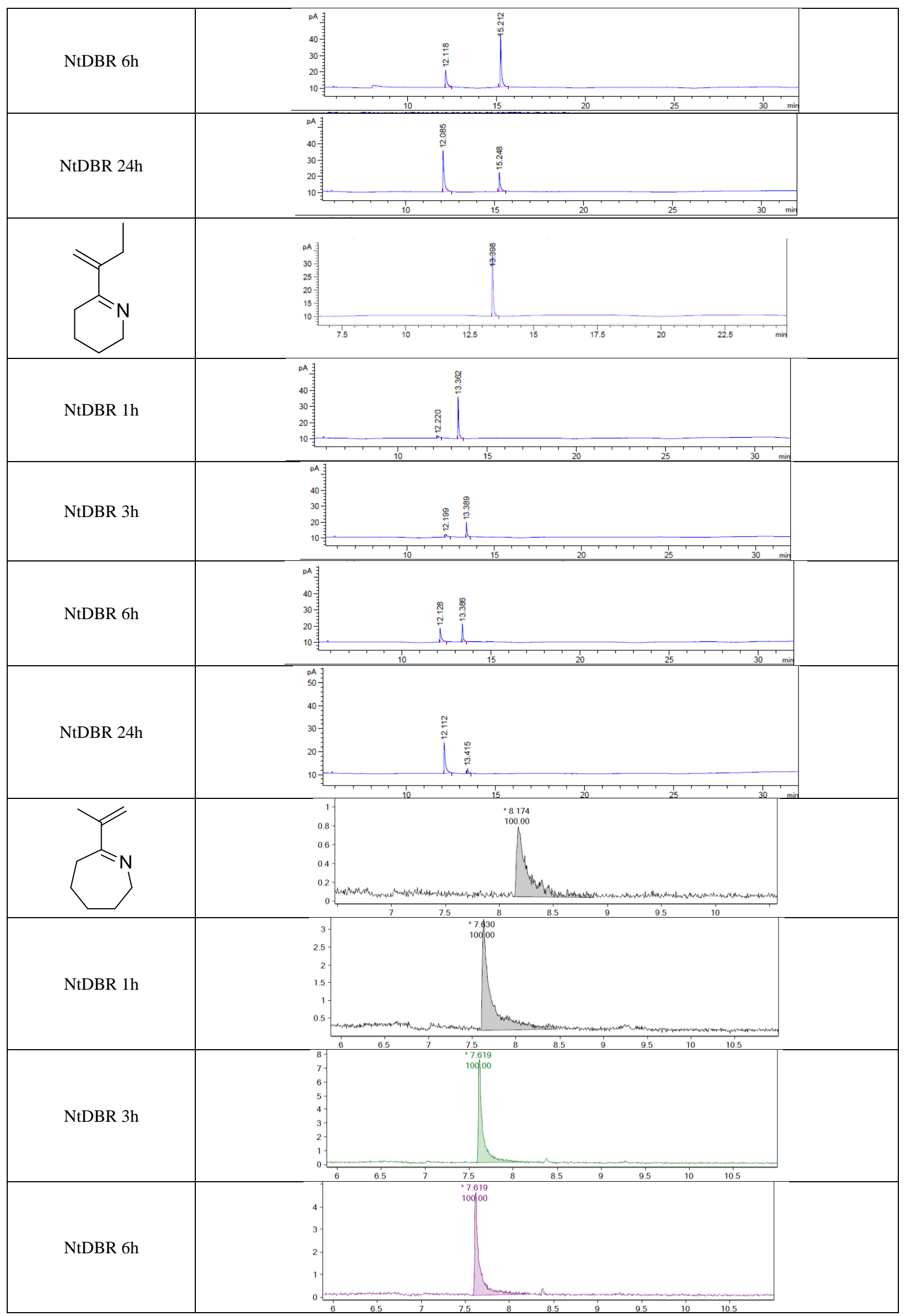




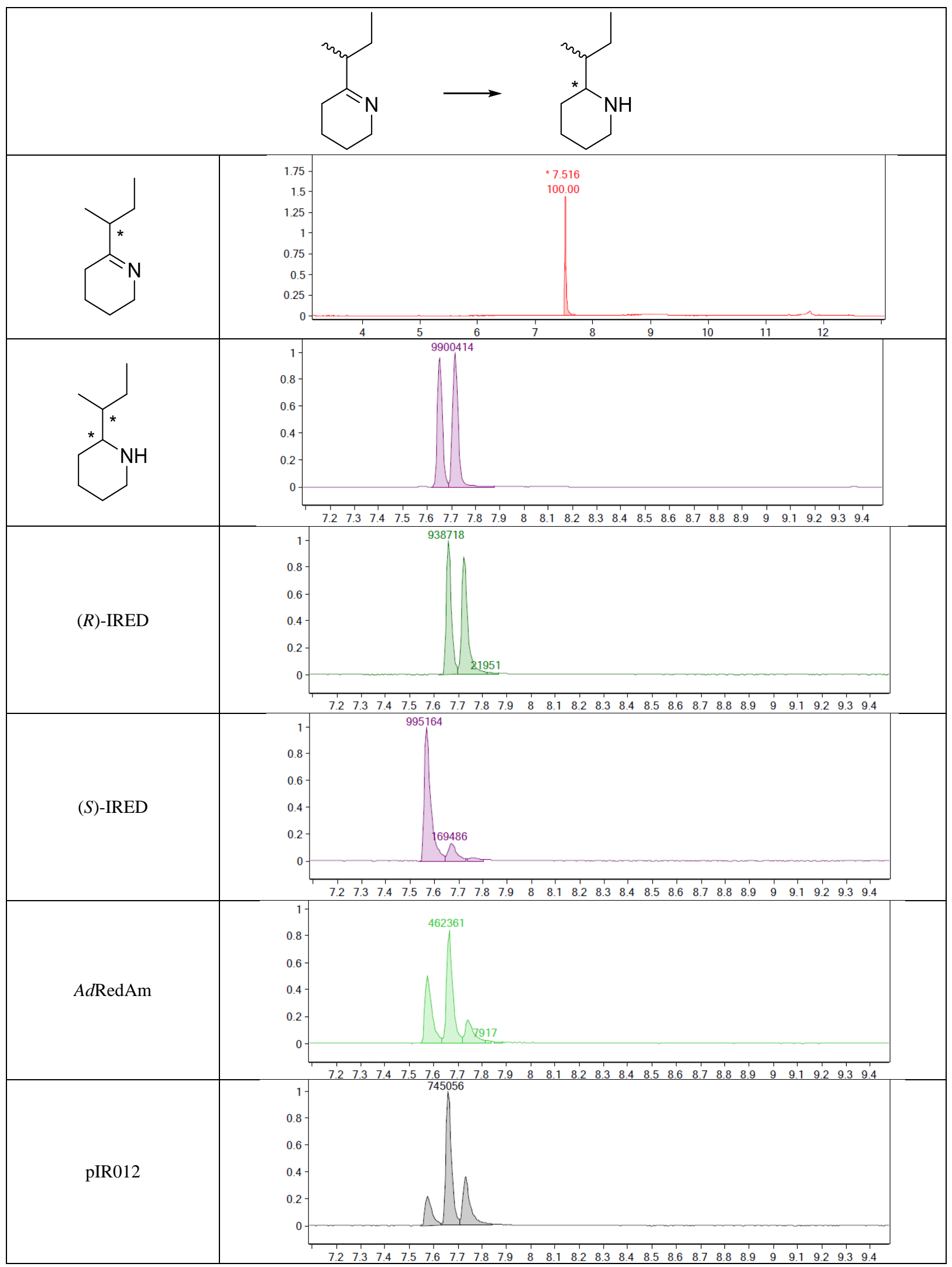




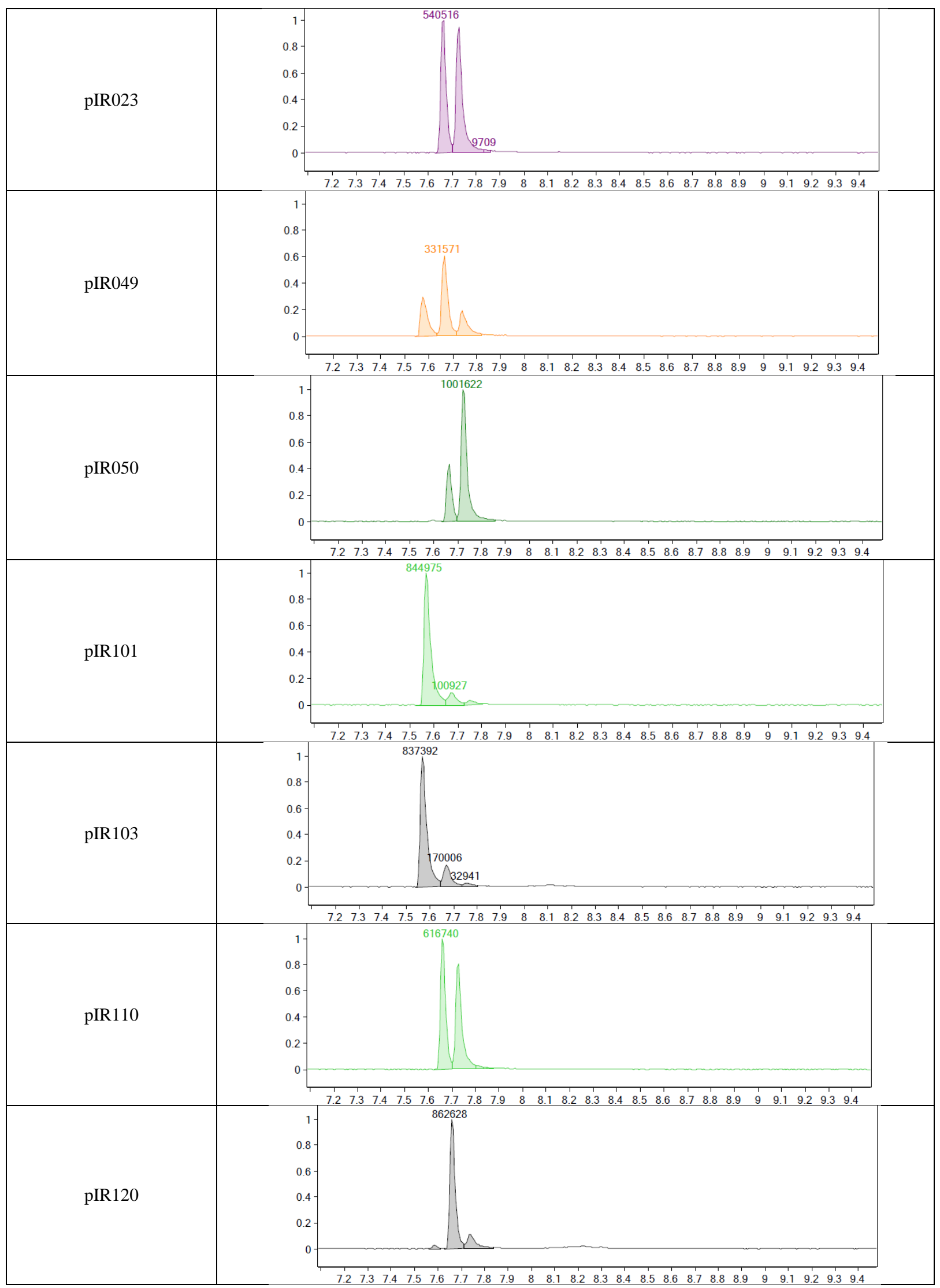




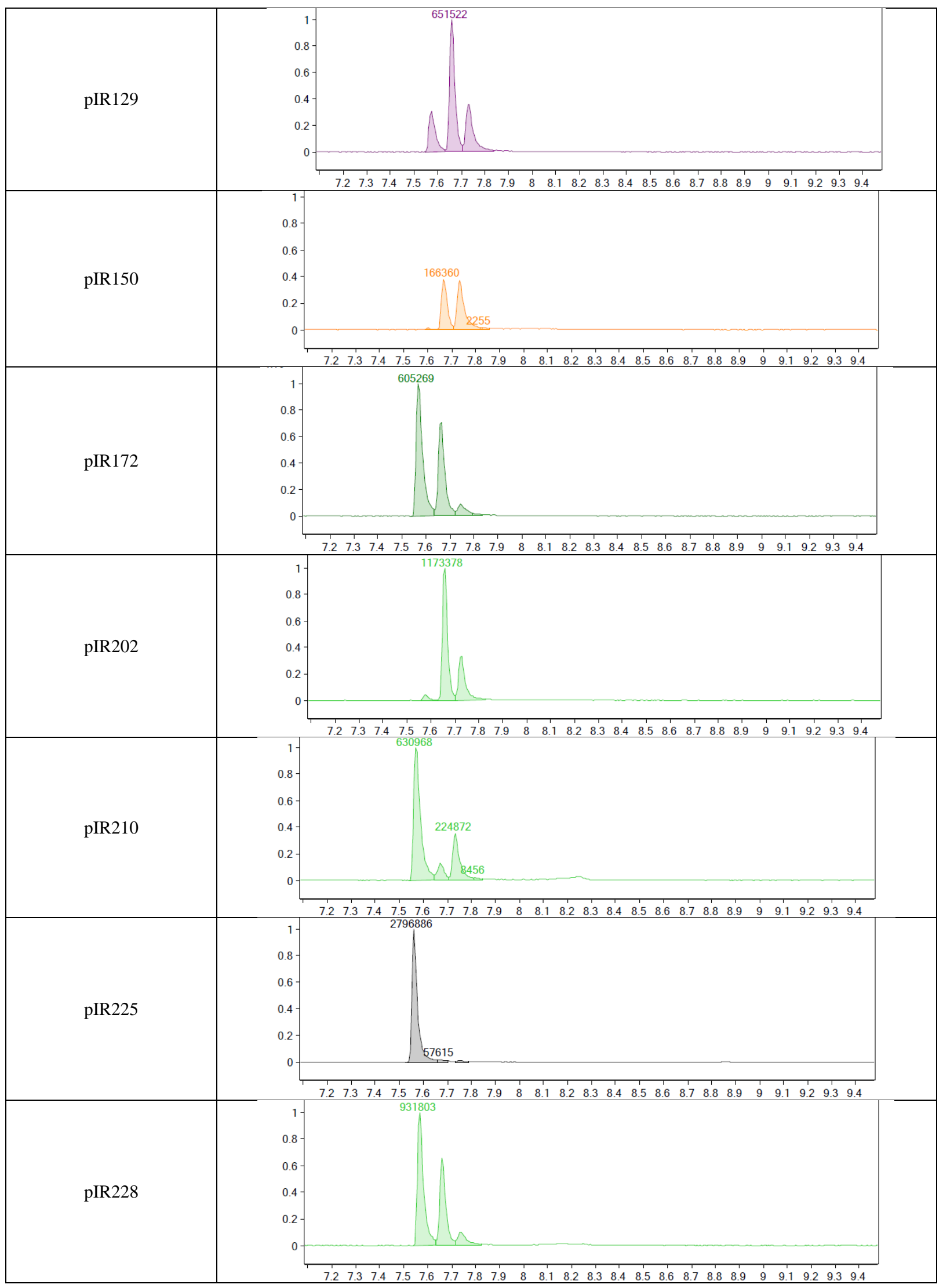




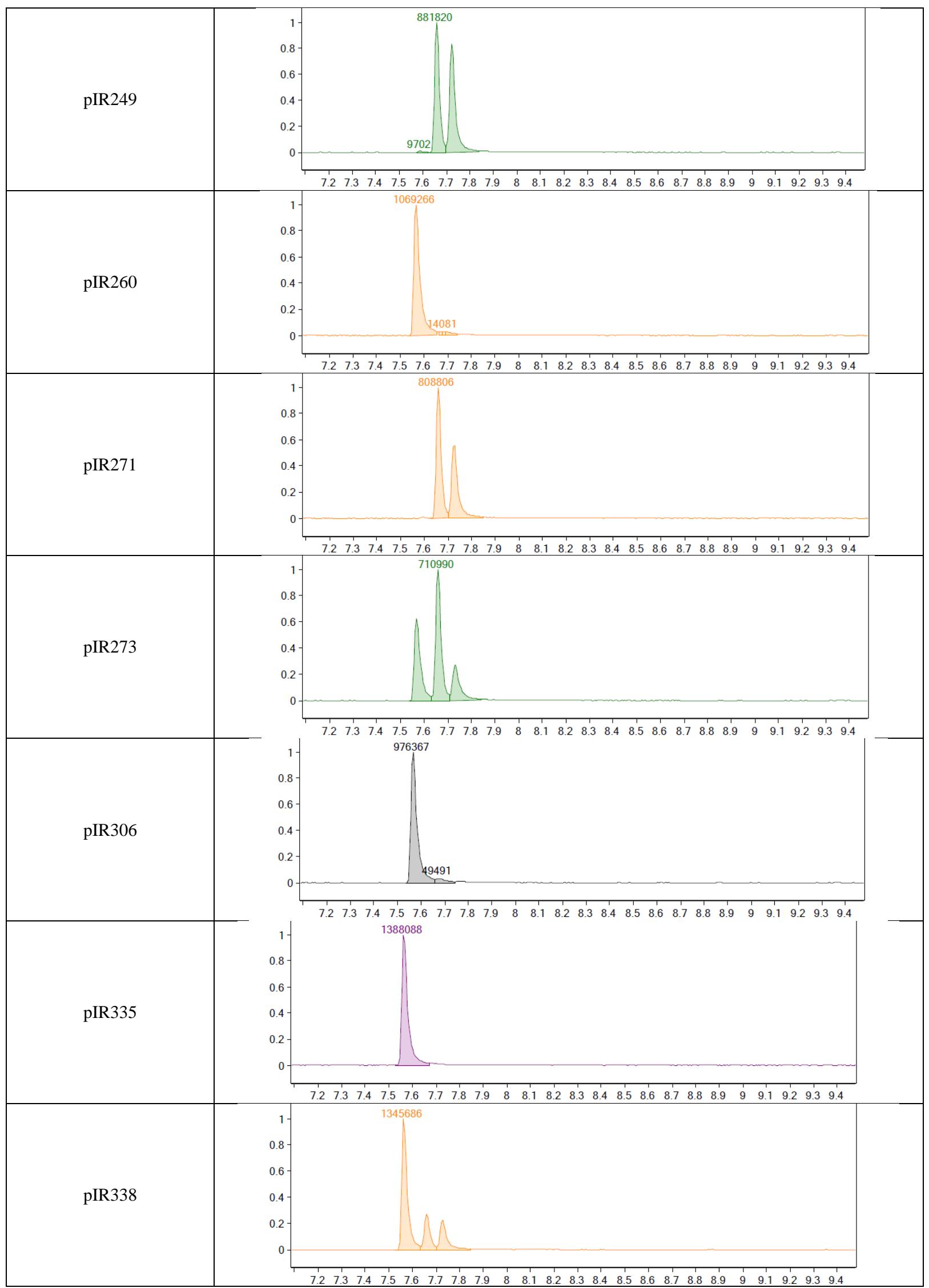




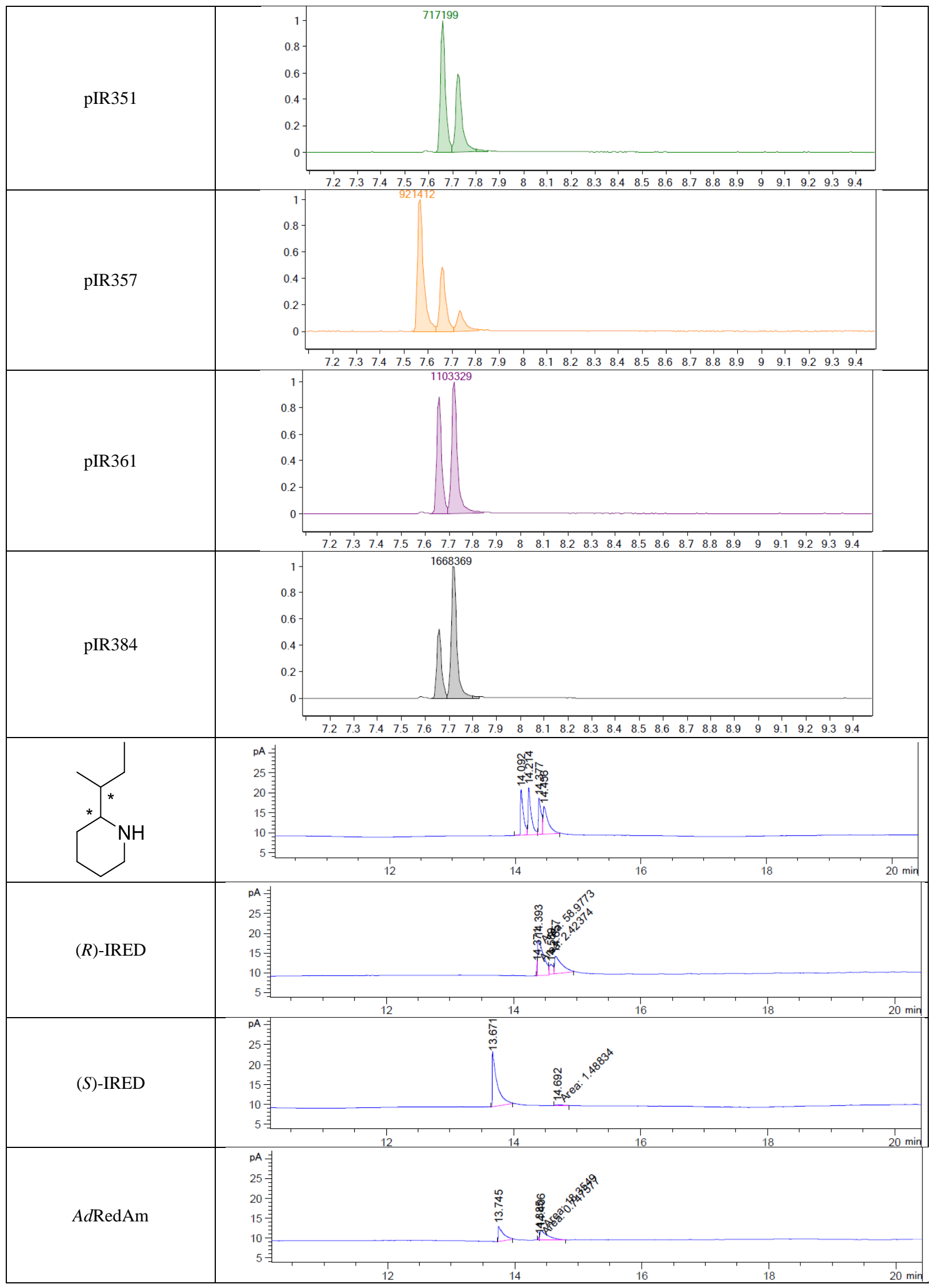




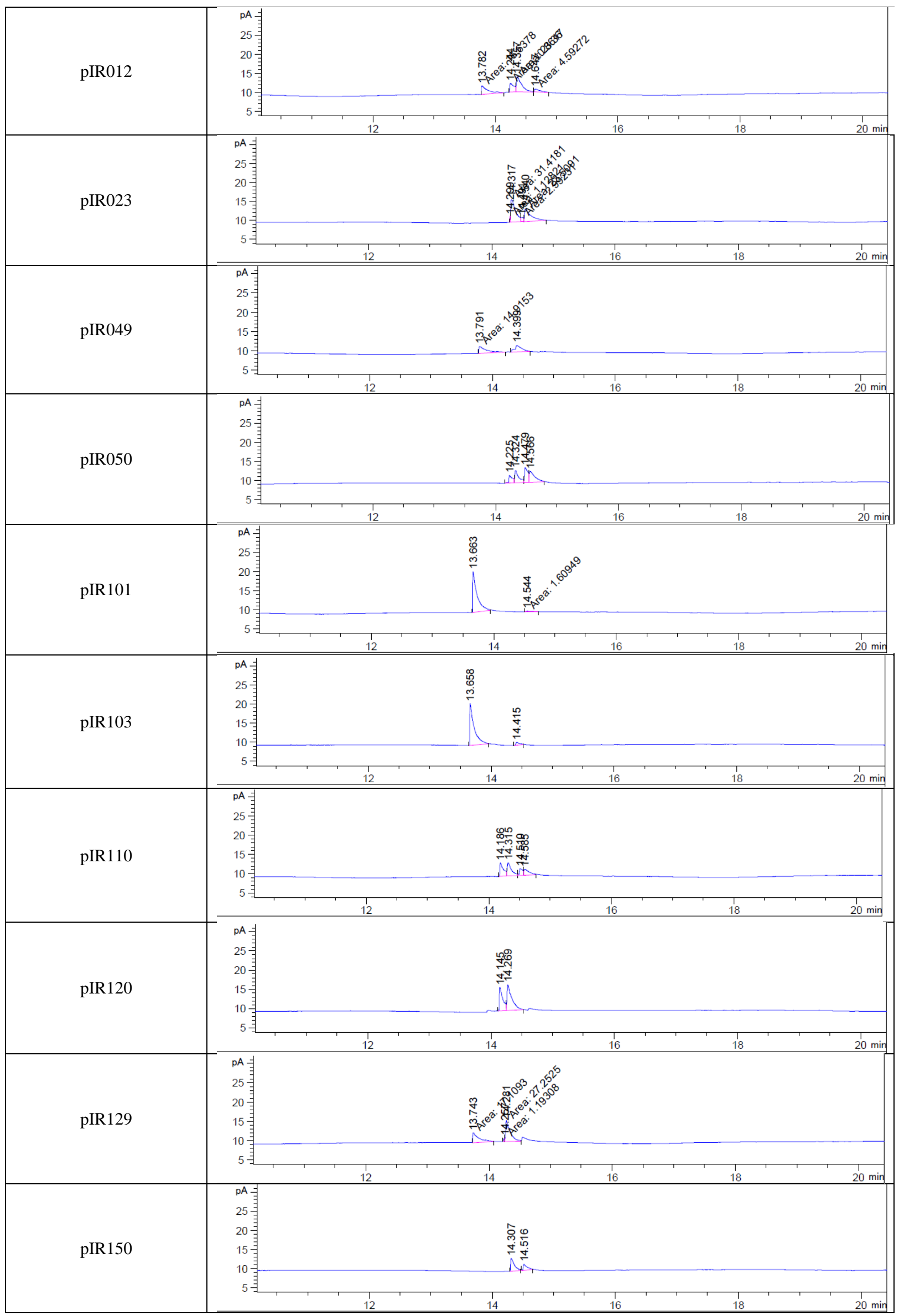




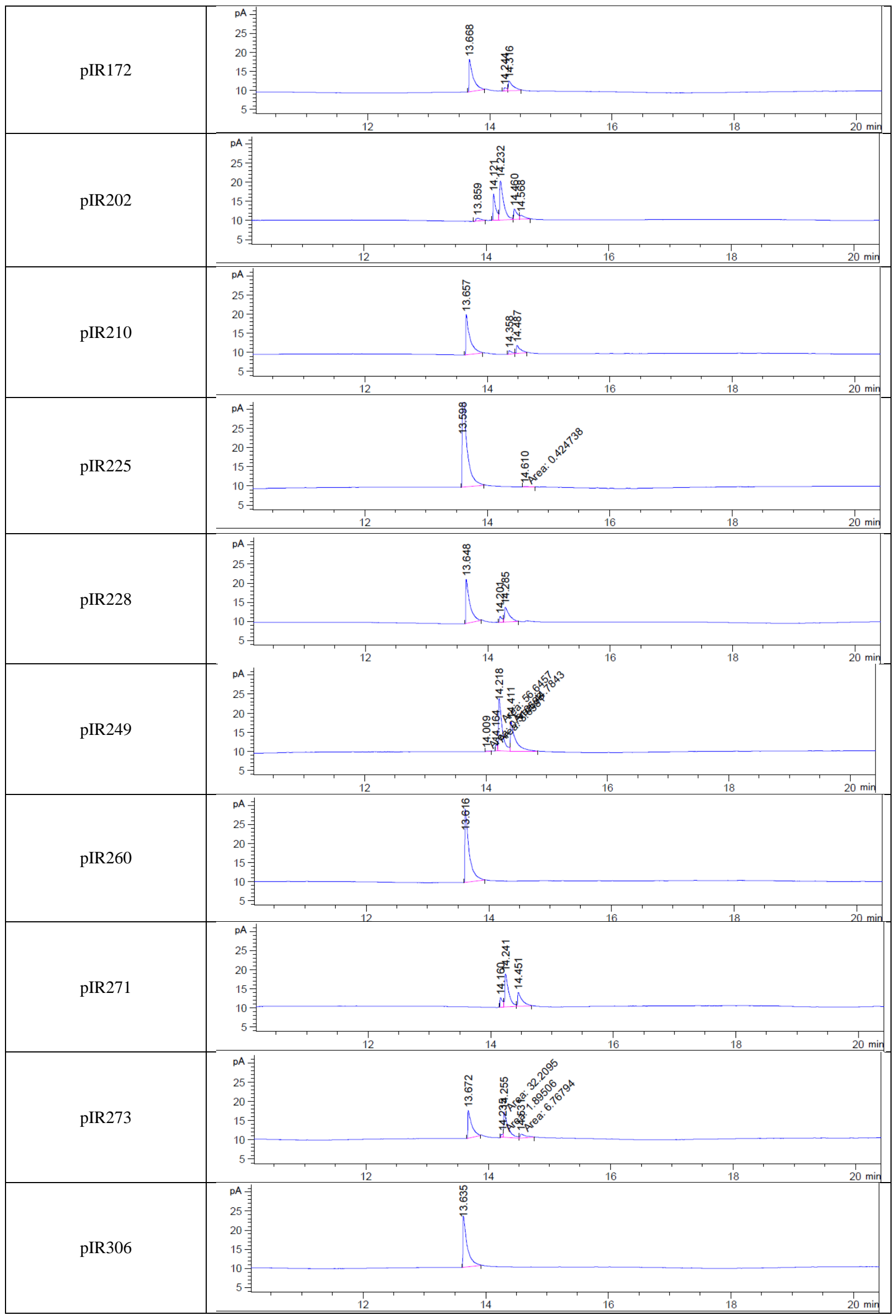




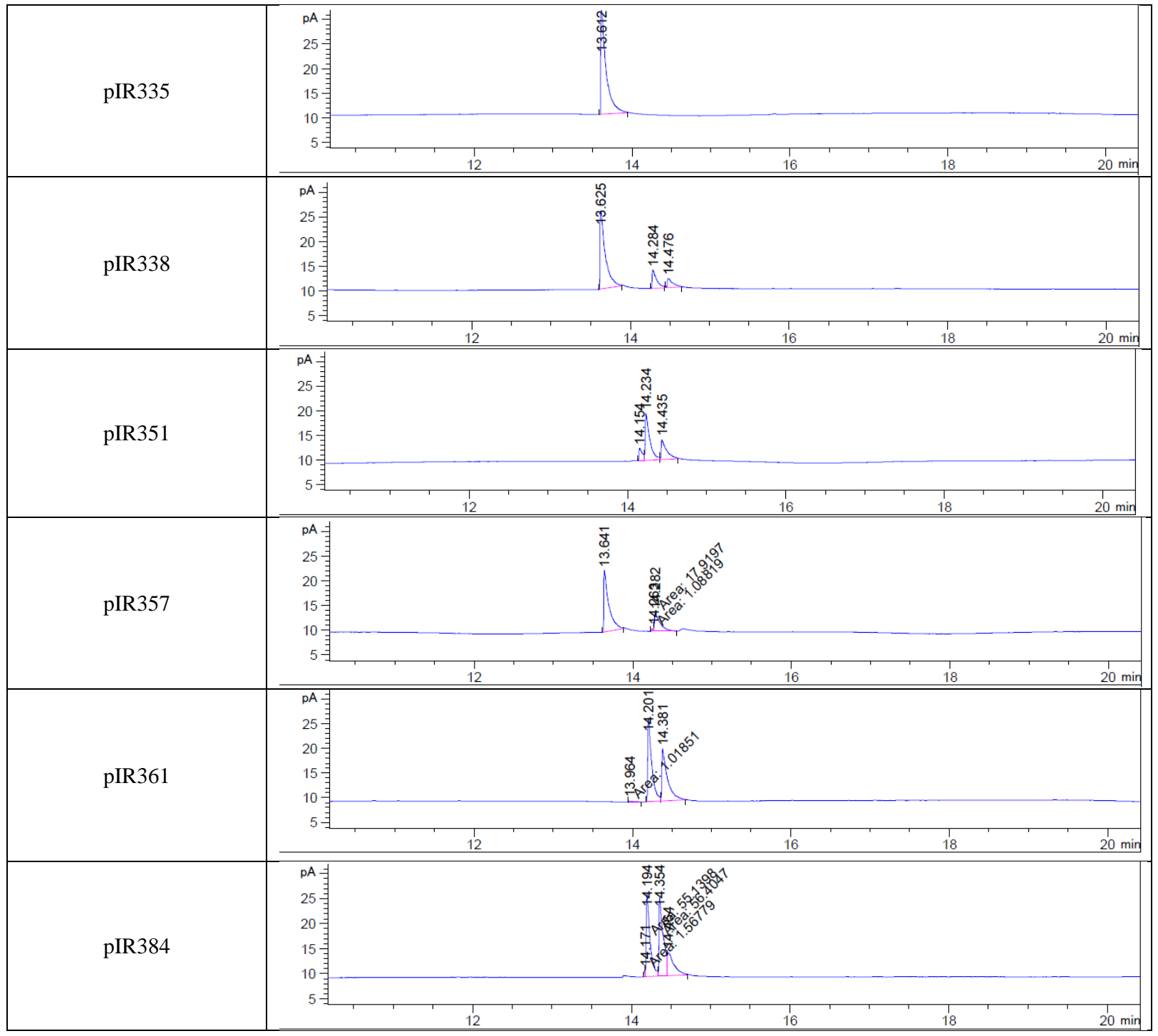

4.2.4 ERED-IRED telescopic synthesis of saturated $N$-heterocycles

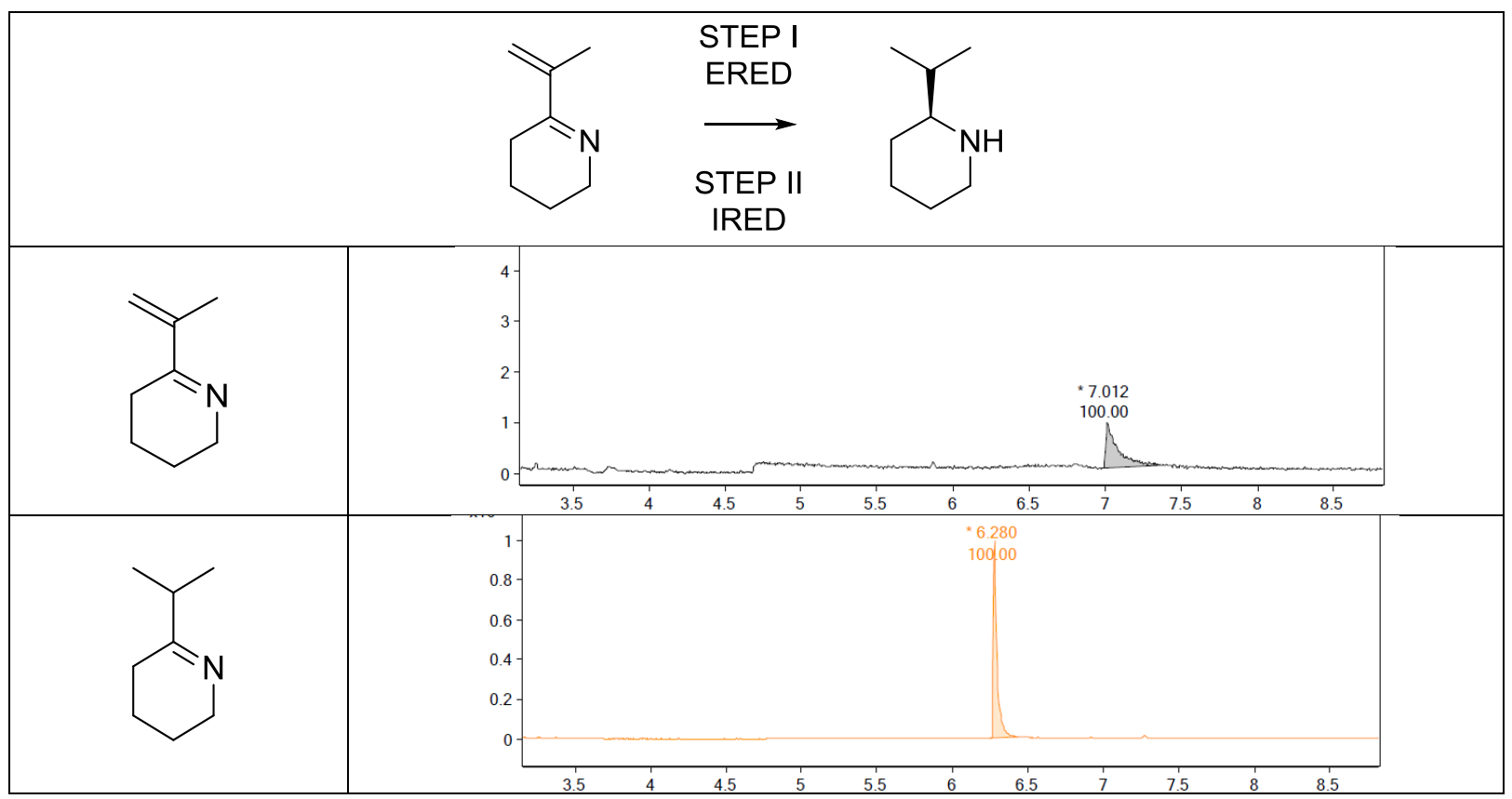



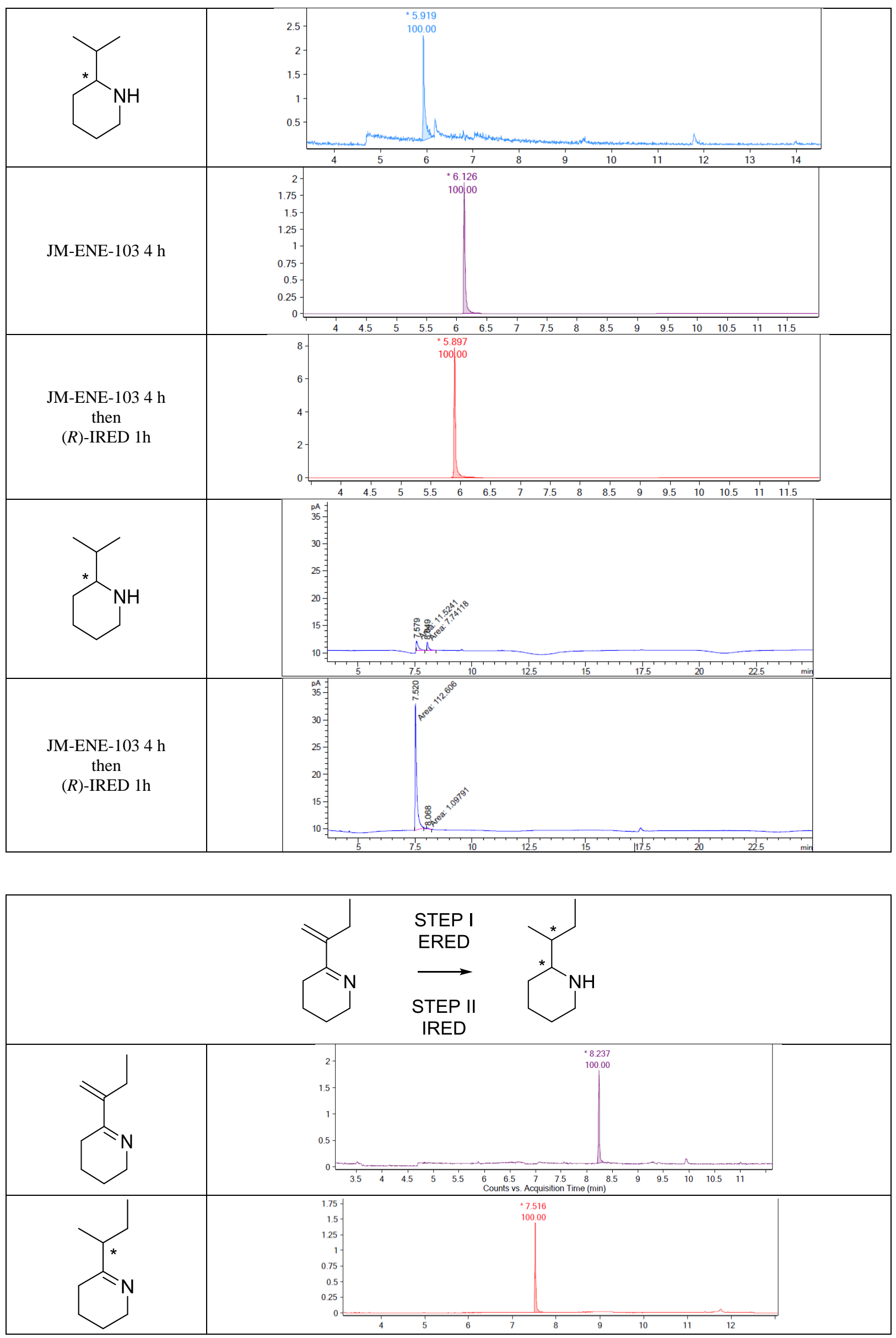


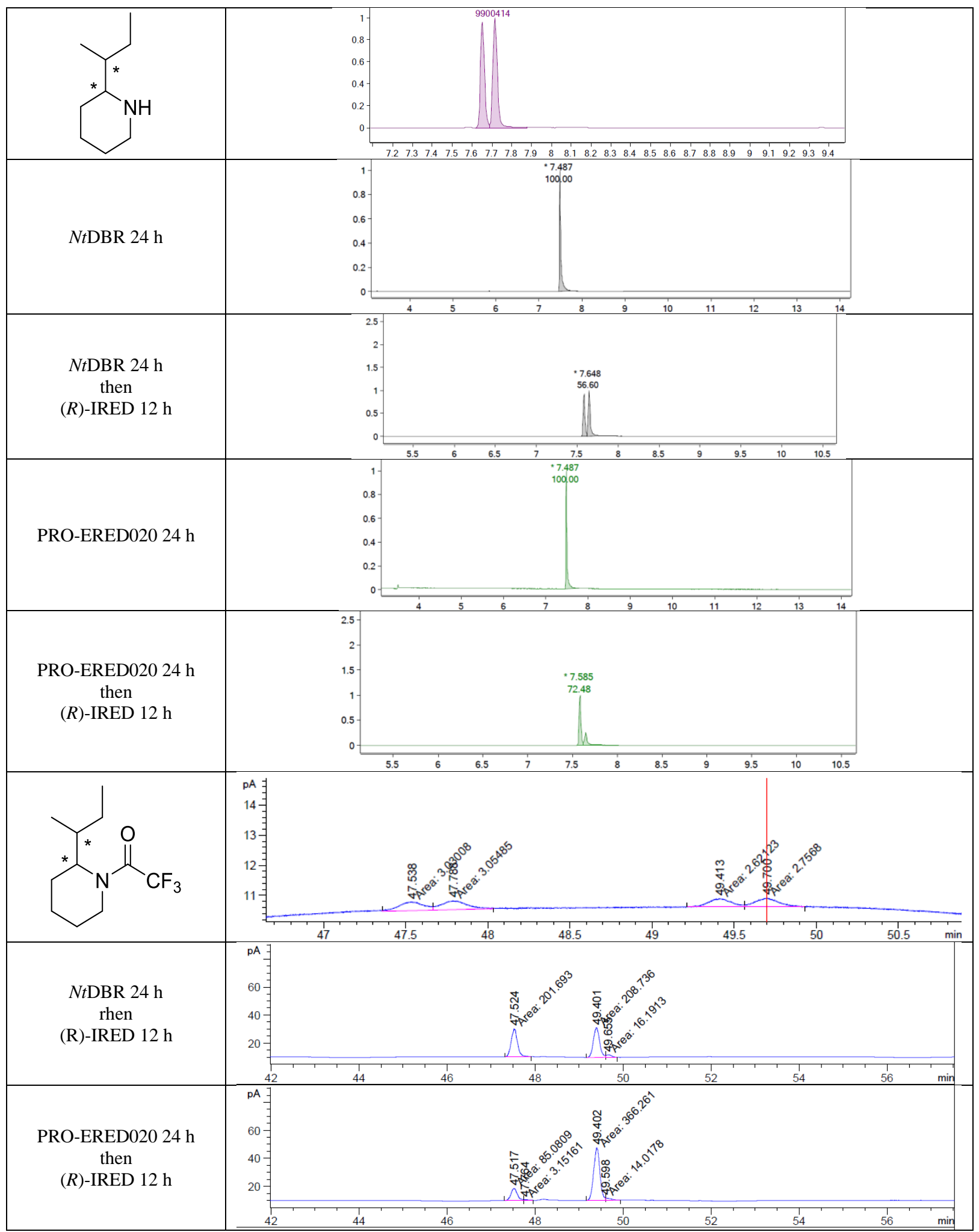




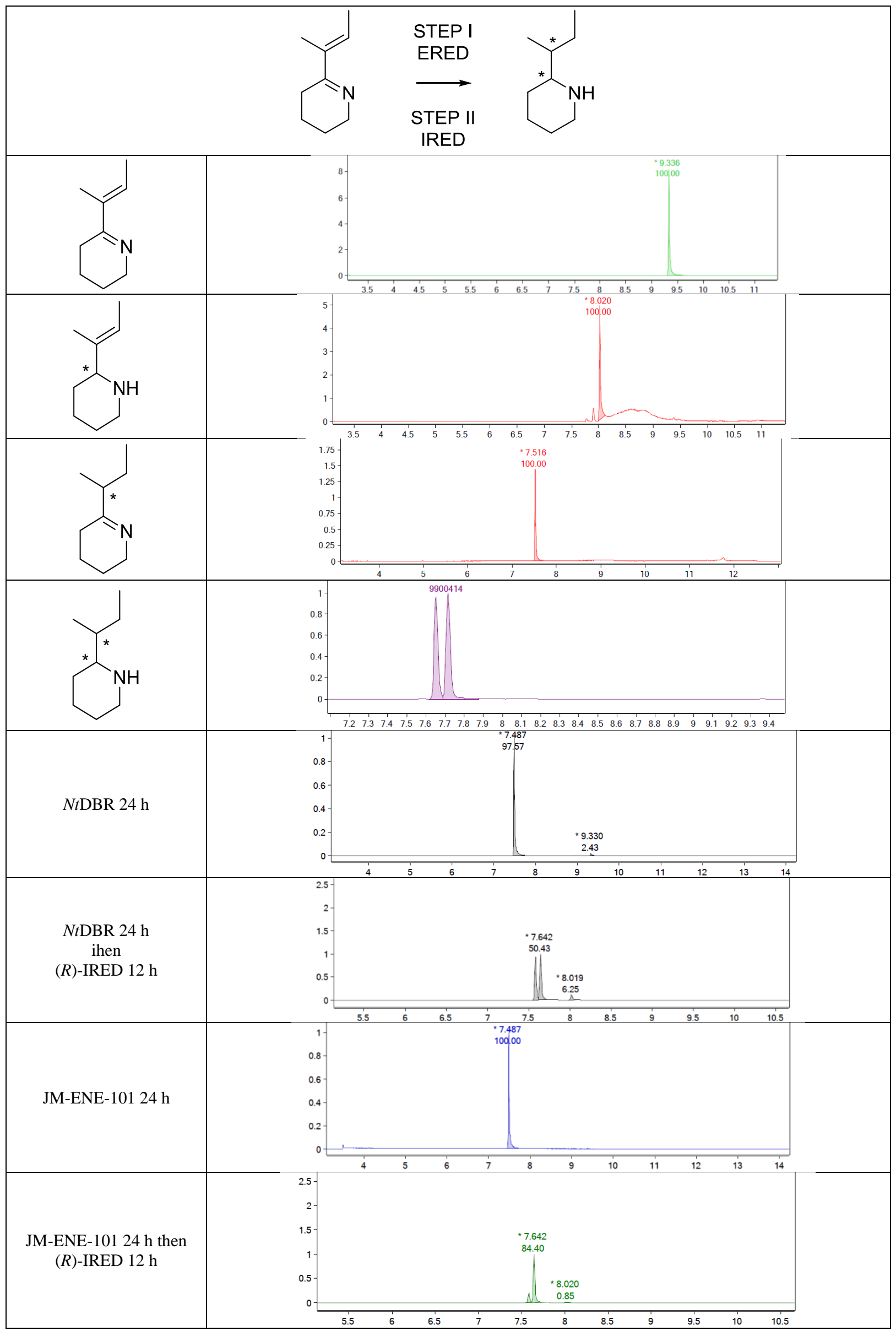




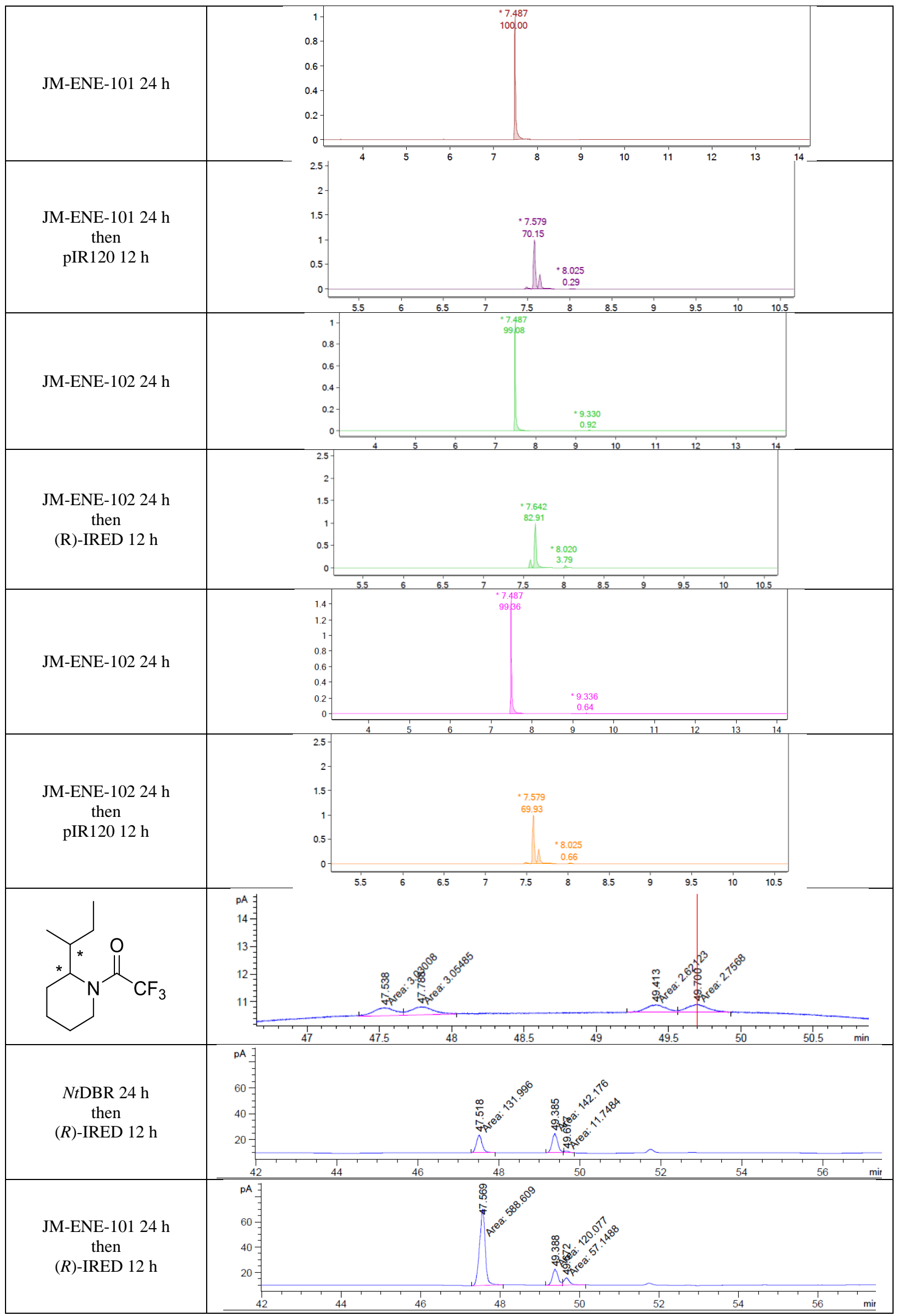



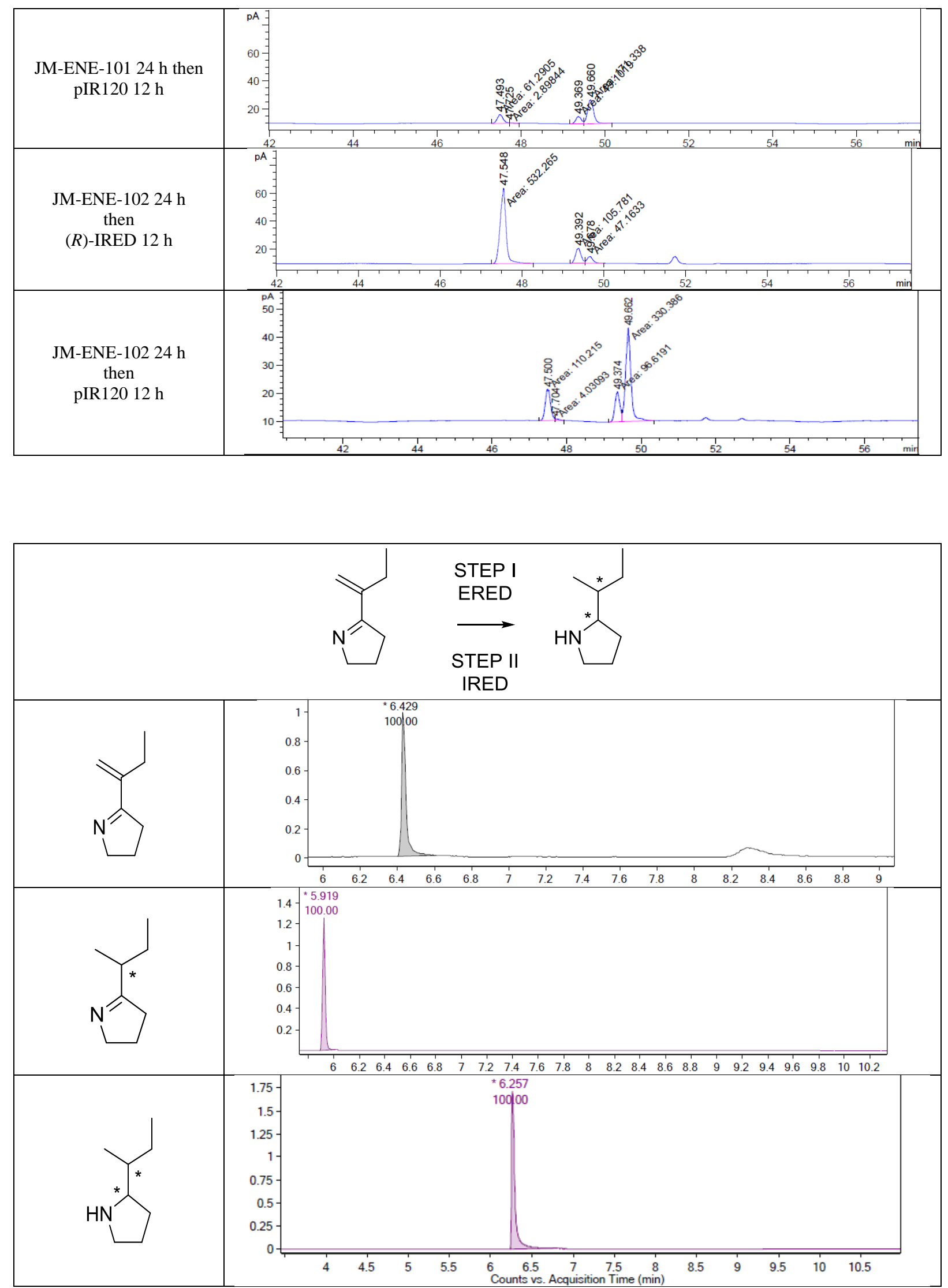

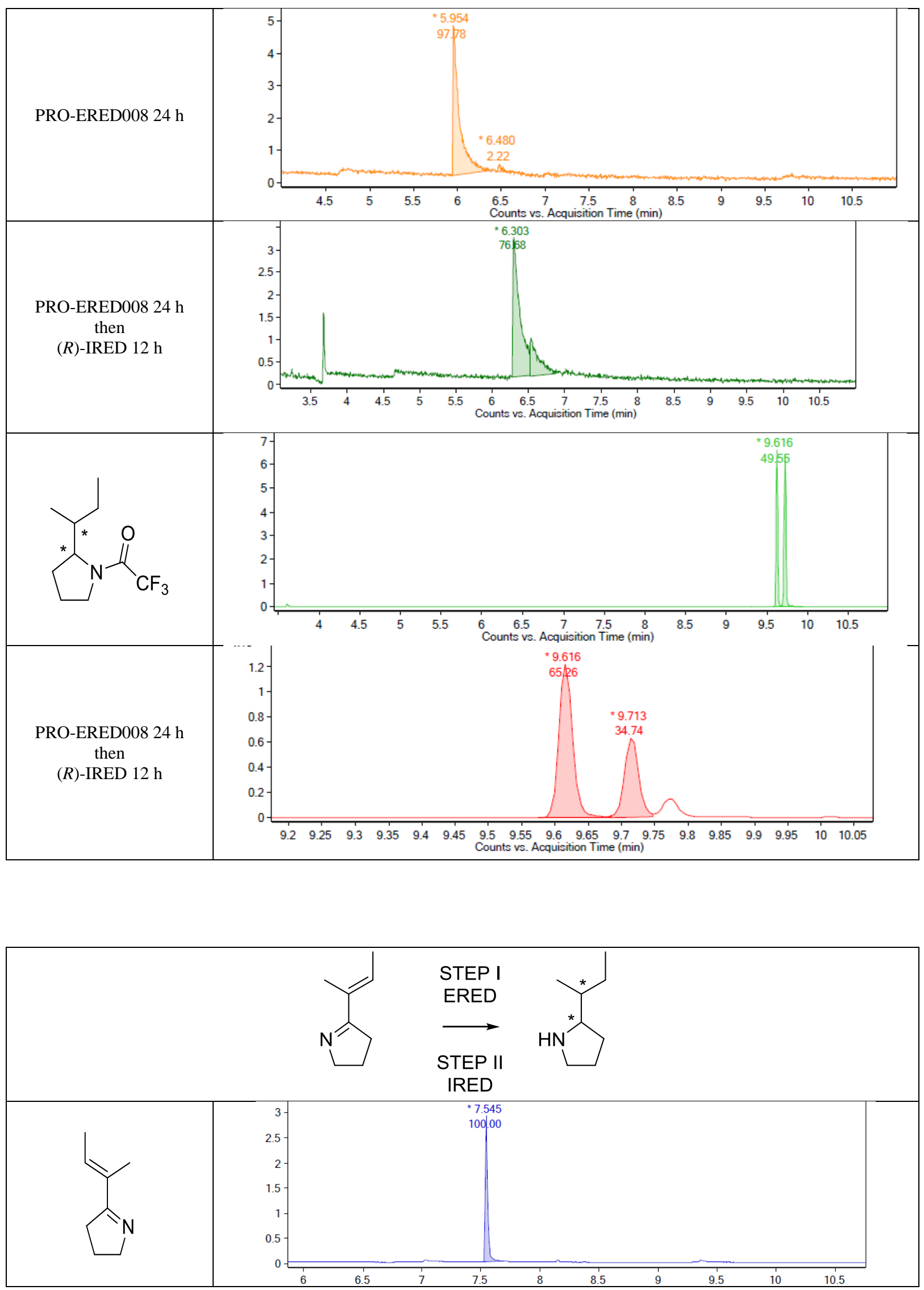


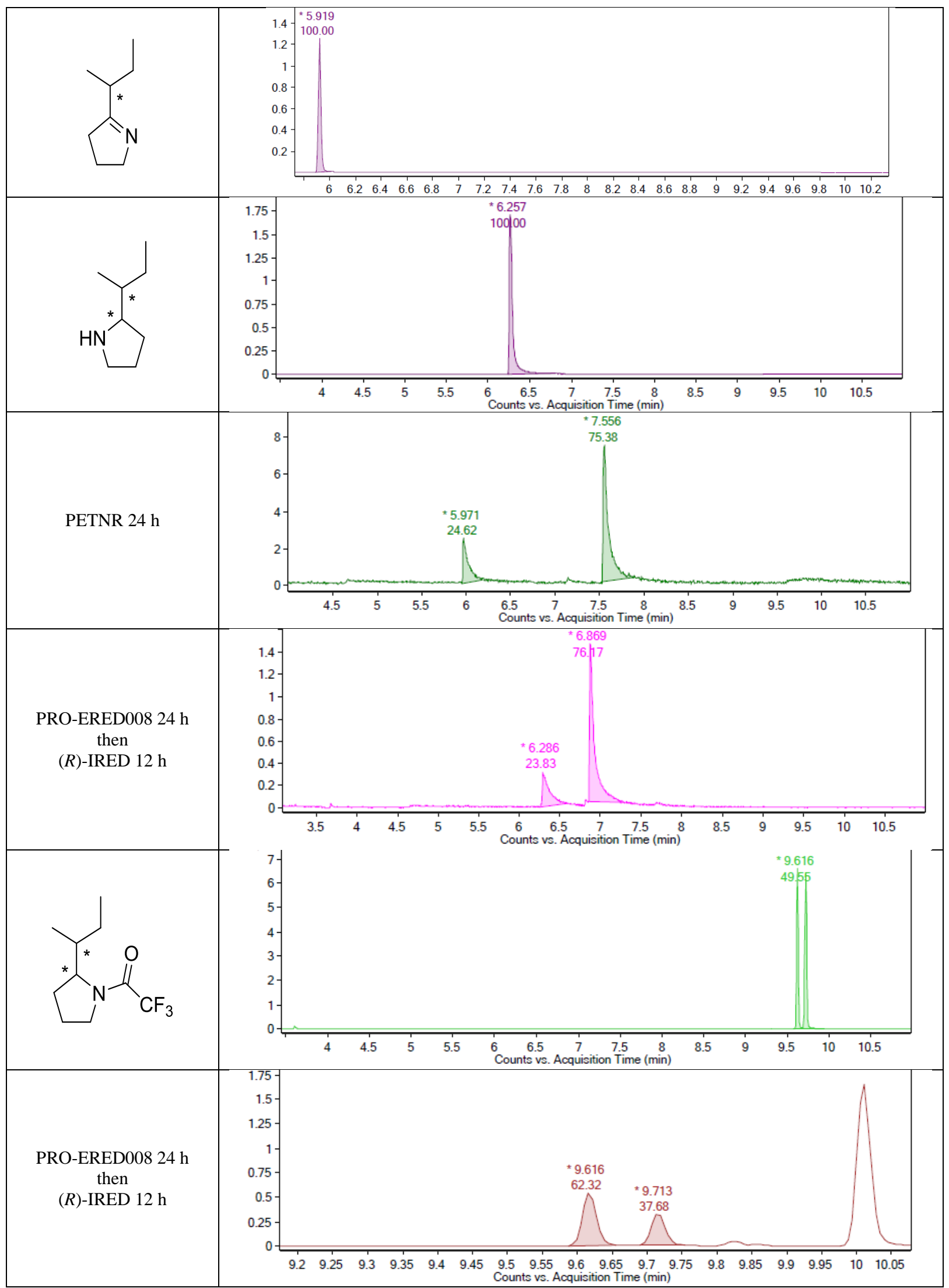




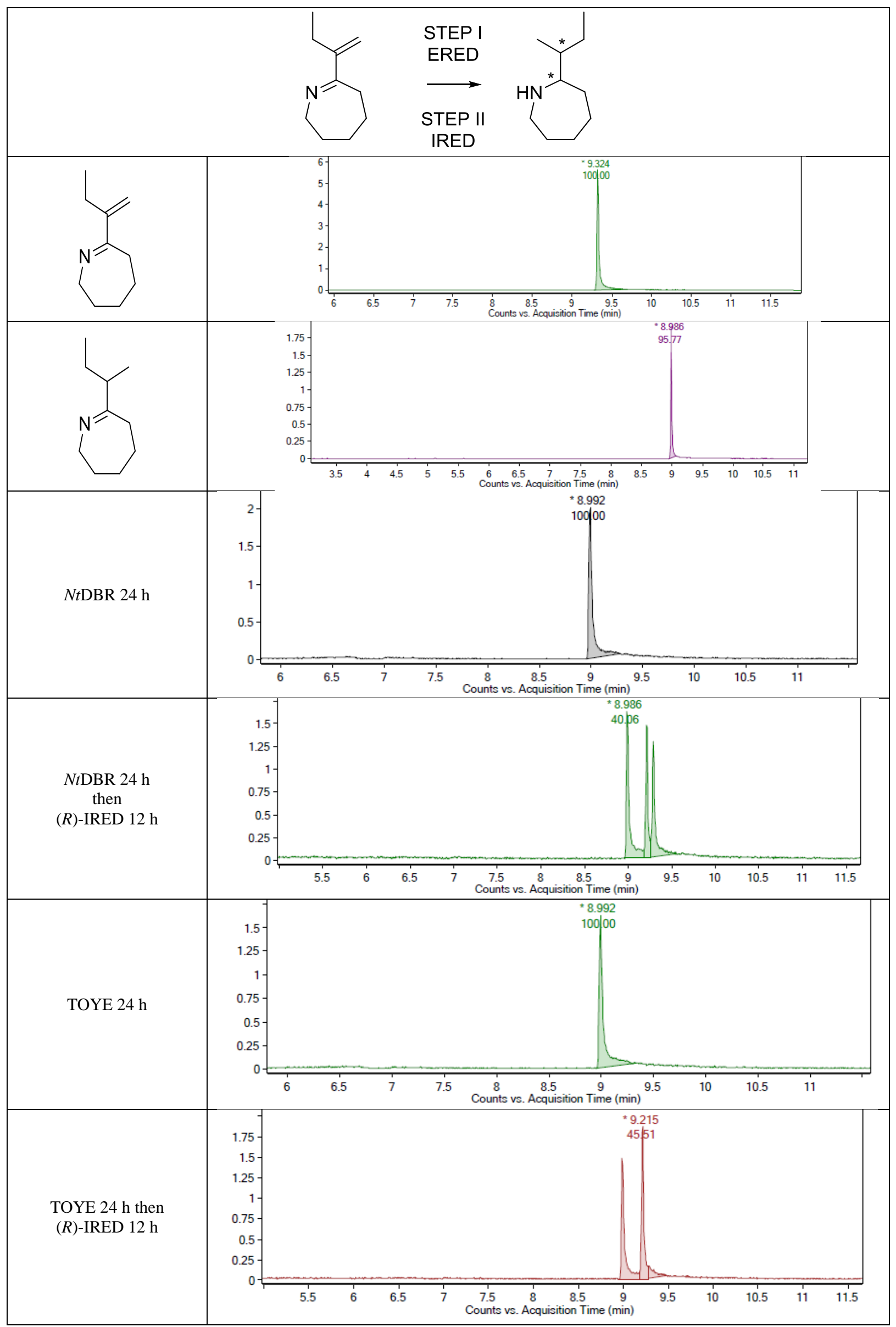



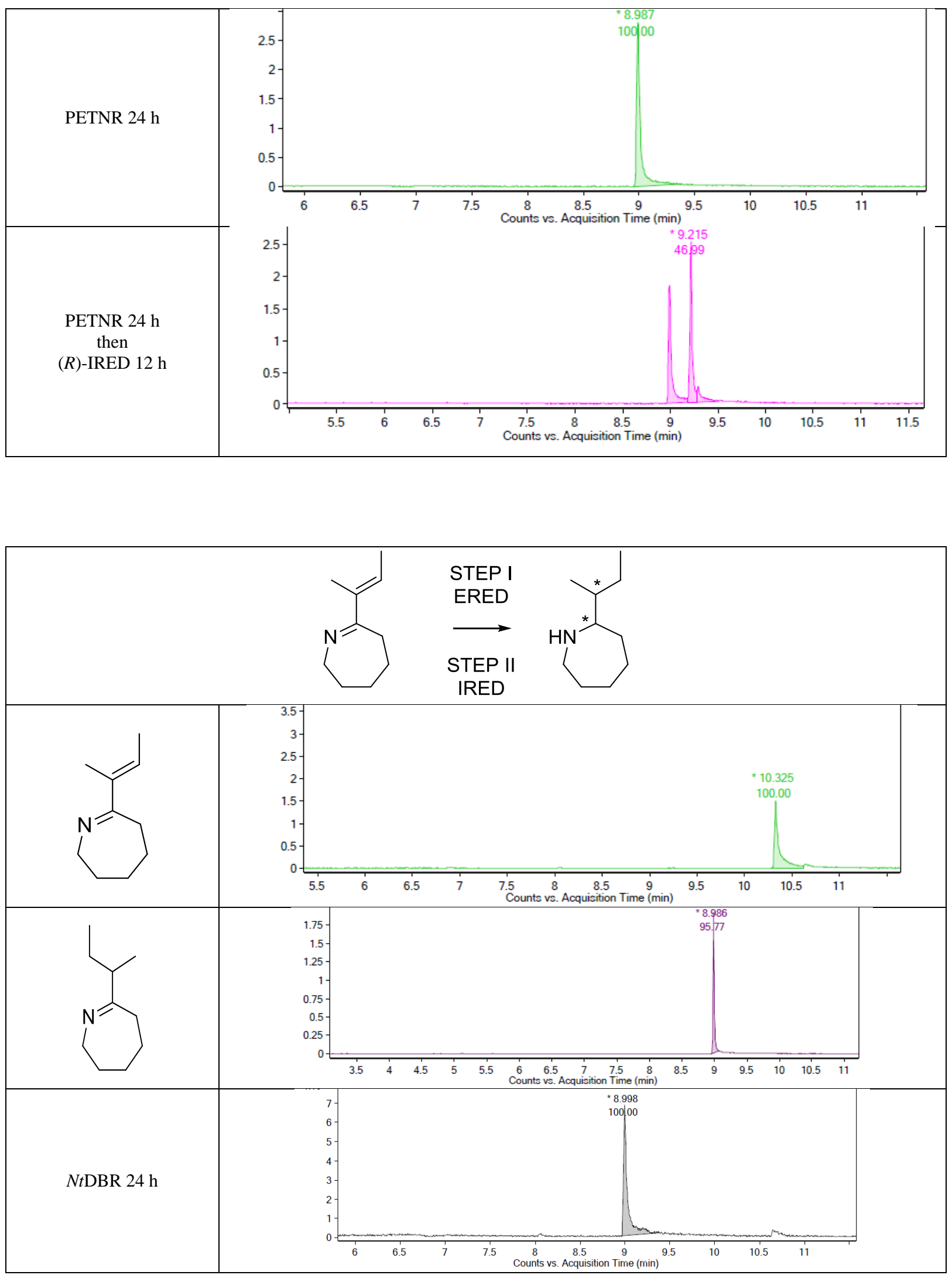


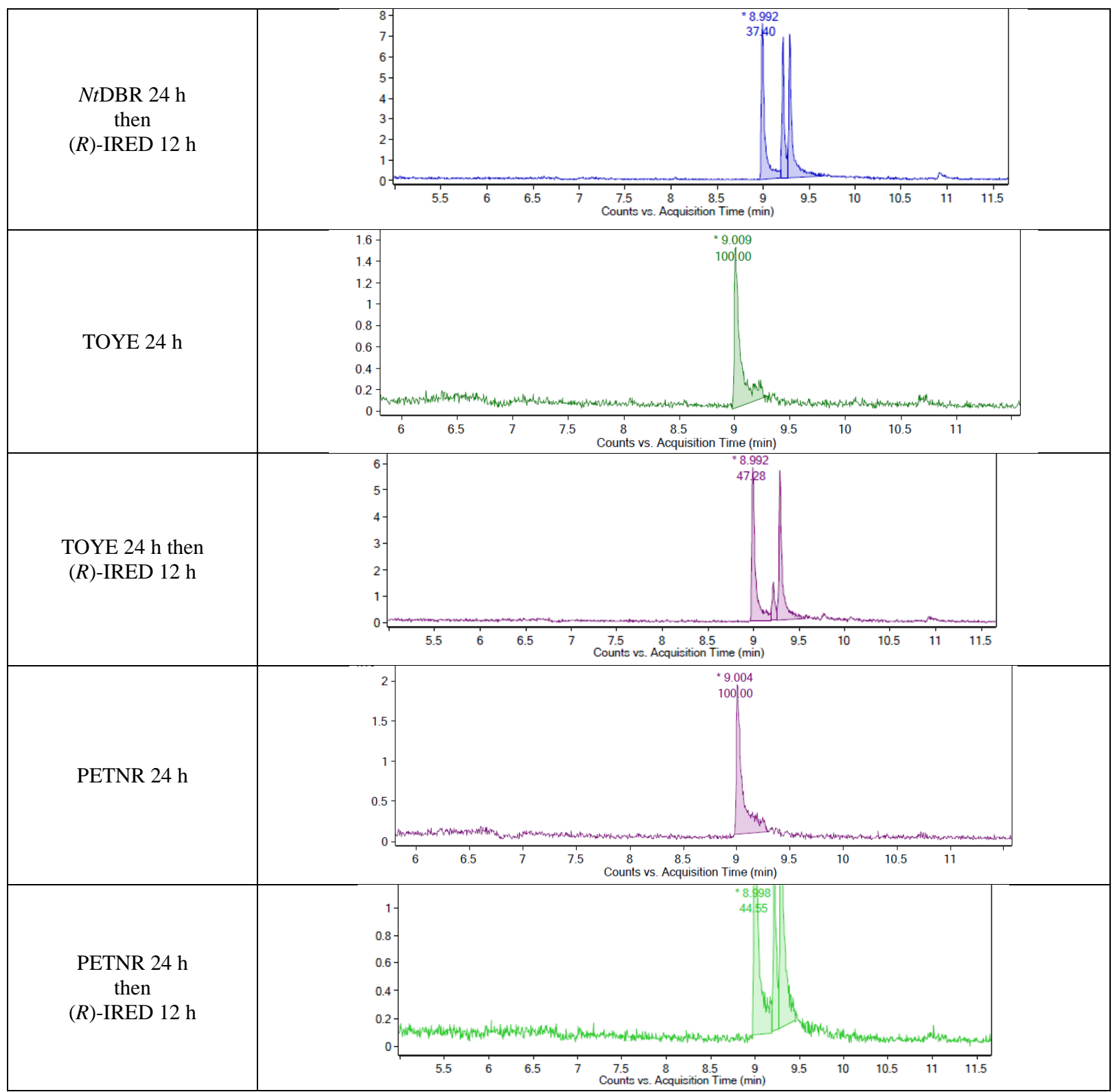


4.2.5 External calibration curve of $17 b$

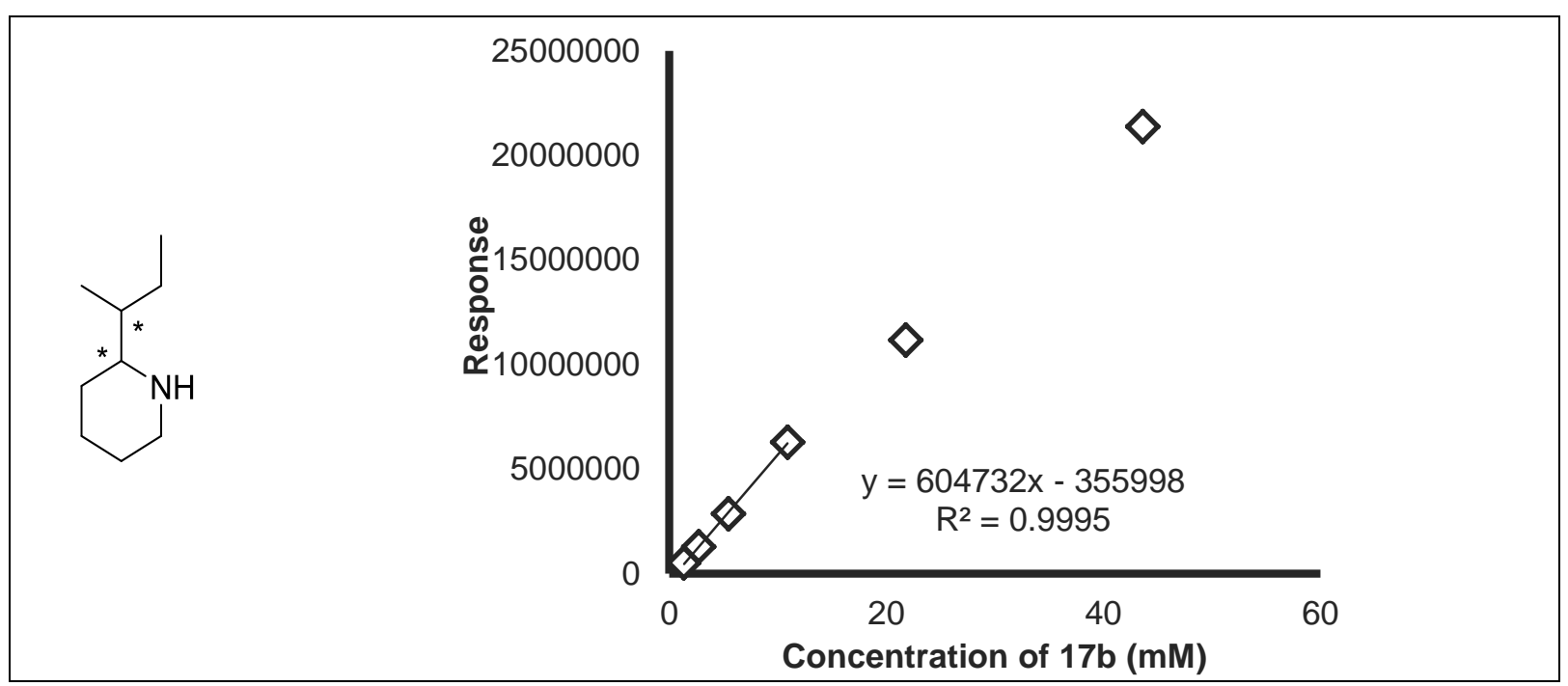




\section{Computational assessment of electrophilicity of ERED substrates}

All relative reactivity calculations were performed using reactivity tools designed in-house at Pfizer that are based on Frontier Molecular Orbital Theory and use a customized workflow. This involved a MCMM/low-mode conformational search to generate the lowest energy conformations in water. Molecular geometries were then optimized at PBE/DND/COSMO-RS level of theory in water using DMol3 software with a $4.4 \AA$ orbital cut-off value. This was followed by a single-point calculation of Fukui indexes $\mathrm{f}_{\mathrm{k}}(+)$ and frontier orbital energies $\left(\varepsilon_{\mathrm{HOMO}}\right.$ and $\left.\varepsilon_{\mathrm{LUMO}}\right)$ at PBE/DNP/COSMORS level of theory in water using a $4.4 \AA$ orbital cut-off and applying a Hirshfeld partition scheme. Settings: solvent $=$ water, 5 conformations, thermal average conformation (*except (Z)-Me 6-membered ring, no conformational changes allowed, therefore simply the straight chain conformation).

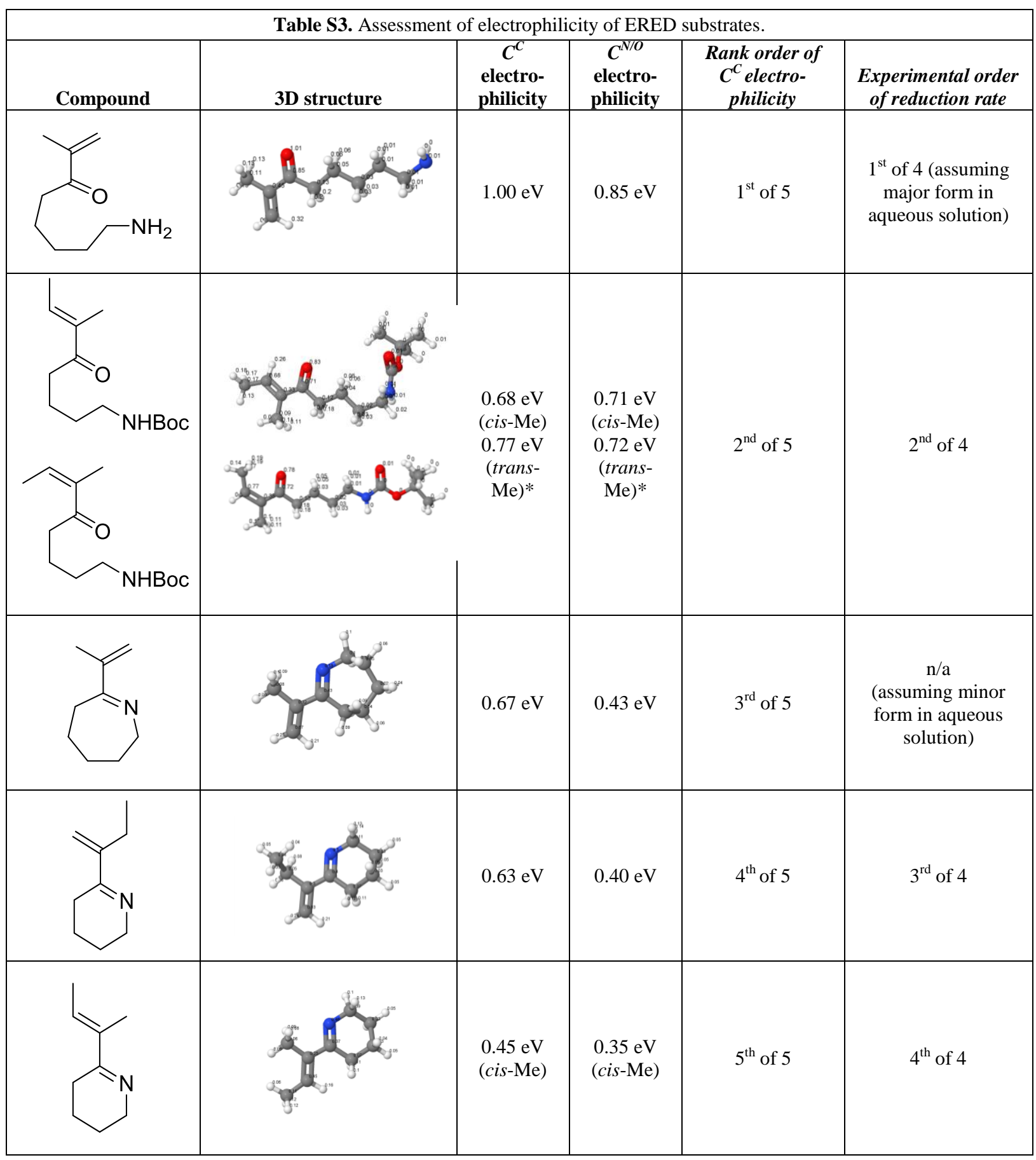

$C^{C}$ refers to the $\beta$-carbon of the $\alpha, \beta$-unsaturated ketone/imine. $C^{N / O}$ refers to the carbon of the ketone/imine. 


\subsection{Isolated product spectra}

\subsection{1 $\quad N$-Boc $\omega$-amino enones}

TTOCT17-83

tert-butyl (5-methyl-4-oxohex-5-en-1-yl)carbamate 1a

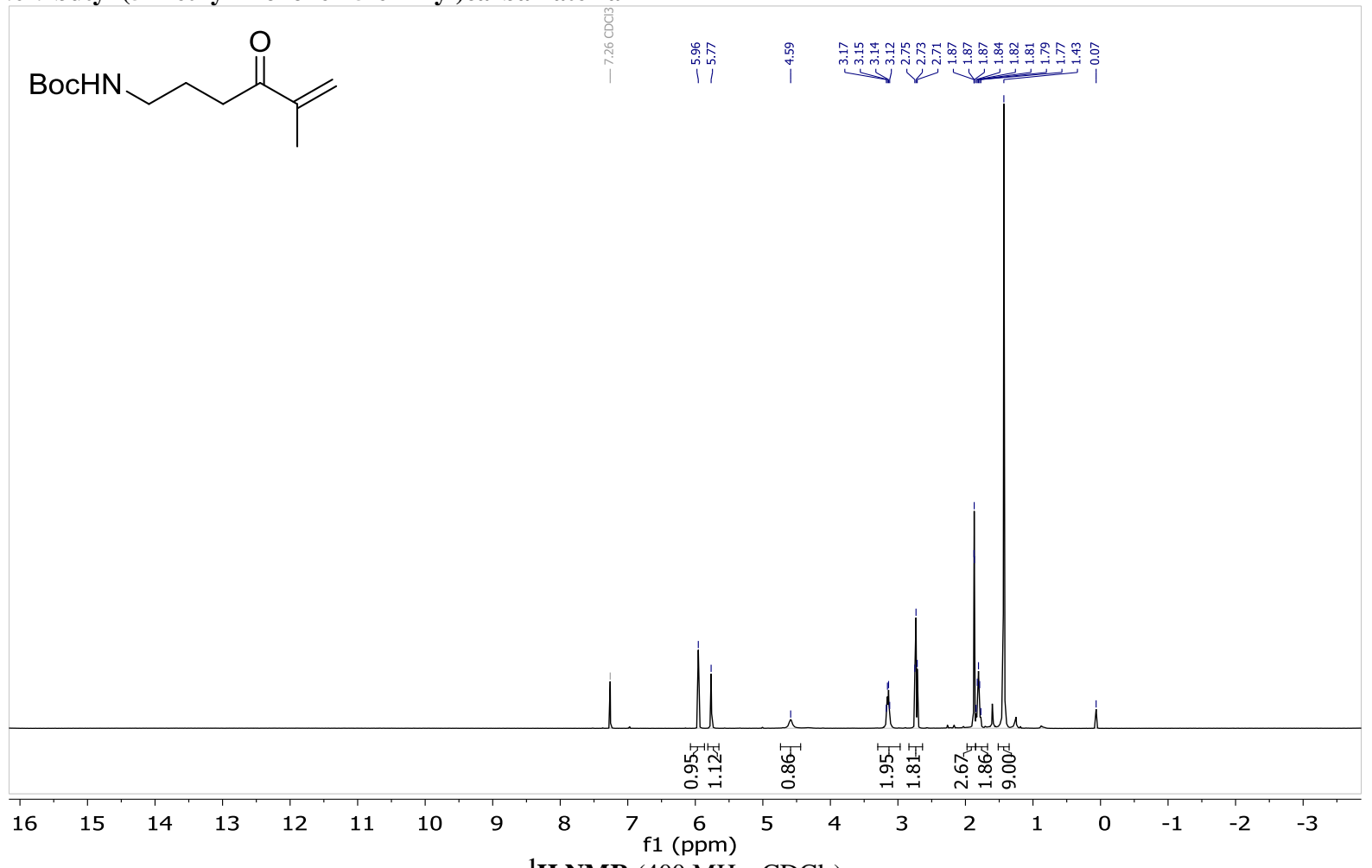

${ }^{1}$ H NMR $\left(400 \mathrm{MHz}, \mathrm{CDCl}_{3}\right)$

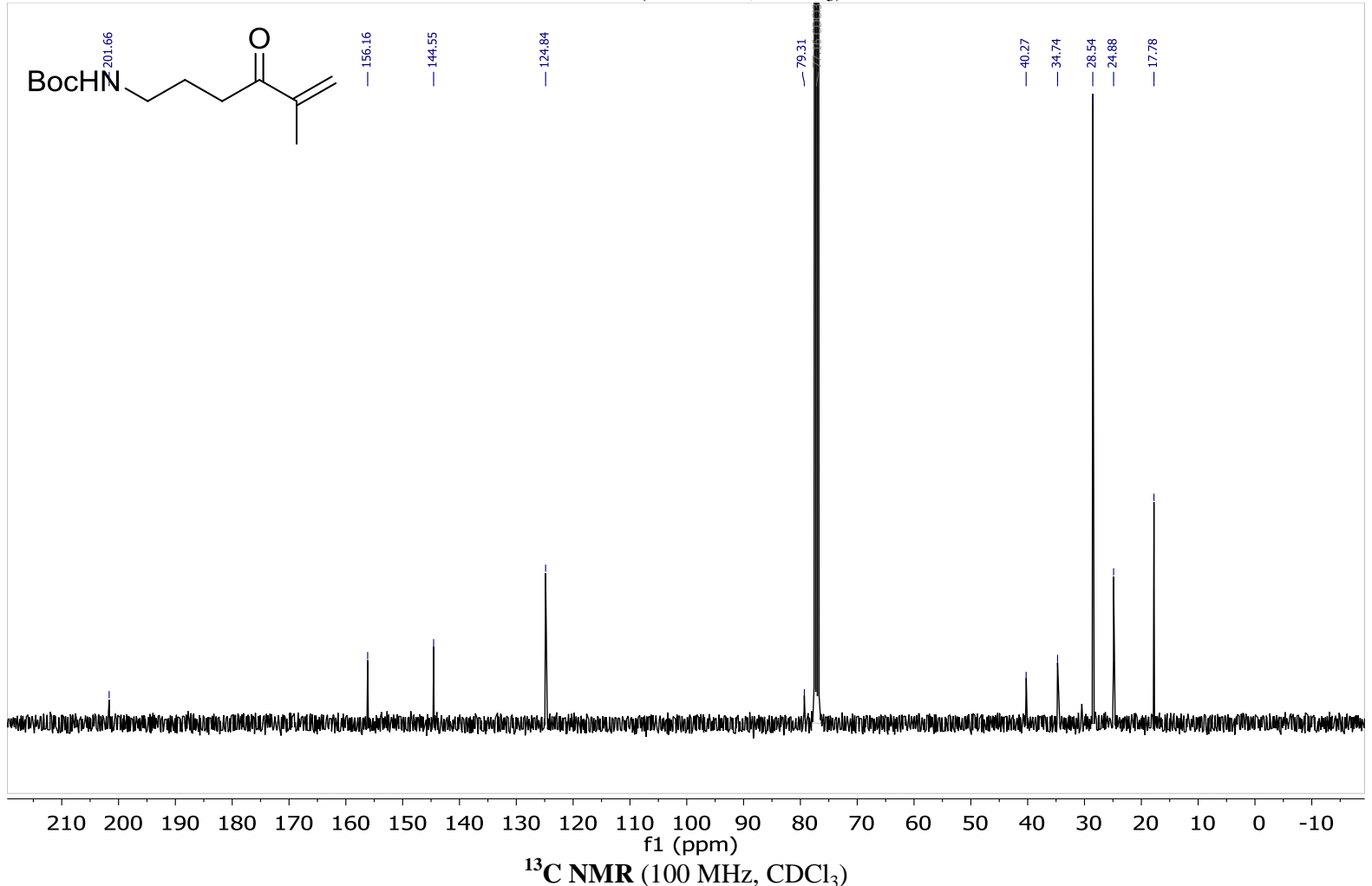


TTOCT17-71

tert-butyl (5-methylene-4-oxoheptyl)carbamate 1b<smiles>C=C(CC)C(=O)CCCNC(=O)O</smiles>

$\underbrace{\infty}$

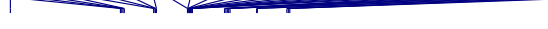

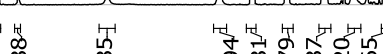

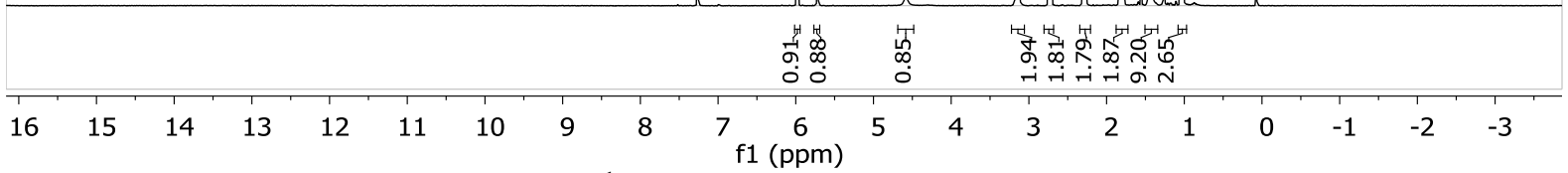

${ }^{1} \mathbf{H}$ NMR $\left(400 \mathrm{MHz}, \mathrm{CDCl}_{3}\right)$

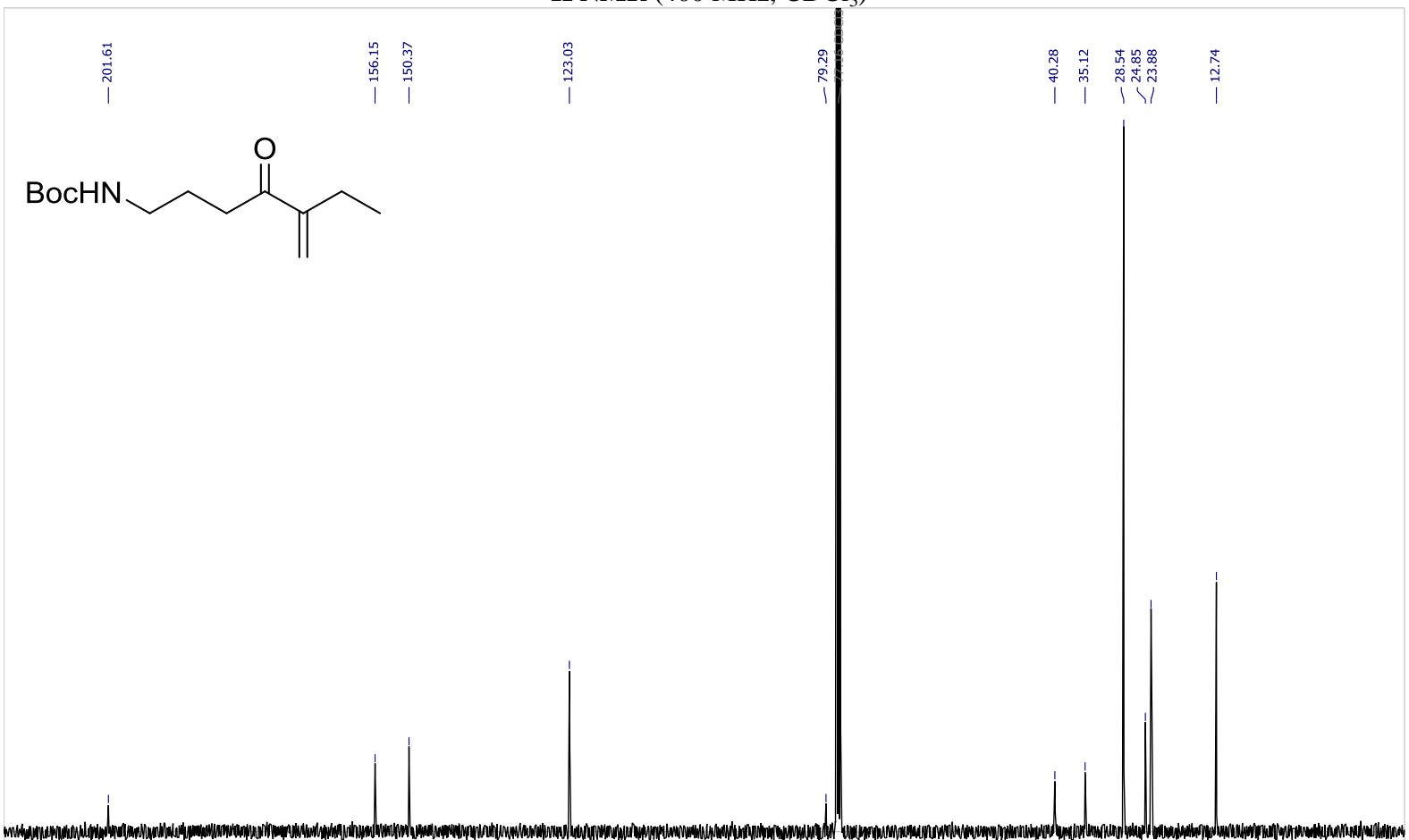

$\begin{array}{lllllllllllllllllllll}210 & 200 & 190 & 180 & 170 & 160 & 150 & 140 & 130 & 120 & \begin{array}{c}110 \\ \mathrm{f} 1\end{array}(\mathrm{ppm}) \\ (\mathrm{ppo}) & 90 & 70 & 60 & 50 & 40 & 30 & 20 & 10 & 0 & -10\end{array}$ ${ }^{13} \mathbf{C}$ NMR $\left(100 \mathrm{MHz}, \mathrm{CDCl}_{3}\right)$ 
TTOCT17-63

tert-butyl (5-methyl-4-oxohept-5-en-1-yl)carbamate 1c

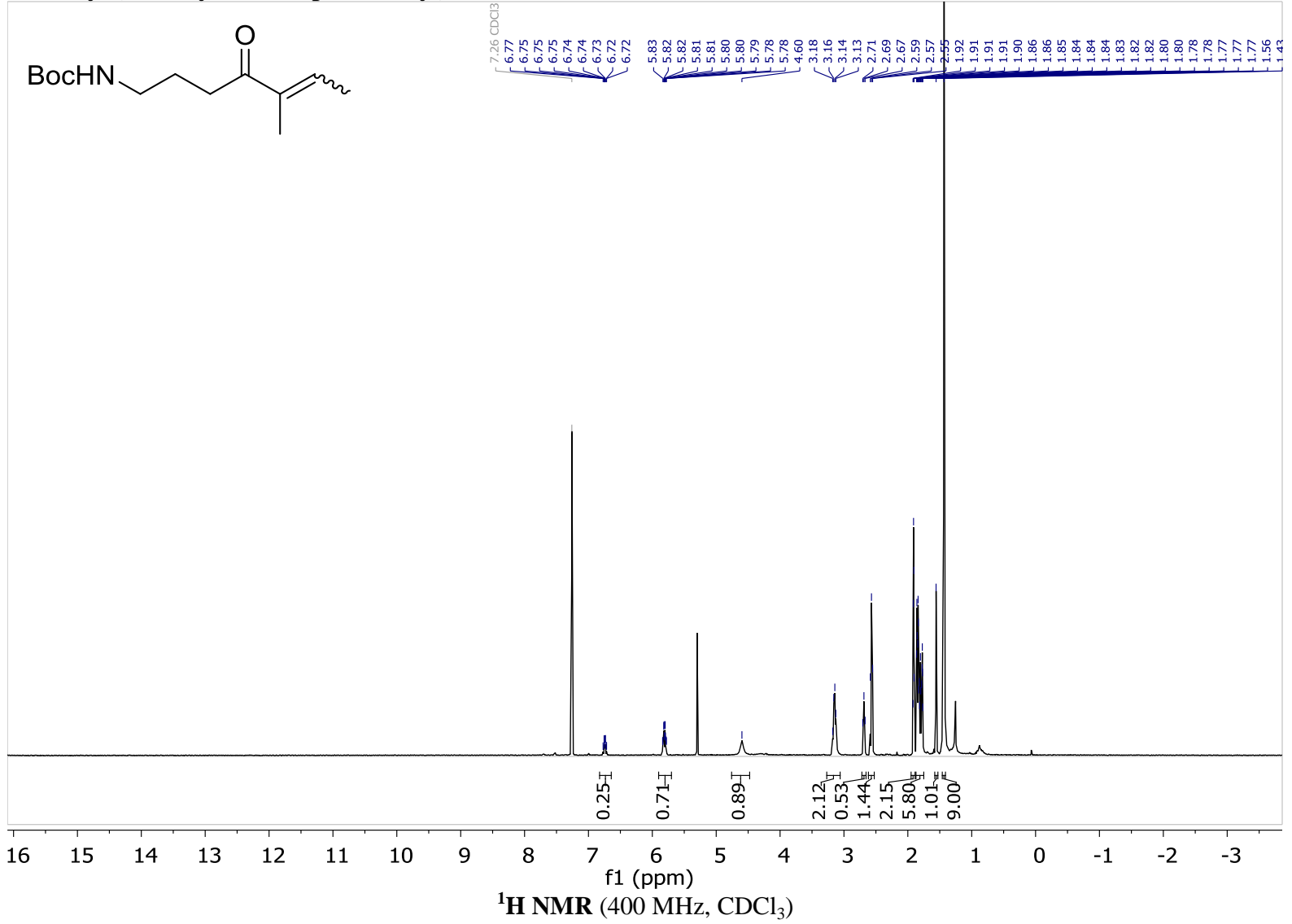


TTOCT17-143

tert-butyl (6-methyl-5-oxohex-6-en-1-yl)carbamate 2a

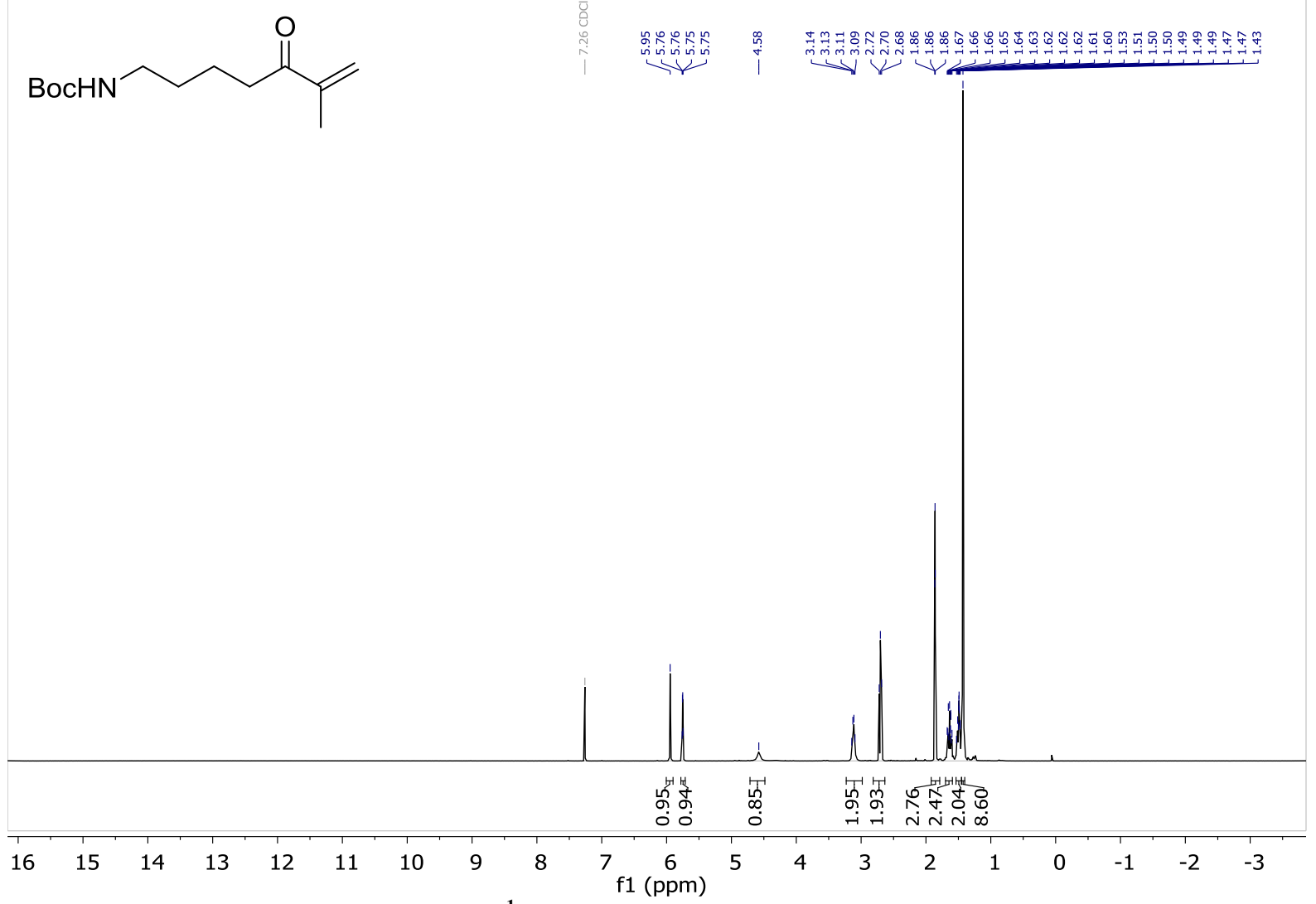

${ }^{1} \mathbf{H}$ NMR $\left(400 \mathrm{MHz}, \mathrm{CDCl}_{3}\right)$

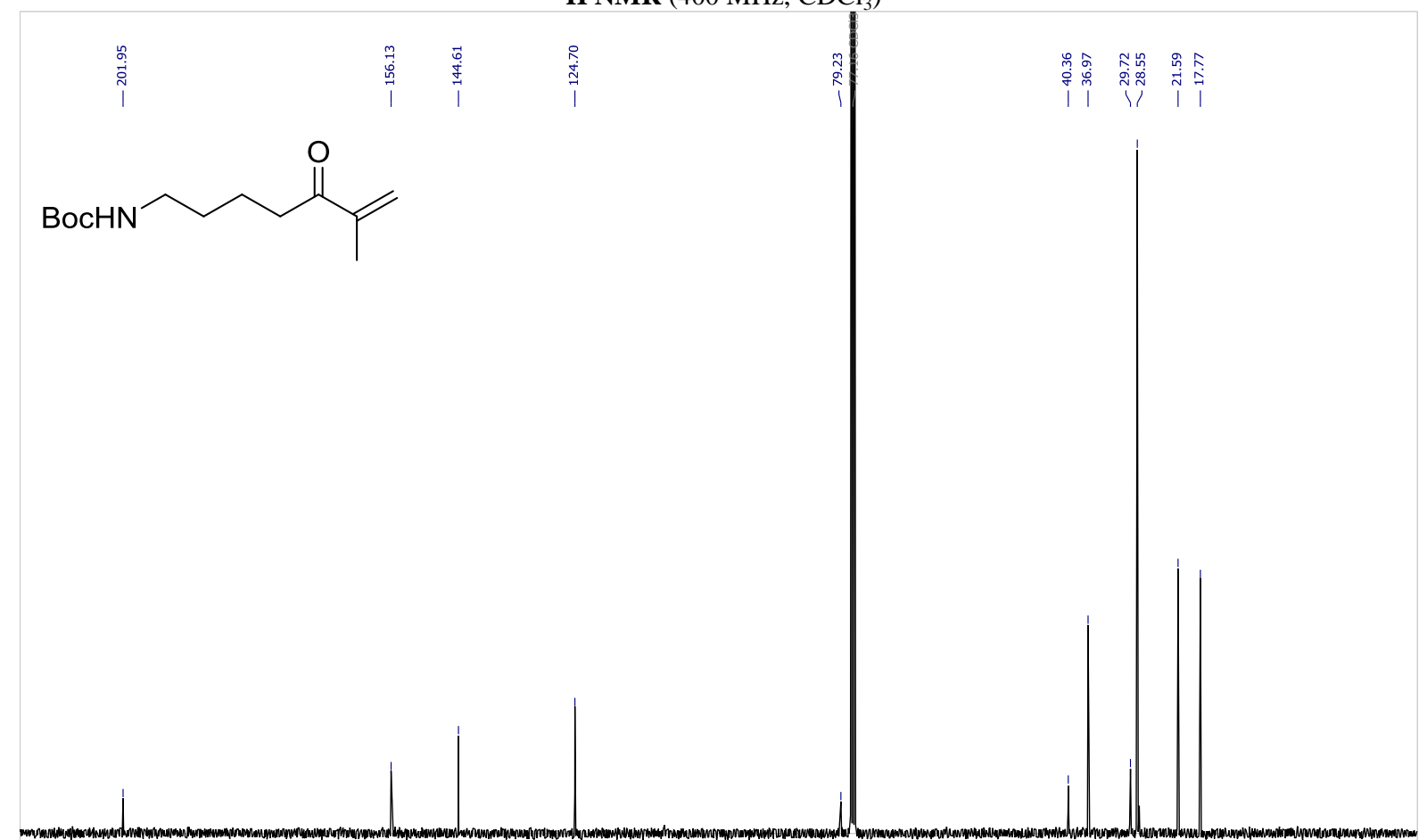

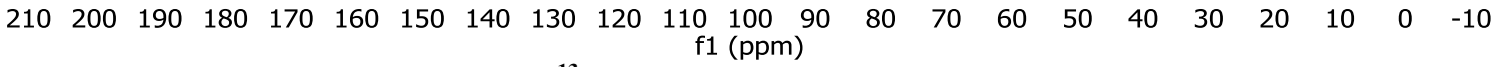

${ }^{13} \mathrm{C}$ NMR $\left(100 \mathrm{MHz}, \mathrm{CDCl}_{3}\right)$ 
TTOCT17-58

tert-butyl (6-methyl-5-oxooct-6-en-1-yl)carbamate 2c

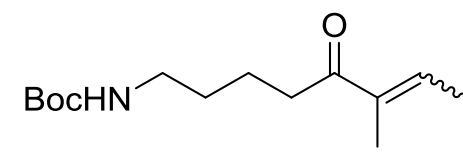

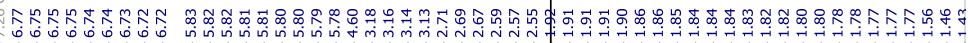

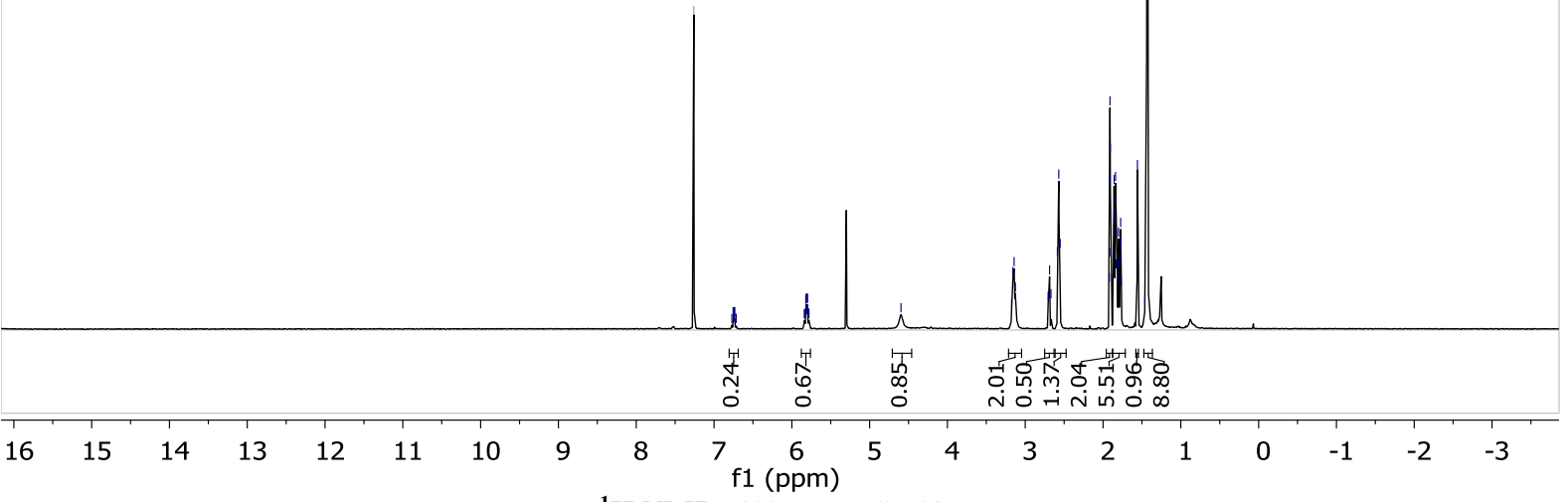

${ }^{1} \mathbf{H}$ NMR $\left(400 \mathrm{MHz}, \mathrm{CDCl}_{3}\right)$ 
TTOCT17-145

tert-butyl (7-methyl-6-oxooct-7-en-1-yl)carbamate 3a
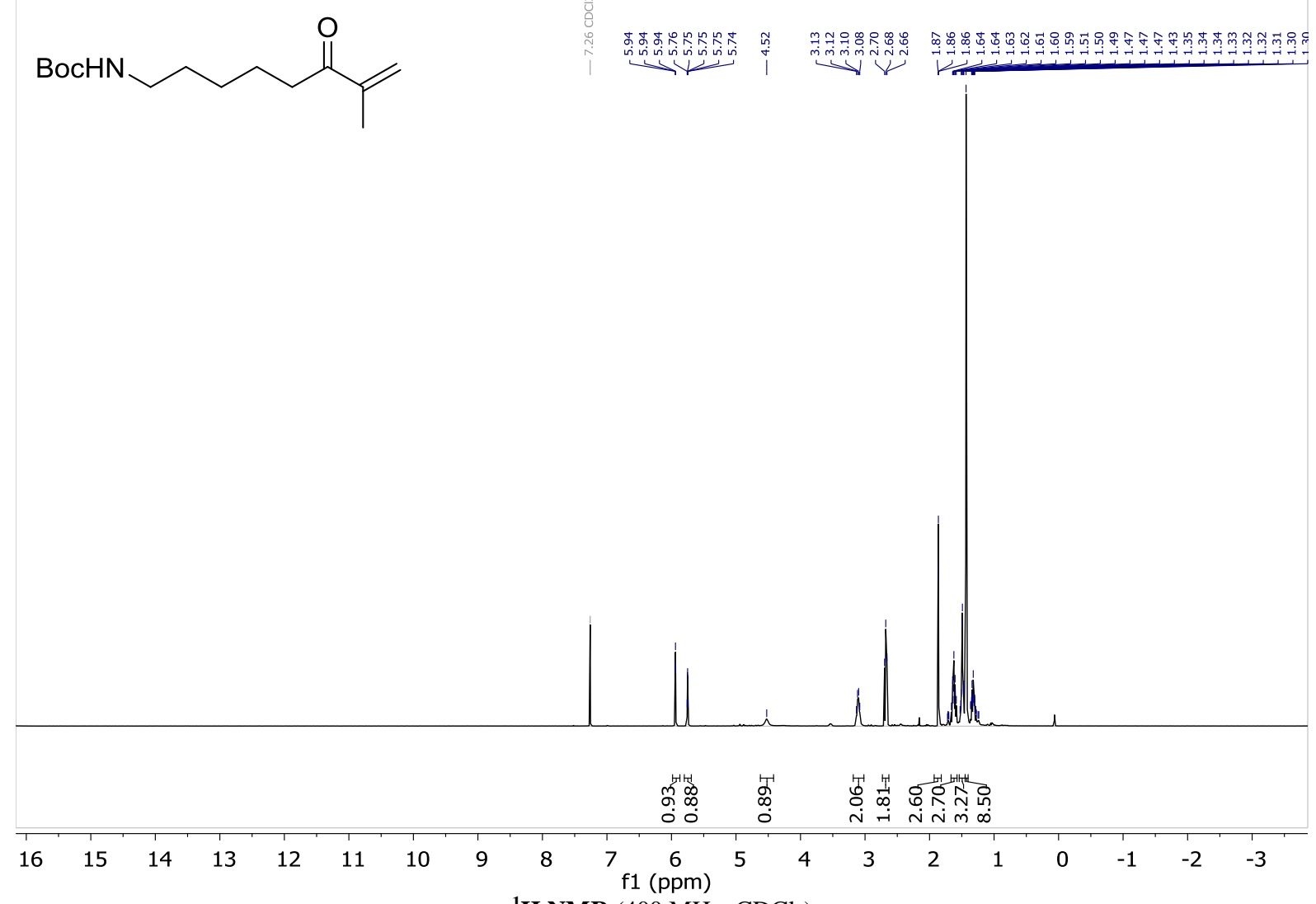

${ }^{1} \mathbf{H}$ NMR $\left(400 \mathrm{MHz}, \mathrm{CDCl}_{3}\right)$

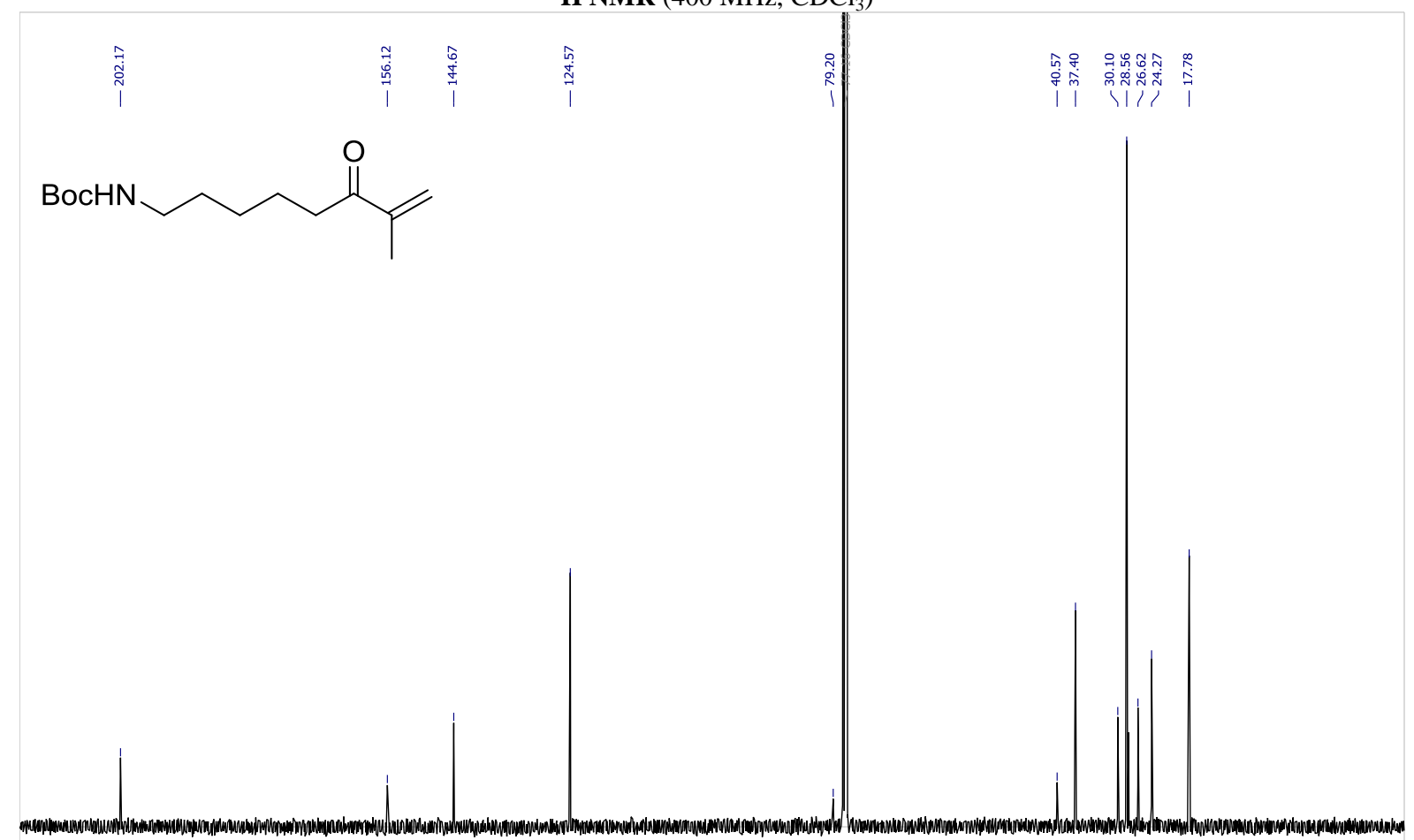

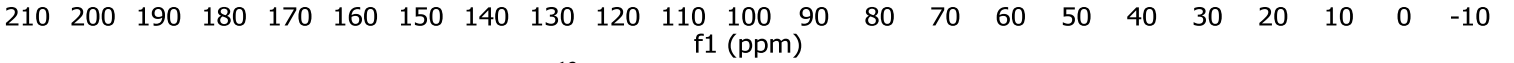
${ }^{13} \mathrm{C}$ NMR $\left(100 \mathrm{MHz}, \mathrm{CDCl}_{3}\right)$ 
TTOCT17-145

tert-butyl (7-methylene-6-oxononyl)carbamate 3b

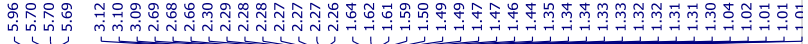

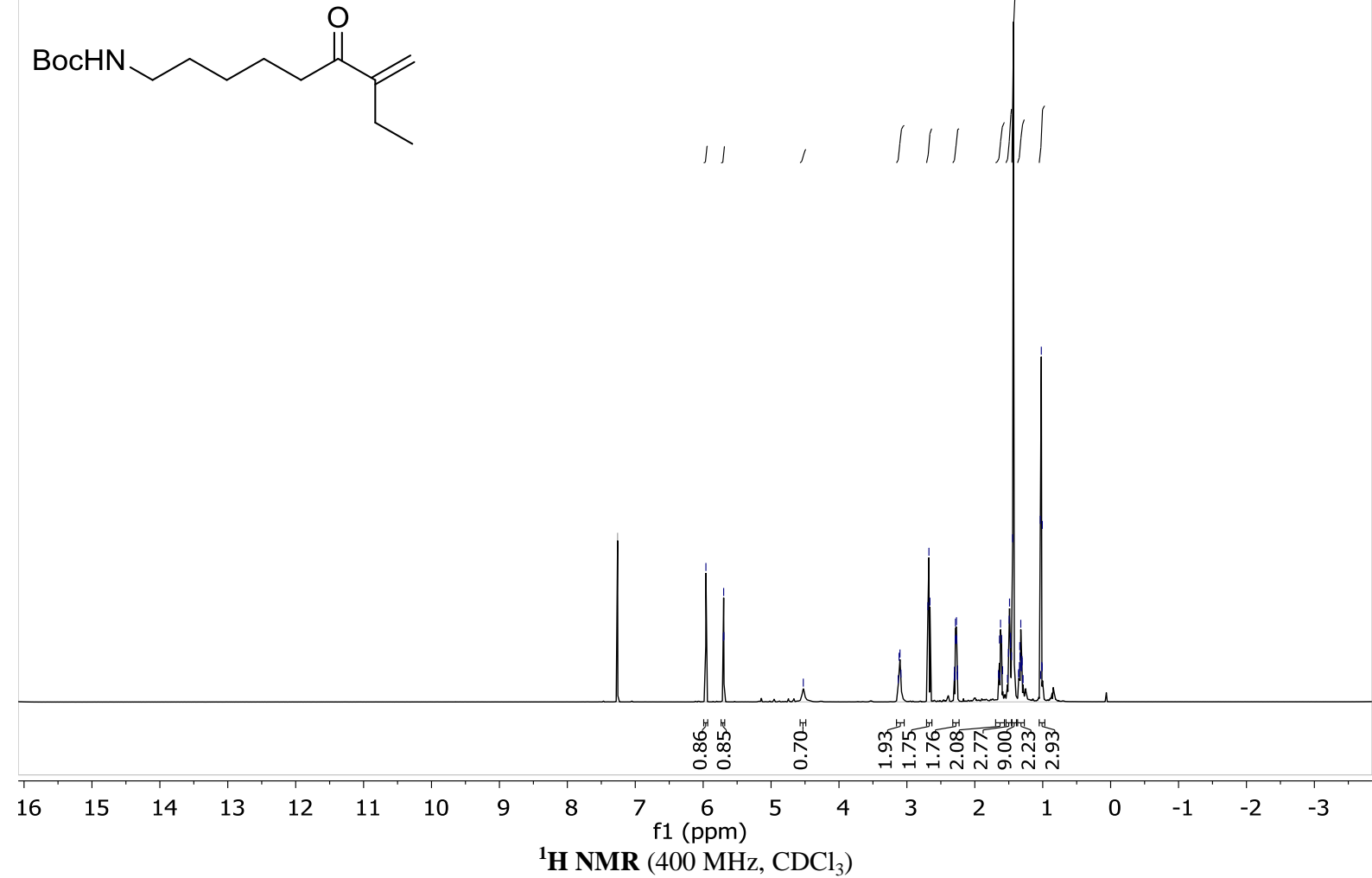


TTAUG18-112-3

tert-butyl (7-methyl-6-oxonon-7-en-1-yl)carbamate 3c

$\frac{20}{8}$

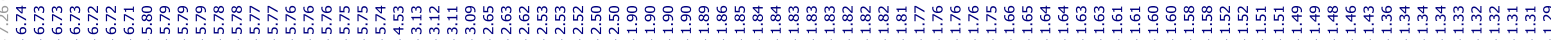<smiles>CC=C(C)C(=O)CCCCCNC(=O)O</smiles>

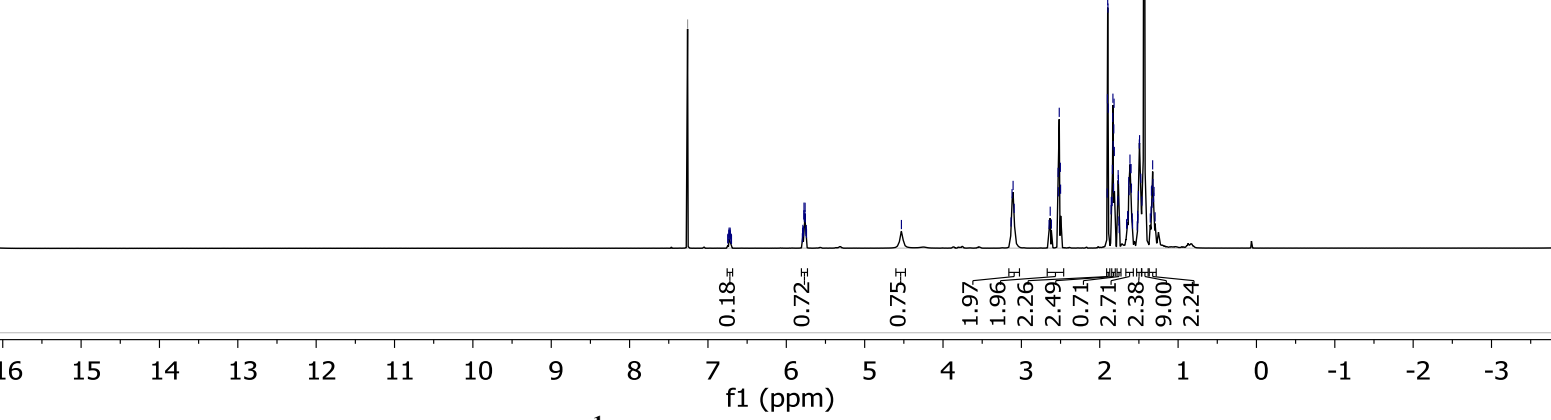

${ }^{1} \mathbf{H}$ NMR (400 MHz, $\mathrm{CDCl}_{3}$ ) 
6.1.2 $\quad N$-Boc $\omega$-amino ketones

TTOCT17-106

tert-butyl (5-methyl-4-oxohexyl)carbamate 10a

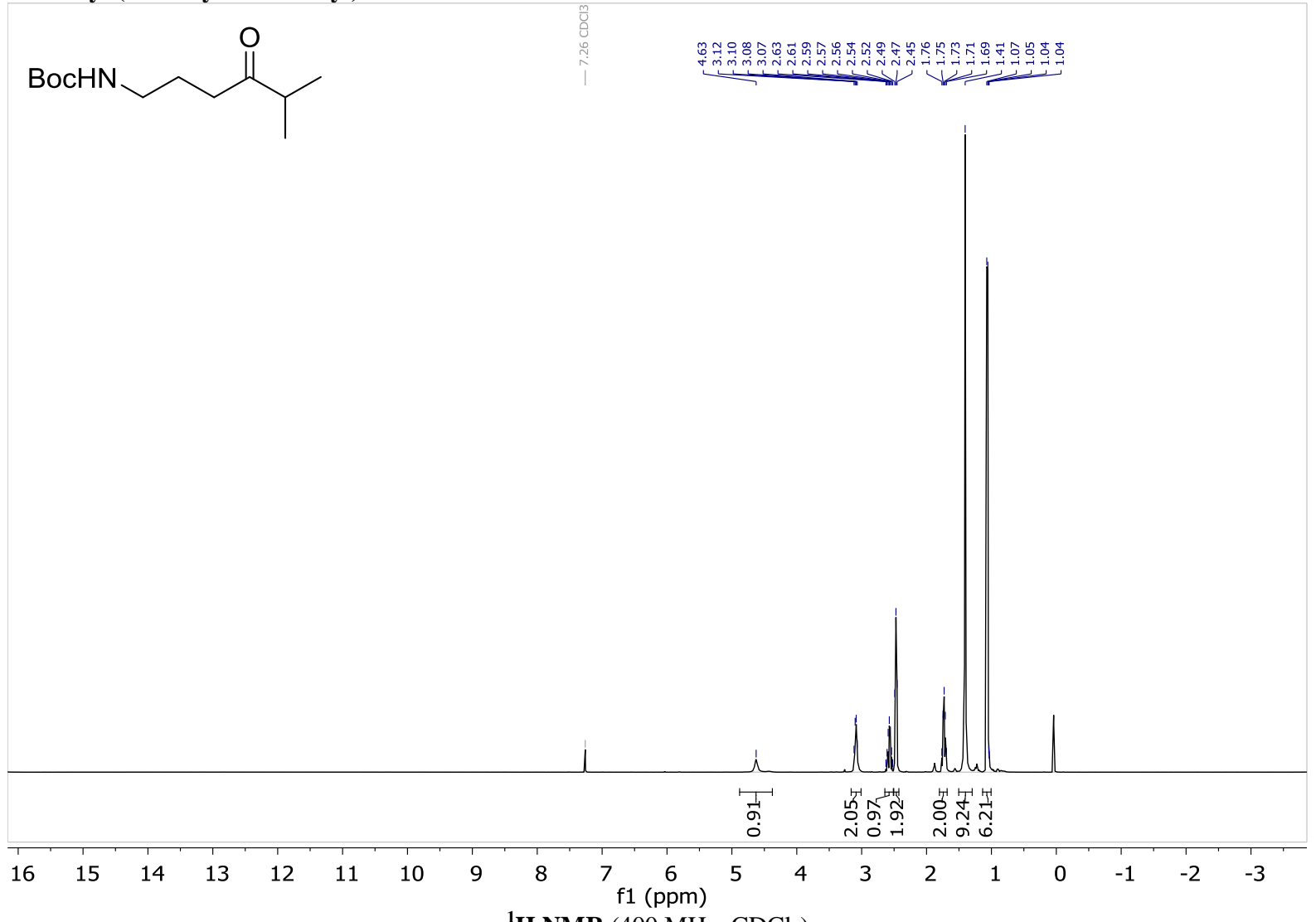

${ }^{1} \mathbf{H}$ NMR $\left(400 \mathrm{MHz}, \mathrm{CDCl}_{3}\right)$

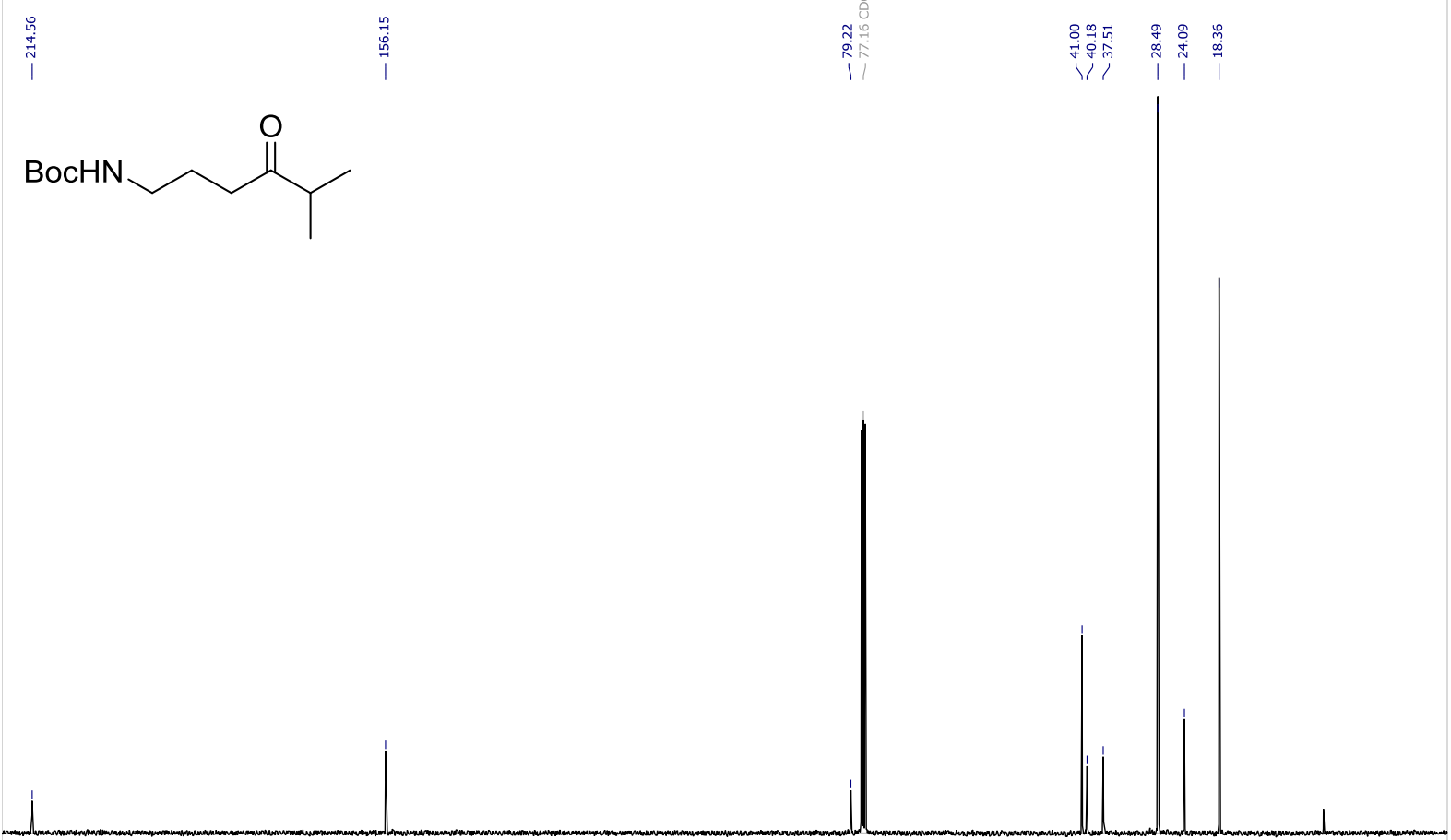

$\begin{array}{lllllllllllllllllllllll}210 & 200 & 190 & 180 & 170 & 160 & 150 & 140 & 130 & 120 & 110 & 100 & 90 & 80 & 70 & 60 & 50 & 40 & 30 & 20 & 10 & 0 & -10\end{array}$ ${ }^{13} \mathrm{C}$ NMR $\left(100 \mathrm{MHz}, \mathrm{CDCl}_{3}\right)$ 
TTOCT17-89

tert-butyl (6-methyl-5-oxoheptyl)carbamate 11a

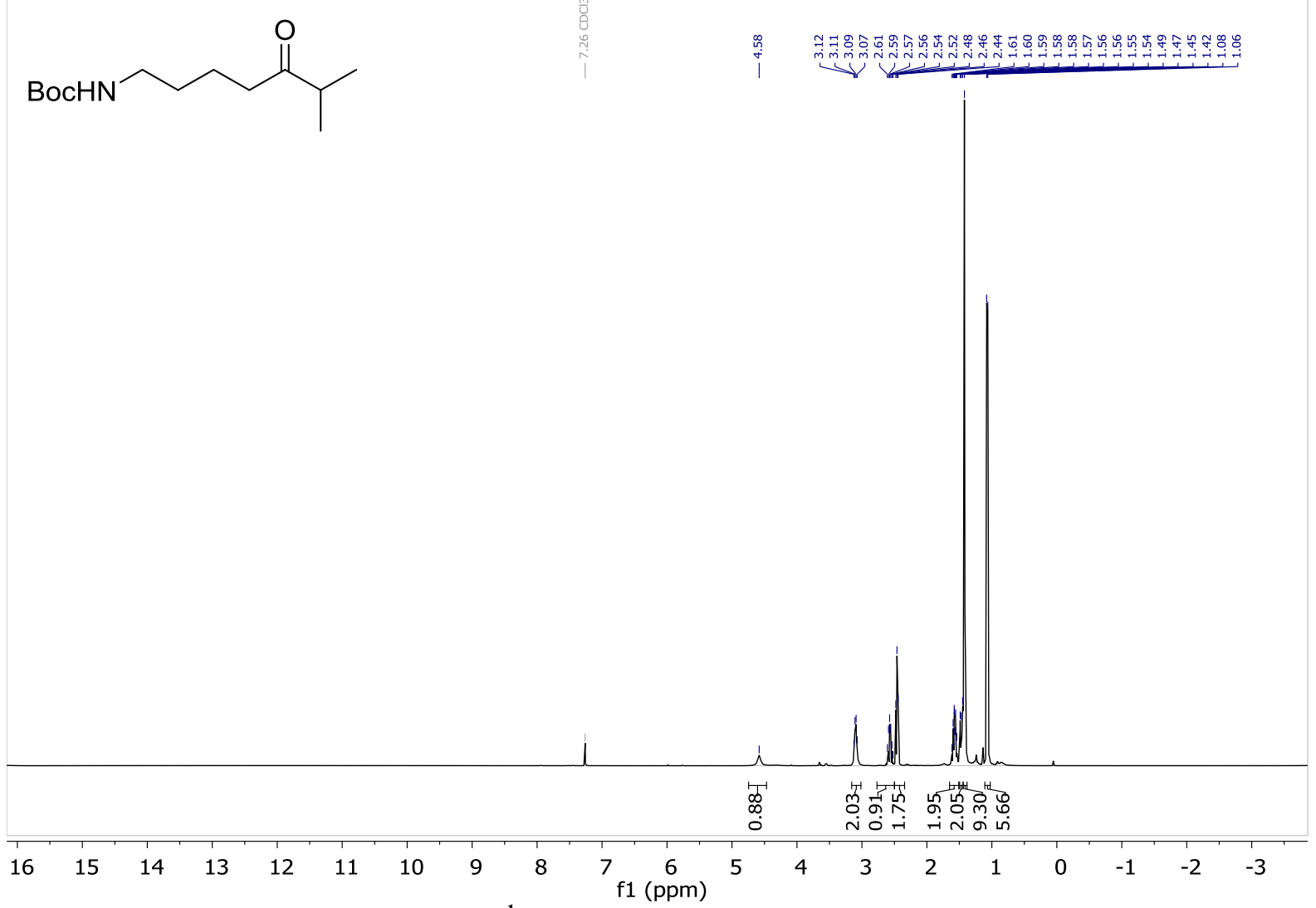

${ }^{1} \mathbf{H}$ NMR $\left(400 \mathrm{MHz}, \mathrm{CDCl}_{3}\right)$<smiles>CC(C)C(=O)CCCCNC(=O)O</smiles>
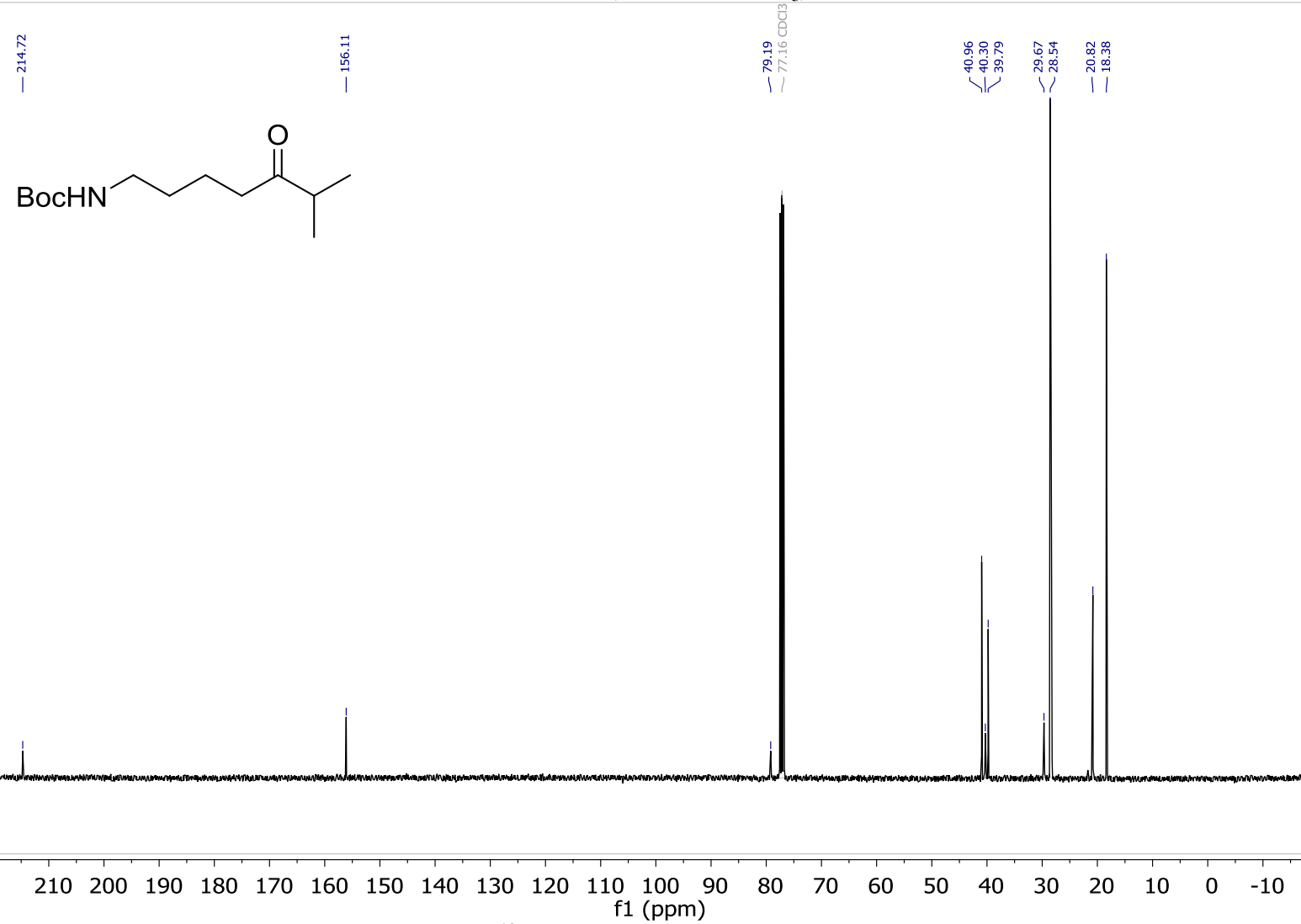

${ }^{13} \mathbf{C}$ NMR (100 MHz, $\left.\mathrm{CDCl}_{3}\right)$ 
TTOCT17-132

tert-butyl-(6-methyl-5-oxooctyl)carbamate 11b

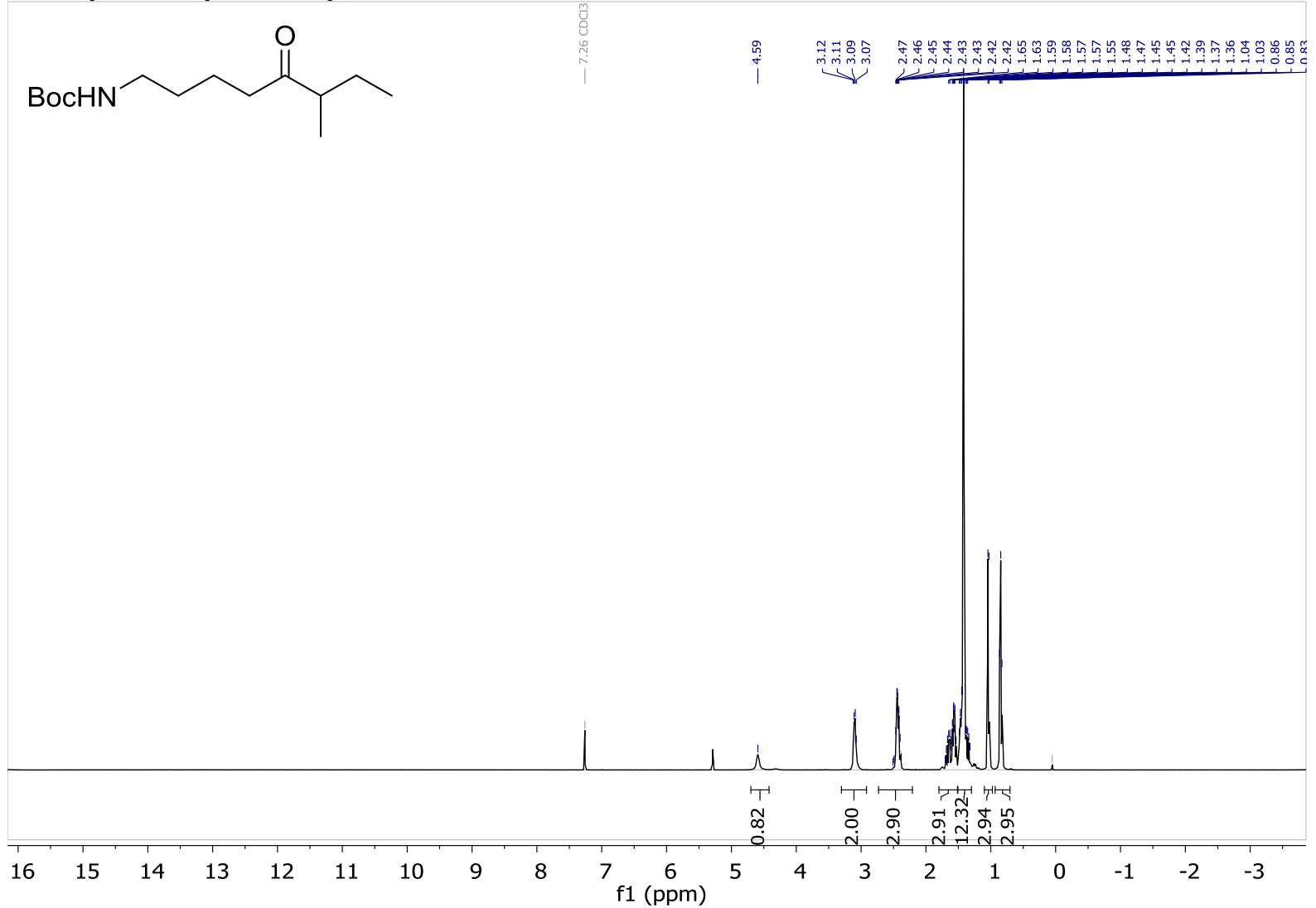

${ }^{1} \mathbf{H}$ NMR $\left(400 \mathrm{MHz}, \mathrm{CDCl}_{3}\right)$
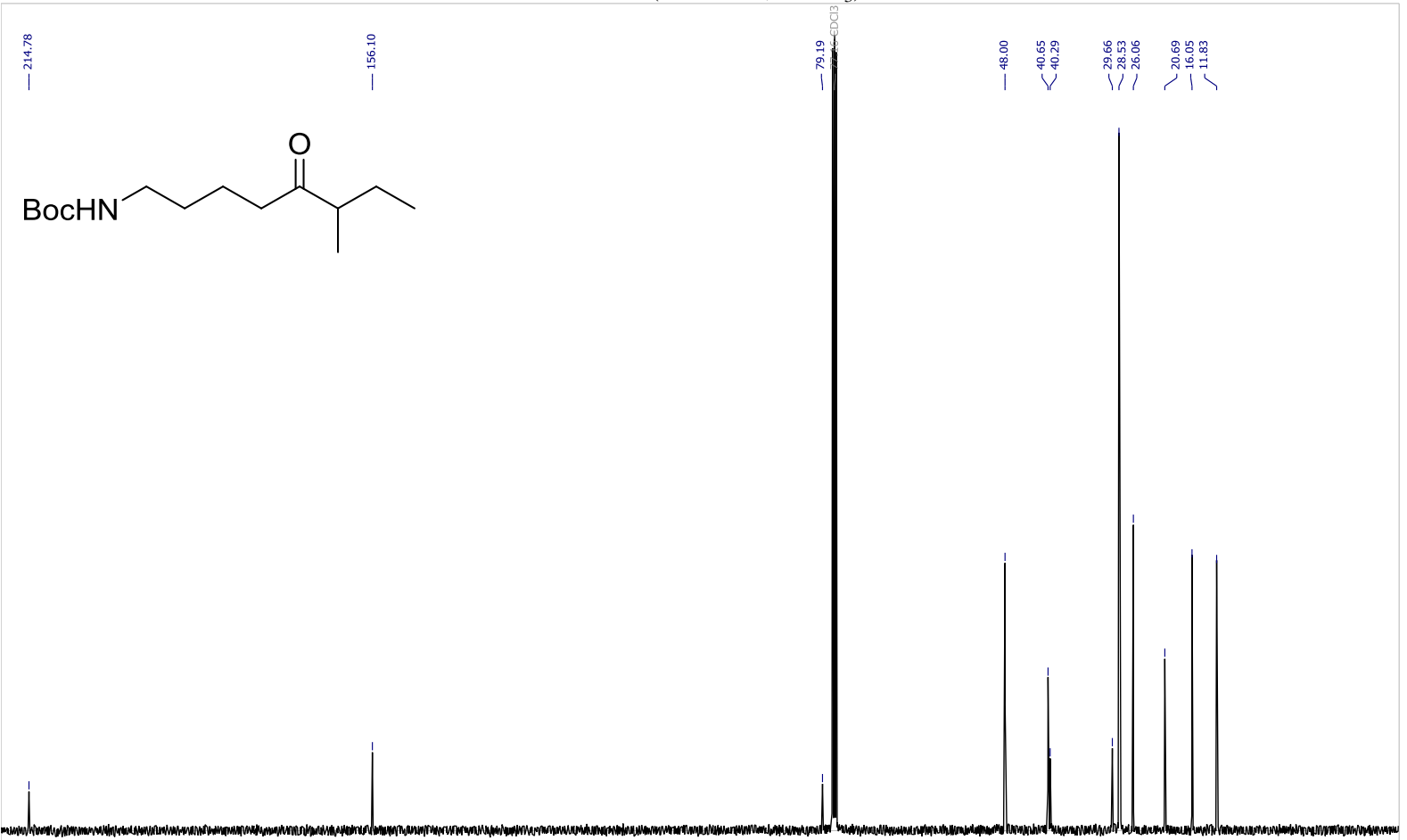

$\begin{array}{lllllllllllllllllllllll}210 & 200 & 190 & 180 & 170 & 160 & 150 & 140 & 130 & 120 & 110 \underset{\mathrm{f} 1(\mathrm{ppm})}{100} & 90 & 80 & 70 & 60 & 50 & 40 & 30 & 20 & 10 & 0 & -10\end{array}$ ${ }^{13}$ C NMR $\left(100 \mathrm{MHz}, \mathrm{CDCl}_{3}\right)$ 
TTOCT17-157

tert-butyl-(6-methyl-5-oxooctyl)carbamate 11b

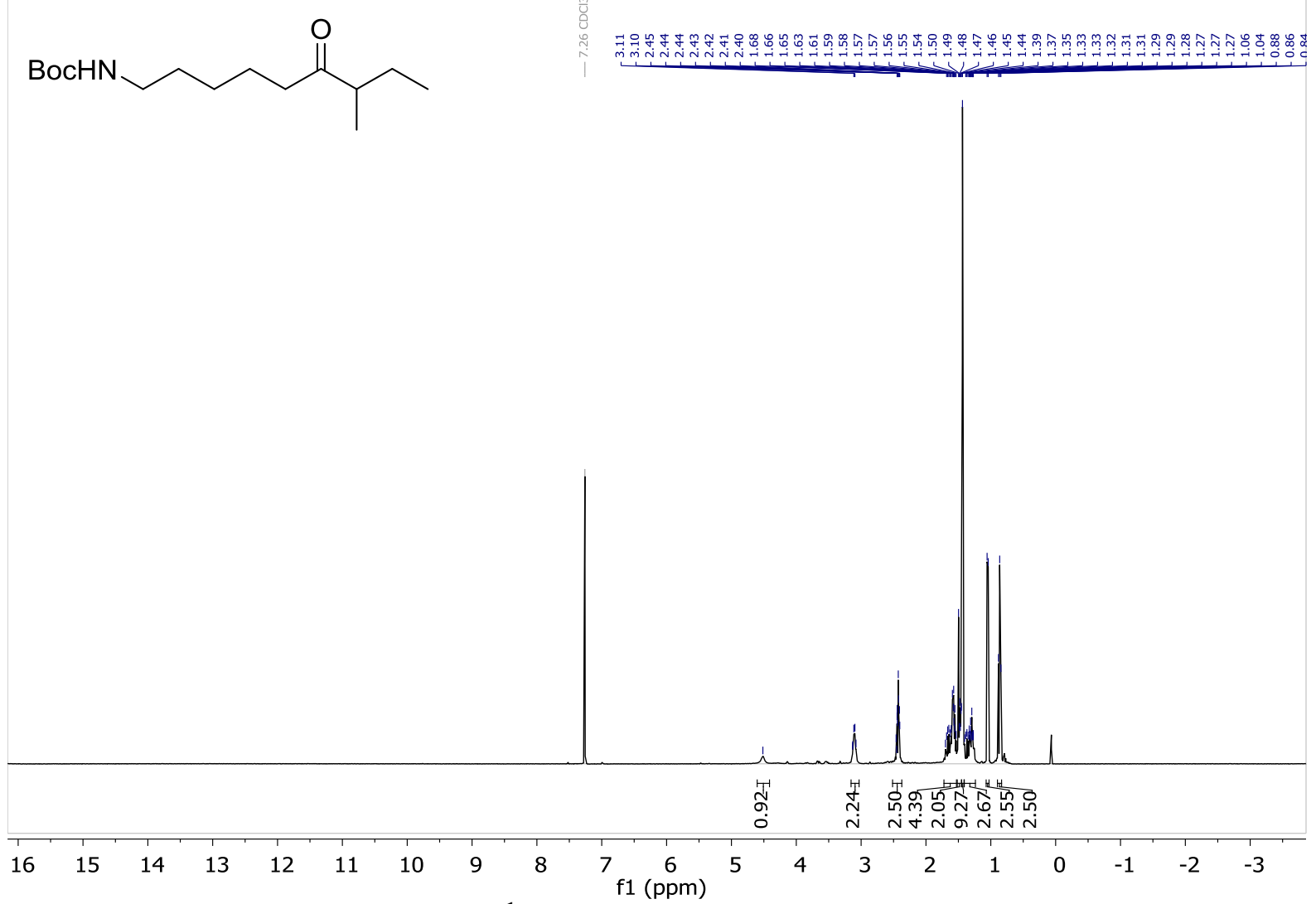

${ }^{1}$ H NMR $\left(400 \mathrm{MHz}, \mathrm{CDCl}_{3}\right)$

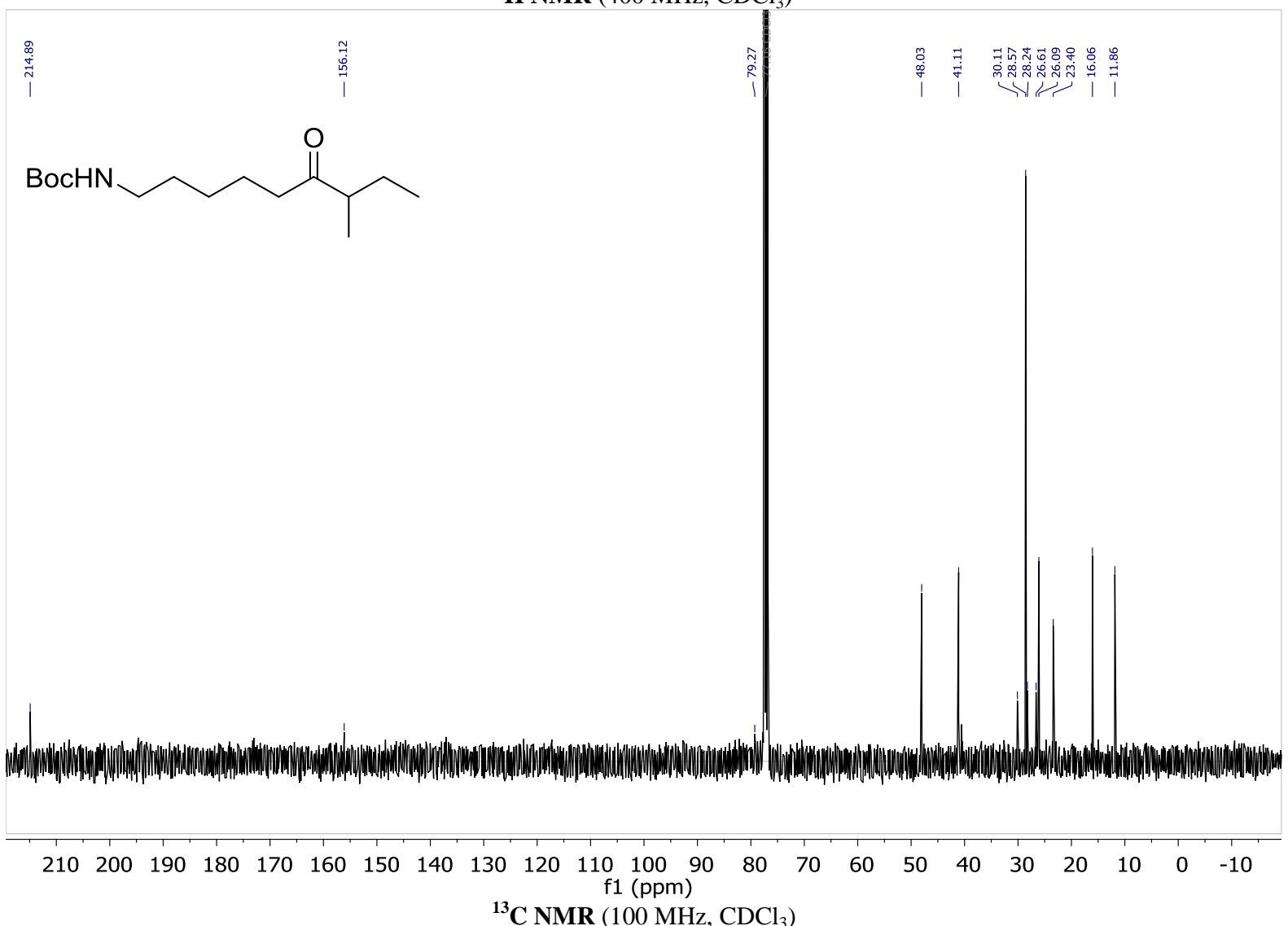




\subsubsection{Cyclic enimines}

TTOCT17-138

5-(prop-1-en-2-yl)-3,4-dihydro-2H-pyrrole hydrochloride 4a

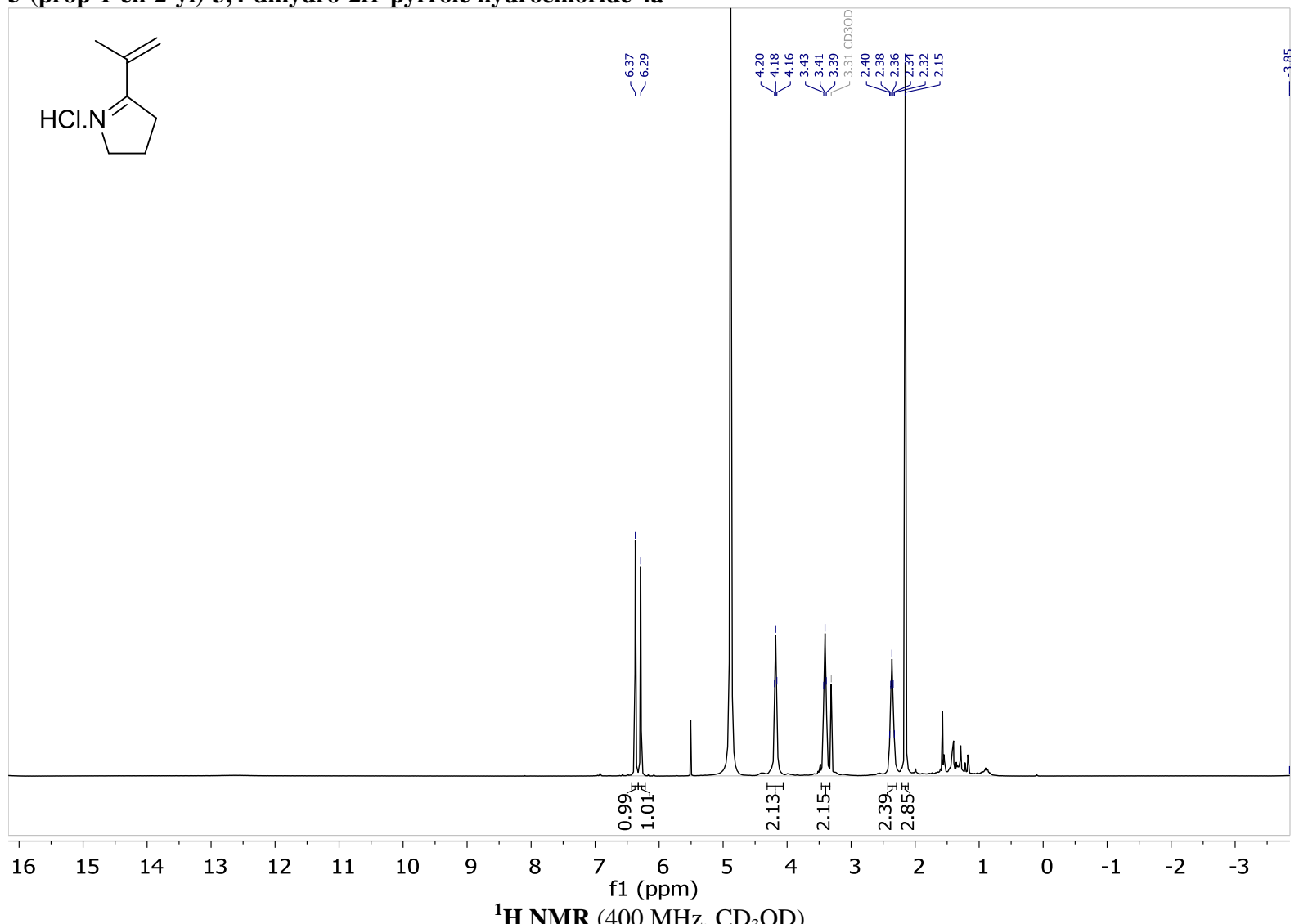

${ }^{1} \mathbf{H}$ NMR (400 MHz, CD 3 OD)

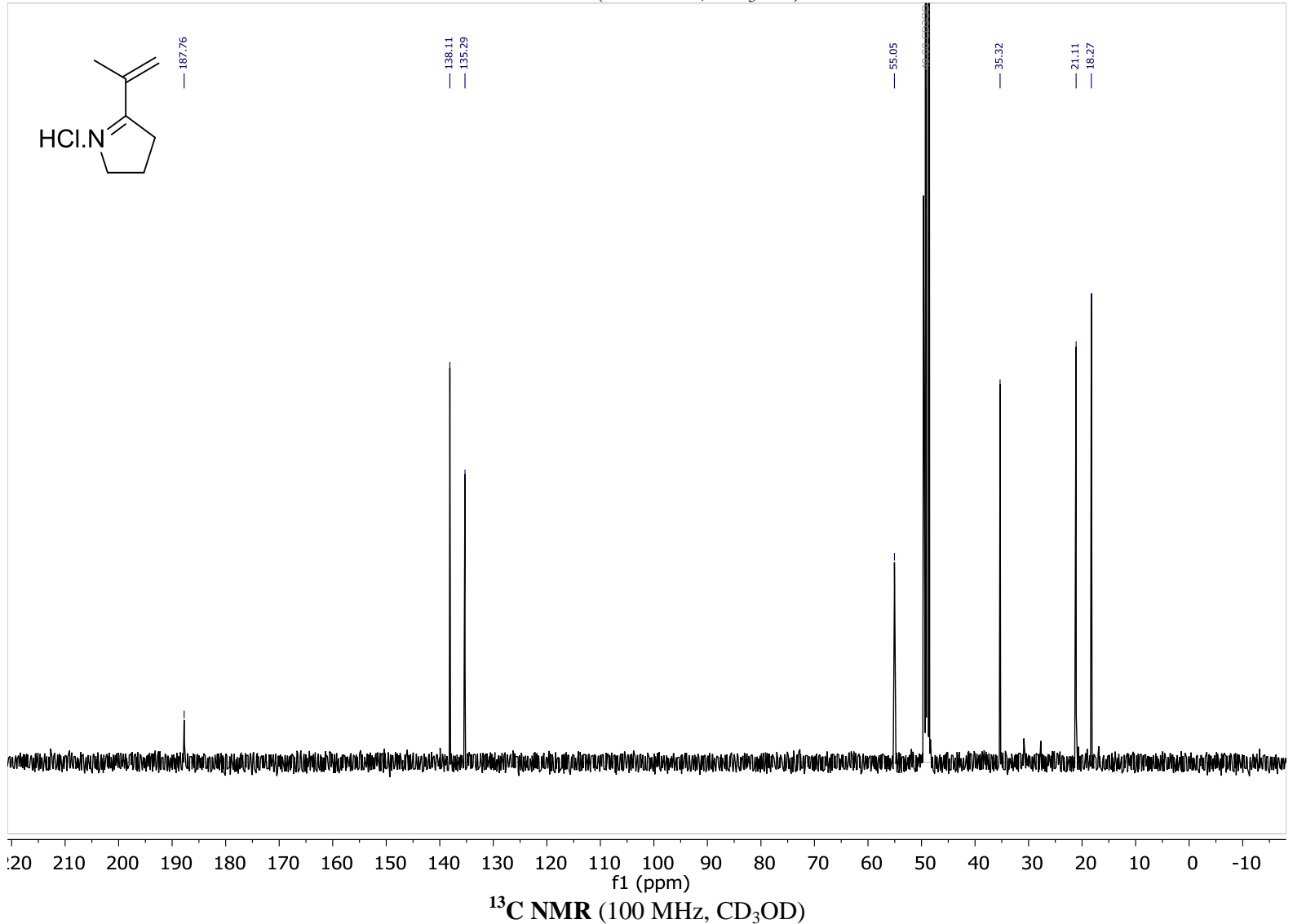


TTAUG18-65

5-(but-1-en-2-yl)-3,4-dihydro-2H-pyrrole trifluoroacetate 4b

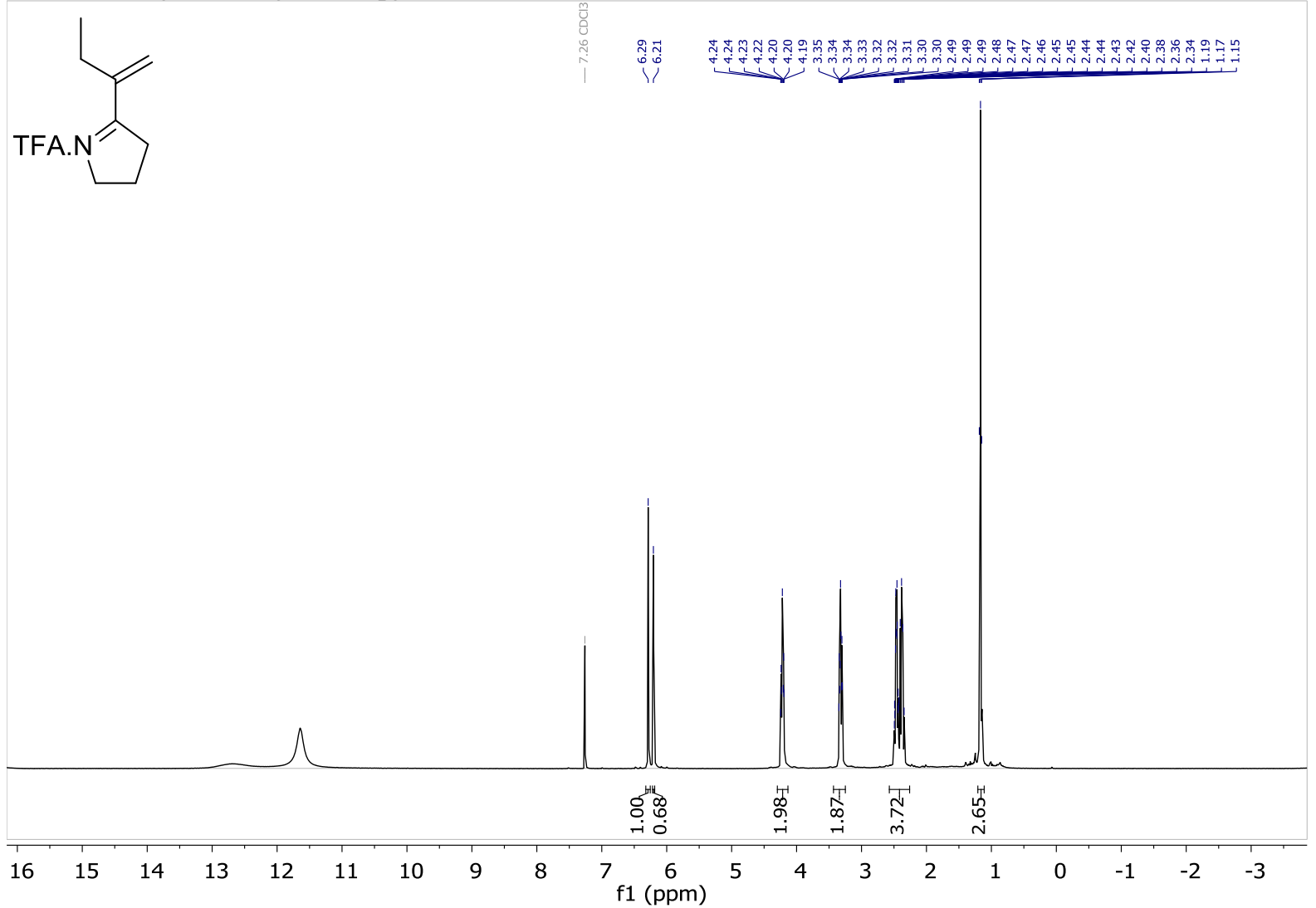

${ }^{1} \mathbf{H}$ NMR $\left(400 \mathrm{MHz}, \mathrm{CDCl}_{3}\right)$

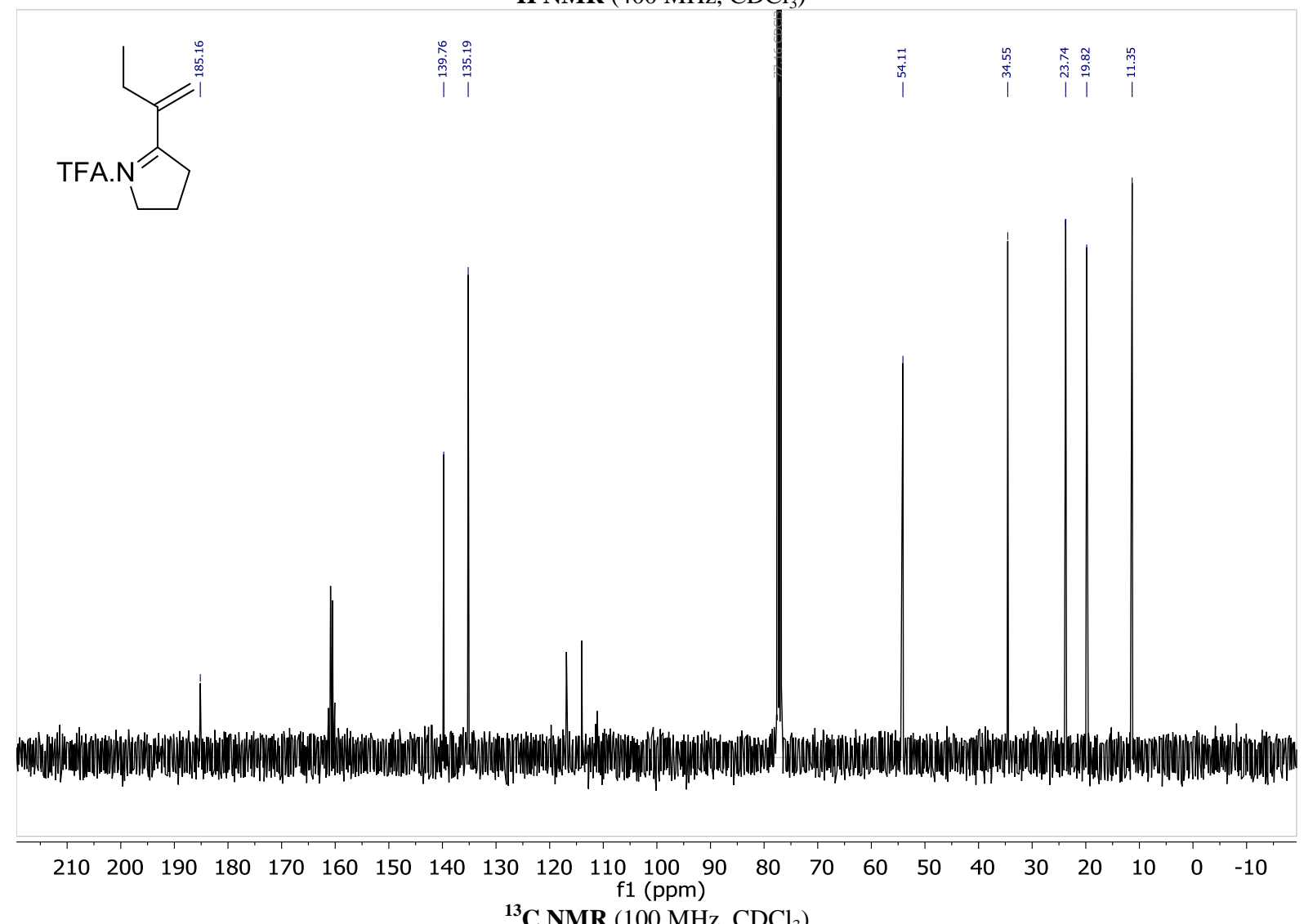

${ }^{13} \mathbf{C}$ NMR $\left(100 \mathrm{MHz}, \mathrm{CDCl}_{3}\right)$ 
TTAUG18-66

(E)-5-(but-2-en-2-yl)-3,4-dihydro-2H-pyrrole trifluoroacetate 4c

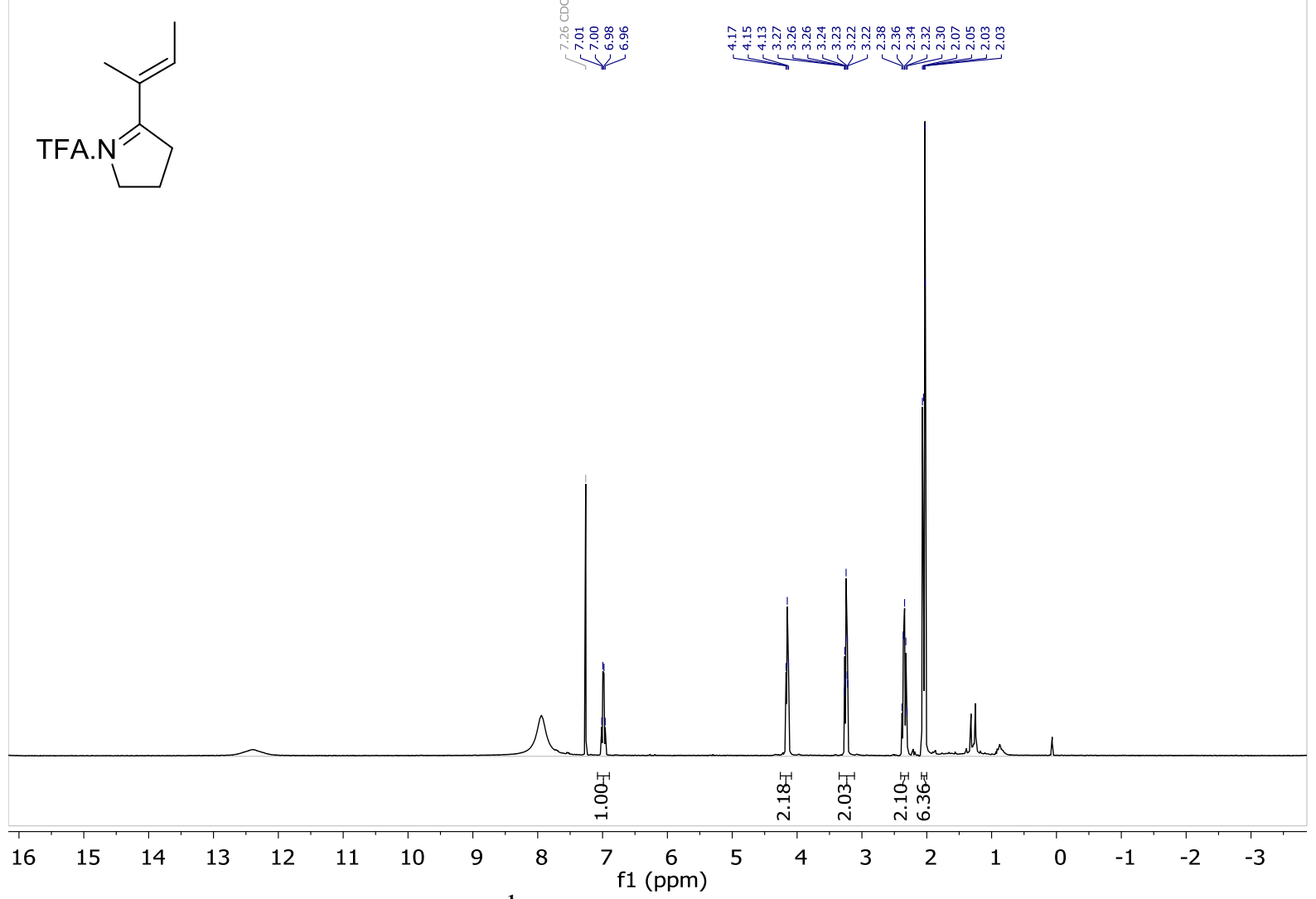

${ }^{1} \mathbf{H}$ NMR (400 MHz, $\mathrm{CDCl}_{3}$ )

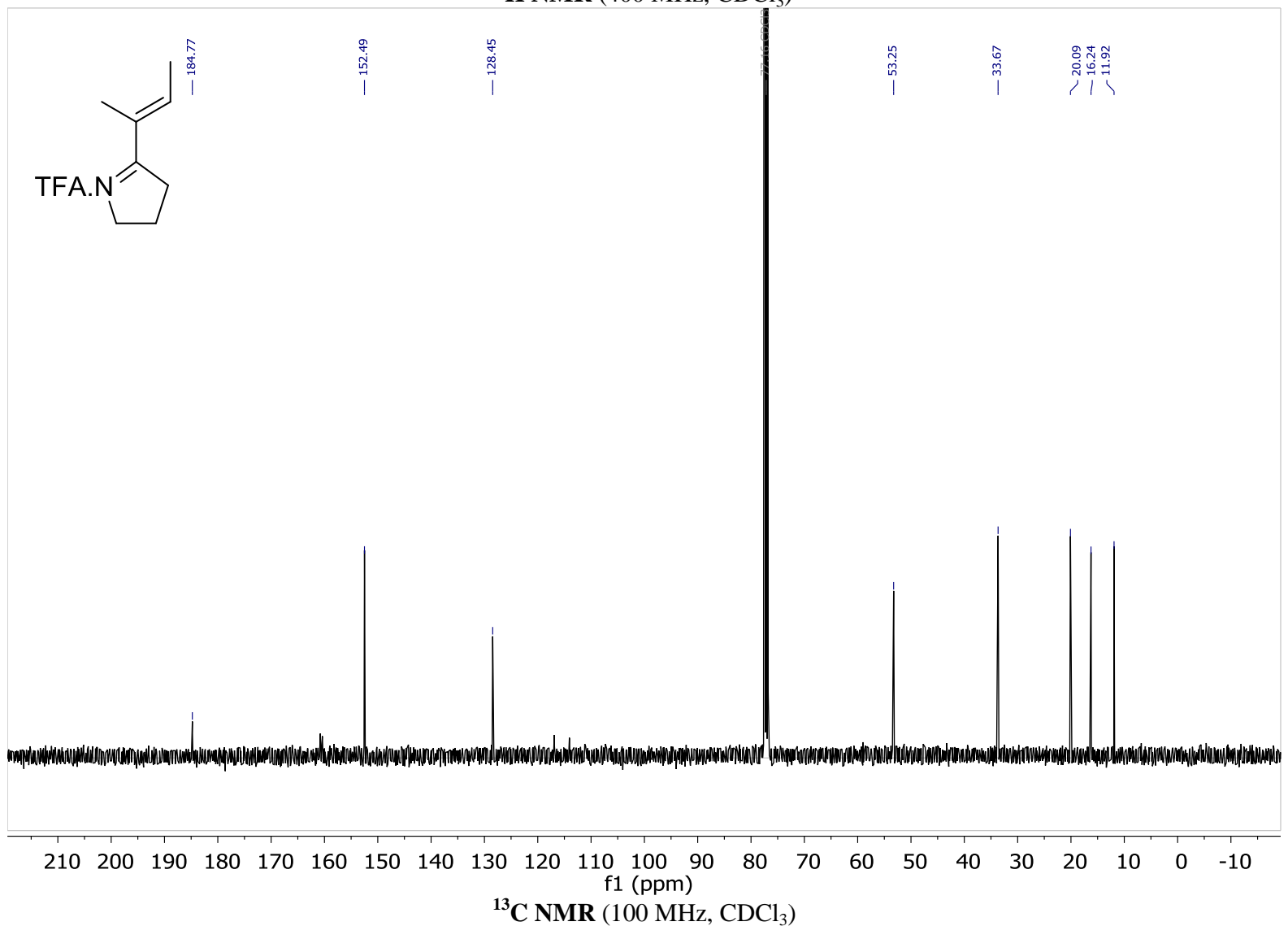

${ }^{13} \mathbf{C}$ NMR $\left(100 \mathrm{MHz}, \mathrm{CDCl}_{3}\right)$ 
TTOCT17-111

5-(2-methylprop-1-en-1-yl)-3,4-dihydro-2H-pyrrole hydrochloride 4d

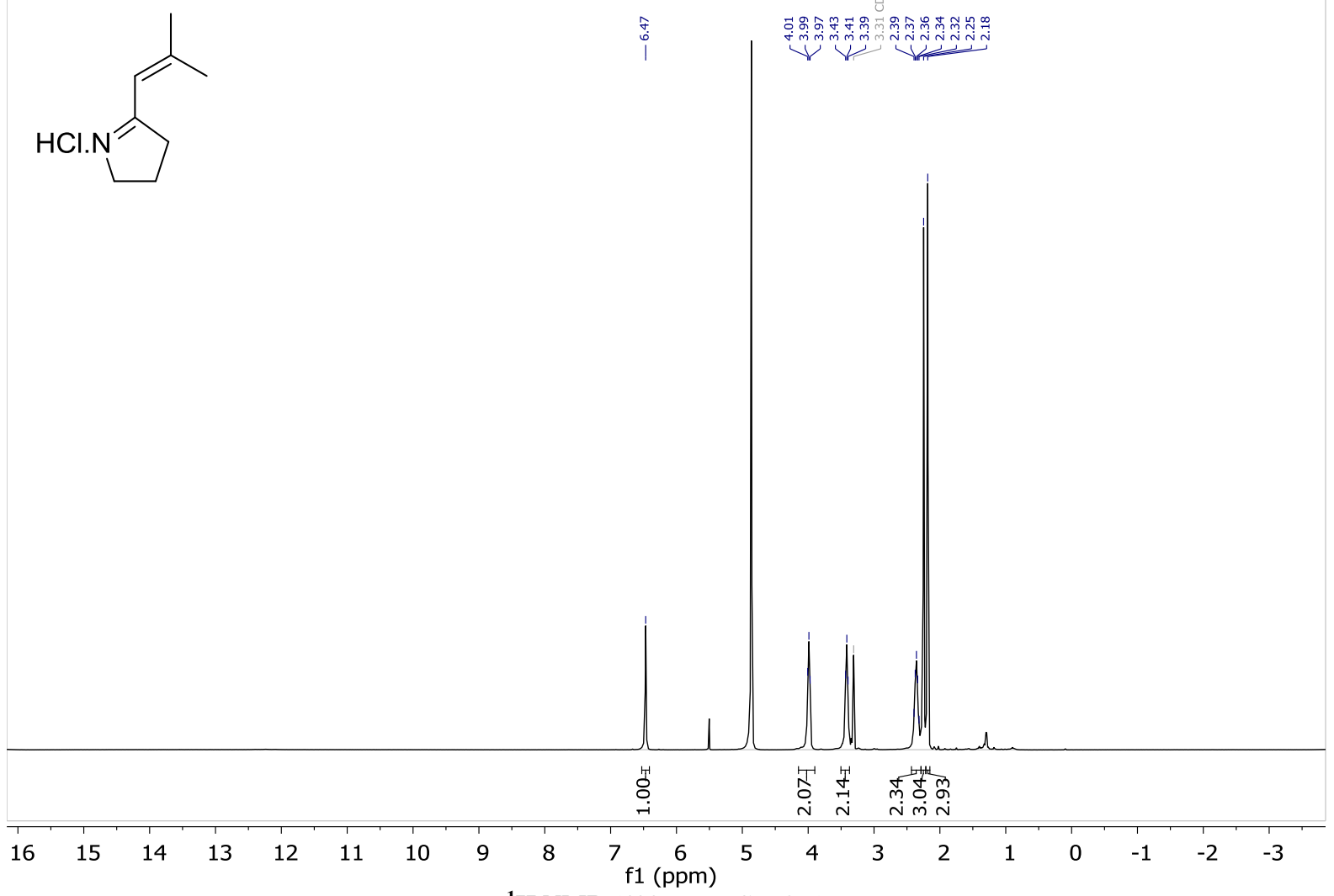

${ }^{1} \mathbf{H}$ NMR $\left(400 \mathrm{MHz}, \mathrm{CD}_{3} \mathrm{OD}\right)$

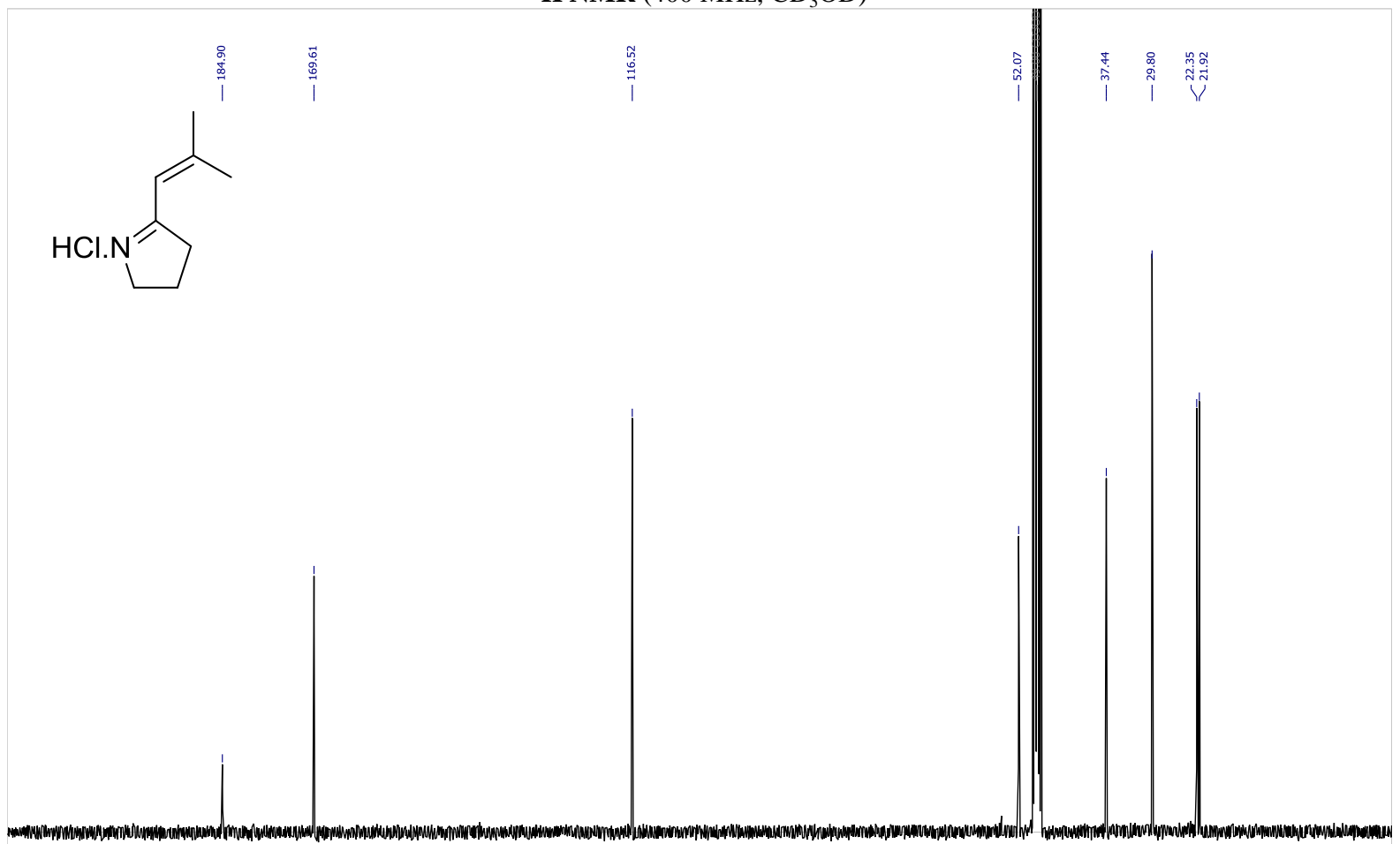

$\begin{array}{llllllllllllllllllllllll}20 & 210 & 200 & 190 & 180 & 170 & 160 & 150 & 140 & 130 & 120 & \begin{array}{c}110 \\ \mathrm{f} 1(\mathrm{ppm})\end{array} & 90 & 80 & 70 & 60 & 50 & 40 & 30 & 20 & 10 & 0 & -:\end{array}$

${ }^{13}$ C NMR (100 MHz, $\left.\mathrm{CD}_{3} \mathrm{OD}\right)$ 
TTAUG18-38

6-(prop-1-en-2-yl)-2,3,4,5-tetrahydropyridine hydrochloride 5a

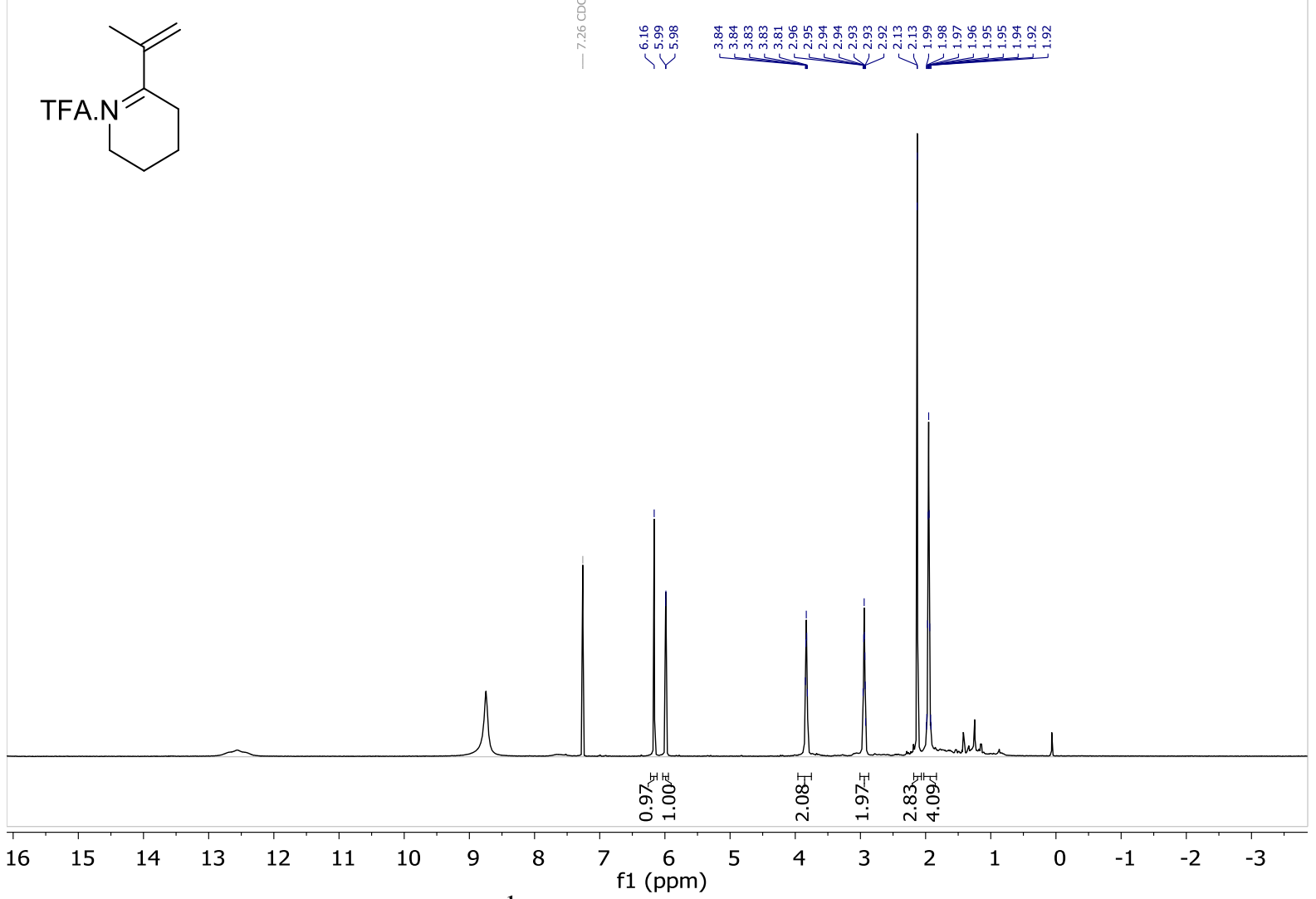

${ }^{1} \mathbf{H}$ NMR $\left(400 \mathrm{MHz}, \mathrm{CDCl}_{3}\right)$

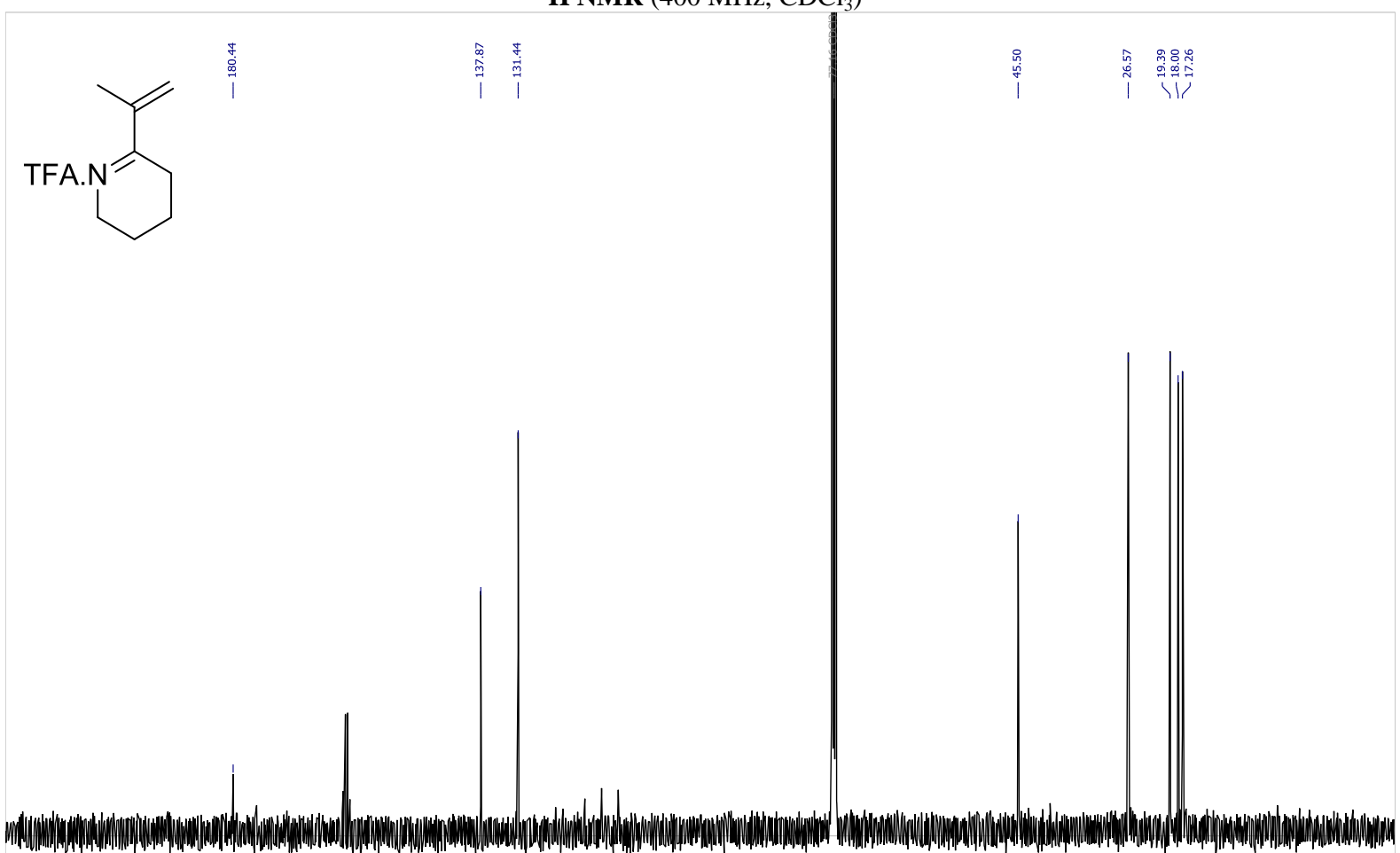

$\begin{array}{lllllllllllllllllllllll}210 & 200 & 190 & 180 & 170 & 160 & 150 & 140 & 130 & 120 & 110 \underset{\mathrm{f} 1(\mathrm{ppm})}{100} & 90 & 80 & 70 & 60 & 50 & 40 & 30 & 20 & 10 & 0 & -10\end{array}$

${ }^{13}$ C NMR $\left(100 \mathrm{MHz}, \mathrm{CDCl}_{3}\right)$ 
TTAUG18-29

6-(but-1-en-2-yl)-2,3,4,5-tetrahydropyridine trifluoroacetate 5b

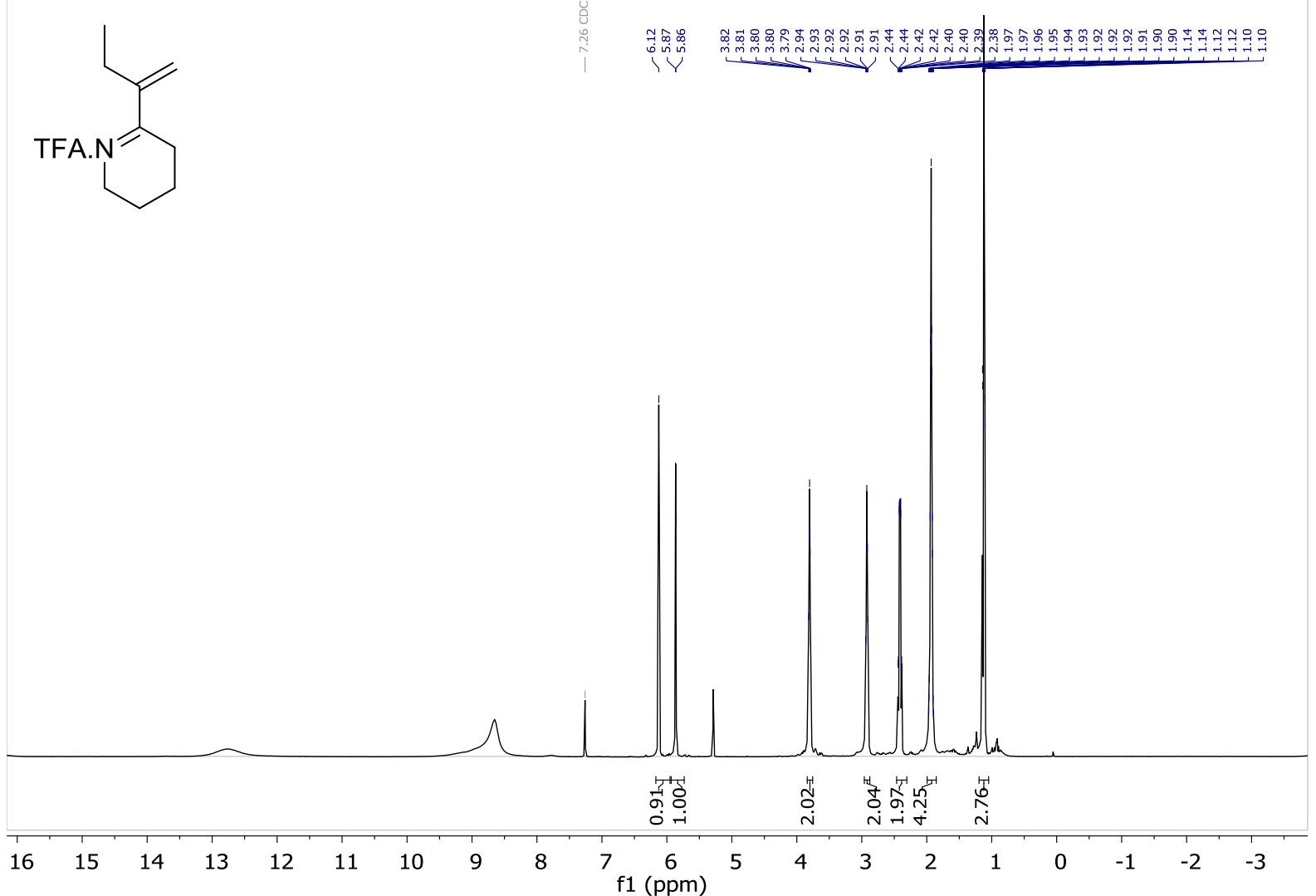

${ }^{1} \mathbf{H}$ NMR $\left(400 \mathrm{MHz}, \mathrm{CDCl}_{3}\right)$

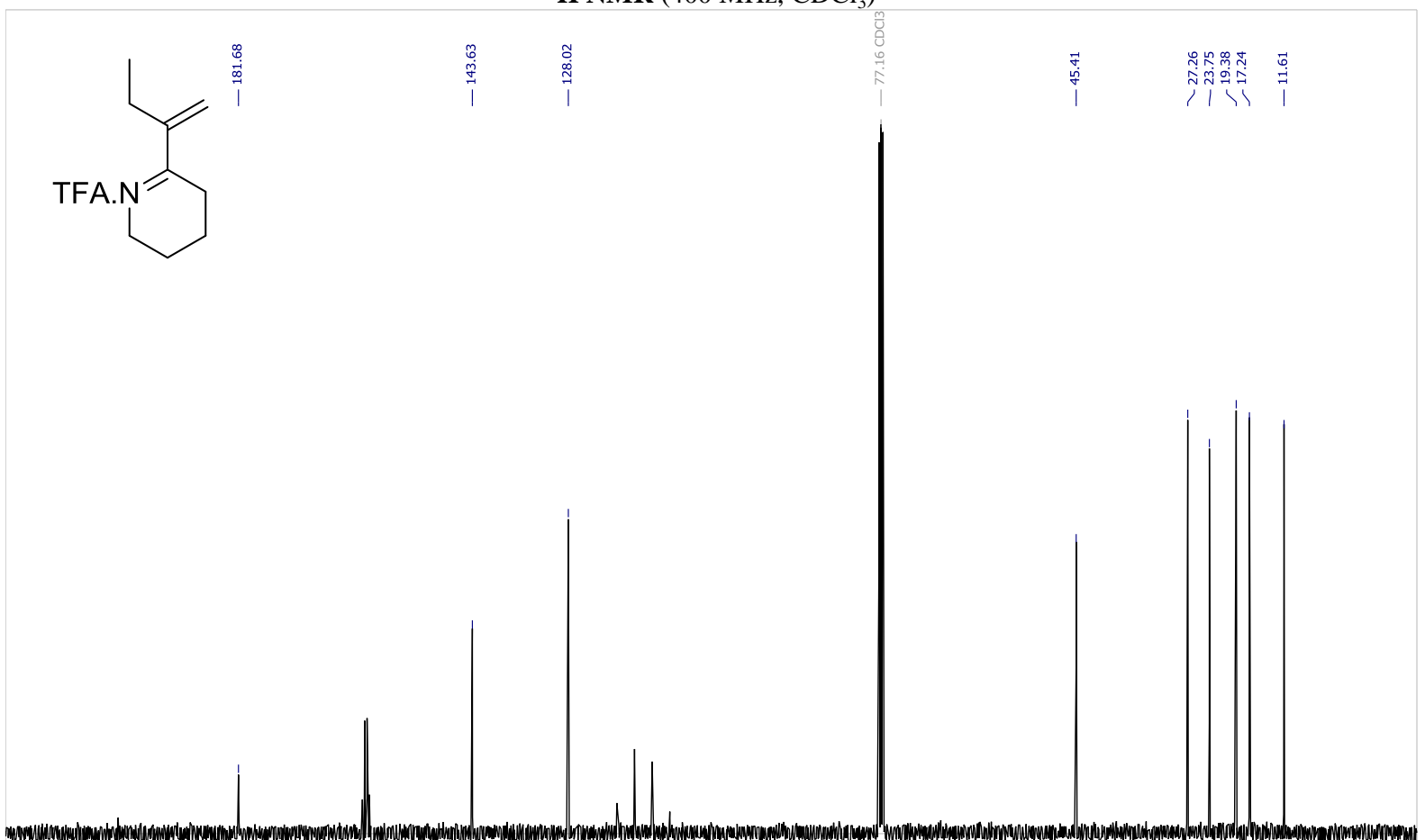

$\begin{array}{lllllllllllllllllllllll}210 & 200 & 190 & 180 & 170 & 160 & 150 & 140 & 130 & 120 & 110 & 100 & 90 & 80 & 70 & 60 & 50 & 40 & 30 & 20 & 10 & 0 & -\end{array}$ ${ }^{13}$ C NMR $\left(100 \mathrm{MHz}, \mathrm{CDCl}_{3}\right)$ 
TTAUG18-37

(E)-6-(but-2-en-2-yl)-2,3,4,5-tetrahydropyridine 5c

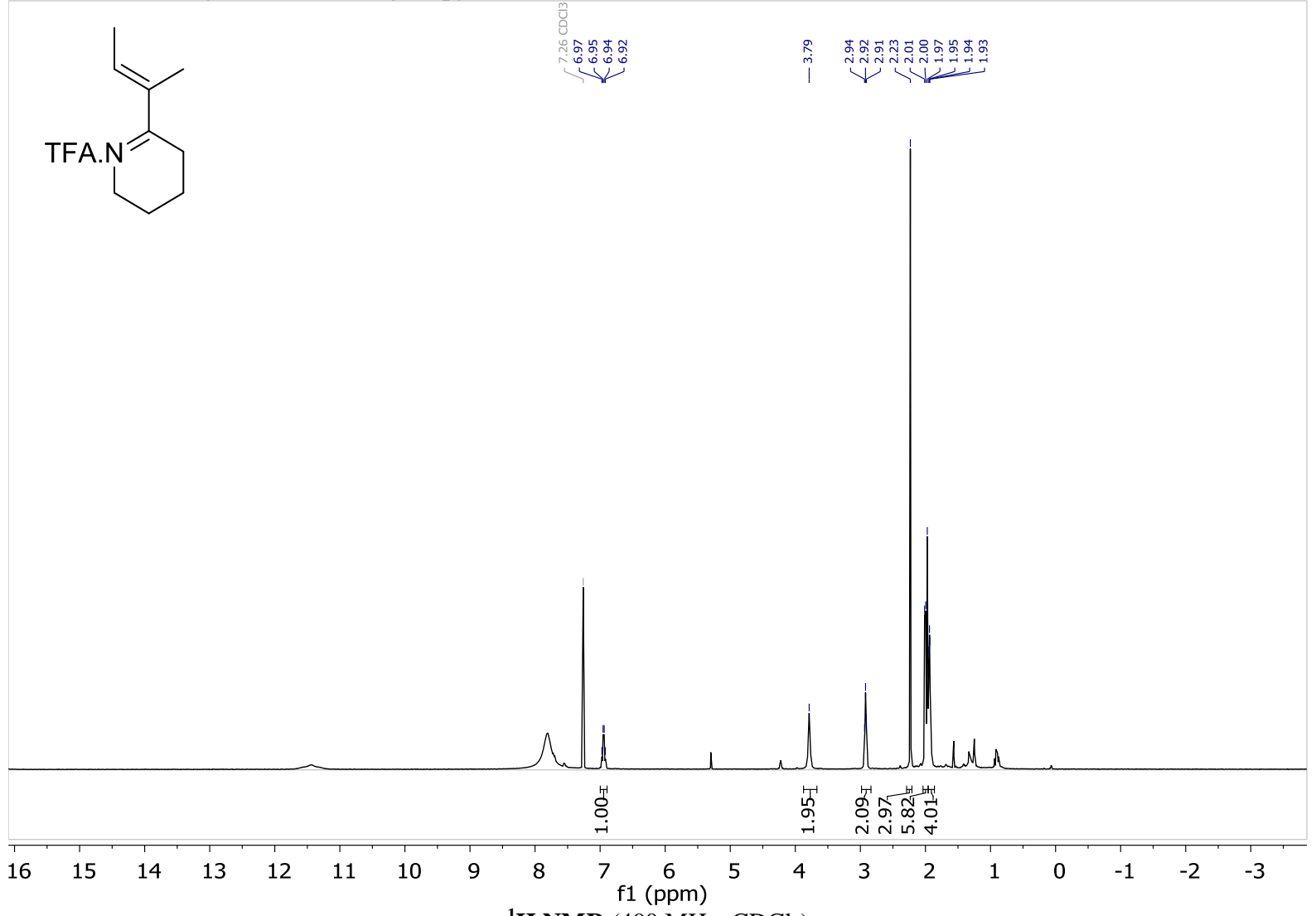

${ }^{1} \mathbf{H}$ NMR $\left(400 \mathrm{MHz}, \mathrm{CDCl}_{3}\right)$

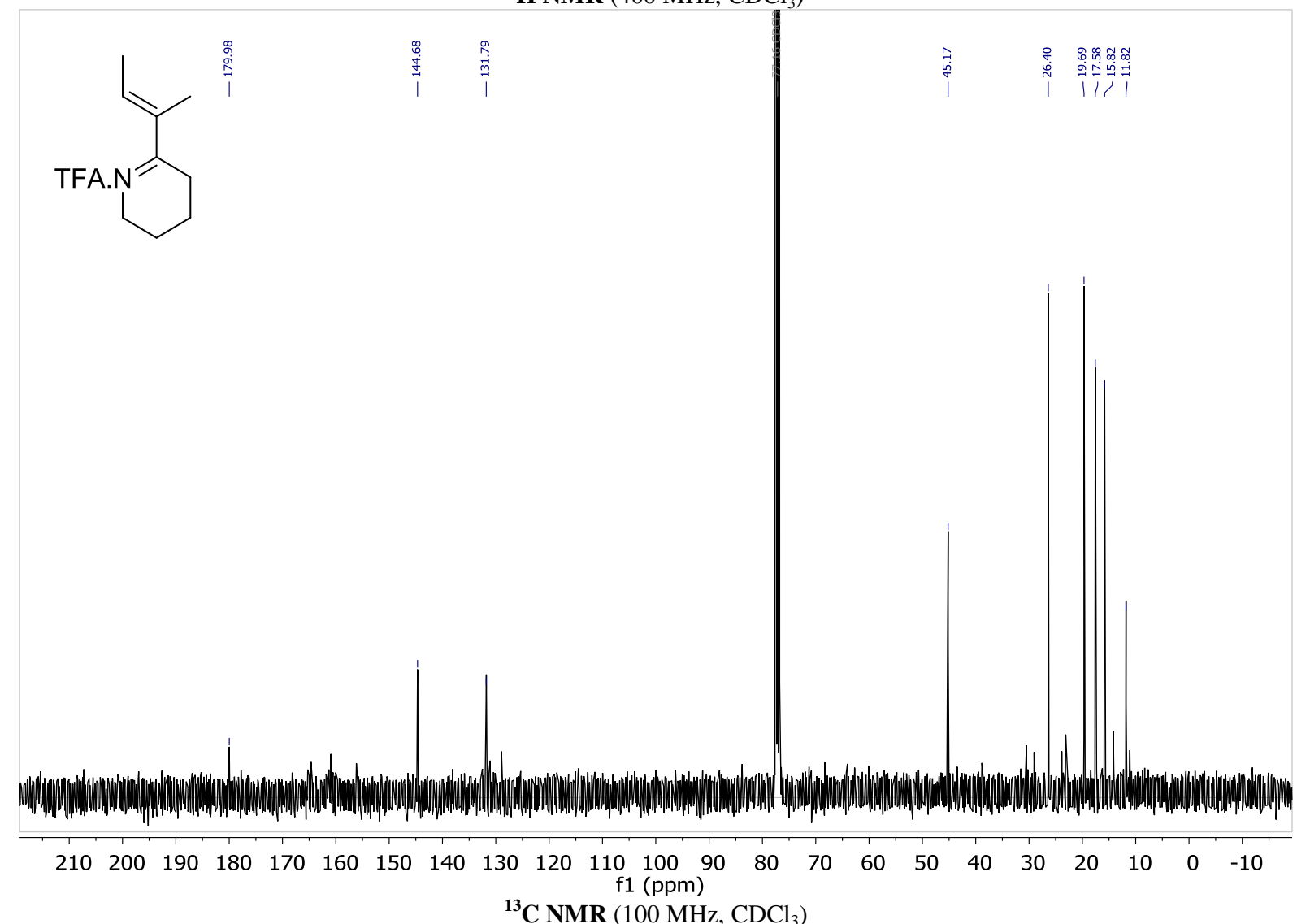


TTOCT17-114

6-(2-methylprop-1-en-1-yl)-2,3,4,5-tetrahydropyridine hydrochloride 5d

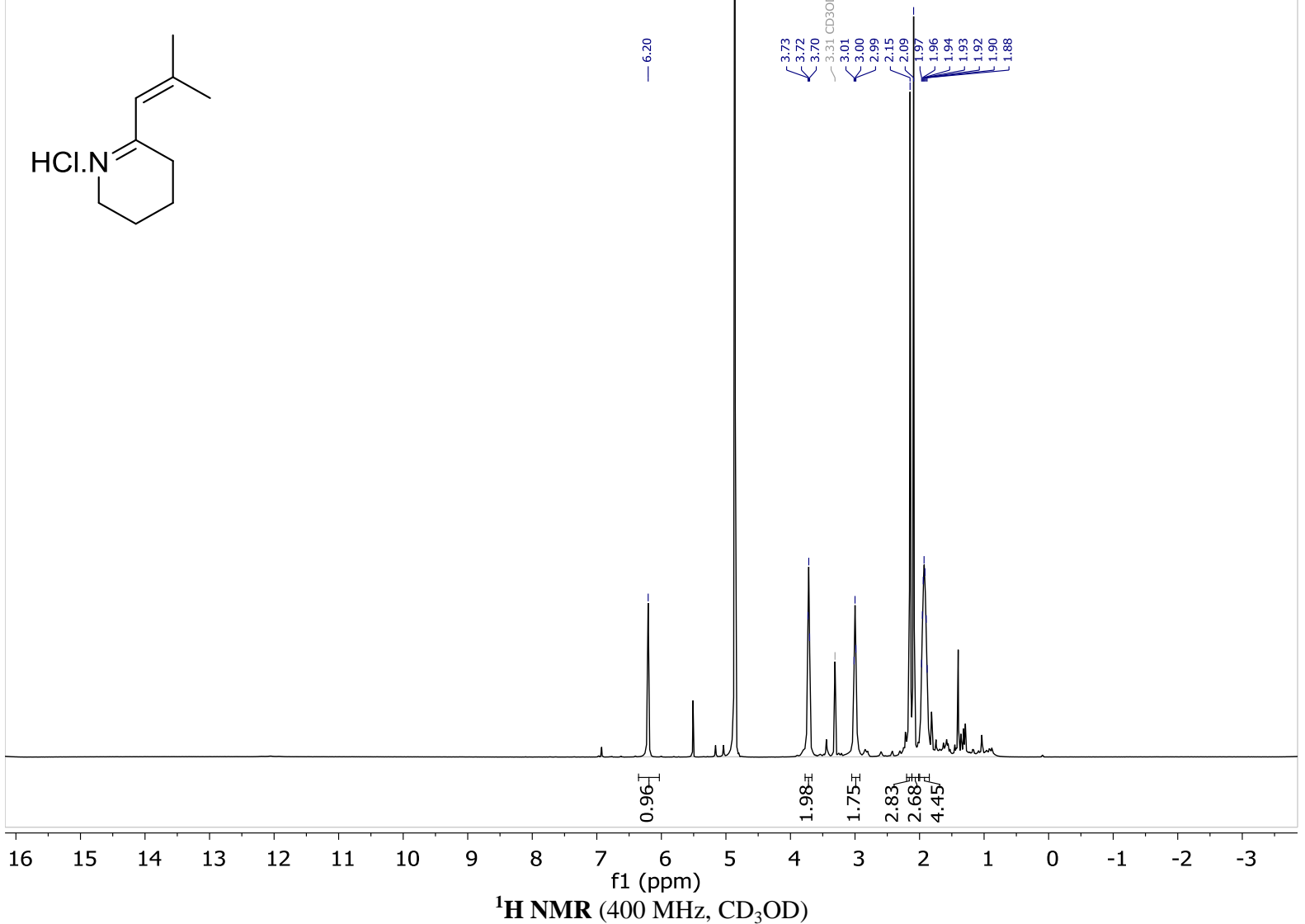

${ }^{1} \mathbf{H}$ NMR $\left(400 \mathrm{MHz}, \mathrm{CD}_{3} \mathrm{OD}\right)$

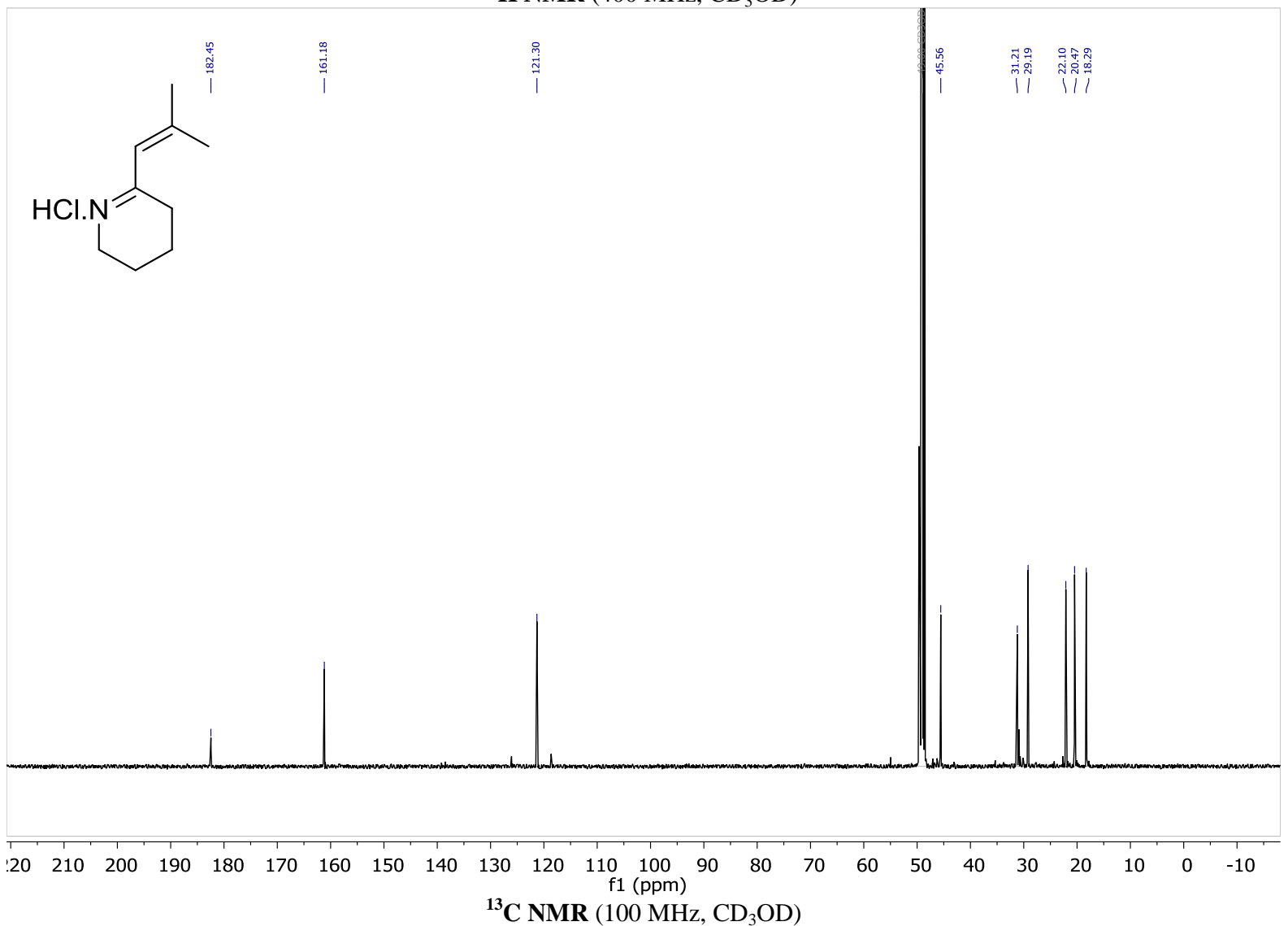


TTAUG18-36

8-amino-2-methyloct-1-en-3-one trifluoroacetate 6a

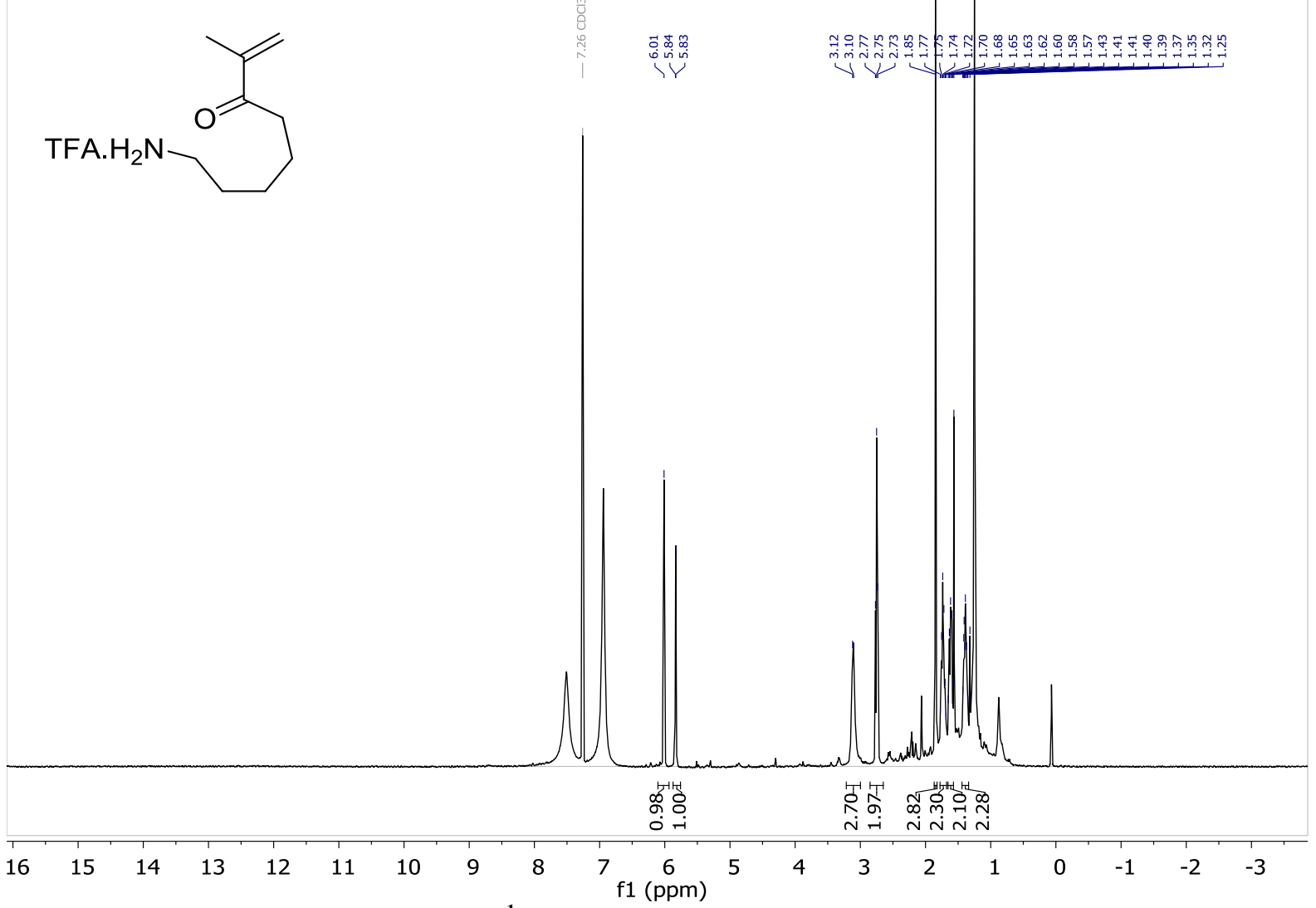

${ }^{1} \mathbf{H}$ NMR $\left(400 \mathrm{MHz}, \mathrm{CDCl}_{3}\right)$

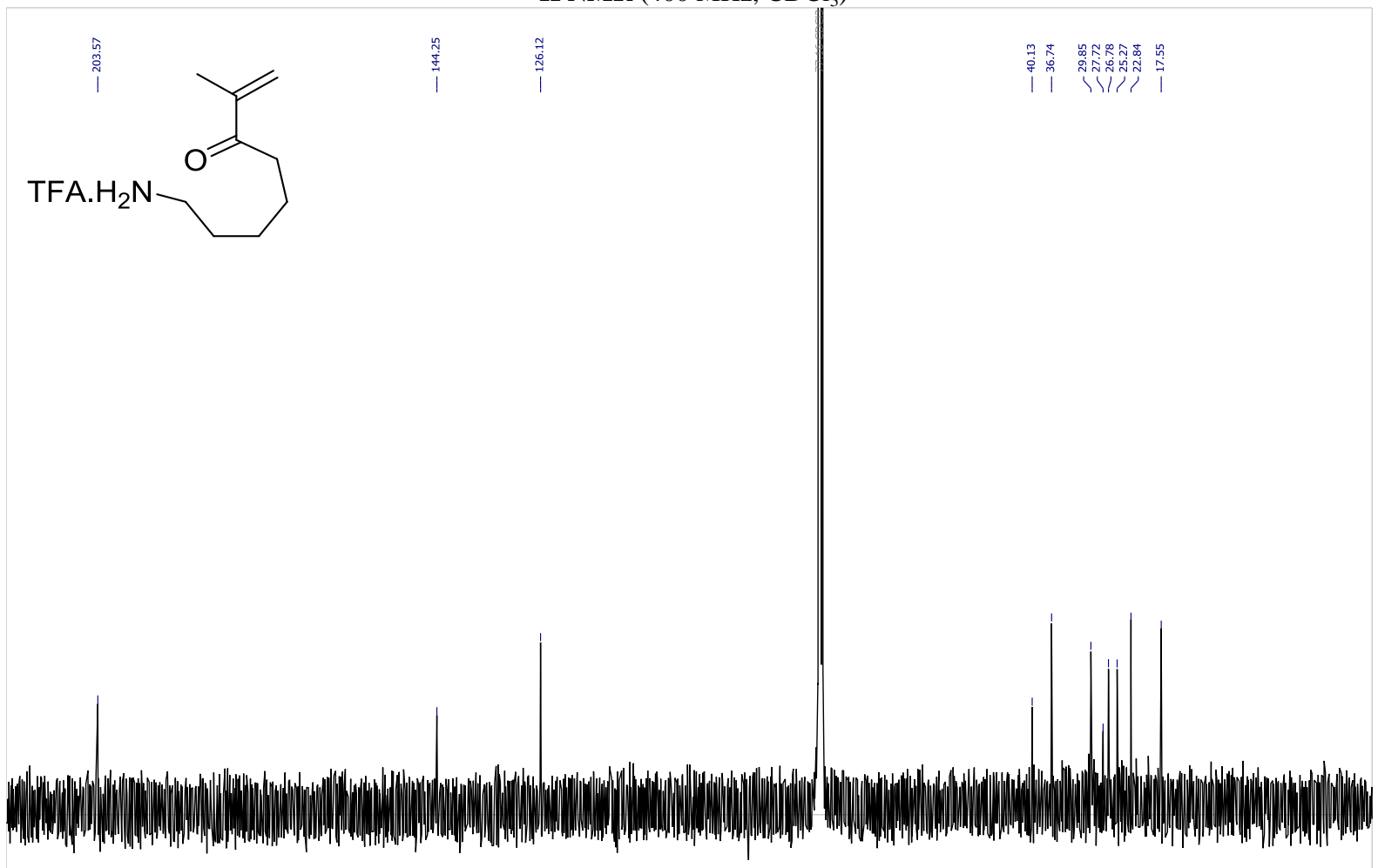

$\begin{array}{lllllllllllllllllllllll}210 & 200 & 190 & 180 & 170 & 160 & 150 & 140 & 130 & 120 & 110 & 100 & 100 & 80 & 70 & 60 & 50 & 40 & 30 & 20 & 10 & 0 & -10\end{array}$ ${ }^{13}$ C NMR $\left(100 \mathrm{MHz}, \mathrm{CDCl}_{3}\right)$ 
TTAUG18-113

9-amino-3-methylenenonan-4-one trifluoroacetate $6 \mathrm{~b}$

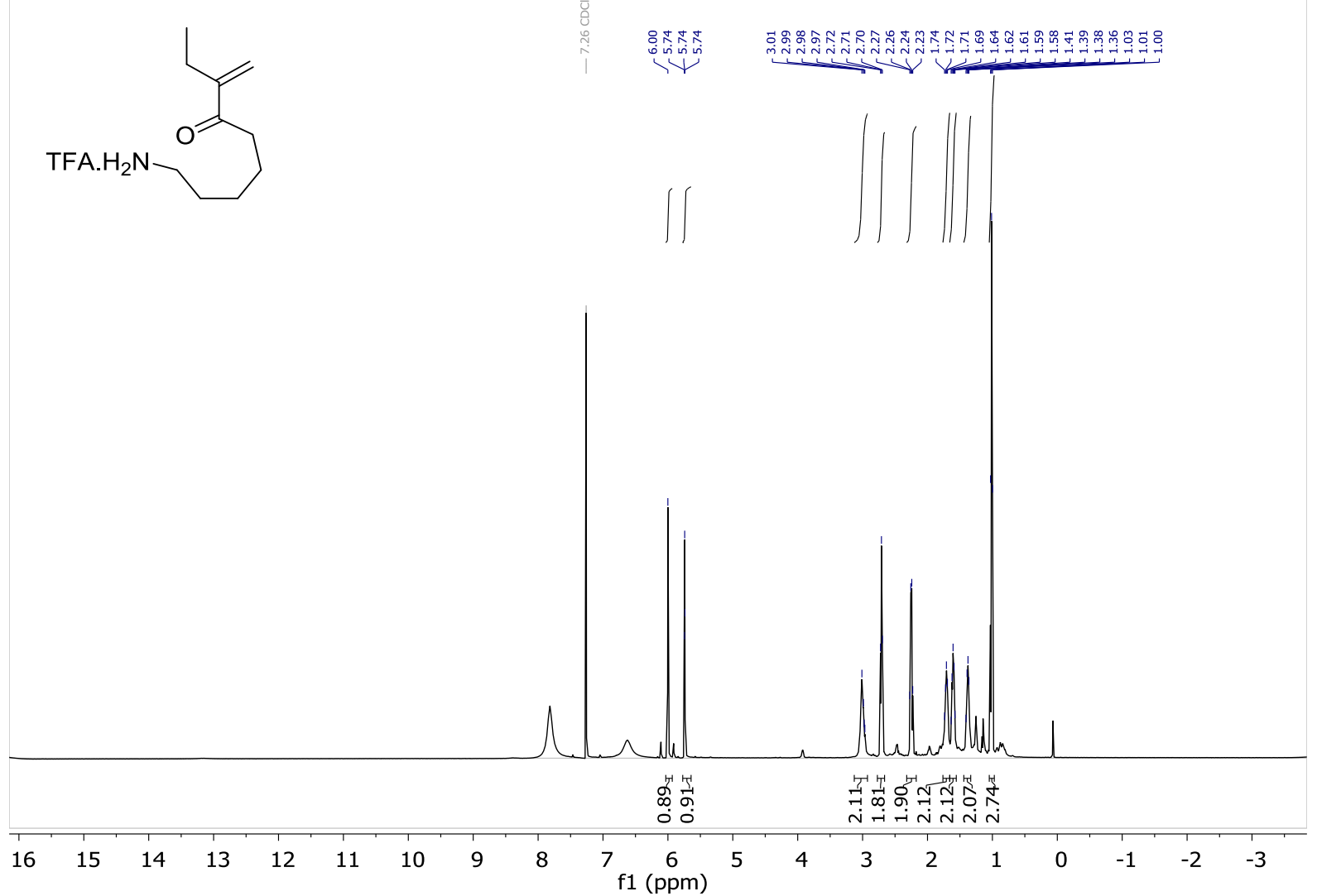

${ }^{1} \mathbf{H}$ NMR (400 MHz, $\left.\mathrm{CDCl}_{3}\right)$

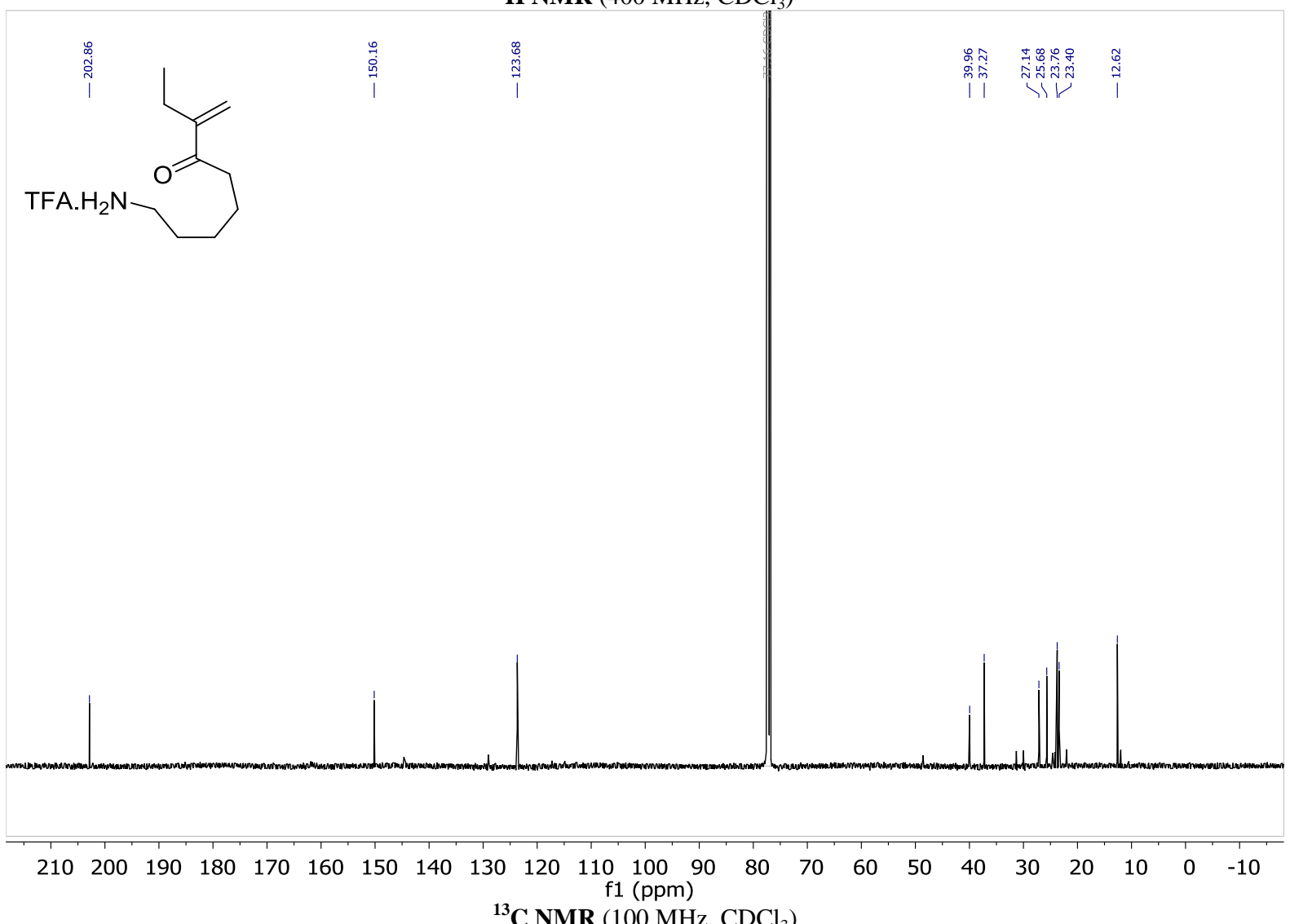

${ }^{13} \mathbf{C}$ NMR $\left(100 \mathrm{MHz}, \mathrm{CDCl}_{3}\right)$ 
TTAUG18-114

(E)-8-amino-3-methyloct-2-en-4-one trifluoroacetate 6c

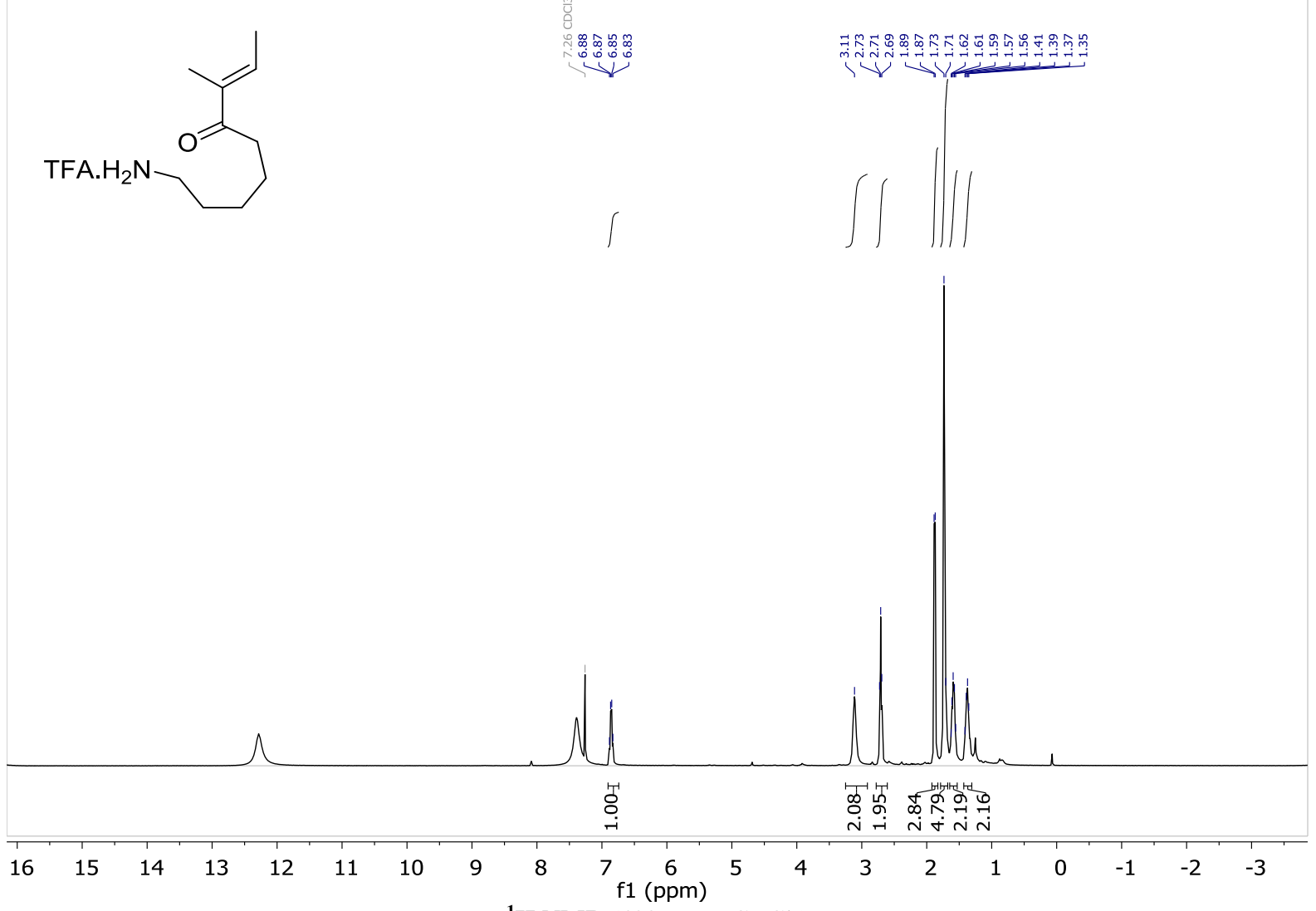

${ }^{1} \mathbf{H}$ NMR $\left(400 \mathrm{MHz}, \mathrm{CDCl}_{3}\right)$

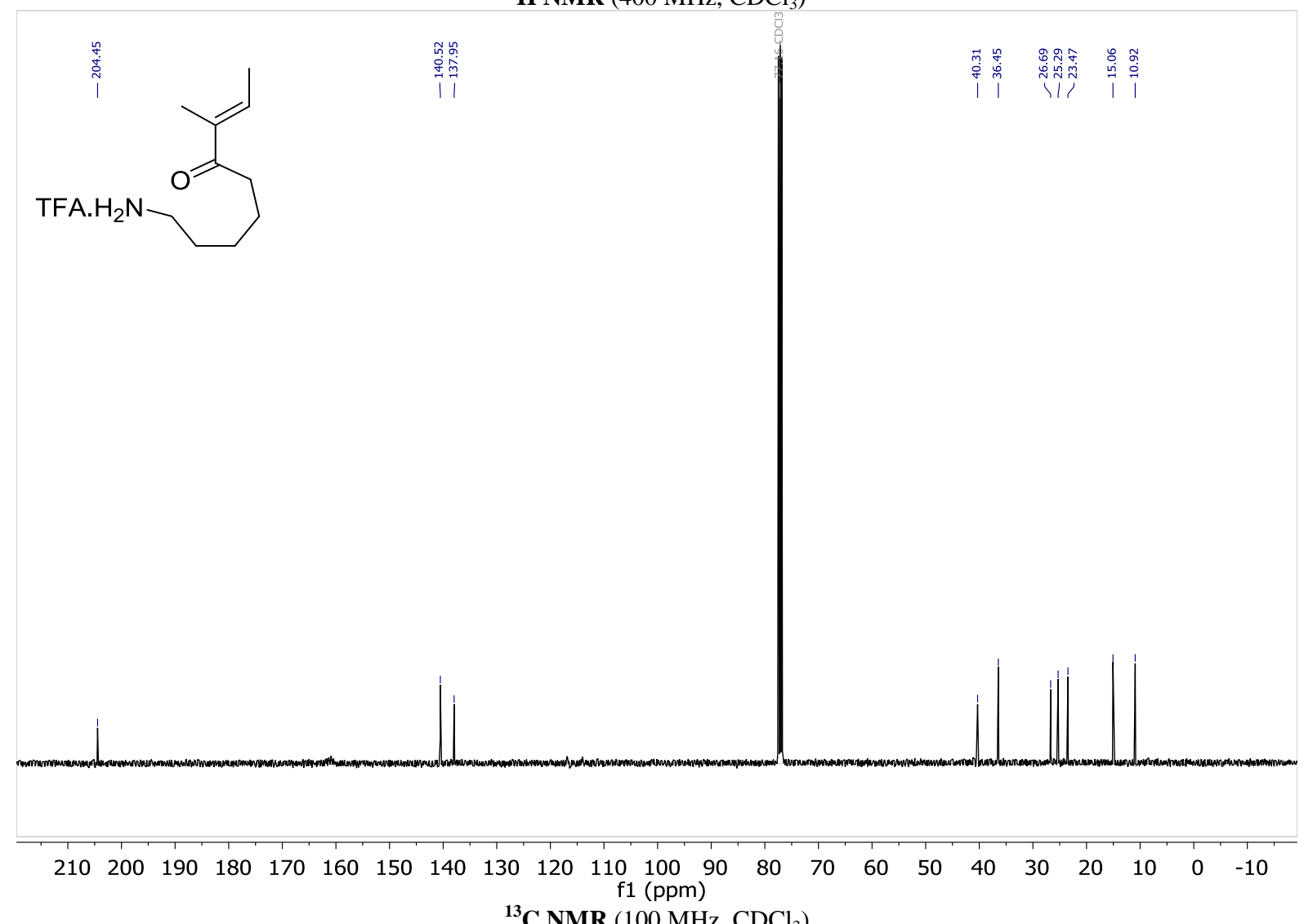


TTAUG18-96

9-amino-2-methylnon-2-en-4-one trifluoroacetate 6d

${ }^{1} \mathbf{H}$ NMR $\left(400 \mathrm{MHz}, \mathrm{CDCl}_{3}\right)$

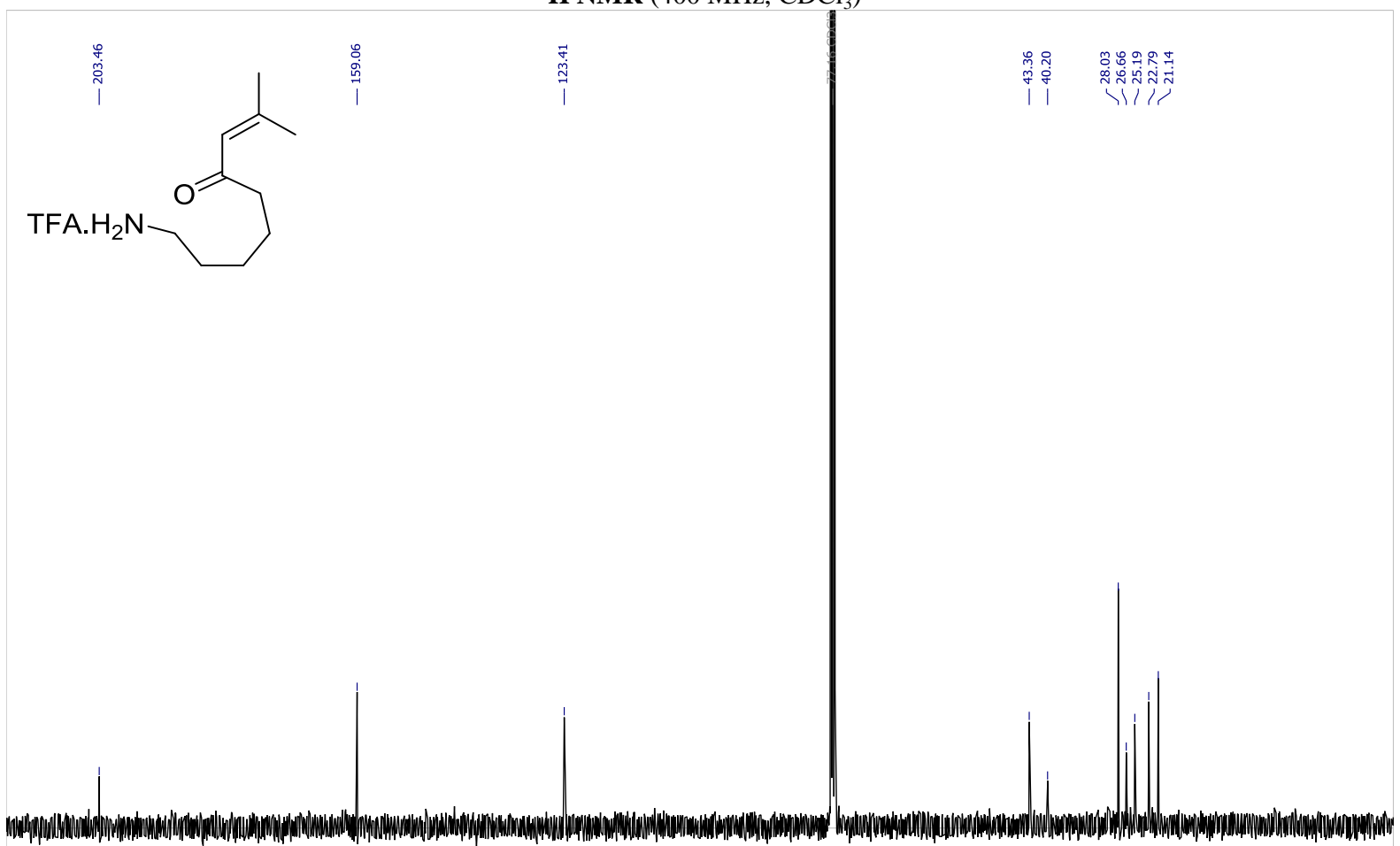

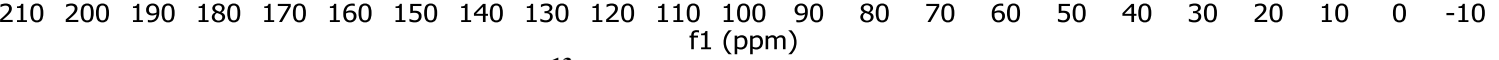
${ }^{13} \mathbf{C} \mathbf{N M R}\left(100 \mathrm{MHz}, \mathrm{CDCl}_{3}\right)$ 


\subsubsection{Cyclic imines}

TTAUG18-1

5-isopropyl-3,4-dihydro-2H-pyrrole hydrochloride 13a

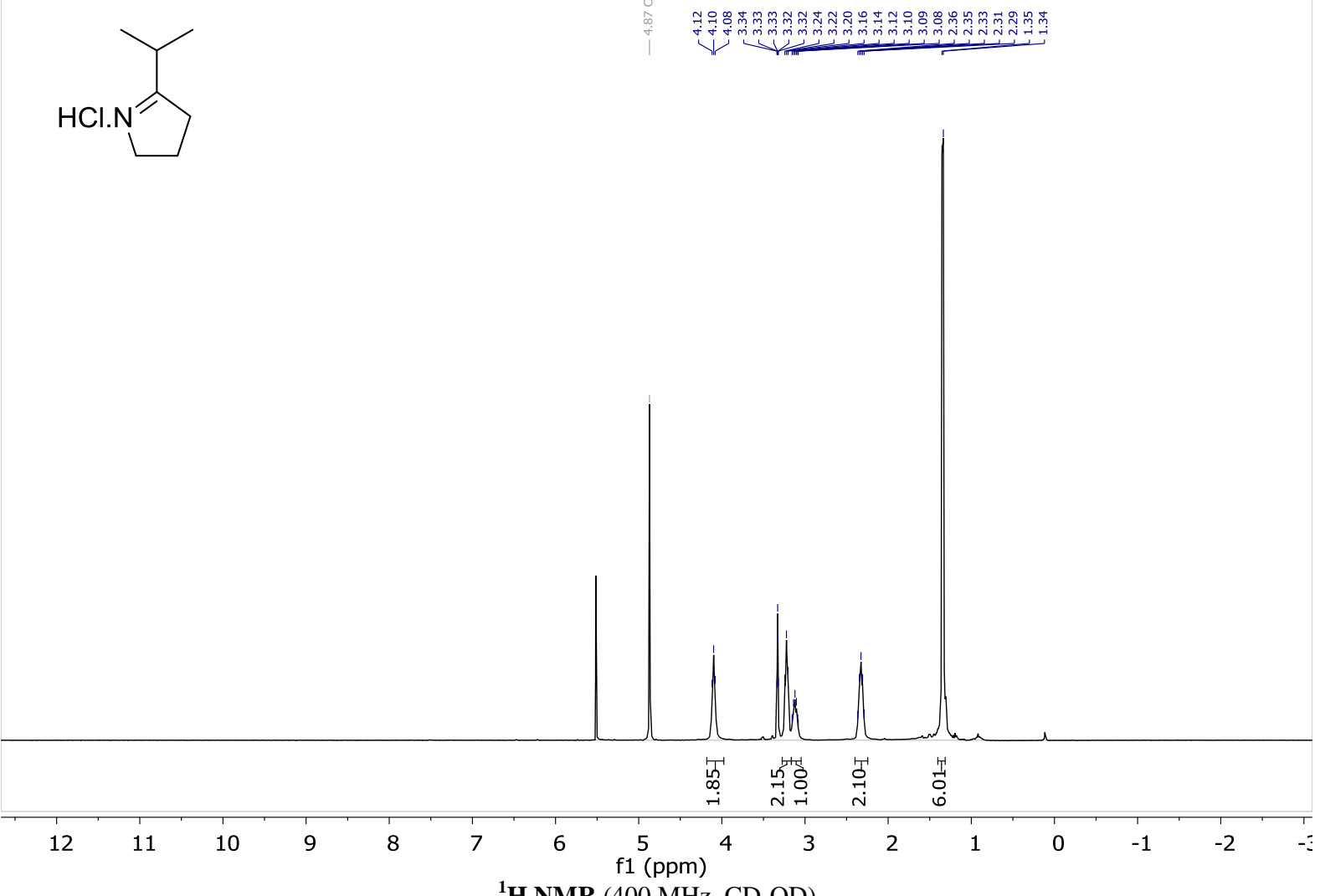

${ }^{1}$ H NMR (400 MHz, CD 3 OD)

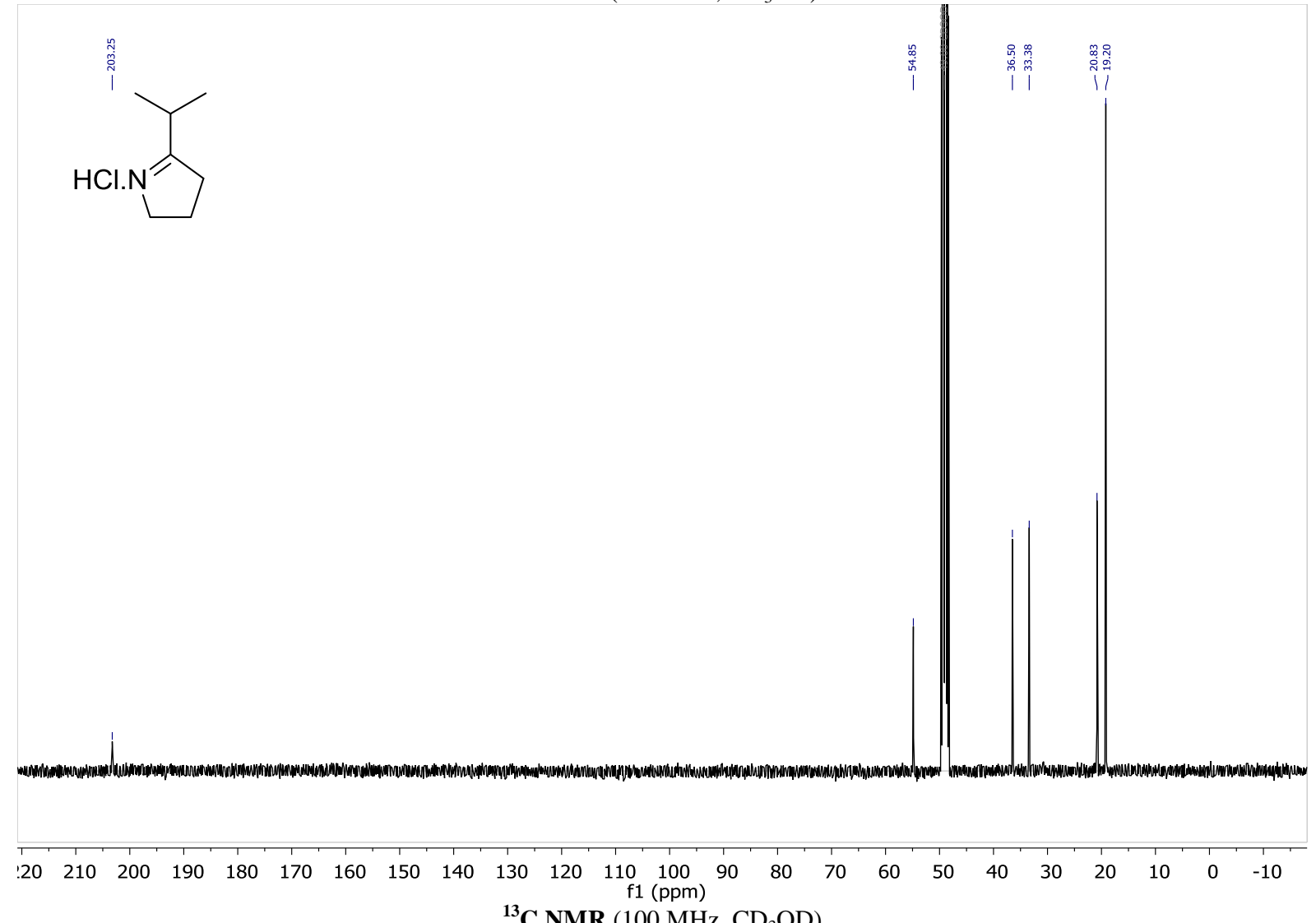

${ }^{13} \mathbf{C}$ NMR (100 MHz, $\left.\mathrm{CD}_{3} \mathrm{OD}\right)$ 
TTAUG18-68

5-(sec-butyl)-3,4-dihydro-2H-pyrrole hydrochloride $13 \mathrm{~b}$

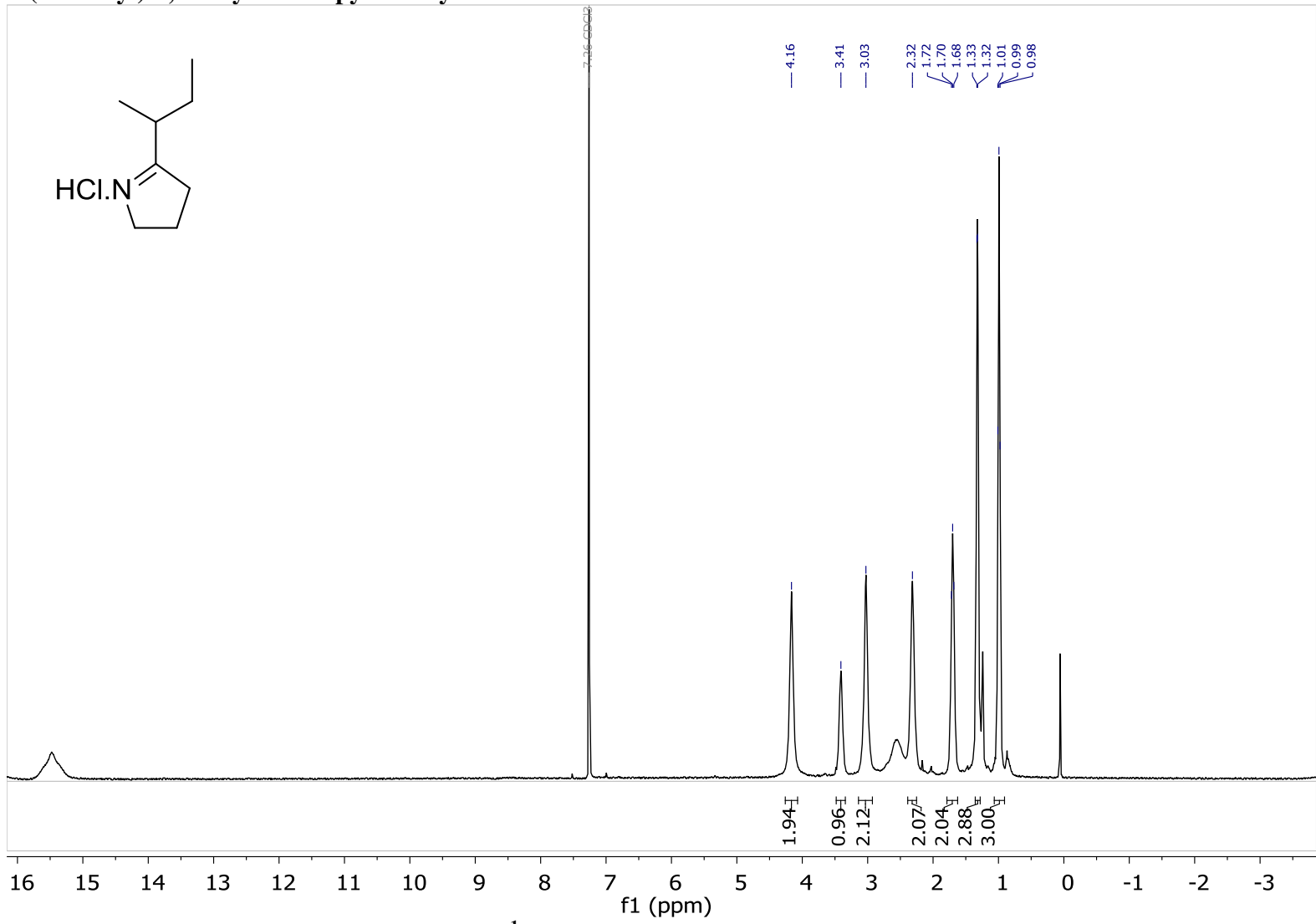

${ }^{1} \mathbf{H}$ NMR $\left(400 \mathrm{MHz}, \mathrm{CDCl}_{3}\right)$

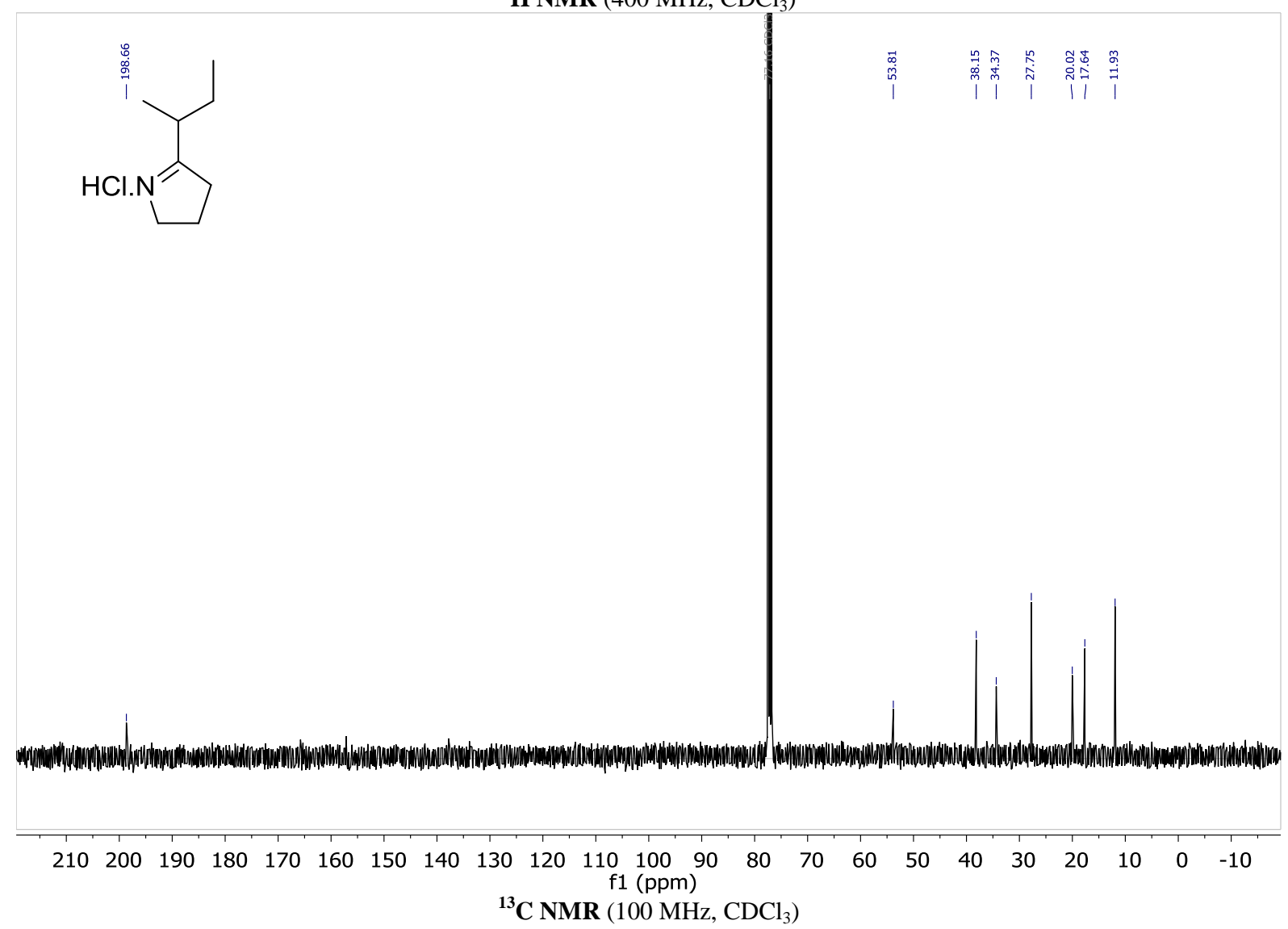


TTAUG18-7

5-isobutyl-3,4-dihydro-2H-pyrrole hydrochloride 13d

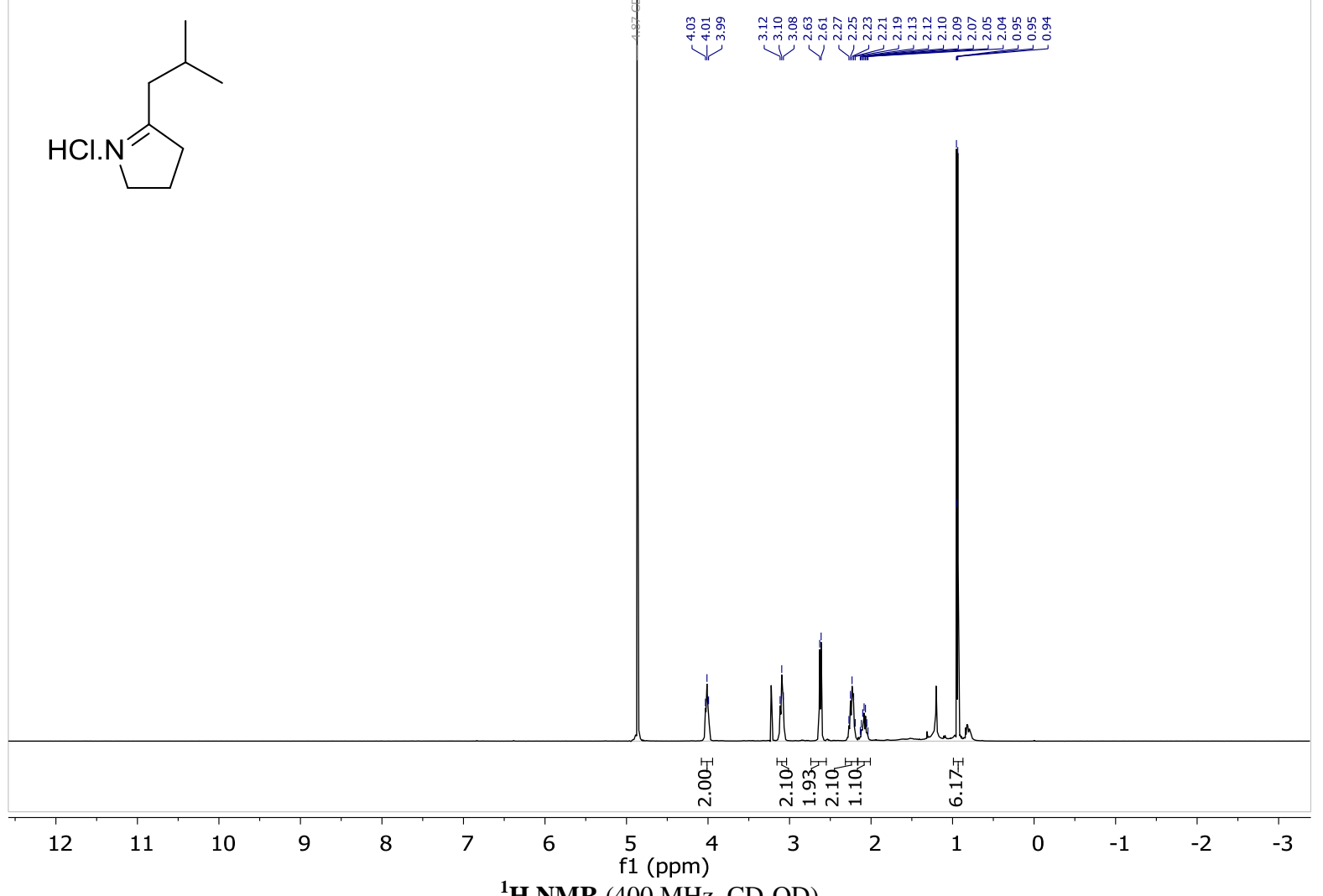

${ }^{1}$ H NMR (400 MHz, $\left.\mathrm{CD}_{3} \mathrm{OD}\right)$

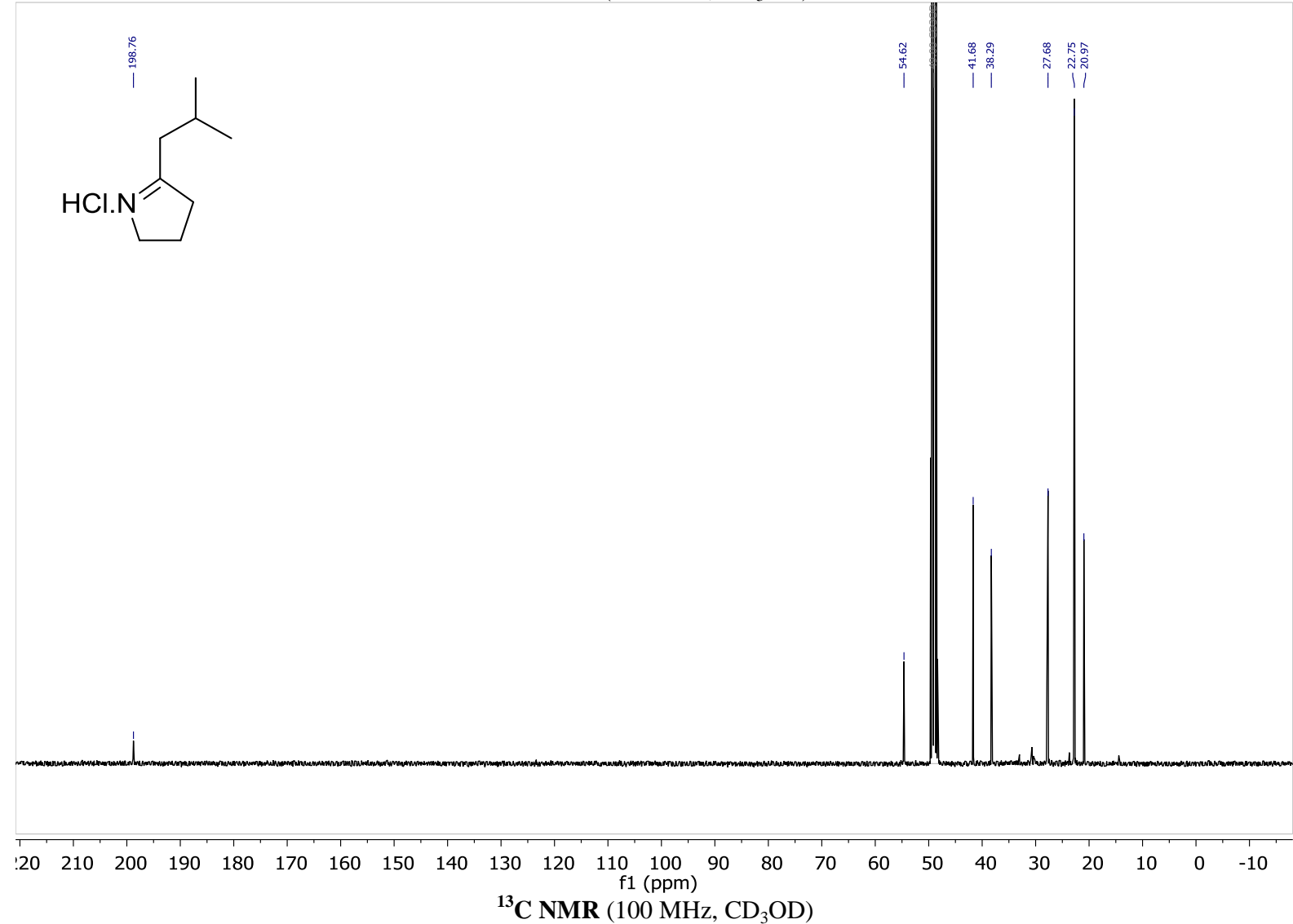


TTOCT17-96

6-isopropyl-2,3,4,5-tetrahydropyridine hydrochloride 14a

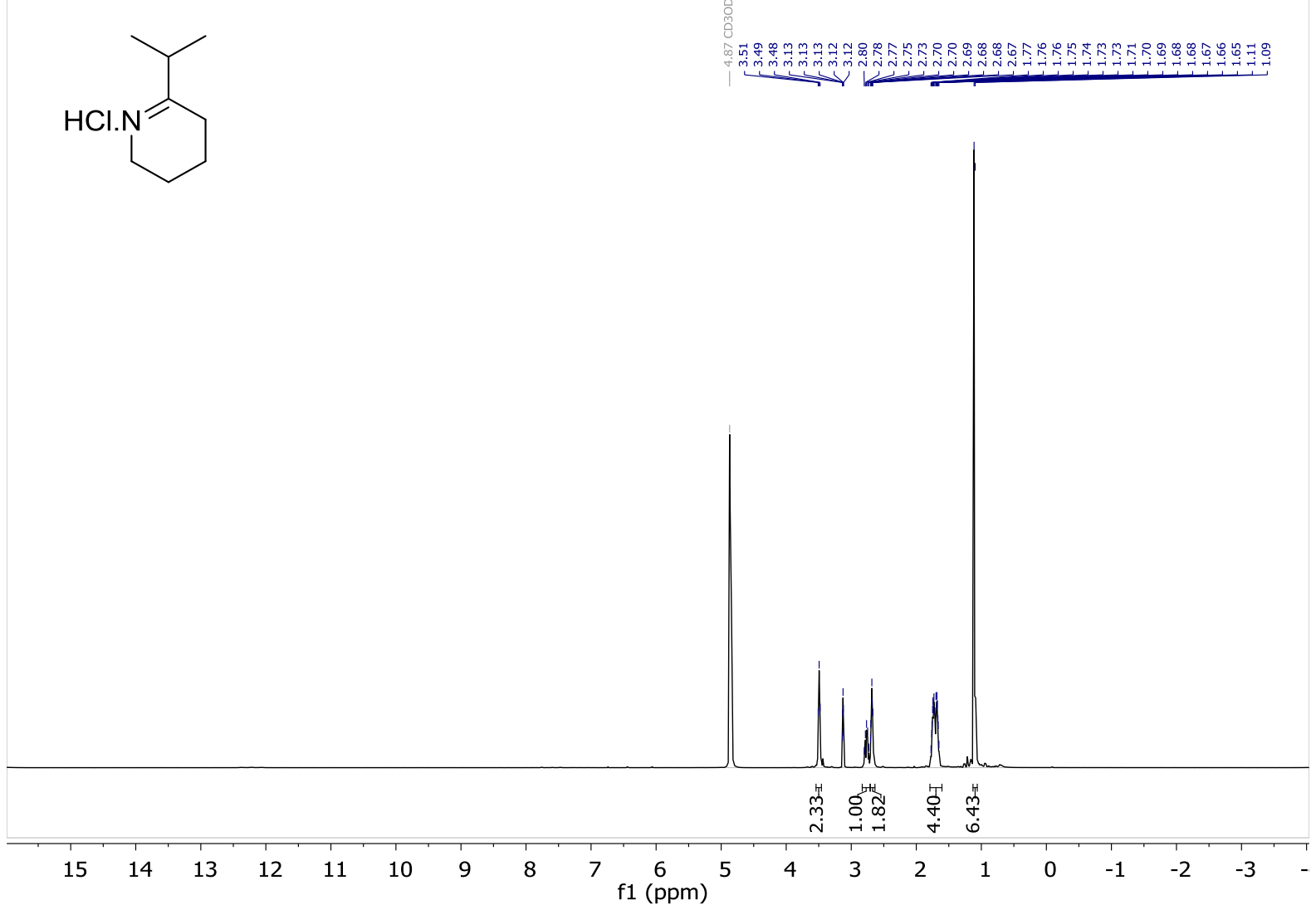

${ }^{13}$ C NMR (100 MHz, CD $\left.3 \mathrm{OD}\right)$

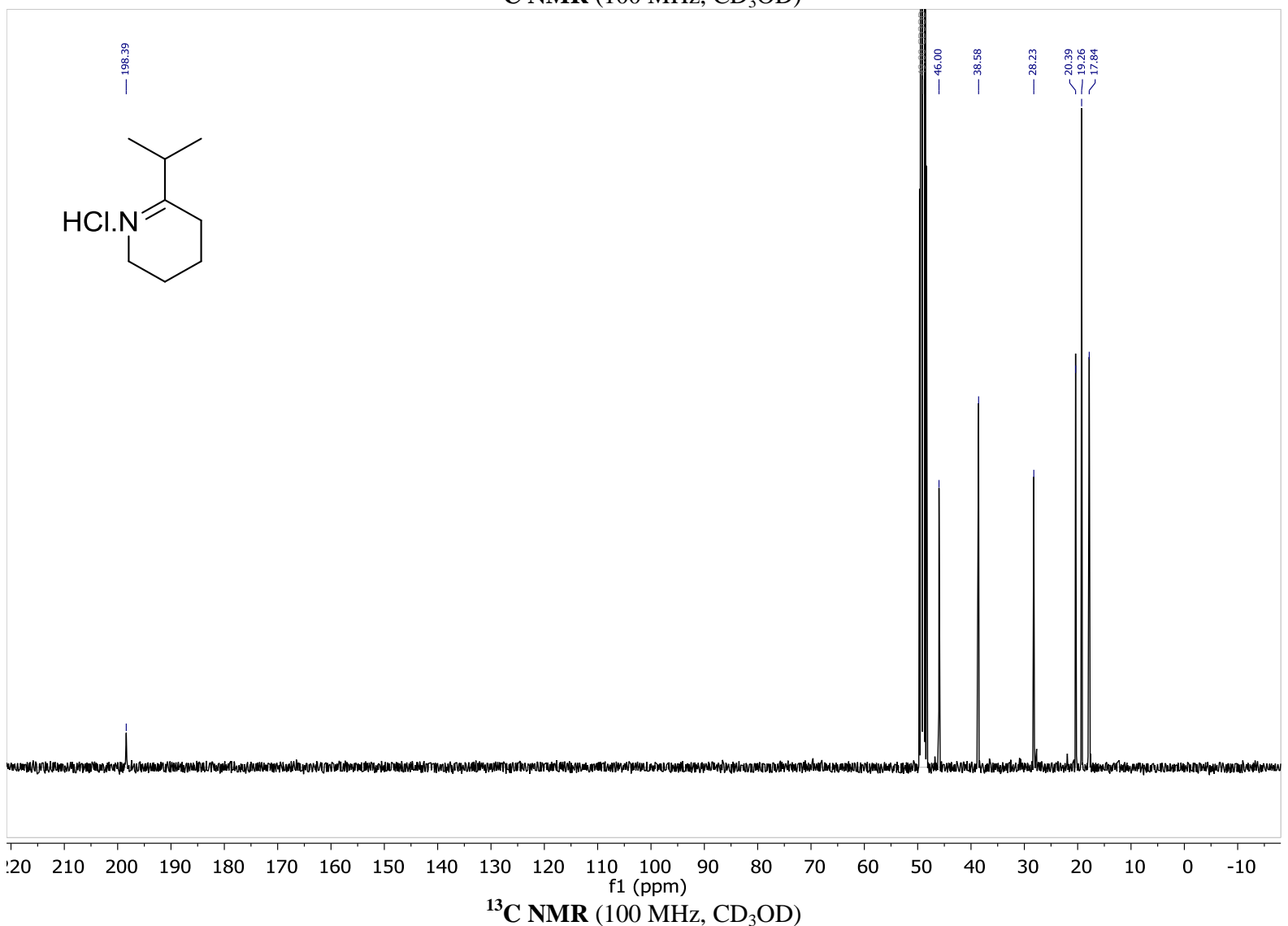


TTOCT18-135

6-(sec-butyl)-2,3,4,5-tetrahydropyridine hydrochloride 14b

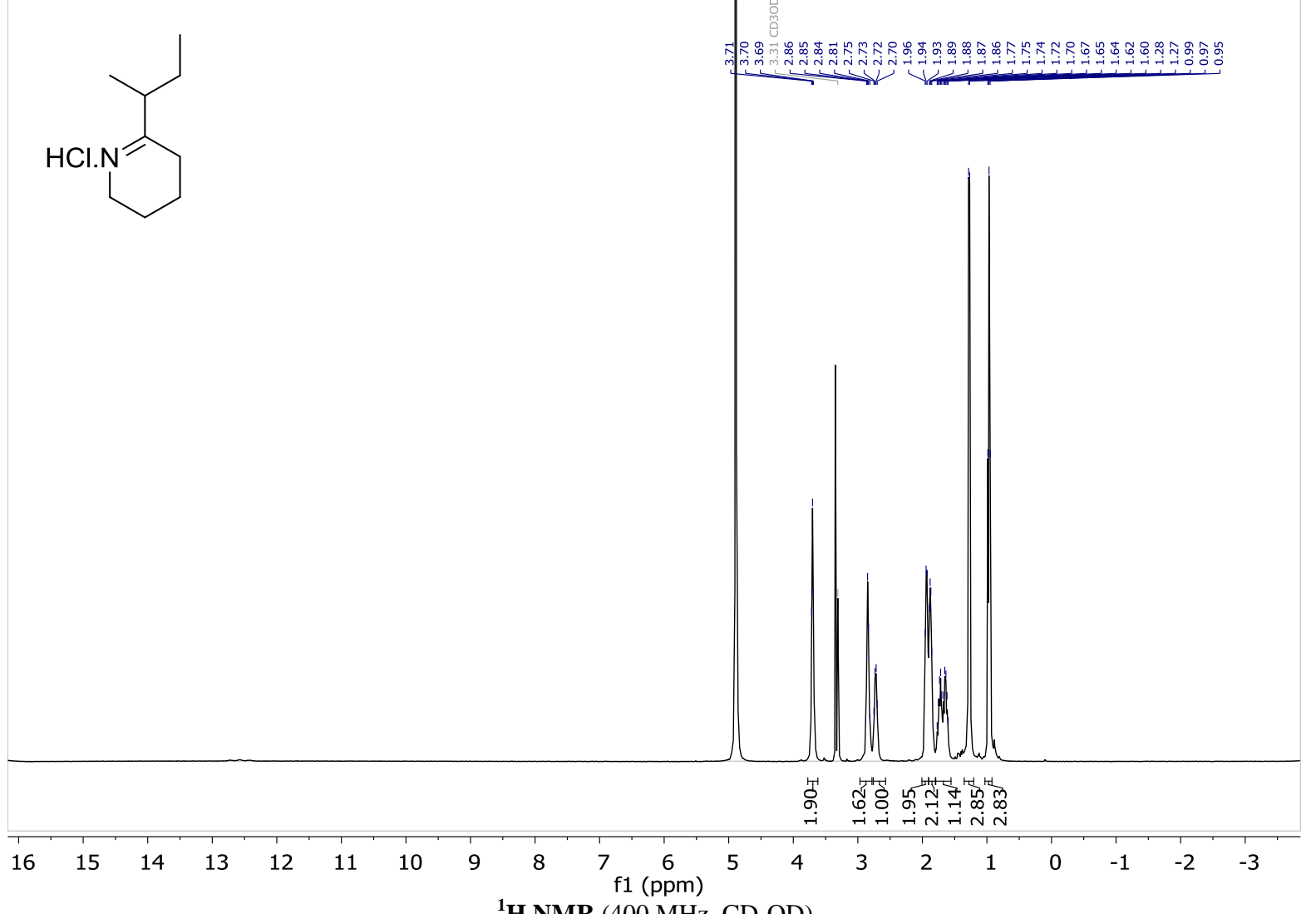

${ }^{1} \mathbf{H}$ NMR $\left(400 \mathrm{MHz}, \mathrm{CD}_{3} \mathrm{OD}\right)$

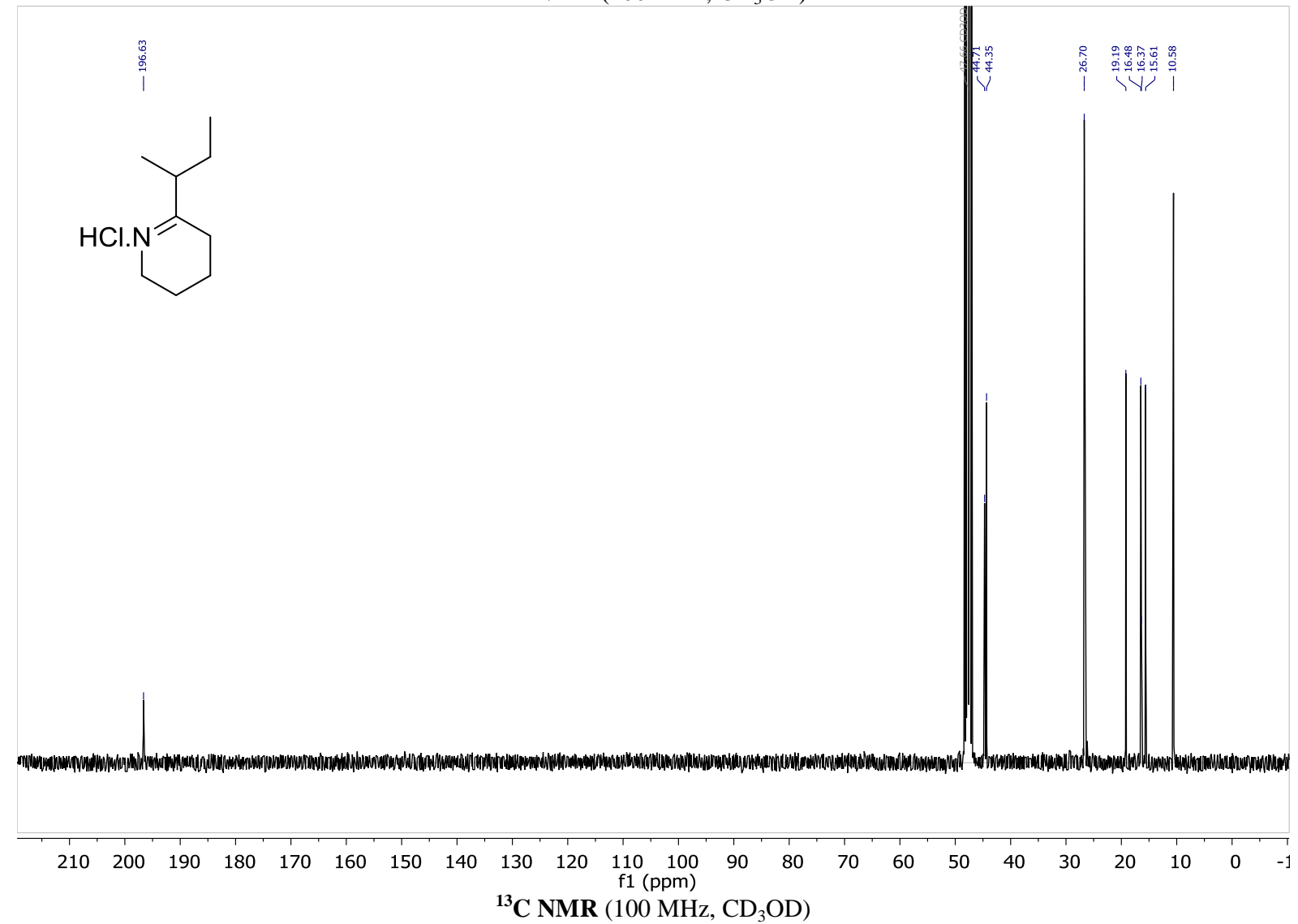


TTAUG18-97

9-amino-3-methylnonan-4-one trifluoroacetate 15b

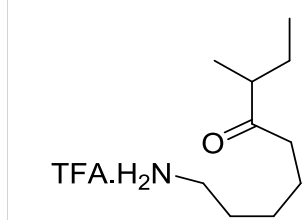

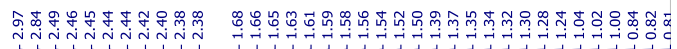

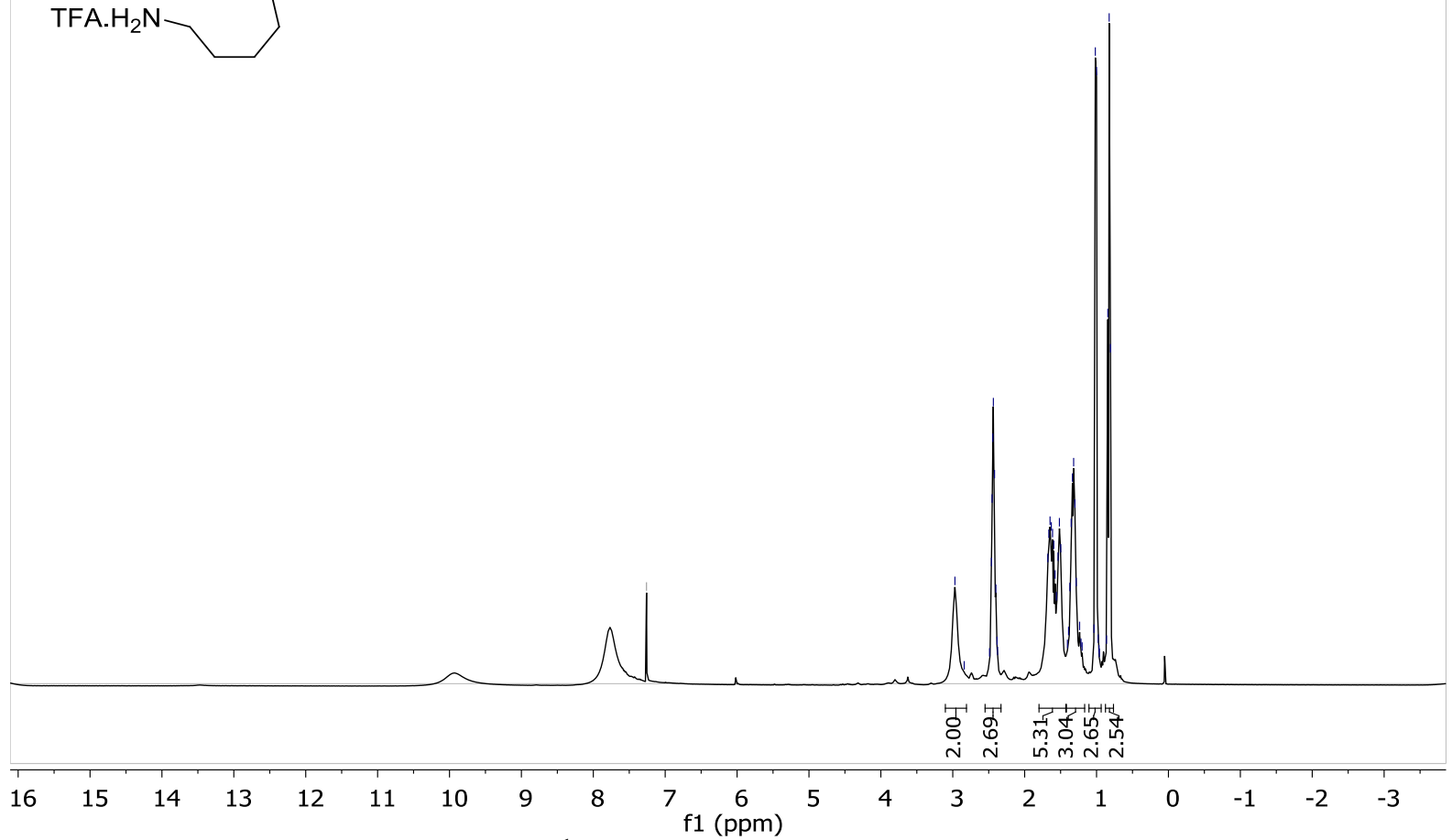

${ }^{1} \mathbf{H}$ NMR $\left(400 \mathrm{MHz}, \mathrm{CDCl}_{3}\right)$

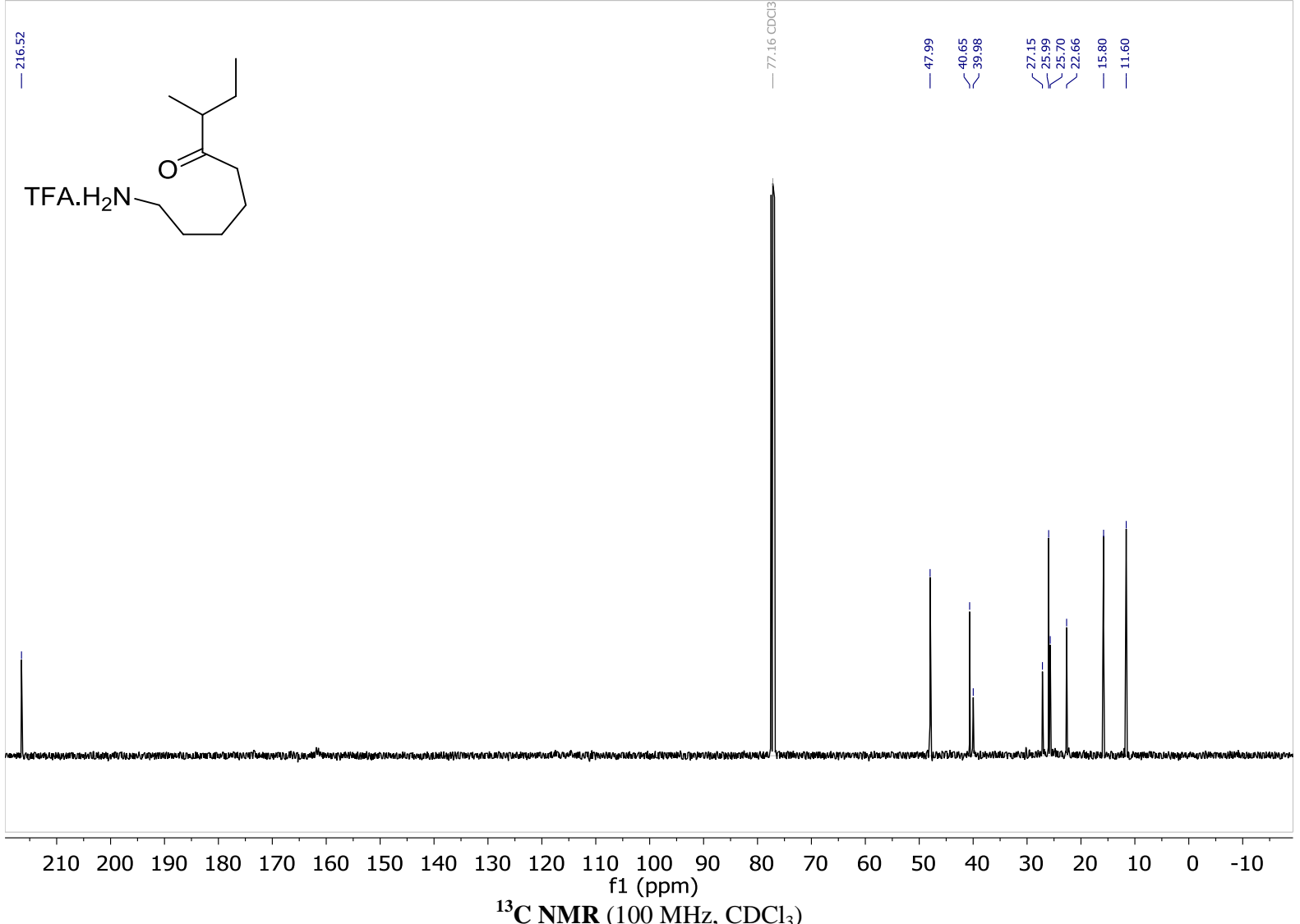


6.1.5 Cyclic amines (standards)

TTAUG18-69

2-(sec-butyl)pyrrolidine 16b

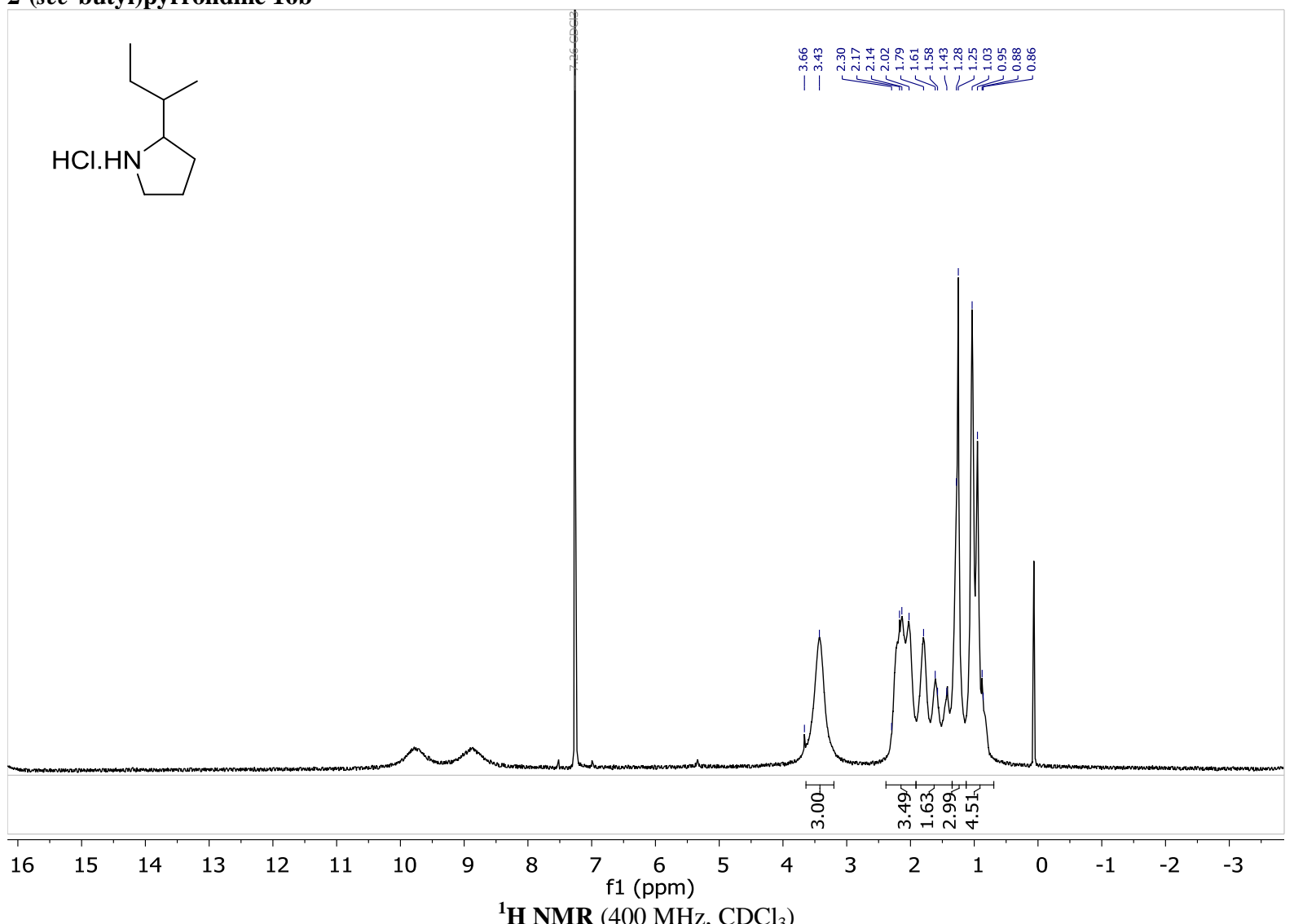


TTAUG18-51

2-(iso-propyl)piperidine 17a
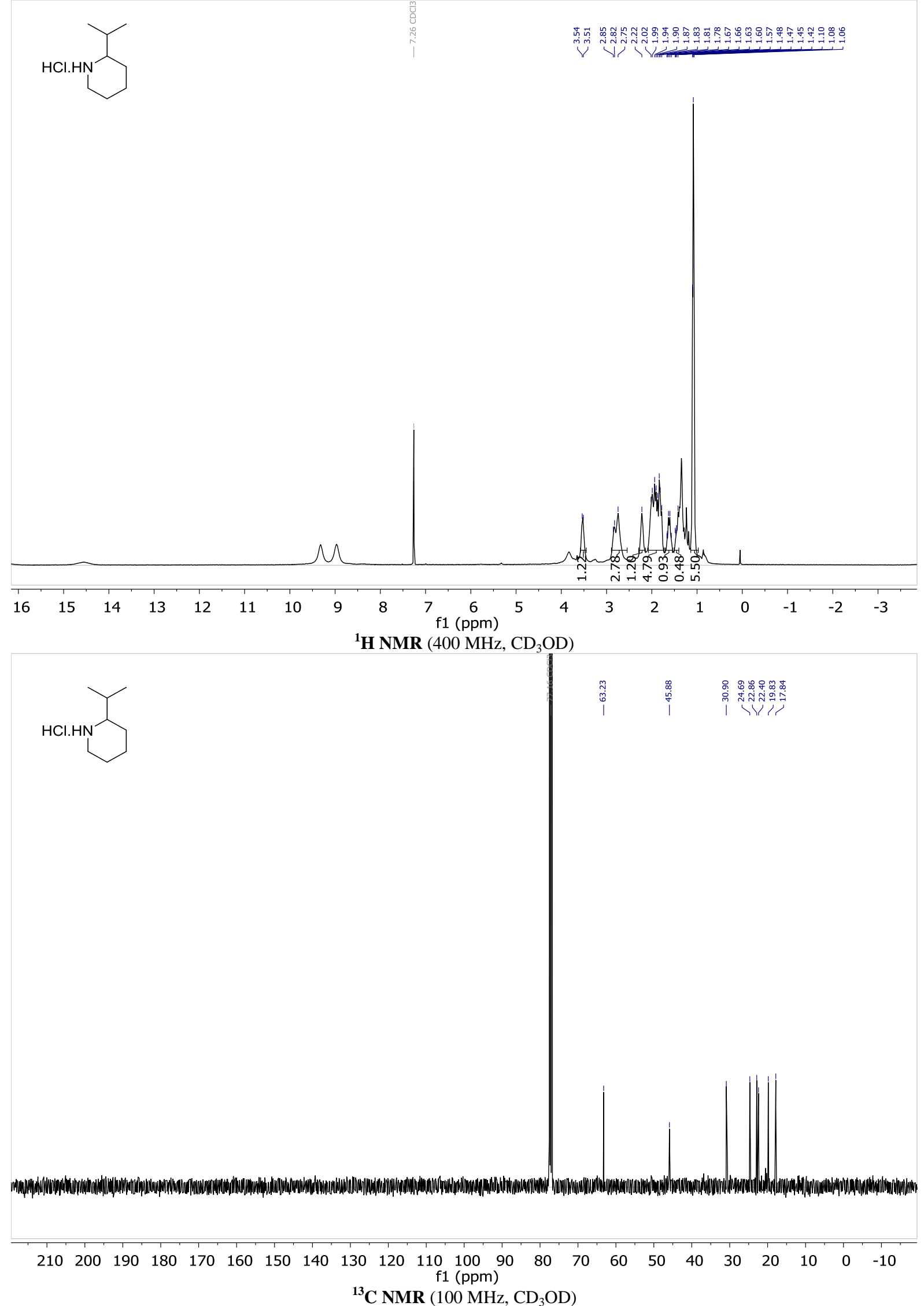
TTOCT18-180

2-(sec-butyl)piperidine 17b
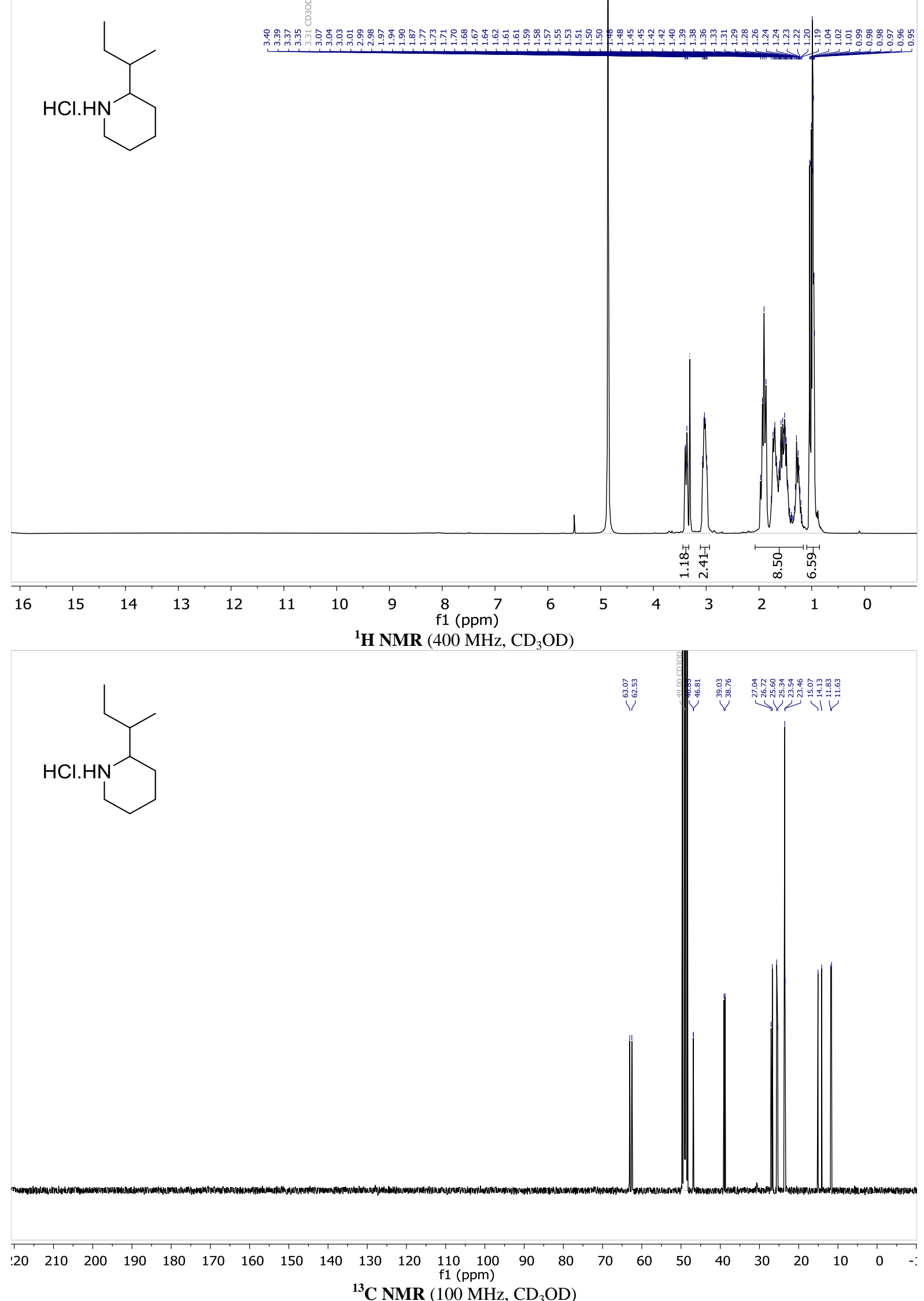
6.1.6 Cyclic amines (biotransformation products)

TTBIO85

(R)-2-(iso-propyl)piperidine $(R)-17$ a

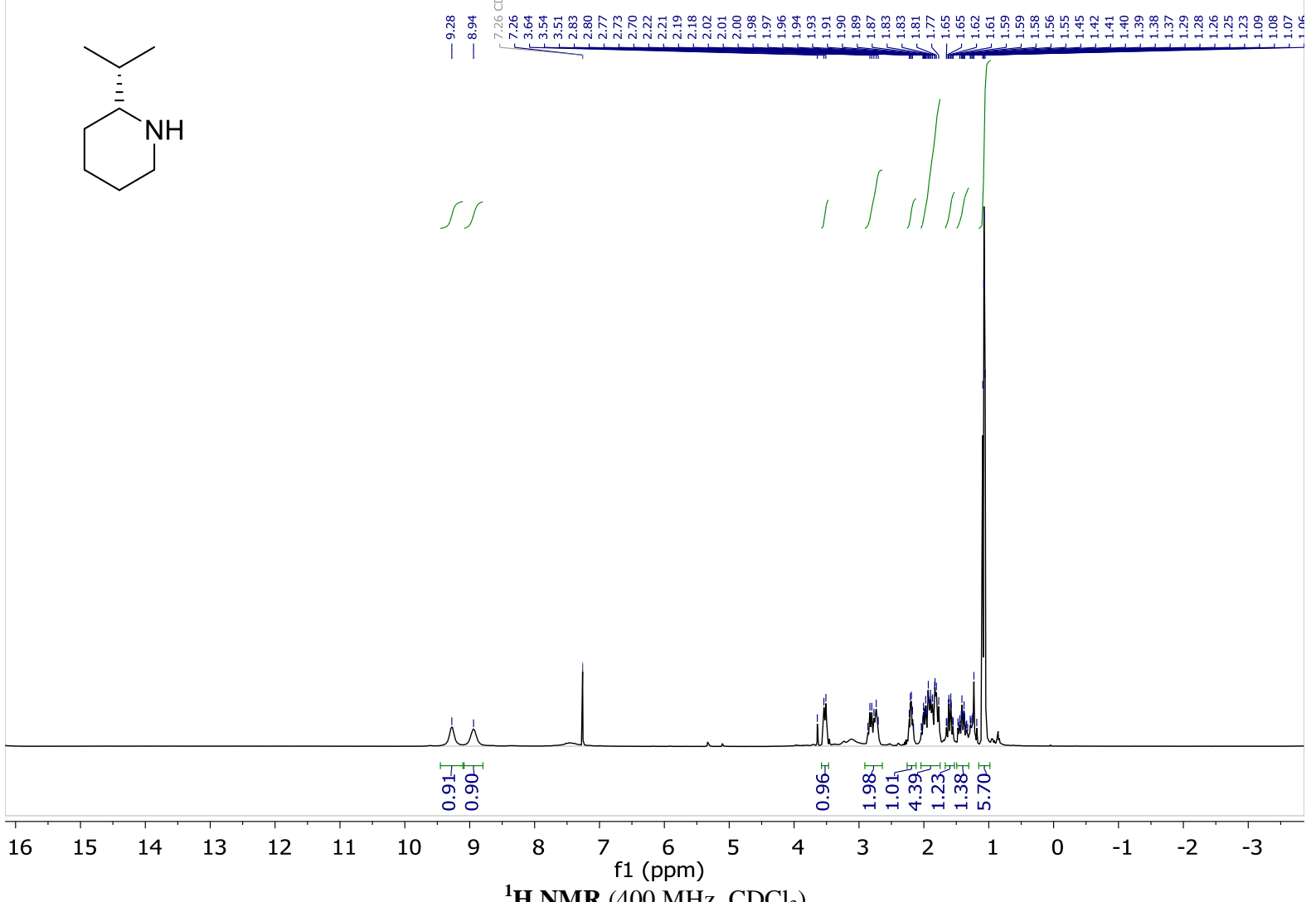

${ }^{1} \mathbf{H}$ NMR $\left(400 \mathrm{MHz}, \mathrm{CDCl}_{3}\right)$

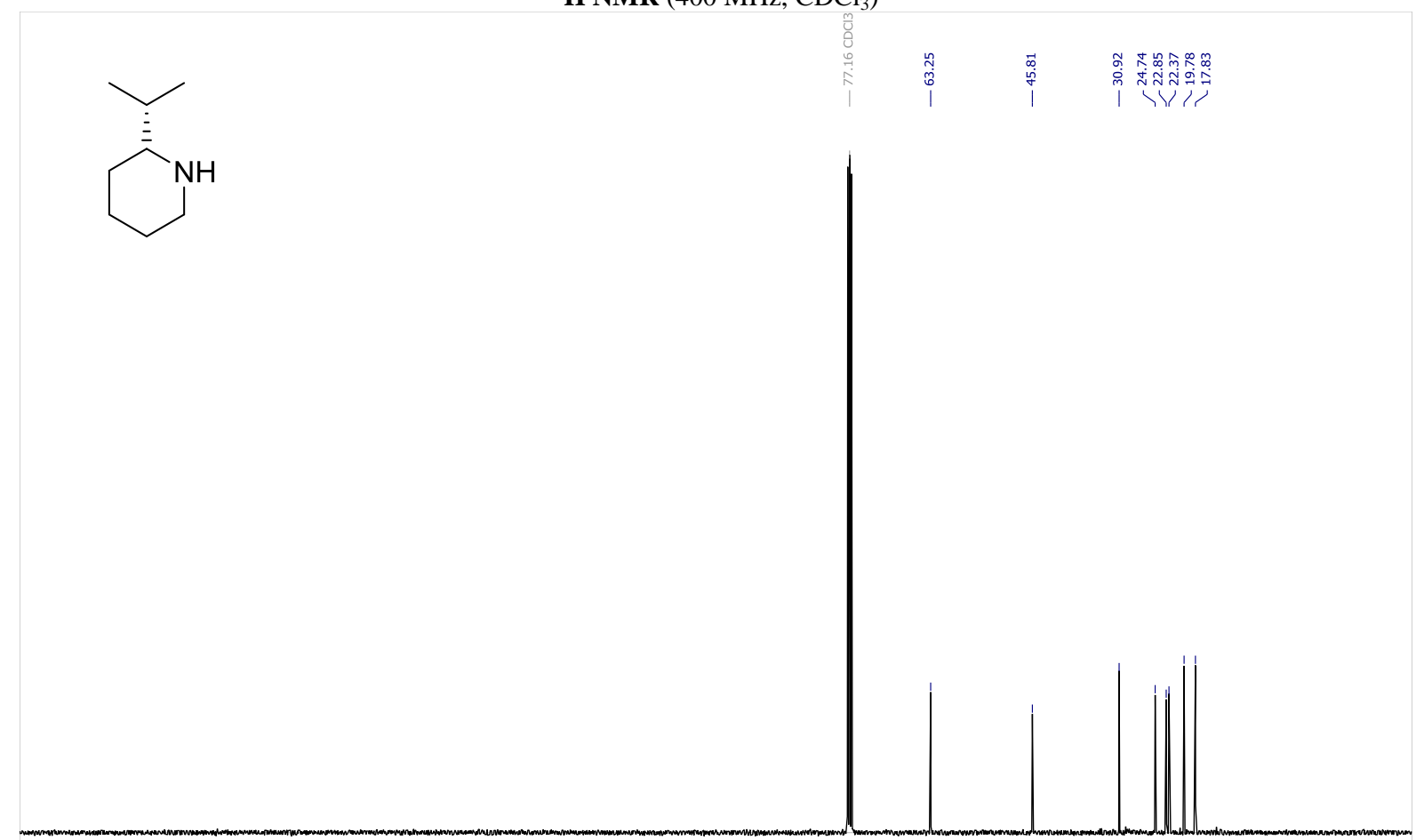

$\begin{array}{lllllllllllllllllllllll}210 & 200 & 190 & 180 & 170 & 160 & 150 & 140 & 130 & 120 & 110 & 100 & 90 & 80 & 70 & 60 & 50 & 40 & 30 & 20 & 10 & 0 & -10\end{array}$ ${ }^{13}$ C NMR (100 MHz, $\left.\mathrm{CDCl}_{3}\right)$ 
TTBIO141

$(R)-2-((S)$-sec-butyl)piperidine $(R S)-17 b$

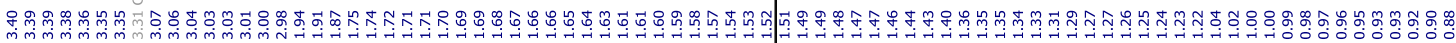<smiles>CC[C@H](C)[C@H]1CCCCN1</smiles>

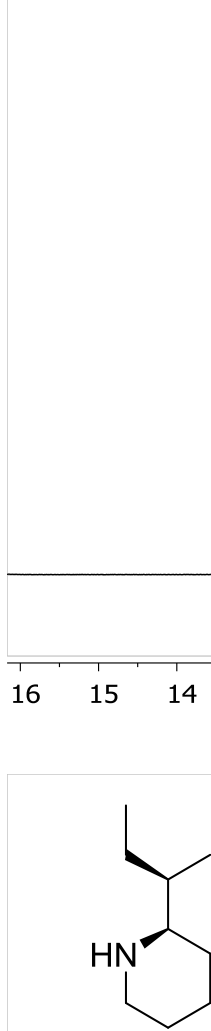

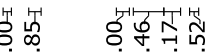

, , ला ता

f1 ( $\mathrm{ppm})$

${ }^{1} \mathbf{H}$ NMR $\left(400 \mathrm{MHz}, \mathrm{CDCl}_{3}\right)$

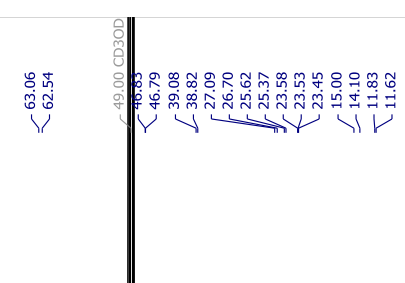

$\begin{array}{lllllllllllllllllllllllll}20 & 210 & 200 & 190 & 180 & 170 & 160 & 150 & 140 & 130 & 120 & 110 & 100 & 90 & 80 & 70 & 60 & 50 & 40 & 30 & 20 & 10 & 0 & -10\end{array}$ ${ }^{13} \mathbf{C}$ NMR $\left(100 \mathrm{MHz}, \mathrm{CDCl}_{3}\right)$ 


\subsection{NMR studies}

A ${ }^{1} \mathrm{H}$ NMR study was undertaken to understand the rate of imine-enamine tautomerisation in the biotransformations by mimicking the reaction conditions. 13a was dissolved in $\mathrm{D}_{2} \mathrm{O}: 1 \mathrm{M} \mathrm{pH} 7.0$ phosphate buffer (90:10) and monitored periodically over $50 \mathrm{~h}$. Over $24 \mathrm{~h}$ an average of two $\alpha$-imine protons had exchanged for deuterium after $24 \mathrm{~h}$ demonstrating that racemisation of $\alpha$-chiral imines can occur during the biotransformations. However this exchange is too slow for an effective enzymatically controlled DKR process.

Figure S1. ${ }^{1} \mathrm{H}$ NMR Study of imine-enamine tautomerisation of cyclic imine 13a.<smiles>[2H]C(C)(C)C1=CCCN1</smiles><smiles>CC1N=C(C(C)(C)C)C(C)(C)C1[18OH]</smiles>

$13 a$<smiles>CC(C)C1=CCCN1</smiles>
$\mathrm{D}_{2} \mathrm{O}$<smiles>[2H]C1CCN=C1C(C)C</smiles>

\section{$\mathrm{H}_{\mathrm{B}}+\mathrm{H}_{\mathrm{C}}$}
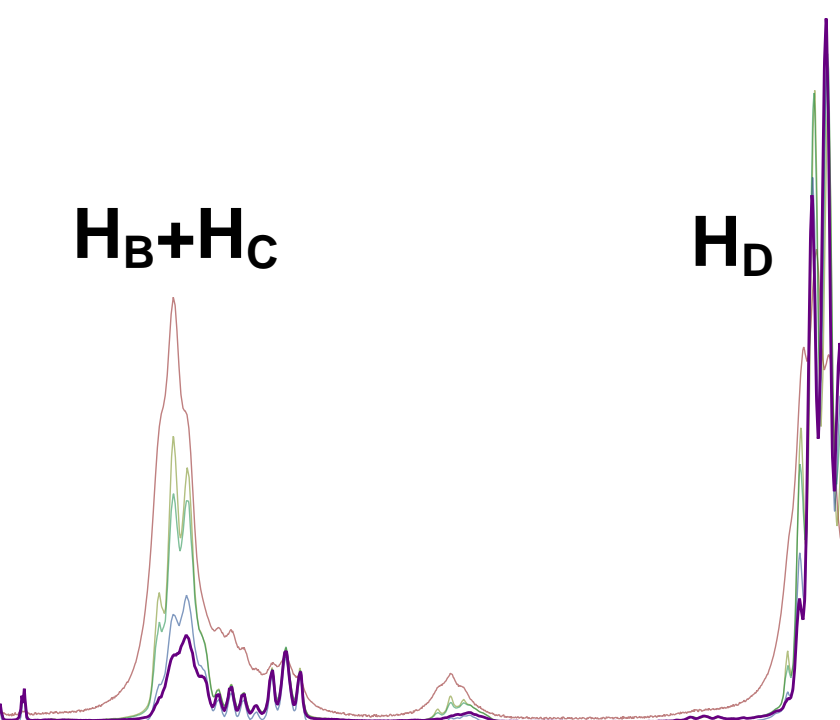

\begin{tabular}{lllllllllllllllllllllll}
\hline 4.2 & 4.1 & 4.0 & 3.9 & 3.8 & 3.7 & 3.6 & 3.5 & 3.4 & 3.3 & $\begin{array}{c}3.2 \\
\mathrm{f} 1(\mathrm{ppm})\end{array}$ & 3.1 & 3.0 & 2.9 & 2.8 & 2.7 & 2.6 & 2.5 & 2.4 & 2.3 & 2.2 & 2.1
\end{tabular}

\begin{tabular}{|c|c|c|c|}
\hline Spectrum & $\begin{array}{l}\text { Recording time } \\
{[\mathrm{h}]}\end{array}$ & $\begin{array}{c}\text { Ratio } \\
\mathrm{A}: \mathrm{B}+\mathrm{C}\end{array}$ & $\begin{array}{c}\text { Ratio } \\
\text { B+C : D }\end{array}$ \\
\hline Methanol- $d_{4}$ & n.a. & $2.0: 3.3$ & $2.9: 2.0$ \\
\hline 1 & 16 & $2.0: 1.2$ & $1.2: 2.0$ \\
\hline 2 & 24 & $2.0: 1.0$ & $1.0: 2.0$ \\
\hline 3 & 40 & $2.0: 0.6$ & $0.6: 2.0$ \\
\hline 4 & 64 & $2.0: 0.6$ & $0.5: 2.0$ \\
\hline
\end{tabular}


A ${ }^{1} \mathrm{H}$ NMR study was undertaken to understand the cyclic enimine-amino enone equilibrium position within the biotransformations by mimicking the reaction conditions. 5d or 25a was dissolved in $\mathrm{D}_{2} \mathrm{O}: 100 \mathrm{mM}$ pH 7.0 phosphate buffer (10:90) and analysed immediately by ${ }^{1} \mathrm{H}$ NMR.

Figure S2. ${ }^{1} \mathrm{H}$ NMR Study of cyclic enimine-amino enone equilibrium 13a.<smiles></smiles>

5d

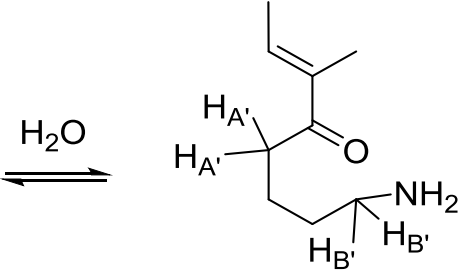

24d

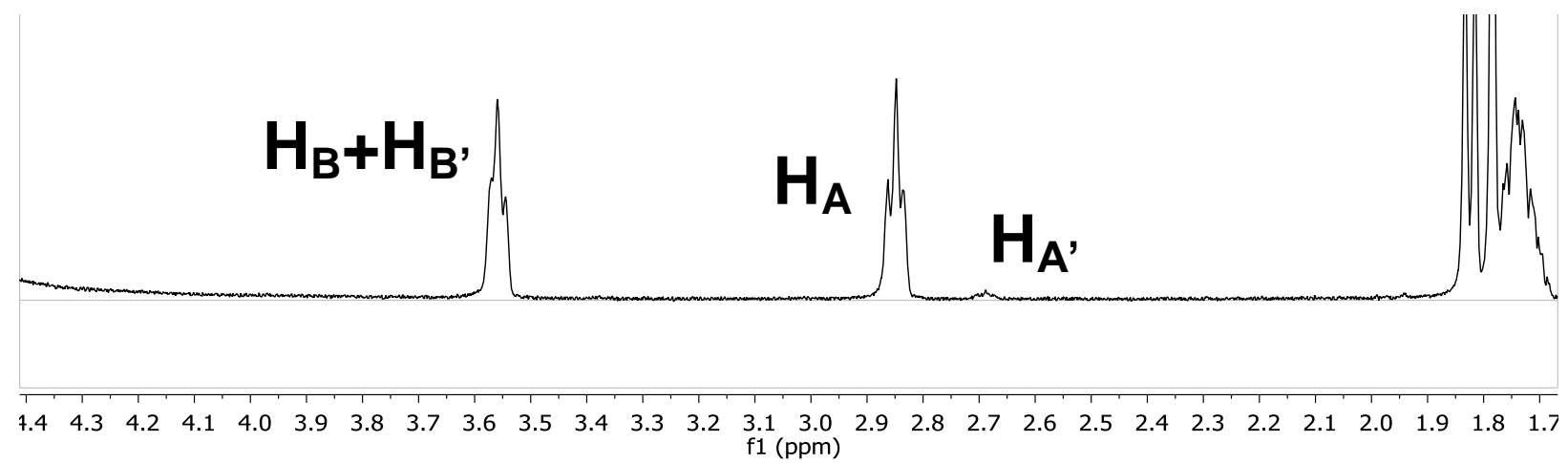<smiles></smiles>

$\mathrm{H}_{2} \mathrm{O}$
$\mathrm{H}_{B}$

$6 a$<smiles></smiles>

$25 a$

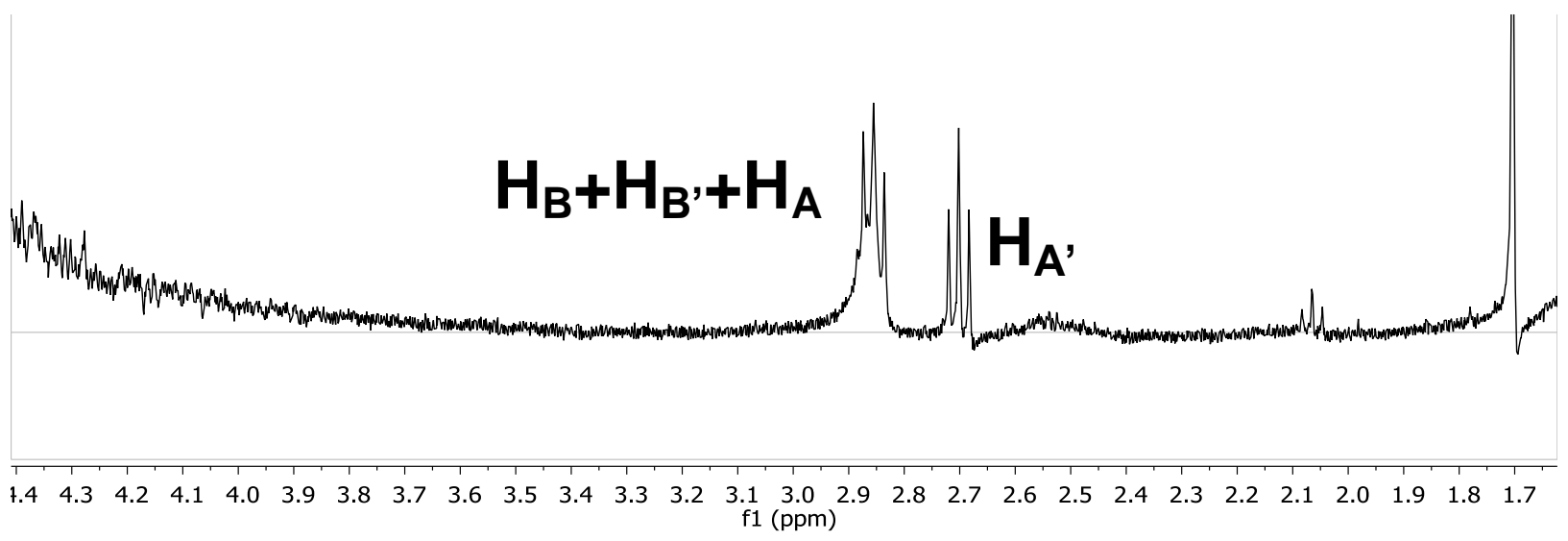


Figure S3. Overlay of the ${ }^{1} \mathrm{H}$ NMR spectra of enriched $(R S)$-17b obtained from biotransformation vs. rac-17b.

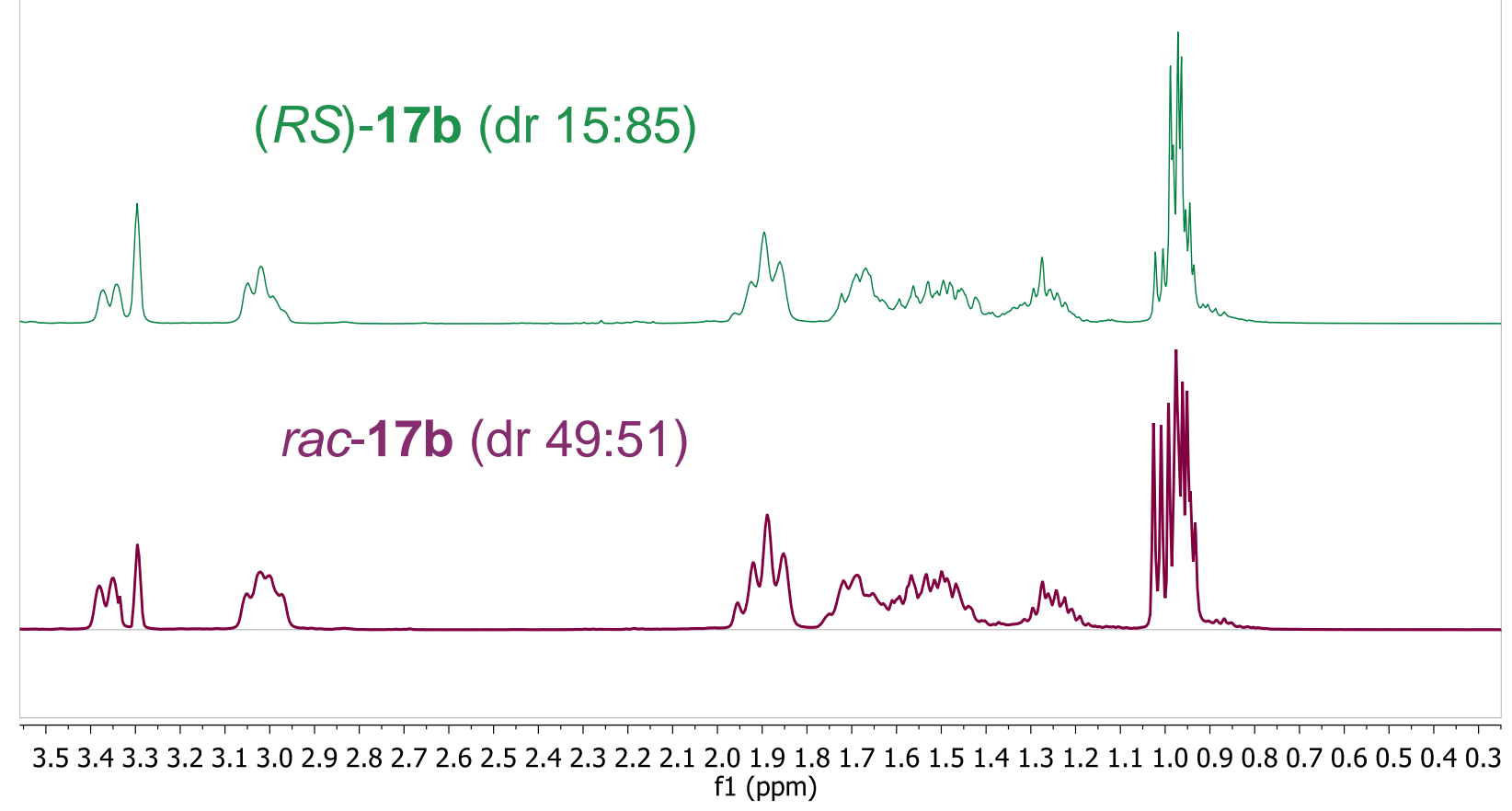


(S1) Bechtold, M.; Brenna, E.; Femmer, C.; Gatti, F. G.; Panke, S.; Parmeggiani, F.; Sacchetti, A. Biotechnological Development of a Practical Synthesis of Ethyl (S)-2-Ethoxy-3-(p-Methoxyphenyl)Propanoate (EEHP): Over 100Fold Productivity Increase from Yeast Whole Cells to Recombinant Isolated Enzymes. Org. Process Res. Dev. 2012, 16 (2), 269-276.

(S2) Adalbjörnsson, B. V.; Toogood, H. S.; Fryszkowska, A.; Pudney, C. R.; Jowitt, T. A.; Leys, D.; Scrutton, N. S. Biocatalysis with Thermostable Enzymes: Structure and Properties of a Thermophilic 'Ene'-Reductase Related to Old Yellow Enzyme. ChemBioChem 2010, 11 (2), 197-207.

(S3) Mansell, D. J.; Toogood, H. S.; Waller, J.; Hughes, J. M. X.; Levy, C. W.; Gardiner, J. M.; Scrutton, N. S. Biocatalytic Asymmetric Alkene Reduction: Crystal Structure and Characterization of a Double Bond Reductase from Nicotiana tabacum. ACS Catal. 2013, 3 (3), 370-379.

(S4) Hulley, M. E.; Toogood, H. S.; Fryszkowska, A.; Mansell, D.; Stephens, G. M.; Gardiner, J. M.; Scrutton, N. S. Focused Directed Evolution of Pentaerythritol Tetranitrate Reductase by Using Automated Anaerobic Kinetic Screening of Site-Saturated Libraries. ChemBioChem 2010, 11 (17), 2433-2447.

(S5) Hussain, S.; Leipold, F.; Man, H.; Wells, E.; France, S. P.; Mulholland, K. R.; Grogan, G.; Turner, N. J. An (R)Imine Reductase Biocatalyst for the Asymmetric Reduction of Cyclic Imines. ChemCatChem 2015, 7 (4), 579-583.

(S6) Leipold, F.; Hussain, S.; Ghislieri, D.; Turner, N. J. Asymmetric Reduction of Cyclic Imines Catalyzed by a WholeCell Biocatalyst Containing an (S)-Imine Reductase. ChemCatChem 2013, 5 (12), 3505-3508.

(S7) Aleku, G. A.; France, S. P.; Man, H.; Mangas-Sanchez, J.; Montgomery, S. L.; Sharma, M.; Leipold, F.; Hussain, S.; Grogan, G.; Turner, N. J. A Reductive Aminase from Aspergillus oryzae. Nat. Chem. 2017, 9 (10), 961-969.

(S8) Giovannini, A.; Savoia, D.; Umani-Ronchi, A. Organometallic Ring-Opening Reactions of $N$-Acyl and $N$ Alkoxycarbonyl Lactams. Synthesis of Cyclic Imines. J. Org. Chem. 1989, 54 (1), 228-234.

(S9) Burke, A. J.; Davies, S. G.; Garner, A. C.; McCarthy, T. D.; Roberts, P. M.; Smith, A. D.; Rodriguez-Solla, H.; Vickers, R. J. Asymmetric Synthesis and Applications of $\beta$-Amino Weinreb Amides: Asymmetric Synthesis of $(S)$ Coniine. Org. Biomol. Chem. 2004, 2 (9), 1387-1394.

(S10) Adriaenssens, L. V.; Austin, C. A.; Gibson, M.; Smith, D.; Hartley, R. C. Stereodivergent Diversity Oriented Synthesis of Piperidine Alkaloids. Eur. J. Org. Chem. 2006, 2006 (22), 4998-5001. 